\title{
Statische Auszugstragfähigkeit gewindeformender Schrauben in Metallkonstruktionen
}

\author{
Zur Erlangung des akademischen Grades eines \\ DOKTOR-INGENIEURS \\ DOKTORS DER NATURWISSENSCHAFTEN \\ von der Fakultät für \\ Bauingenieur-, Geo- und Umweltwissenschaften \\ der Universität Fridericiana zu Karlsruhe $(\mathrm{TH})$ \\ genehmigte \\ DISSERTATION \\ von
}

Dipl.-Ing. Roderich Hettmann

aus Arad (Rumänien)

Tag der mündlichen

Prüfung: 06.12.2006

Hauptreferent: Prof. Dr.-Ing. H. Saal

Korreferent: Prof. Dr.-Ing. T. Seeger

Karlsruhe 2006 


\section{Inhaltsverzeichnis}

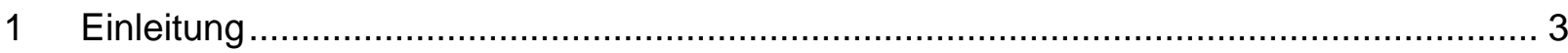

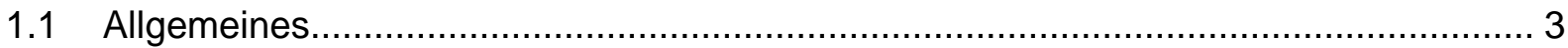

1.2 Problemstellung und Ziel der Arbeit............................................................... 7

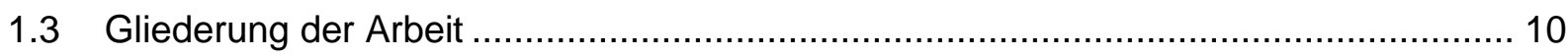

2 Charakteristische Eigenschaften der untersuchten Verbindungen ............................... 12

2.1 Verbindungstypen und Versagensmechanismen................................................. 12

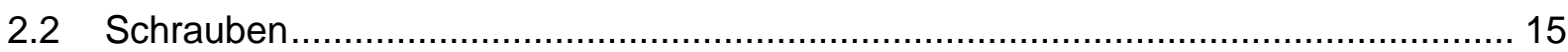

2.2.1 Zusammenhang zwischen Schraubengeometrie, Montagevorgang und Anwendungsbereich ...................................................................................... 15

2.2.2 Ausgangswerkstoffe und mechanische Eigenschaften..................................... 28

2.3 Versuche zur Bestimmung der Auszugstragfähigkeit............................................. 34

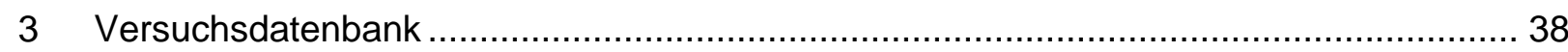

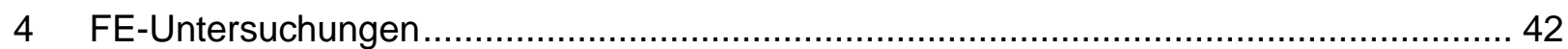

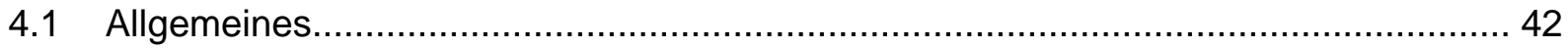

4.2 Simulierte Versuchsserien der Datenbank ................................................................ 42

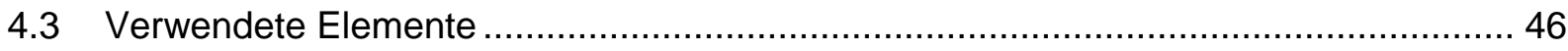

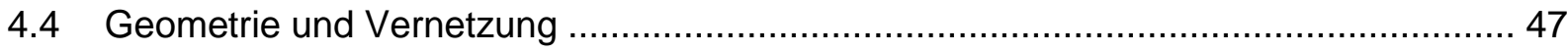

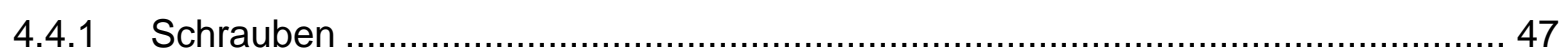

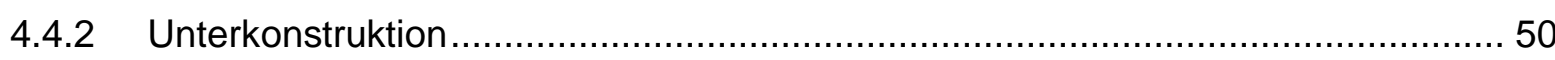

4.5 Berechnungsansätze zur Beschreibung des Werkstoffverhaltens der

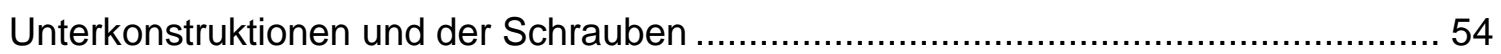

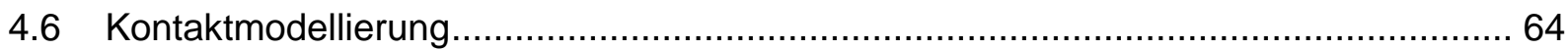

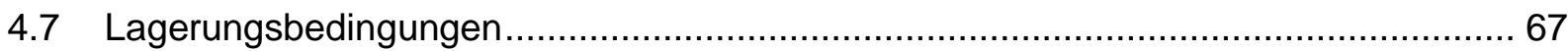

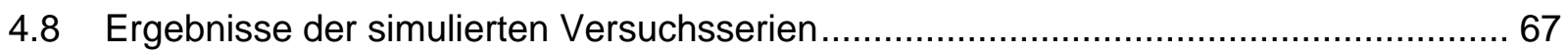

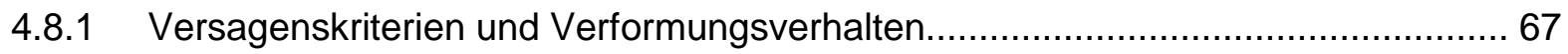

4.8.2 Vergleich der FE-Ergebnisse mit den Versuchsergebnissen ................................. 76

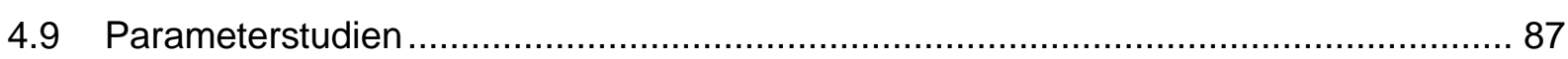

4.9.1 Bandbreite der Parameterstudien............................................................ 87

4.9.2 Entwicklung eines mechanischen Modells und formelmäßige Beschreibung der Auszugstragfähigkeit anhand der Ergebnisse der Parameterstudien .................... 90

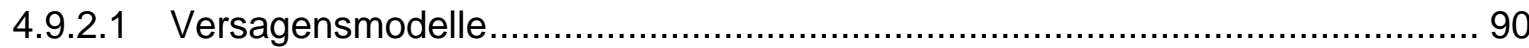

4.9.2.2 Kalibrierung des mechanischen Modells anhand der Ergebnisse der

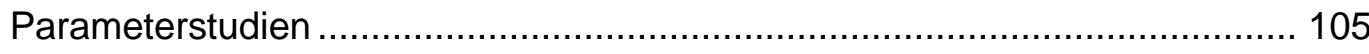




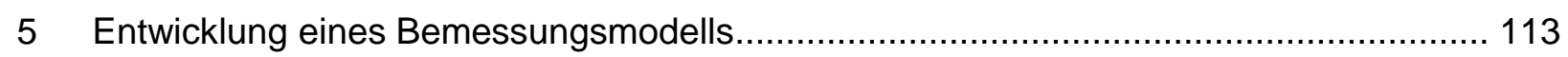

5.1 Anwendung des mechanischen Modells auf die Versuchsergebnisse ....................... 113

5.2 Charakteristischer Wert der Beanspruchbarkeit................................................. 119

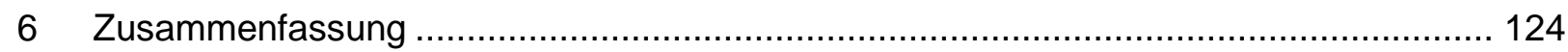

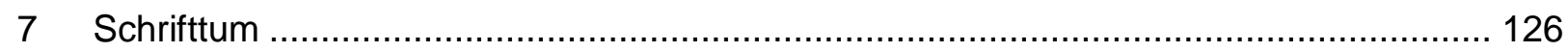

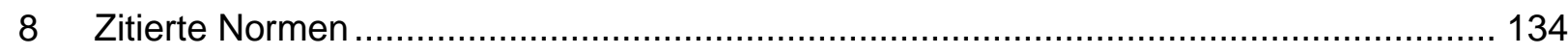

Anhang A: Versuchsdatenbank

Anhang B: Werkstoffgesetze für die FE-Berechnungen

Anhang C: Vergleich der Last-Verformungskurven Versuch/FEM

Anhang D: Ergebnisse der Parameterstudien 


\section{$1 \quad$ Einleitung}

\section{$1.1 \quad$ Allgemeines}

Im Metalleichtbau werden dünnwandige, großflächige Profilbleche (Trapezprofile, Wellprofile, Stehfalzprofile und Kassettenprofile, Bild 1.1) sowie die mit Profilblechen hergestellten Sandwichelemente (Bild 1.2) als raumabschließende Wetterschale und als Tragschale verwendet.

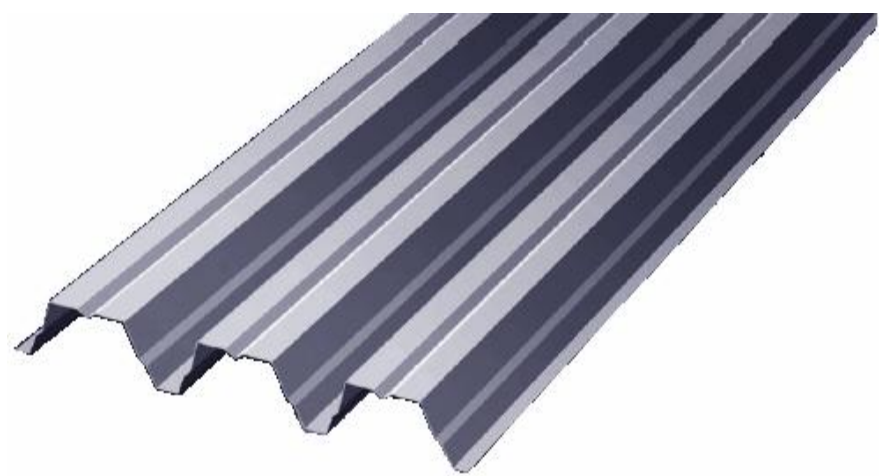

a) Trapezprofil

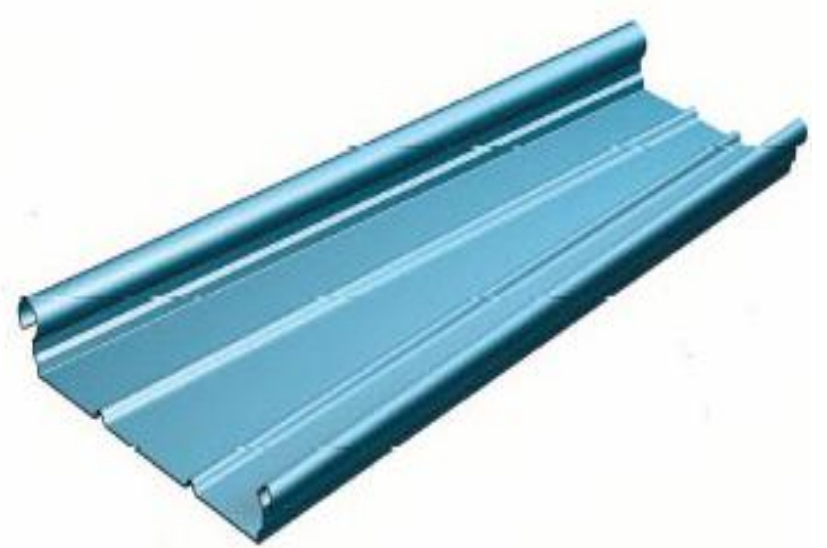

c) Stehfalzprofil

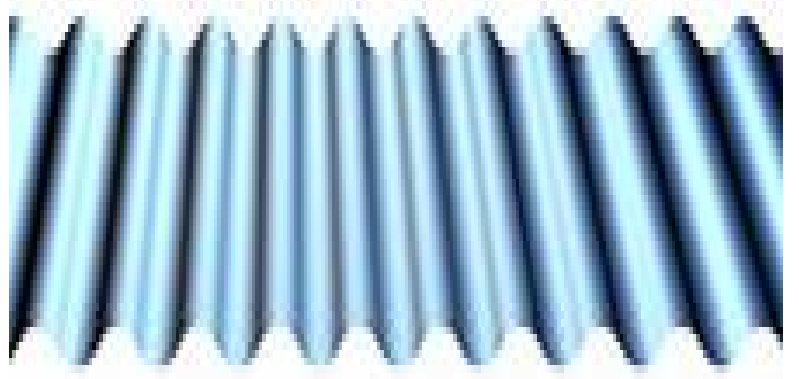

b) Wellprofil

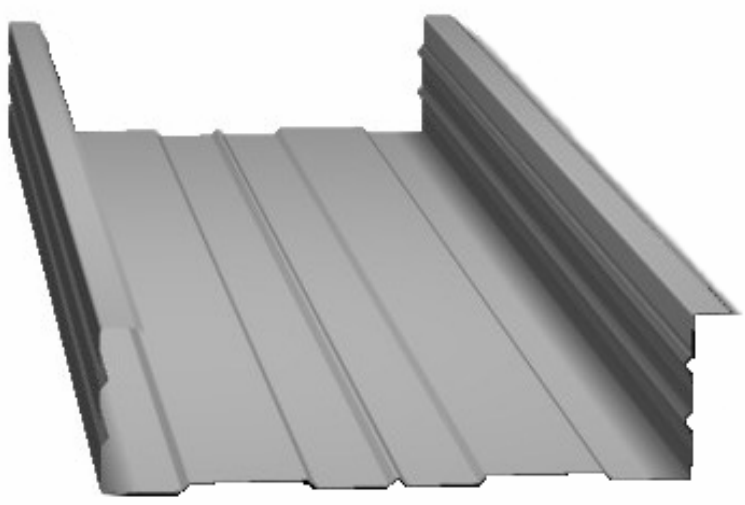

d) Kassettenprofil

Bild 1.1: Profilbleche des Metalleichtbaus

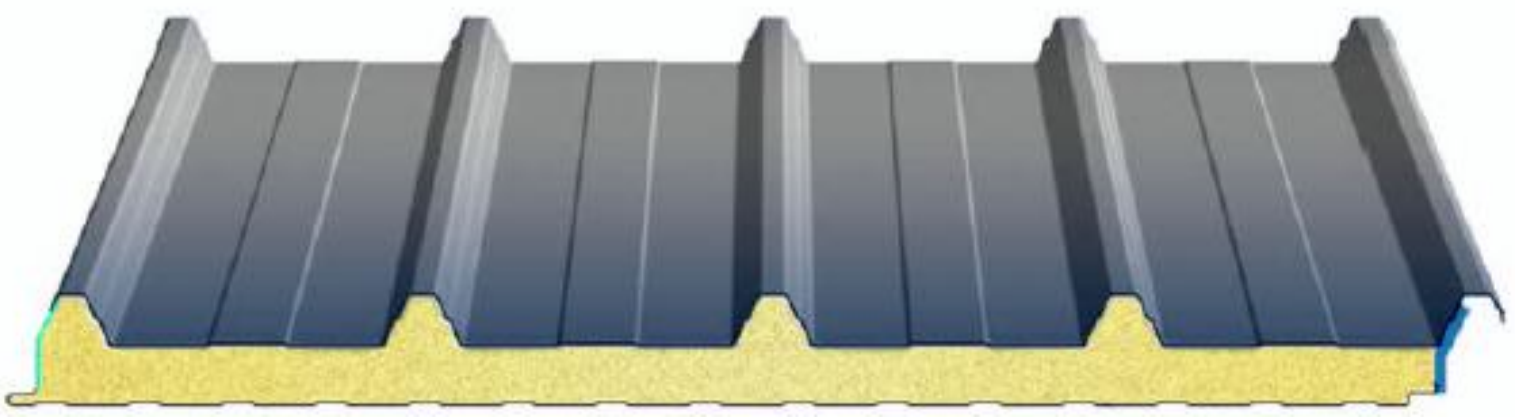

Bild 1.2: Sandwichelement mit profilierten Deckblechen 
Die Formgebung der Profilbleche erfolgt durch die Rollformtechnik, welche die Entwicklung der industriellen - und damit auch der wirtschaftlichen - Großfertigung dieser Bauelemente ermöglichte. Weitere Sachverhalte, die ein wirtschaftliches Bauen mit Profilblechen ermöglichen, sind nach [1] ihre große Tragfähigkeit bei geringem Eigengewicht, geringe Montagekosten sowie die Möglichkeit der funktionalen Kopplung von Raumabschluß und Gestaltungselement für Fassaden auf der einen Seite und Tragstrukturelement auf der anderen Seite.

Wegen der Wirtschaftlichkeit herrscht die Leichtbauweise mit Profilblechen im Industrie- und Gewerbehallenbau vor. Darüber hinaus wird diese Bauweise wegen der Möglichkeit, auch architektonisch anspruchsvolle Fassaden zu realisieren (Bild 1.3), zunehmend im Sporthallen-, Verwaltungs- und Wohnungsbau angewendet.

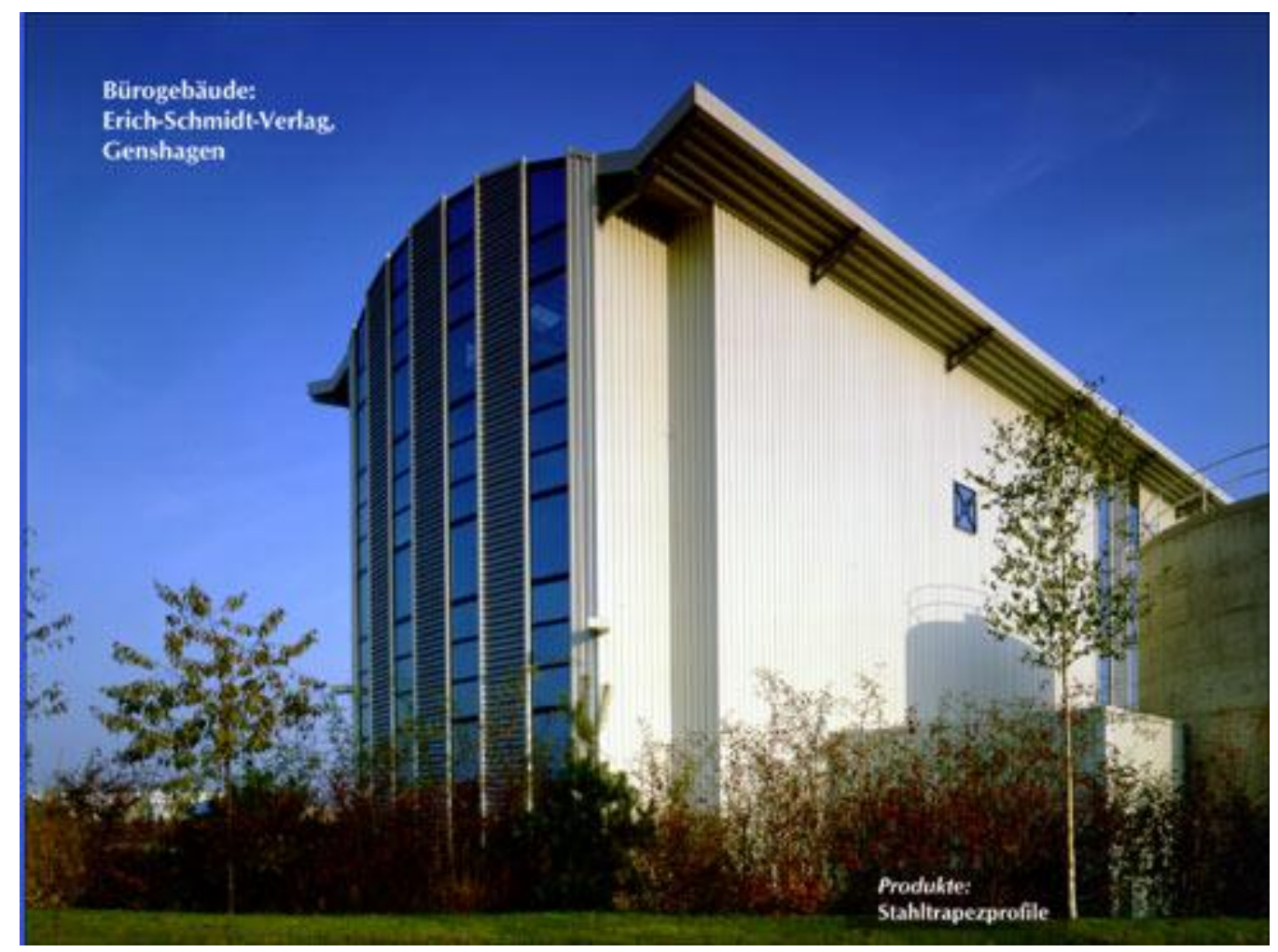

Bild 1.3: Bürogebäude des Erich-Schmidt-Verlags in Genshagen, Fassadenkonstruktion mit Stahltrapezprofilen

Die Verbindung der Profilbleche oder der Sandwichelemente untereinander sowie mit ihrer Unterkonstruktion muß auf die speziellen Anforderungen der Leichtbauweise abgestimmt sein. Zum einen müssen die auftretenden Kräfte sicher aufgenommen und in die teilweise nur wenige Millimeter oder 10tel Millimeter dicke Unterkonstruktion weitergeleitet werden. Zum anderen soll die Verbindung auch abdichtende Funktionen erfüllen und wirtschaftlich herstellbar sein. Dabei kommt der Möglichkeit der Herstellung der Verbindung von einer Seite der Bauelemente eine große Bedeutung zu, da diese Bauelemente in der Regel großflächig sind. 
Für die Leichtbauweise stehen als Verbindungstechniken das Widerstandspreßschweißen (Punktschweißen und Rollnahtschweißen), die Durchsetzfügetechnik (Clinchen) sowie das Verbinden mit Hilfe von mechanischen Verbindungselementen zur Verfügung.

Der Einsatzbereich für das Widerstandspreßschweißen und die Durchsetzfügetechnik ist beschränkt auf die Verbindung benachbarter Profilbleche an ihren Längs- und Querrändern. Für die Verbindung der Profilbleche mit der lastabtragenden Metallunterkonstruktion kommen diese Techniken nicht zum Einsatz. Ein weiterer Nachteil dieser Fügetechniken besteht darin, daß die mit diesen Techniken ausgeführten Verbindungen ohne eine Beschädigung der verbundenen Bauteile nicht mehr lösbar sind.

Als mechanische Verbindungselemente, die im Metalleichtbau zum Einsatz kommen, sind gewindeformende Schrauben (Bohrschrauben und gewindefurchende Schrauben), Blindniete und Setzbolzen zu nennen (Bild 1.4). Alle diese Verbindungselemente erfüllen die Anforderung der einseitigen Montierbarkeit.

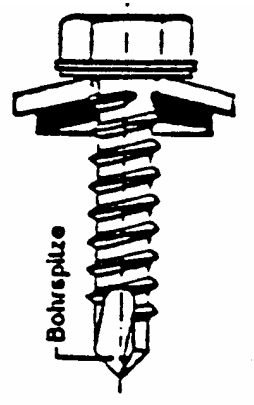

a) Bohrschraube

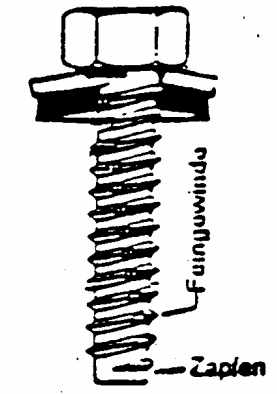

b) Gewindefurchende Schraube Typ B

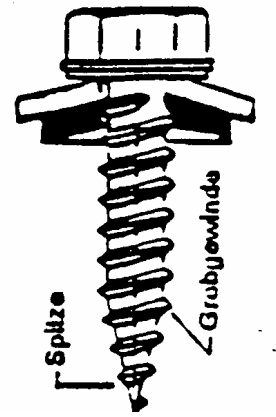

c) Gewindefurchende Schraube Typ A

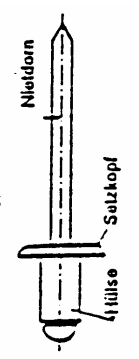

d) Blindniet

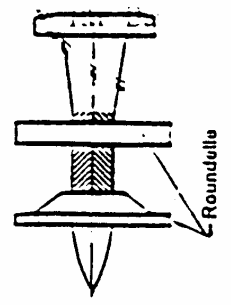

e) Setzbolzen

Bild 1.4: Mechanische Verbindungselemente im Metalleichtbau

Blindniete werden aufgrund ihrer relativ geringen Scher- und Zugbruchtragfähigkeit und dem dadurch bedingten geringen Verformungsvermögen der mit ihnen hergestellten Verbindungen hauptsächlich für die Verbindung benachbarter Profilbleche an ihren Längsrändern (Längsstoß) verwendet.

Der Einsatzbereich von Setzbolzen ist wegen der Verbindungstechnik beschränkt auf die Verbindung von Stahlblechprofilen mit Stahlunterkonstruktionen, deren Dicke nicht kleiner ist als $6 \mathrm{~mm}$ [2].

Gewindeformende Schrauben sind diejenigen Verbindungselemente, denen die größte Bedeutung für die Leichtbauweise zukommt, da sie die oben erwähnten Anforderungen am besten erfüllen.

Für Sandwichelemente, deren Querschnittshöhe aufgrund des zwischen den Deckblechen angeordneten Dämmaterials bis zu $140 \mathrm{~mm}$ groß werden kann, bieten gewindeformende 
Schrauben mit möglichen Schaftlängen bis zu 160mm, die einzige praktikable Möglichkeit einer Verbindung mit der Unterkonstruktion. Dementsprechend sind in der allgemeinen bauaufsichtlichen Zulassung Nr. Z-14.4-407 [3] für die Verbindungen von Sandwichelementen mit Deckschichten aus Stahl auf ihren Unterkonstruktionen ausschließlich gewindeformende Schrauben aufgeführt.

Die marktführende Stellung der gewindeformenden Schrauben wird auch durch einen Blick in die allgemeine bauaufsichtliche Zulassung Nr. Z-14.1-4 [4] deutlich. Von den dort zusammengestellten insgesamt etwa 250 zugelassenen Verbindungselementen für die Verwendung in Konstruktionen mit Kaltprofilen aus Stahlblech sind fast 90\% gewindeformende Schrauben.

Die gewindeformenden Schrauben erzeugen durch eine Stauchung und Verdrängung des Materials im lastabtragenden Bauteil ihr Muttergewinde spanlos. Während gewindefurchende Schrauben eine Vorbohrung in den zu verbindenden Bauteilen benötigen, übernehmen Bohrschrauben mit Hilfe ihrer Bohrspitze den Bohrvorgang selbst und sind somit in nur einem Arbeitsschritt montierbar. Aufgrund des spanlosen Formens ihres Muttergewindes besitzen gewindeformende Schrauben gegenüber den heute im Metalleichtbau nicht mehr verwendeten Gewindeschneidschrauben - diese erzeugen ihr Muttergewinde spanabhebend einen besseren Formschluß mit den zu verbindenden Bauteilen und somit eine größere Lösefestigkeit und eine größere Tragfähigkeit bei einer Zugbeanspruchung in Richtung ihrer Achse. Die Anforderung einer dichten Verbindung wird durch die Verwendung von Dichtscheiben mit aufvulkanisierten Elastomer-Dichtungen erfüllt, die unter dem Schraubenkopf angeordnet werden können.

Für gewindefurchende Schrauben haben sich zwei Gewindetypen durchgesetzt. Zum einen das für die Befestigung auf dünnen Metallunterkonstruktionen mit Bauteildicken bis $2 \mathrm{~mm}$ und für Holzunterkonstruktionen geeignete Grobgewinde (Typ A) und zum anderen das Feingewinde (Typ B) mit zulaufender Spitze oder mit Zapfen, welches für die Befestigung auf dickeren Metallunterkonstruktionen geeignet ist (Bild 1.4).

Für Bohrschrauben hingegen wird eine Vielzahl von Schraubentypen verwendet, die sich sowohl bezüglich ihrer Gewinde- als auch bezüglich ihrer Bohrspitzengeometrie unterscheiden können. Der Bereich der Gewindeaußendurchmesser für die üblicherweise verwendeten Bohrschrauben liegt aus montagetechnischen und wirtschaftlichen Gründen zwischen 4,2mm und 6,3mm, wobei die Gewinde- und Bohrspitzengeometrien sehr individuell auf die jeweiligen speziellen Anwendungsbereiche der Schrauben abgestimmt und normativ auch nicht geregelt sind.

Als Verbindungen werden im folgenden ausschließlich solche mit gewindeformenden Schrauben behandelt. Werden im folgenden die Ausdrücke „Schrauben“ oder „geschraubte 
Verbindungen" verwendet, so sind damit stets gewindeformende Schrauben und die mit diesen Schrauben ausgeführten Verbindungen gemeint. Weiterhin werden als Unterkonstruktionen ausschließlich Unterkonstruktionen aus Stahl oder Aluminium untersucht.

\subsection{Problemstellung und Ziel der Arbeit}

Die in der Leichtbauweise ausgeführten geschraubten Verbindungen können im allgemeinen durch Kräfte in Achsrichtung der Verbindungselemente (Zugbelastung), durch Kräfte rechtwinklig dazu (Querkraftbelastung) oder durch eine Kombination dieser beiden Belastungsarten beansprucht werden.

Die Bemessung der Verbindungen erfolgt in der Regel auf der Grundlage von Tragfähigkeitsversuchen, die für jedes spezielle Verbindungselement erforderlich sind. Aus den Ergebnissen der Versuche werden dann Beanspruchbarkeiten für die Verbindungen abgeleitet.

Für Verbindungen in Konstruktionen mit Stahlblechprofilen geht die Konzeption für den Aufbau, die Durchführung und die Auswertung dieser Versuche auf [5] zurück. Das Versuchskonzept wurde in den Normentwurf DIN 18807-4 übertragen, dessen weitere Bearbeitung seitens der Bauaufsicht mittlerweile eingestellt wurde. Auf europäischer Ebene wurde in [6] ein ähnliches Versuchskonzept zur Herleitung der Beanspruchbarkeiten für geschraubte Verbindungen festgelegt.

Die Beanspruchbarkeiten der Verbindungen werden nach diesem Versuchskonzept über unterschiedliche Versuchsanordnungen getrennt nach Beanspruchungsarten - Querkraftoder Zugbeanspruchung - und für die Zugbeanspruchung auch noch getrennt nach den möglichen Versagensarten der zu verbindenden Bauteile - Versagen des unter dem Schraubenkopf anliegenden Bauteils durch ein Durchknöpfen des Schraubenkopfes durch dieses Bauteil oder Versagen durch einen Auszug der Schraube aus der Unterkonstruktion - ermittelt. Weiterhin werden Versuche zur Bestimmung der Zugbruch- und der Scherbruchtragfähigkeiten der Schrauben durchgeführt.

Für Verbindungen in Konstruktionen mit Kaltprofilen aus Stahlblech und für Verbindungen von Sandwichelementen mit Deckschichten aus Stahl sind die Beanspruchbarkeiten, die sich aus diesen Versuche ergeben, den entsprechenden allgemeinen bauaufsichtlichen Zulassungen [4] und [3] zu entnehmen.

Für Aluminium-Trapezprofile regelt DIN 18807-7, wie die Beanspruchbarkeiten der Verbindungen über Versuche zu ermitteln sind. Diese Regelungen stimmen weitgehend mit dem Versuchskonzept aus [5] überein. Die aus Versuchen ermittelten Beanspruchbarkeiten für Verbindungen von Aluminium-Trapezprofilen werden in Form von allgemeinen bauaufsichtlichen Prüfzeugnissen wiedergegeben. 
Die Beanspruchbarkeiten für Verbindungen von Sandwichelementen mit Deckschichten aus Aluminium werden ebenfalls über Versuche gemäß [5] oder DIN 18807-7 ermittelt. Diese Beanspruchbarkeiten werden in den allgemeinen bauaufsichtlichen Zulassungen der Sandwichelemente angegeben.

In [7] wurde erstmals versucht, die Tragfähigkeit querkraftbeanspruchter Verbindungen von Stahlblechen mit Hilfe von Blechschrauben (Schrauben mit Gewindeformen wie sie bei gewindefurchenden Schrauben (Bilder 1.4b) und 1.4c) vorliegen) formelmäßig zu beschreiben. Die empirisch abgeleitete Formel gilt jedoch nur für Verbindungen von Stahlblechen gleicher Dicke und Festigkeit und mit einem Durchgangsloch im oberen Bauteil und ist daher für die Anwendungsbedingungen des Leichtbaus nicht mehr relevant.

In [8] werden für Verbindungen von Stahlprofilblechen auf Stahlunterkonstruktionen mit Hilfe von gewindefurchenden Schrauben vom Typ B (Bild 1.4b)) empirisch abgeleitete Bemessungsregeln für Querkraft- und Zugbeanspruchungen angegeben. Den Regeln für die Querkraftbeanspruchung liegen die in den durchgeführten Versuchen erreichten Bruchlasten zugrunde. Das gemäß [5] einzuhaltende Verformungskriterium für Querkraftverbindungen von maximal $3 \mathrm{~mm}$ ist daher durch die Bemessungsregeln nicht berücksichtigt. Durch dieses Verformungskriterium wird gemäß [5] sichergestellt, daß sich unter der ermittelten Querkraftbeanspruchbarkeit in dem dünneren Bauteil der Verbindung kein übermäßig großes Langloch ausbildet, durch welches die übertragbaren Längszugkräfte stark reduziert würden.

Im Eurocode 3-1.3 werden Formeln zur Dimensionierung von geschraubten Verbindungen von dünnwandigen Stahlblechen angegeben. Diese Formeln gelten sowohl für Bohrschrauben als auch für gewindefurchende Schrauben. Sie resultieren aus der statistischen Auswertung [9], [10] von Versuchen, die nach dem in [5] beschriebenen Versuchkonzept durchgeführt wurden. Die Bemessungsregeln zur Ermittlung der Querkraft- und der Durchknöpfbeanspruchbarkeit decken die in der Leichtbaupraxis üblichen Anwendungsbedingungen bezüglich Werkstoffestigkeiten und Blechdickenbereiche der verbundenen Bauteile ab. Die Anwendung der Bemessungsregel zur Ermittlung der Auszugsbeanspruchbarkeit ist jedoch auf Stahlunterkonstruktionen mit Blechdicken von mindestens $0,9 \mathrm{~mm}$ beschränkt. In [4] sind auch zugbeanspruchte Verbindungen mit einer minimalen Dicke der Stahlunterkonstruktion von 0,63mm aufgeführt. In [11], [12] und [13] wurden die im Eurocode 3-1.3 angegebenen Bemessungsregeln überprüft. Hierzu wurden zahlreiche Versuche ausgewertet, die im Rahmen des Zulassungsverfahrens zu [3] und [4] an der Versuchsanstalt für Stahl, Holz und Steine durchgeführt wurden. Als Ergebnis dieser Überprüfung wurde festgestellt, daß die Formeln zur Ermittlung der Querkraft- und der Auszugsbeanspruchbarkeit teilweise auf der unsicheren Seite liegen. Die daraufhin angepaßten Formeln in prEN 1993-1-3 (dem aktuellen Stand der überarbeiteten Version des Eurocode 3-1.3) tragen den in [11], [12] und [13] festgestellten Unsicherheiten Rechnung. Die Formel zur Ermittlung der Auszugsbeanspruchbar- 
keit konnte jedoch aufgrund des Fehlens eines mechanischen Modells nicht auf geringere Bauteildicken erweitert werden. In der ursprünglichen Formel des Eurocode 3-1.3 geht zur Ermittlung der Auszugsbeanspruchbarkeit als einziger Schraubenparameter nur der Gewindeaußendurchmesser ein. In [11] wurde festgestellt, daß die Auszugstragfähigkeit auch für den im Eurocode 3-1.3 definierten eingeschränkten Anwendungsbereich von der Gewindesteigung der Schraube beeinflußt wird. In prEN 1993-1-3 wurde der Einfluß der Gewindesteigung auf die Auszugstragfähigkeit daher über ein Abgrenzungskriterium implizit erfaßt. Der Einfluß der Gewindesteigung auf die Auszugstragfähigkeit wird auch durch die Untersuchungen in [14] bestätigt. Weitere Untersuchungen in [15] lassen vermuten, daß die Auszugstragfähigkeit auch durch die Größe des Kernloches beeinflußt wird, welches in der Unterkonstruktion durch die Schraubenmontage erzeugt wird.

In DIN 18807-6 sind für Aluminium-Trapezprofile Formeln zur rechnerischen Ermittlung der Beanspruchbarkeiten von geschraubten Verbindungen angegeben, die auf europäischer Ebene in prEN 1999-1-4 übernommen wurden. Während die Formeln zur Ermittlung der Querkraft- und Durchknöpfbeanspruchbarkeit sowohl für gewindefurchende Schrauben als auch für Bohrschrauben gelten, ist der Anwendungsbereich der Formel zur Ermittlung der Auszugsbeanspruchbarkeit auf gewindefurchende Schrauben (Bilder 1.4b) und 1.4c)) mit Gewindeaußendurchmesser im Bereich von $6,25 \mathrm{~mm}$ bis $6,50 \mathrm{~mm}$ beschränkt. In der in DIN 18807-6 angegebenen Formel für die Auszugsbeanspruchbarkeit geht - ebenso wie in der Bemessungsregel nach Eurocode 3-1.3 - als einziger Schraubenparameter nur der Gewindeaußendurchmesser ein. Die Formel ist gültig für Aluminiumunterkonstruktionen mit Blechdicken von mindestens $0,9 \mathrm{~mm}$ und für Stahlunterkonstruktionen mit Blechdicken von mindestens 0,75mm. Die nach Eurocode 3-1.3 ermittelte Auszugsbeanspruchbarkeit führt im Vergleich zu der nach DIN 18807-6 ermittelten Beanspruchbarkeit für den Anwendungsbereich, der durch beide Normen abgedeckt wird (gewindefurchende Schrauben mit Gewindeaußendurchmesser im Bereich von 6,25mm bis 6,50mm, Stahlunterkonstruktionen mit einer Blechdicke von mindestens 0,9mm), zu maximal 75\% größeren und 30\% kleineren Bemessungsergebnissen. Die nach prEN 1993-1-3 ermittelte Auszugsbeanspruchbarkeit liegt für diesen Anwendungsbereich maximal 20\% über und 30\% unter der nach DIN 18807-6 ermittelten Beanspruchbarkeit.

In [16] wurden anhand der Ergebnisse von Auszugversuchen mit gewindefurchenden Schrauben aus Aluminiumunterkonstruktionen charakteristische Werte für die Auszugsbeanspruchbarkeit abgeleitet. Diese Werte sind im Vergleich zu der nach DIN 18807-6 ermittelten Beanspruchbarkeit um etwa $10 \%$ größer.

In der schwedischen Norm StBK-N5 werden Bemessungsformeln angegeben, die sehr ähnlich aufgebaut sind wie die Formeln in DIN 18807-6. 
Diese Ausführungen zeigen, daß man bei der Bestimmung der Auszugsbeanspruchbarkeiten von geschraubten Verbindungen in der Leichtbauweise auf die Durchführung von Versuchen angewiesen ist. Zum einen sind die existierenden Formeln in ihrem Anwendungsbereich sowohl hinsichtlich der Schraubenarten als auch hinsichtlich der Blechdicken der Unterkonstruktion eingeschränkt. Zum anderen beschreiben die Formeln das Auszugstragverhalten der Verbindungen nur unzureichend. Der Grund hierfür ist, daß die bei der Herleitung der Formeln aus der statistischen Auswertung von Versuchen verwendeten einfachen mechanischen Modelle nicht alle für das Tragverhalten maßgebenden Einflußfaktoren berücksichtigen. Dies gilt in besonderer Weise für den Einfluß unterschiedlicher Schraubengeometrien auf die Auszugstragfähigkeit der Verbindungen. Es ist deshalb zumindest fraglich, ob durch die Formeln die große Bandbreite an unterschiedlichen Schraubentypen abgedeckt ist, die in der Leichtbauweise eingesetzt werden. Somit besteht einerseits die Gefahr, daß die Anwendung der Bemessungsformeln zu unsicheren Ergebnissen führt und andererseits können möglicherweise vorhandene Tragfähigkeitsreserven nicht voll ausgenutzt werden. Diese Situation wird dadurch verschärft, daß die Entwicklung auf dem Sektor der Verbindungsmitteltechnik nicht abgeschlossen ist, was vor allem für Bohrschrauben gilt, die normativ weder hinsichtlich ihrer Geometrie noch hinsichtlich des verwendeten Werkstoffes geregelt sind und die in [3] und [4] stetig durch neue Varianten ergänzt werden.

Ziel der vorliegenden Arbeit ist es, sichere und zugleich wirtschaftliche Bemessungsregeln für die Auszugstragfähigkeit von zugbeanspruchten gewindeformenden Schrauben abzuleiten. Die Grundlage dieser Formeln sollen dabei theoretische Modelle bilden, die aus der Kenntnis um die für das Tragverhalten der Verbindungen maßgebenden Einflußfaktoren resultieren.

\subsection{Gliederung der Arbeit}

Das im vorigen Abschnitt definierte Ziel, die Ableitung von sicheren und zugleich wirtschaftlichen Bemessungsregeln für die Auszugstragfähigkeit von zugbeanspruchten gewindeformenden Schrauben, soll durch die folgende Vorgehensweise erreicht werden.

In Kapitel 2 werden die grundlegenden Eigenschaften der Verbindungen erläutert, die im Rahmen dieser Arbeit untersucht werden. Dabei werden

- die sich aus unterschiedlichen Verbindungstypen ergebende Bandbreite an möglichen Blechdickenbereichen und Werkstoffen der Unterkonstruktion,

- die Geometrie und das Werkstoffverhalten der Schrauben sowie 
- die Versuche zur Bestimmung der Auszugstragfähigkeiten der Schrauben aus ihren Unterkonstruktionen und die aus diesen Versuchen resultierenden Einschränkungen bezüglich der Position der Verschraubung

beschrieben. Da es in der Leichtbauweise eine Vielzahl von unterschiedlichen Anwendungsmöglichkeiten für geschraubte Verbindungen gibt und die Schrauben weder hinsichtlich ihrer Geometrie noch hinsichtlich der verwendeten Werkstoffe genormt sind, dient dieses Kapitel zur Definition der Randbedingungen, die im weiteren Verlauf der Arbeit vorausgesetzt werden.

In Kapitel 3 wird eine Versuchsdatenbank vorgestellt, die erstellt wurde aus zahlreichen Versuchen, die an der Versuchsanstalt für Stahl, Holz und Steine im Rahmen des Zulassungsverfahrens zu [3] und [4] oder gemäß DIN 18807-7 durchgeführt wurden. Mit Hilfe dieser Daten werden zum einen die im Rahmen dieser Arbeit entwickelten FE-Modelle kalibriert und verifiziert (Kapitel 4) und zum anderen werden sie in Kapitel 5 dazu verwendet, um bei der Festlegung von Bemessungsregeln die Streuungen der die Tragfähigkeiten bestimmenden Einflußgrößen zu berücksichtigen.

In Kapitel 4 werden FE-Modelle entwickelt, mit deren Hilfe Parameterstudien durchgeführt werden, um die für das Tragverhalten der Verbindungen maßgebenden Einflußfaktoren zu qualifizieren und zu quantifizieren. Mit den Ergebnissen der Parameterstudien werden mechanische Modelle zur Beschreibung des Tragverhaltens der Verbindungen abgeleitet.

Mit Hilfe der mechanischen Modelle aus Kapitel 4 und der statistischen Auswertung der Daten der Versuchsdatenbanken werden im Kapitel 5 Bemessungsregeln für die Auszugsbeanspruchbarkeiten von gewindeformenden Schrauben aus Stahl- und Aluminiumunterkonstruktionen abgeleitet.

Kapitel 6 enthält schließlich eine Zusammenfassung der Arbeit. 


\section{Charakteristische Eigenschaften der untersuchten Verbin- dungen}

\subsection{Verbindungstypen und Versagensmechanismen}

Dasjenige Bauteil der Verbindung, das sich unter einer Zugbeanspruchung gegen den Schraubenkopf abstützt - dies können wie z. B. bei der Querstoßverbindung zweier benachbarter Profilbleche über einem Auflager auch mehrere Bauteile sein - wird im folgenden wie in der Baupraxis üblich als Bauteil I bezeichnet.

Das andere Bauteil der Verbindung - das lastaufnehmende Bauteil - wird als Unterkonstruktion oder als Bauteil II bezeichnet. Im Rahmen dieser Arbeit wird vorausgesetzt, daß die Unterkonstruktion ausschließlich durch ein Bauteil gebildet wird.

Eine auf die Verbindung wirkende Zugbeanspruchung kann aus dem Lastfall Windsog, aus abhebenden Kräften bei Durchlaufwirkung oder aber aufgrund einer horizontalen Beanspruchung und einem als Schubfeld wirkenden Profilblechs aus der Umlenkung des Schubflusses - hier in Kombination mit einer Beanspruchung rechtwinklig zur Schraubenachse - resultieren (vgl. [17] - [20] und DIN 18807-1, -3, -8, -9).

Der im Rahmen dieser Arbeit untersuchte Versagensfall des Auszugs der Schraube aus der Unterkonstruktion kann sich durch ein Versagen des in der Unterkonstruktion von der Schraube geformten Muttergewindes, durch ein Versagen des Schraubengewindes oder durch eine Kombination dieser beiden Versagensmechanismen einstellen. Zur formelmäßigen Beschreibung der Auszugstragfähigkeit sind daher in erster Linie die charakteristischen Eigenschaften der Schrauben (vgl. Abschnitt 2.2) sowie der Unterkonstruktionen von Interesse. Da einerseits diese Eigenschaften durch die gesamte Konstruktion beeinflußt werden und andererseits auch die Tragfähigkeit einer zugbeanspruchten Verbindung durch eventuell auftretende Wechselwirkungen zwischen Bauteil I und Bauteil II beeinflußt wird (vgl. Abschnitt 2.3), muß in zweiter Linie auch das Bauteil I - und damit die gesamte Verbindung betrachtet werden.

Anhand der im Bild 2.1 dargestellten typischen Konstruktion eines zweischaligen Dach- oder Wandaufbaus im Leichtbau können die möglichen Verbindungstypen aufgezeigt werden.

Die aus diesen Verbindungstypen resultierende Bandbreite an bisher üblichen Blechdicken, Werkstoffeigenschaften und Querschnittsformen für die Unterkonstruktionen sind in der Tabelle 2.1 zusammengefaßt. 


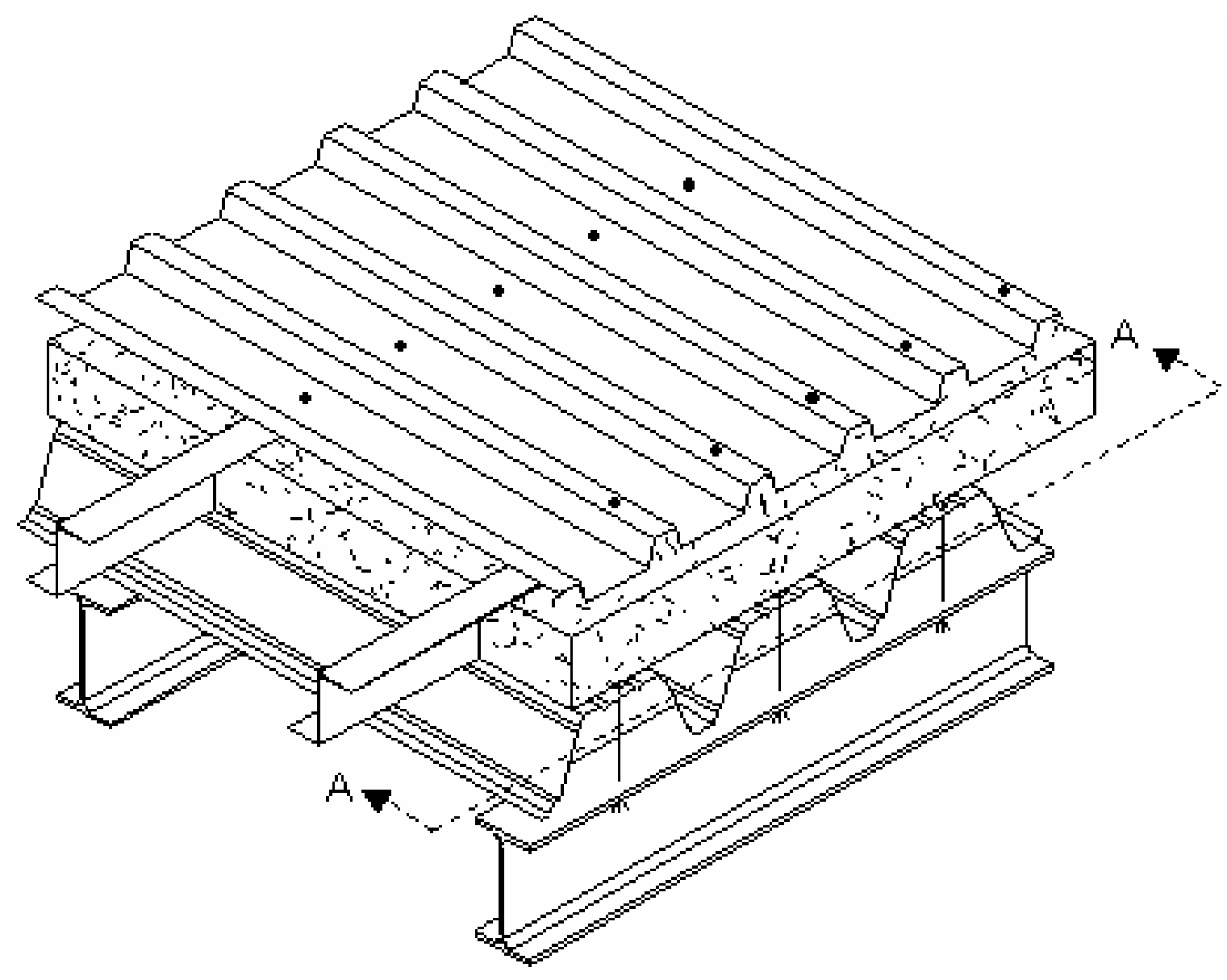

Querschnitt A-A:

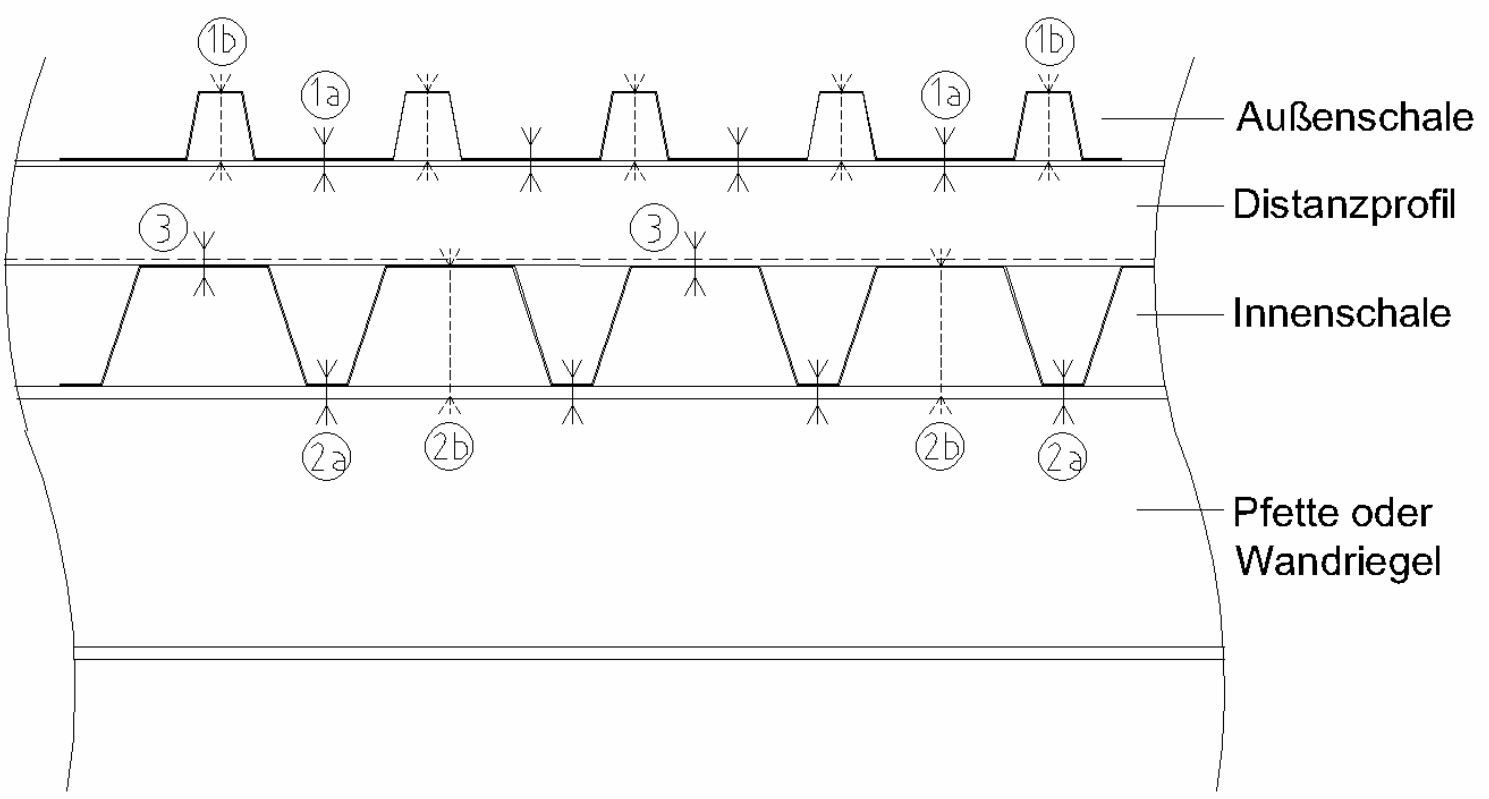

Bild 2.1: Zweischaliger Dach- oder Wandaufbau 
Tabelle 2.1: Charakteristische Eigenschaften der Unterkonstruktionen

\begin{tabular}{|c|c|c|c|c|c|}
\hline \multirow[b]{3}{*}{$\begin{array}{l}\text { Verbin- } \\
\text { dungstyp } \\
\text { gemäß } \\
\text { Bild } 2.1\end{array}$} & \multicolumn{5}{|c|}{ Charakteristische Eigenschaften der Unterkonstruktion } \\
\hline & Wer & kstoffe & & & \\
\hline & $\begin{array}{c}\text { Stahl: } \\
\text { Festigkeitsklassen } \\
\text { gemäß: } \\
\text { DIN EN 10025-2 } \\
\text { u. } \\
\text { DIN EN } 10326\end{array}$ & $\begin{array}{c}\text { Aluminium: } \\
\text { Mindestzugfestig- } \\
\text { keit: } \\
\mathrm{R}_{\mathrm{m}, \min }\left[\mathrm{N} / \mathrm{mm}^{2}\right] \\
\text { Mindestwert der } \\
0,2 \% \text {-Dehngrenze: } \\
\mathrm{R}_{\mathrm{P} 0,2, \min }\left[\mathrm{N} / \mathrm{mm}^{2}\right] \\
\end{array}$ & $\begin{array}{c}\text { Blechdicke } \\
\qquad \mathrm{t}_{\|} \\
{[\mathrm{mm}]}\end{array}$ & $\begin{array}{l}\text { Typische } \\
\text { Quer- } \\
\text { schnitts- } \\
\text { form }\end{array}$ & $\begin{array}{l}\text { Funktion für } \\
\text { die Kon- } \\
\text { struktion }\end{array}$ \\
\hline $1 a, 1 b$ & $\begin{array}{c}\text { S235 } \\
\text { DIN EN 10025-2, } \\
\text { für } \mathrm{t}_{\|} \leq 3 \mathrm{~mm} \text { auch: } \\
\text { S280, S320 } \\
\text { DIN EN } 10326\end{array}$ & $\begin{array}{c}\text { z. B. EN AW-6060; } \\
\text { EN AW-6005A } \\
\text { nach DIN EN 755-2: } \\
215 \leq R_{\mathrm{m}, \min } \leq 250 \\
160 \leq \mathrm{R}_{\mathrm{P} 0,2, \min } \leq 200\end{array}$ & $1,5-6$ & 7 & $\begin{array}{l}\text { Distanz- } \\
\text { konstruk- } \\
\text { tion }\end{array}$ \\
\hline $2 a, 2 b$ & $\begin{array}{c}\text { S235 } \\
\text { DIN EN 10025-2 }\end{array}$ & $\begin{array}{c}\text { z. B. EN AW-6060; } \\
\text { EN AW-6005A } \\
\text { nach DIN EN 755-2: } \\
215 \leq R_{m, \min } \leq 250 \\
160 \leq R_{P 0,2, \min } \leq 200\end{array}$ & $5-14$ & & $\begin{array}{l}\text { Trag- } \\
\text { konstruk- } \\
\text { tion }\end{array}$ \\
\hline 3 & $\begin{array}{c}\text { S280, S320 } \\
\text { DIN EN } 10326\end{array}$ & $\begin{array}{l}\text { z. B. EN AW-3005; } \\
\text { EN AW-3105 } \\
\text { nach DIN EN 485-2: } \\
195 \leq R_{\mathrm{m}, \min } \leq 220 \\
160 \leq \mathrm{R}_{\mathrm{P} 0,2, \min } \leq 190\end{array}$ & $0,50-1,50$ & & $\begin{array}{l}\text { Raumab- } \\
\text { schluß und } \\
\text { Auflageflä- } \\
\text { che für die } \\
\text { Wärme- } \\
\text { dämmung }\end{array}$ \\
\hline
\end{tabular}

Die Eigenschaften der Unterkonstruktionen für Verbindungen mit Sandwichelementen sind in der Tabelle 2.1 durch die Verbindungstypen 1a, 1b, 2a und 2b mit abgedeckt.

Die in der Tabelle 2.1 angegebenen Obergrenzen für die Werkstoffestigkeit und Blechdicke der Unterkonstruktion - also Stahl der Festigkeitsklasse S235 mit einer Blechdicke bis zu $14 \mathrm{~mm}$ sowie Stahl der Festigkeitsklasse S320 mit einer Blechdicke bis zu 3mm - entsprechen der zur Zeit gängigen Praxis der Leichtbauweise. Dies wird durch die allgemeinen bauaufsichtlichen Zulassungen [3] und [4] deutlich, in denen ausschließlich Unterkonstruktionen dieser Festigkeitsklassen und dieses Blechdickenbereiches für Verbindungen mit gewindeformenden Schrauben geregelt sind. Diese Obergrenzen stellen insbesondere auch für die gewindeformenden Schrauben aufgrund ihrer charakteristischen geometrischen und mechanischen Eigenschaften (vgl. Abschnitt 2.2) die zur Zeit bestehenden Anwendungsgrenzen dar. Aufgrund der Bestrebungen hin zu einer immer wirtschaftlicheren Bauweise und der sich daraus ergebenden Forderung nach immer leichteren Konstruktionen aus höherfesten Werkstoffen ist auch für die Zukunft der Leichtbauweise eine Ausweitung auf Unterkonstruktionen 
aus höherfesten Werkstoffen zu erwarten. Für den Verbindungstyp 3 in der Tabelle 2.1, in dem die Unterkonstruktion durch ein dünnwandiges Profilblech gebildet wird, zeigt sich dieser Trend bereits deutlich daran, daß Stahlprofilbleche immer häufiger in der Festigkeitsklasse S350 hergestellt werden [21] - [24]. In [21] ist sogar ein Profilblech mit einer Nennblechdicke von 0,55mm aufgeführt, das aus Stahl der Festigkeitsklasse S550 hergestellt wird. Für Unterkonstruktionsdicken größer 1,5mm gibt es darüber hinaus erste Bestrebungen der Verbindungselementhersteller gewindeformende Schrauben zu entwickeln, die auch für Stahl der Festigkeitsklasse S355 geeignet sind.

Zur Berücksichtigung der zukünftigen Entwicklungen im Leichtbau wurde im Rahmen der durchgeführten FE-Parameterstudien (vgl. Kapitel 4) als Obergrenze für die Werkstoffestigkeit der Unterkonstruktion ein Stahl der Festigkeitsklasse S355 untersucht.

\subsection{Schrauben}

\subsubsection{Zusammenhang zwischen Schraubengeometrie, Montagevorgang und Anwen- dungsbereich}

Die Geometrie einer gewindeformenden Schraube läßt sich über ihre Länge hinsichtlich der unterschiedlichen Aufgaben, die an die Schraube während der Montage und im Beanspruchungszustand der Verbindung gestellt werden, in verschiedene Zonen unterteilen. Die geometrische Gestaltung der einzelnen Zonen ist abhängig von dem vorgesehenen Anwendungsbereich der Schraube (Blechdickenbereich der zu verbindenden Bauteile, Werkstoffkennwerte der zu verbindenden Bauteile, Art der Verbindung (direkt übereinanderliegende Bauteile oder Verbindung von auseinanderliegenden Bauteilen)) und ergibt sich für einen definierten Anwendungsbereich aus der Berücksichtigung und Abwägung der drei Gesichtspunkte Montagesicherheit, Tragfähigkeit und Ästhetik, wobei der letztgenannte Gesichtspunkt von untergeordneter Bedeutung ist und lediglich bei der Wahl der Kopfform zum tragen kommt.

Die wesentlichen funktionalen Zonen der gewindeformenden Schrauben lassen sich am Beispiel einer Bohrschraube, deren Anwendungsbereich in der Verbindung von direkt übereinanderliegenden Bauteilen liegt, aufzeigen (Bild 2.2). Die Schraube weist über ihre Länge sechs charakteristische Zonen auf [7], [25]:

- die Bohrspitze (Zone (1) im Bild 2.2),

- $\quad$ ein sich an die Bohrspitze anschließender gewindefreier Bereich (Zone (2) im Bild 2.2),

- $\quad$ der Gewindeeinlauf (Zone (3) im Bild 2.2),

das vollständig ausgeformte Schraubengewinde (Zone (4) im Bild 2.2), 
der Gewindeauslauf einschließlich dem Übergangbereich vom Schaft zum Schraubenkopf (Zone (5) im Bild 2.2) und

der Schraubenkopf (Zone (6) im Bild 2.2).

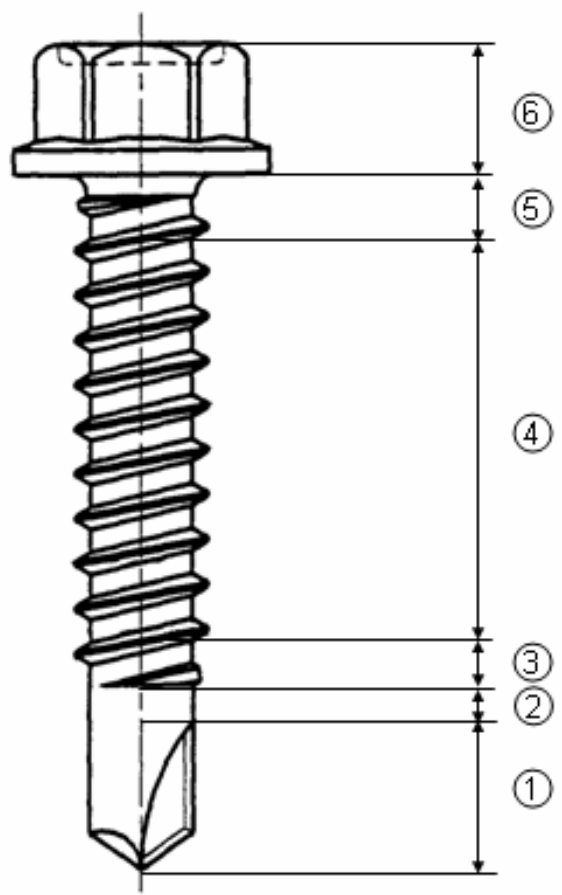

Bild 2.2: Charakteristische Zonen einer Bohrschraube zur Verbindung von direkt übereinanderliegenden Bauteilen

Während der Montage der im Bild 2.2 dargestellten Bohrschraube treten folgende im Bild 2.3 dargestellte Drehmomente in den Schraubenzonen (1) bis (5) auf:

Das Bohrmoment $M_{B}$ ist das Moment, das aufgebracht werden muß, um mit der Bohrspitze in den Bauteilen I und II ein Kernloch zu erzeugen. Es ist von allen nachfolgend beschriebenen Momenten das kleinste.

- Das Einschraubmoment $M_{E}$ ist das maximale Moment, das während des Gewindeformvorgangs in der Zone (3) aufgebracht werden muß, damit die Schraube in den zu verbindenden Bauteilen ihr Gegengewinde durch eine Stauchung und Verdrängung von Volumen spanlos formt. Es tritt gerade dann auf, wenn der erste vollständig ausgeformte Gewindegang der Schraube (treibender Gewindegang) am Übergang zwischen den Zonen (3) und (4) aus der Unterkonstruktion austritt. An diesem Punkt ist das Gegengewinde getrieben. Durch die nachfolgenden Gewindegänge tritt noch ein Aufweiten des Gegengewindes ein, was sich in einem kontinuierlichen Abfall des Drehmomentenverlaufs äußert.

- Das Überdrehmoment $M_{u ̈, I}$ ist das Moment, das beim Anziehen der Verbindung im Gewindeauslauf der Schraube (Zone (5)) gerade aufgebracht werden kann, ohne daß das von der Schraube im Bauteil I geformte Gegengewinde aufgrund einer zu großen Torsionsbeanspruchung versagt. Das Überschreiten dieses Grenzmomentes während des 
Anziehvorgangs stellt sicher, daß im Bauteil I ein Durchgangsloch erzeugt wird, so daß danach das Bauteil II fest gegen das Bauteil I verspannt werden kann.

- Das Überdrehmoment $M_{u ̈, ı}$ ist das Moment, das beim Anziehen der Verbindung im voll ausgeformten Gewindebereich der Schraube (Zone(4)) gerade aufgebracht werden kann, bevor das von der Schraube in der Unterkonstruktion geformte Muttergewinde aufgrund einer zu großen Torsionsbeanspruchung versagt.

- Das Anziehmoment $M_{A}$ ist das Moment, das zur Vorspannung der Verbindung aufgebracht wird. Es muß zwischen dem Überdrehmoment $M_{u ̈, I}$ und dem Überdrehmoment $M_{u ̈, I I}$ liegen. Bei den Überdrehmomenten $M_{u ̈, I}$ und $M_{u ̈, I I}$ handelt es sich im Gegensatz zu den anderen Momenten (Widerstände) um Beanspruchbarkeiten.

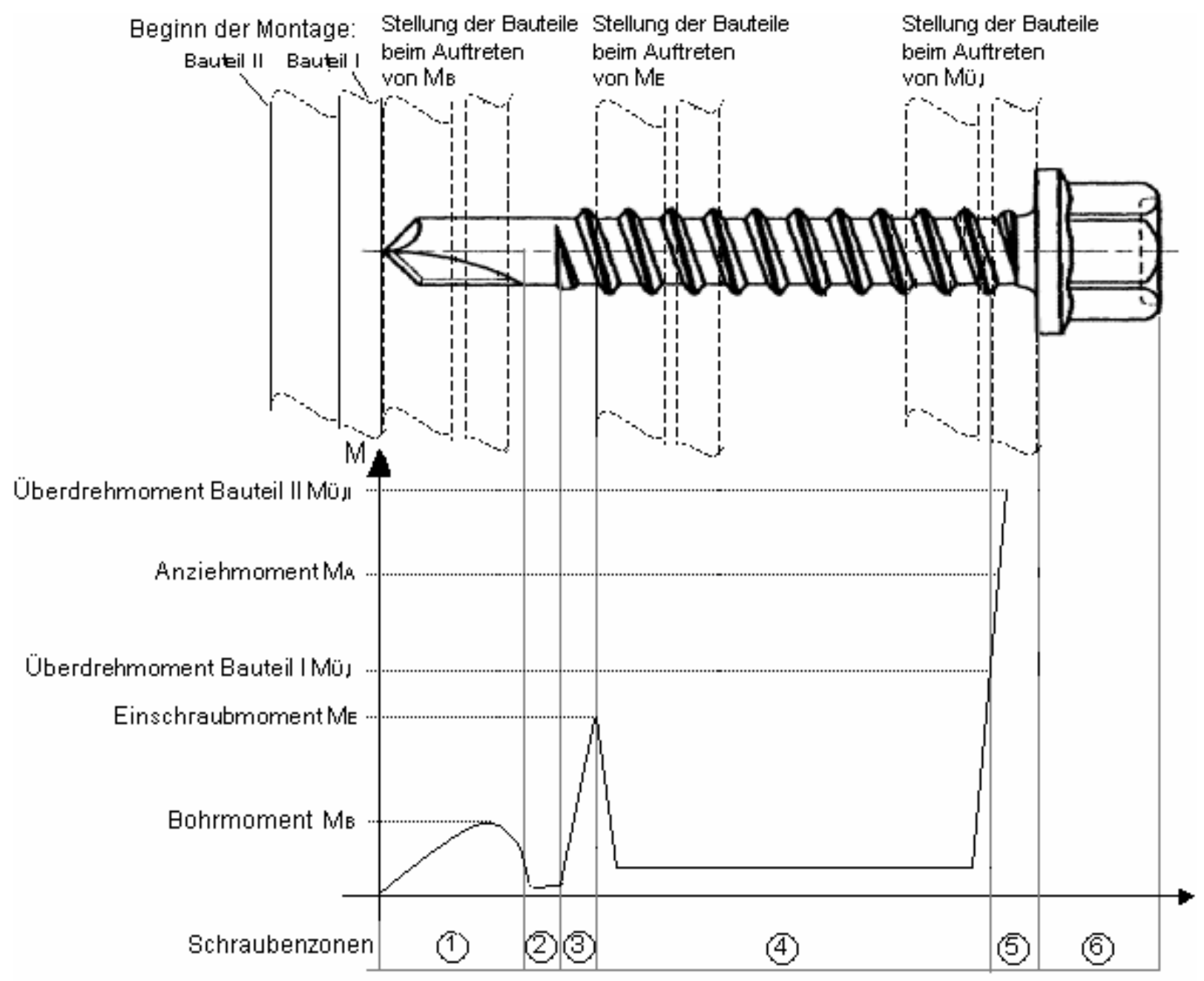

Bild 2.3: Drehmomente während der Montage von Bohrschrauben

Die Zusammenhänge zwischen der Schraubengeometrie, der Größe der während der Schraubenmontage auftretenden Drehmomente und den möglichen Anwendungsbereich für eine Schraube werden nachfolgend für die einzelnen im Bild 2.2 dargestellten funktionalen Schraubenzonen verdeutlicht. 


\section{Bohrspitze, Zone (1) im Bild 2.2:}

Die Bohrspitze hat die Aufgabe, in den zu verbindenden Bauteilen das erforderliche Kernloch spanabhebend zu erzeugen. Um diese Aufgabe erfüllen zu können, muß die Bohrspitze grundlegend derart ausgebildet werden, daß während des gesamten Bohrvorgangs ein Spantransport nach außen sichergestellt ist. Ist diese Bedingung nicht erfüllt, so kommt es zu einem „Spänestau“, der den Bohrvortrieb behindert und somit dazu führen kann, daß die Bohrspitze durch die aus der dann übermäßigen Reibung entstehenden Wärme verglüht [25]. Für die Ausführung der Bohrspitze haben sich zwei Techniken auf dem Markt durchgesetzt. Dies ist zum einen die in Bild 2.2 bereits dargestellte massive Bohrspitze mit einer eingearbeiteten Spannut (auch Flut genannt) und zum anderen ein Bohrplättchen, welches im Kern der Schraube verankert wird (Bild 2.4).
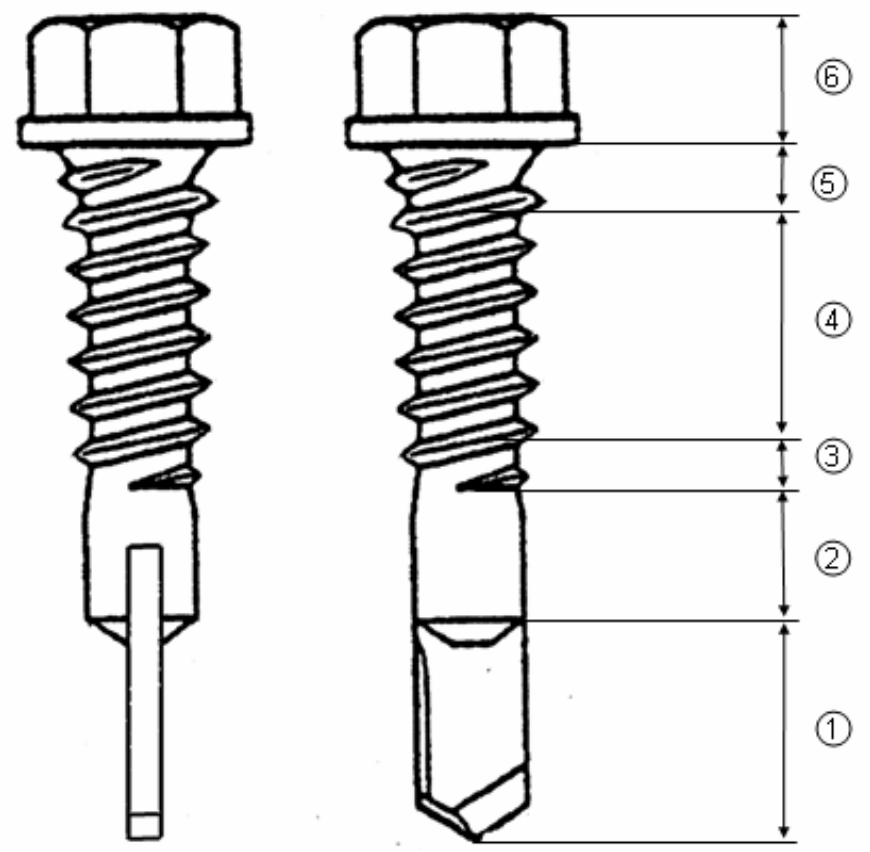

Bild 2.4: Bohrschraube mit einer als Bohrplättchen ausgebildeten Bohrspitze

Die Länge der Bohrspitze muß für beide Ausführungsvarianten zur Gewährleistung des erforderlichen Spantransportes nach außen mindestens so groß sein, wie die Summe der direkt übereinanderliegenden Blechdicken der Verbindung. Die Länge der Bohrspitze begrenzt damit die maximal mögliche Gesamtblechdicke der Verbindung.

Ein weiterer wesentlicher Gestaltungsparameter der Bohrspitze ist ihr Durchmesser. Die Größe dieses Durchmessers bestimmt den Durchmesser des Kernloches, das während des Bohrvorgangs in den Bauteilen der Verbindung erzeugt wird, und beeinflußt somit über die Gestalt des in diesen Bauteilen geformten Gegengewindes sowohl die im Rahmen dieser Arbeit untersuchte Auszugstragfähigkeit der Schraube aus der Unterkonstruktion als auch die Größe aller Drehmomente, die während der Montage der Verbindung in den unterschiedlichen Schraubenzonen auftreten (Bild 2.3). 
Das Moment $\mathrm{M}_{\mathrm{B}}$ hat für die Wahl eines dem vorgesehenen Anwendungsbereich der Schraube geeigneten Bohrspitzendurchmessers keine wesentliche Bedeutung, da es von allen Momenten das kleinste ist. Die Festlegung für den Bohrspitzendurchmesser ergibt sich vielmehr aus dem Bestreben, ein möglichst großes Überdrehmoment $\mathrm{M}_{\mathrm{U}, \mathrm{II}}$ in der Unterkonstruktion zu erzielen - was zu einer großen Auszugstragfähigkeit der Schraube aus der Unterkonstruktion führt -, ohne den für ein einwandfreies Gewindeformen notwendigen Grenzwert des Einschraubmomentes $M_{E}$ zu überschreiten.

Ein großes Überdrehmoment $\mathrm{Mü,II}_{\text {Ist }}$ anzustreben, weil

- sich mit größer werdendem Überdrehmoment $M_{u ̈, I I}$ und gleichbleibendem Anziehmoment $M_{A}$ das Risiko vermindert, daß das Muttergewinde in der Unterkonstruktion während des Anziehvorgangs zerstört wird, und

- weil die Verbindung mit größer werdendem Überdrehmoment $M_{u ̈, I l}$ auch mit einem größeren Anziehmoment $M_{A}$ vorgespannt werden kann, was zu einer größeren Steifigkeit der Verbindung unter einer Zugbeanspruchung und zu einer größeren Lösesicherheit der Schraube unter einer wiederholten Beanspruchung führt.

Das Überdrehmoment $M_{u ̈, I I}$ nimmt zu, wenn

- die Werkstoffestigkeit oder die Blechdicke der Unterkonstruktion zunimmt, oder

- wenn die Verzahnung zwischen dem Muttergewinde in der Unterkonstruktion und dem Schraubengewinde zunimmt.

Eine größere Verzahnung zwischen Schrauben- und Muttergewinde kann durch eine Verkleinerung des Bohrspitzendurchmessers - bis zu einem unteren Grenzwert des Bohrspitzendurchmessers, bei dem in den zu verbindenden Bauteilen ein Kernloch erzeugt wird, dessen Durchmesser dem Kerndurchmesser der Schraube entspricht - erzielt werden (Bild 2.5).
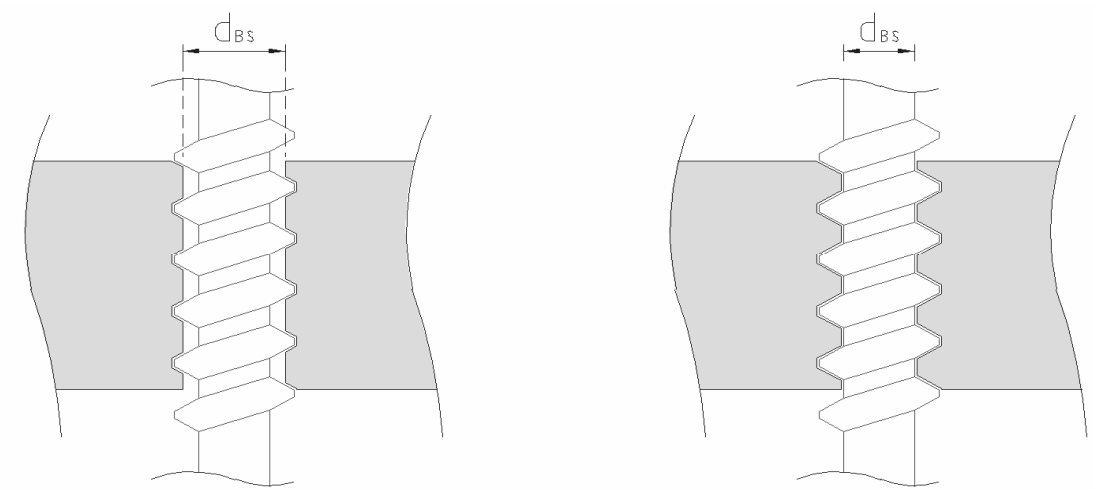

Bild 2.5: Zunehmende Verzahnung der Schraube in der Unterkonstruktion mit kleiner werdendem Bohrspitzendurchmesser $\mathrm{d}_{\mathrm{Bs}}$

Das Einschraubmoment $M_{E}$ wächst proportional mit steigender Volumenarbeit, die von der Schraube während des Gewindeformens verrichtet werden muß. Dies bedeutet, die Größe 
des Einschraubmomentes $M_{E}$ nimmt mit kleiner werdendem Bohrspitzendurchmesser sowie mit steigender Werkstoffestigkeit und Blechdicke der Verbindung zu und wird somit von den gleichen Parametern auf die selbe Weise beeinflußt, wie das Überdrehmoment Mü,Il. Um eine Deformation des Schraubengewindes, ein Ablösen der Gewindeflanken vom Schraubenkern oder sogar einen Torsionsbruch der Schraube während des Gewindeformvorgangs zu vermeiden, muß das Einschraubmoment $M_{E}$ einen ausreichenden Sicherheitsabstand $z u$ dem Torsionsbruchmoment der Schraube aufweisen. Zur Begrenzung des Einschraubmomentes ist es deshalb notwendig, mit steigender Blechdicke und Werkstoffestigkeit der Verbindung auch den Bohrspitzendurchmesser zu vergrößern.

Aus den erläuterten Zusammenhängen ergeben sich für die in der Baupraxis auftretenden unterschiedlichen Anwendungsbereiche folgende Grenzbedingungen für den Bohrspitzendurchmesser einer Schraube:

Für die Verbindung von dünnwandigen Bauteilen - beispielsweise die Verbindung eines Profilblechs mit einer dünnwandigen Zwischenkonstruktion (Verbindungstypen 1b und 3 in Bild 2.1) - werden zur Erzielung eines möglichst großen Überdrehmomentes Mü,II Bohrschrauben verwendet, deren Bohrspitze einen Durchmesser aufweisen, der gleich oder sogar kleiner ist als der Kerndurchmesser der Schraube (Bild 2.6 (a)). Aus der notwendigen Begrenzung des Einschraubmomentes $M_{E}$ ergibt sich für solche Schrauben der bezüglich Blechdicke und Werkstoffestigkeit der Verbindung maximal mögliche obere Anwendungsbereich. Dieser liegt für Verbindungen von Bauteilen aus Stahl der Festigkeitsklasse S235 in Abhängigkeit von der Gewindegeometrie der Schraube bei einer maximal möglichen Gesamtblechdicke der Verbindung im Bereich von ca. $2 \mathrm{~mm}$ bis $3 \mathrm{~mm}$.

Umgekehrt werden für die Verbindung von Profilblechen auf warmgefertigten Stahlprofilen mit einer Dicke bis zu 14mm - Verbindungstyp 2a und 2b in Bild 2.1 - Bohrschrauben verwendet, deren Bohrspitzendurchmesser zur Reduzierung des Einschraubmomentes $M_{E}$ über dem Mittelwert aus Kerndurchmesser und Gewindeaußendurchmesser der Schraube liegen (Bild 2.6 (b)). Für solche Bohrschrauben ergibt sich aus der Forderung nach einem ausreichend großen Überdrehmoment $M_{u ̈, „ I}$ der bezüglich Blechdicke und Werkstoffestigkeit der Verbindung noch sinnvolle untere Anwendungsbereich. Dieser untere Anwendungsbereich wird mit Sicherheit unterschritten, sofern die Blechdicke der Verbindung die sogenannte „Taumelgrenze“ erreicht, bei der die Schraube in das vorgebohrte Kernloch eingebracht werden kann, ohne daß ein Volumen umgeformt werden muß (vgl. [7] und DIN 7975). Dies bedeutet, daß sowohl das Einschraubmoment $M_{E}$ aber auch gleichzeitig das Überdrehmoment $M_{u ̈, I I}$ beim Erreichen der Taumelgrenze Null werden. Die Taumelgrenze existiert nur, sofern die Verbindung einen Kernlochdurchmesser aufweist, der größer ist als der Mittelwert aus Kerndurchmesser und Gewindeaußendurchmesser der Schraube. Sie kann über geometrische Betrachtungen analytisch hergeleitet werden [7]. 


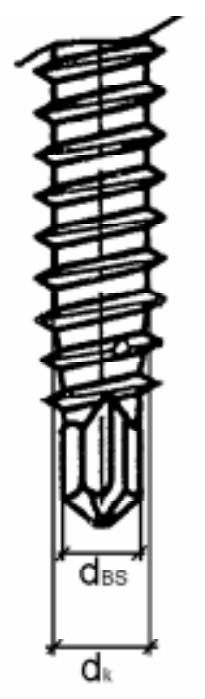

(a)

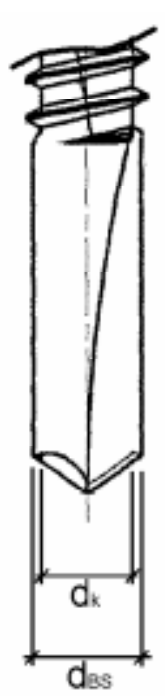

(b)

Bild 2.6: Bohrschrauben mit reduzierter (a) und mit breiter Bohrspitze (b)

Die Abhängigkeit zwischen dem Bohrspitzendurchmesser und dem möglichen Anwendungsbereich einer Schraube erklärt die Vielzahl an unterschiedlichen Bohrschraubentypen, die in der Praxis zur Anwendung kommen (vgl. [3] und [4]) und die sich bei sonst gleichen Abmessungen häufig nur in der Bohrspitzengeometrie unterscheiden können.

\section{An die Bohrspitze anschließender gewindefreier Bereich, Zone (2) im Bild 2.2:}

Die Zone (2) hat die Aufgabe, ein Distanzstück zu schaffen, das die beiden Arbeitsgänge „Kernloch bohren“ und „Gewinde formen“ deutlich voneinander trennt. Die Trennung dieser beiden Arbeitsgänge ist einerseits nötig, damit der unterschiedliche Schraubenvorschub beim Bohren und Gewindeformen überbrückt werden kann, und andererseits hat die zeitliche Trennung der beiden Arbeitgänge den positiven Effekt, daß die Beanspruchung für die Schraube während des Montagevorgangs minimiert wird, da sich die beim Bohren und Gewindeformen auftretenden Drehmomente nicht addieren können. Die Zone (2) ist vor allem bei solchen Schrauben deutlich ausgeprägt, die für die Verbindung auf dünnwandigen Unterkonstruktionen eingesetzt werden (Verbindungstyp 3 in Bild 2.1), da für solche Verbindungen die Gefahr besteht, daß das untere Blech aufgrund des Axialdrucks, der während des Bohrvorgangs auf die Schraube aufgebracht werden muß, und aufgrund der Nachgiebigkeit der dünnen Unterkonstruktion vom oberen weggedrückt wird.

\section{Gewindeeinlauf, Zone (3) im Bild 2.2:}

Die Zone (3) leitet vom fertigungsbedingten Gewindeanfang in das vollständig ausgeformte Schraubengewinde (Zone (4)) über. In dieser Zone wird das Gegengewinde in den zu verbindenden Bauteilen langsam ausgeformt, was sich in einem kontinuierlichen Anstieg des Drehmomentenverlaufs bis zum Erreichen des maximalen Einschraubmoments $M_{E}$ äußert. Durch die Form der Zone (3) wird nach [7] die Größe des Einschraubmomentes $M_{E}$ nicht beeinflußt, wohl aber die Steilheit des Drehmomentenverlaufs, die größer wird, wenn die Länge der Zone (3) kleiner wird. 


\section{Vollständig ausgeformtes Schraubengewinde, Zone (4) im Bild 2.2:}

Im Rahmen dieser Arbeit wird davon ausgegangen, daß nach der Montage der Verbindung die Unterkonstruktion innerhalb dieses Bereiches anliegt. Dies bedeutet, daß für eine zugbeanspruchte Verbindung die Lasteinleitung auf die Unterkonstruktion über das vollständig ausgeformte Schraubengewinde erfolgt. Mit der Erfüllung dieser Voraussetzung liegen die von einer Bohrschraube vorgegebenen geometrischen Randbedingungen, die ihre Auszugstragfähigkeit beeinflussen können, ausschließlich in der Ausbildung ihres Bohrspitzendurchmessers und der Gewindegeometrie der Zone (4). Diese Gewindegeometrie (Bild 2.7) wird eindeutig festgelegt durch den Gewindeaußendurchmesser $d_{g}$, die Gewindesteigung $P$, den Kerndurchmesser $d_{k}$, den Flankenwinkel $\alpha$ und die Abflachung $c$ des Gewindeprofils - oder anstelle von $\mathrm{c}$ die Basis $\mathrm{b}$ des Gewindeprofils.
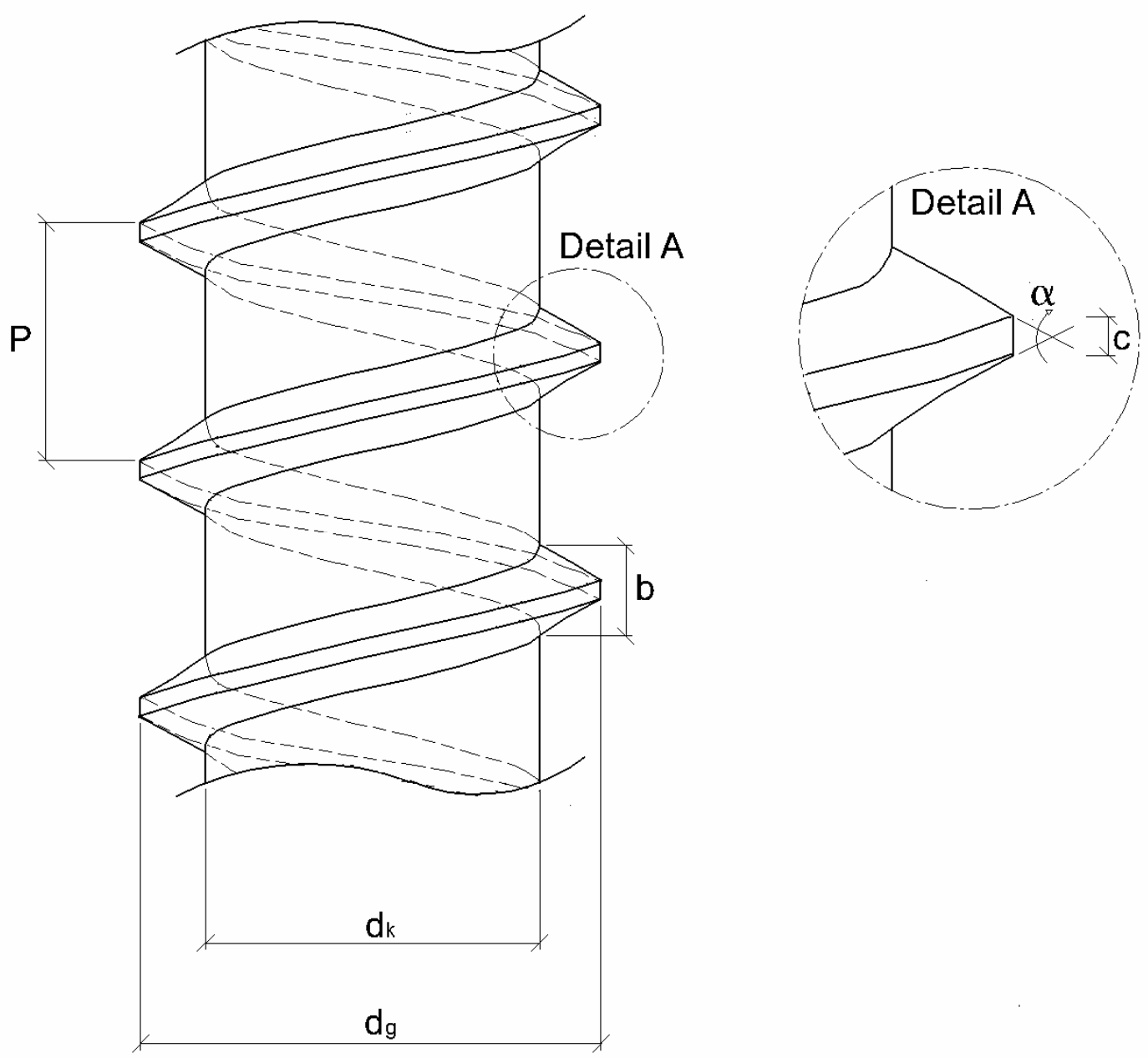

Bild 2.7: Geometrie des vollständig ausgeformten Gewindebereichs

Als gemeinsames charakteristisches Merkmal besitzen gewindeformende Schrauben - also sowohl Bohrschrauben als auch gewindefurchende Schrauben - eine offene Gewindeform mit einem Verhältnis $b / P<1$ und einem nahezu ideal dreieckigen Gewindeprofil mit einem Flankenwinkel von 60 (in [7], [26] und DIN EN ISO 1478 als Blechschraubengewinde be- 
zeichnet). Die Abflachung $\mathrm{c}$ des Gewindeprofils ist fertigungsbedingt und liegt im Bereich zwischen $0,10 \mathrm{~mm}$ und $0,15 \mathrm{~mm}$ (vgl. DIN EN ISO 1478).

Analog den obigen Ausführungen zum Bohrspitzendurchmesser führt eine Änderung der Gewindegeometrie einer Schraube, die eine größere Verzahnung zwischen der Schraube und den zu verbindenden Bauteilen zur Folge hat - also ein größer werdender GewindeauBendurchmesser oder eine kleiner werdende Gewindesteigung -, zu einem Anstieg der Aus-

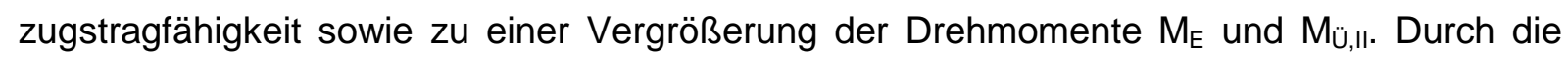
Kombination aus Bohrspitzen- und Gewindegeometrie wird also zusätzlich zu der Auszugstragfähigkeit auch im wesentlichen Maße der Anwendungsbereich für die Schraube festgelegt. Die Gewindeaußendurchmesser für die Bohrschrauben, die in der Leichtbauweise zum Einsatz kommen, liegen fast ausschließlich im Bereich zwischen 4,2mm und 6,3mm mit Nennwerten von 4,2mm, 4,8mm, 5,5mm und 6,3 mm (vgl. [3] und [4]). Obwohl für diesen Durchmesserbereich genormte Gewindeformen in DIN EN ISO 1478 spezifiziert werden, müssen die für die Baupraxis verwendeten Bohrschrauben aus den nachfolgend angegebenen Gründen als nicht genormte Verbindungselemente angesehen werden:

Die Schraubenhersteller müssen sich nicht notwendigerweise an die Normvorgaben aus DIN EN ISO 1478 halten, da der Verwendbarkeitsnachweis einer Bohrschraube für einen definierten Anwendungsbereich sowie der Tragfähigkeitsnachweis für die mit einer Schraube ausgeführte Verbindung bisher in Deutschland über allgemeine bauaufsichtliche Zulassungen [3], [4] oder allgemeine bauaufsichtliche Prüfzeugnisse geregelt ist. Dies führt einerseits dazu, daß sich Bohrschrauben von unterschiedlichen Herstellern bei oftmals sehr ähnlicher Gewindegeometrie in den vom Hersteller spezifizierten Toleranzen unterscheiden und somit auch nicht einheitlich die Toleranzangaben aus DIN EN ISO 1478 einhalten. Zum zweiten gibt es auch einige zugelassene Bohrschrauben, deren Gewindegeometrie deutlich von den in DIN EN ISO 1478 spezifizierten Gewindegeometrien abweicht (z.B. die in der Datenbank im Anhang A in der Tabelle A.2 aufgeführte Schraube Nr. 65 mit einem Nennwert des Gewindeaußendurchmessers von $6,0 \mathrm{~mm})$.

Eine Bohrschraube ist durch die Gewindegeometrie alleine weder hinsichtlicht ihres möglichen Anwendungsbereiches noch hinsichtlich der geometrischen Randbedingungen, welche die Tragfähigkeit der Verbindung bestimmen, genügend genau spezifiziert. Hierzu wäre mindestens noch eine Festlegung für die Bohrspitzengeometrie - aber auch für die mechanischen Eigenschaften und für die Kopfgeometrie - erforderlich. In DIN 7975 werden abhängig von der Blechdicke und der Zugfestigkeit der Unterkonstruktion für die meisten der in DIN EN ISO 1478 aufgeführten Gewindegeometrien die in der Unterkonstruktion durch die Vorbohrung zu erzielenden Kernlochdurchmesser angegeben. Diese Kernlochdurchmesser können für die Festlegung des Bohrspitzendurchmessers für die in der 
Leichtbauweise verwendeten Bohrschrauben jedoch höchstens als grobe Anhaltswerte dienen. Grund hierfür ist, daß die in DIN 7975 angegebenen Werte aus empirischen Untersuchungen resultieren [7], deren Randbedingungen für die Leichtbaupraxis nicht mehr zutreffen. So wurden die Untersuchungen an zweischnittigen Verbindungen mit unbeschichteten gewindeformenden Schrauben aus einsatzvergütetem Stahl mit einem Durchgangsloch im oberen Bauteil - also einem Bohrloch in diesem Bauteil, dessen Durchmesser größer ist als der Gewindeaußendurchmesser der Schraube - durchgeführt. Entgegen diesen Randbedingungen werden in der Leichtbauweise zur Gewährleistung einer größeren Korrosionsbeständigkeit vermehrt Schrauben aus nichtrostendem Stahl eingesetzt oder aber bei Verwendung von einsatzvergüteten Stahlschrauben werden diese galvanisch verzinkt. Weiterhin hat sich auch die Montage mit einem Durchgangsloch aus Wirtschaftlichkeitsgründen nicht durchgesetzt. Diese geänderten Anwendungsbedingungen führen zu einem geänderten Einschraubverhalten und somit auch zu anderen Abhängigkeiten für die erforderlichen Kernlochdurchmesser. Der wesentlichste Grund jedoch, warum die in DIN 7975 angegebenen Kernlochdurchmesser als nicht mehr zutreffend angesehen werden können, ist die Tatsache, daß sie aus der Bedingung resultieren, daß das Einschraubmoment $M_{E}$ den 0,5-fachen Wert des Mindestbruchdrehmomentes der Schraube nicht überschreitet. Für die in [27] geforderten Einschraubversuche, anhand derer eine für den Metalleichtbau zugelassene gewindeformende Schraube ihre Eignung nachweisen muß, wird dort das Einschraubmoment $M_{E}$ auf den 0,75-fachen Wert des charakteristischen Bruchdrehmomentes der Schraube begrenzt. Im Vergleich zu DIN 7975 sind daher für den Leichtbau einerseits kleinere Kernlochdurchmesser zur Erzielung des gleichen Anwendungsbereiches und andererseits ein bezüglich Blechdicke und Werkstoffestigkeit der Verbindung nach oben erweiterter Anwendungsbereich möglich.

Gewindeauslauf und Übergangsbereich vom Schaft zum Schraubenkopf, (Zone (5) im Bild 2.2):

Dieser Schraubenbereich wird nach der Montage der Verbindung durch das Bauteil I und durch eine eventuell verwendete Dichtscheibe überdeckt. Der Gewindeauslauf ist keineswegs nur ein durch die Fertigung bedingtes Übel, sondern seine Ausbildung beeinflußt die Größe des Überdrehmomentes Mü,I im Bauteil I.

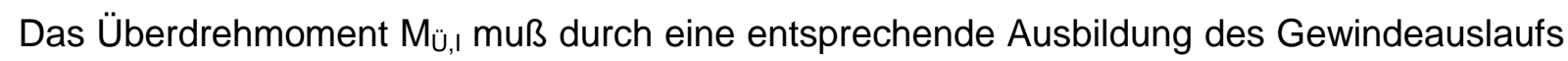

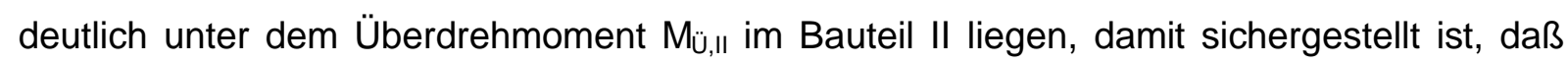
das im Bauteil II geformte Muttergewinde nicht beschädigt wird, während das im Bauteil I vorhandene Gewinde zur Erzeugung eines Durchgangsloches in diesem Bauteil zerstört wird.

Das Überdrehmoment Mü,ı nimmt zu, wenn 
- die Werkstoffestigkeit oder die Blechdicke des Bauteils I zunimmt, oder

wenn die Verzahnung zwischen dem Schraubengewinde und dem Bauteil I zunimmt.

Der kontinuierlich kleiner werdende Gewindeaußendurchmesser der Schraube im Gewindeauslauf sorgt dafür, daß im Gewindeauslauf eine im Vergleich zum vollständig ausgeformten Schraubengewinde kleinere Verzahnung mit Bauteil I entsteht.

Somit wird durch den Gewindeauslauf für Anwendungsfälle, in denen die Blechdicke und die Werkstoffestigkeit des Bauteils I nicht größer ist als die Blechdicke und die Werkstoffestigkeit des Bauteils II (Verbindungstypen 1a und 2a im Bild 2.1), ein ausreichend großer Sicherheitsabstand zwischen den Überdrehmomenten $M_{u ̈, I}$ und $M_{u ̈, I I}$ erzielt.

Für Anwendungsfälle, in denen die Blechdicke des Bauteils I größer ist als die Blechdicke des Bauteils II (Verbindungstyp 3 im Bild 2.1), besteht die Gefahr, daß das Überdrehmoment $M_{u ̈, I}$ größer wird als das Überdrehmoment $M_{u ̈, l l}$. Für diese Anwendungsfälle können die Schrauben hinter dem Gewindeauslauf mit einem Hinterschnitt versehen werden (vgl. Bild 2.8). Während des Anziehvorgangs läuft das Bauteil I dann in diesen Hinterschnitt hinein, so daß dadurch der Gewindeauslauf nur noch über eine reduzierte Höhe in das obere Bauteil eingreift, was zu einer weiteren Reduktion des Überdrehmomentes $M_{u ̈, I}$ führt.

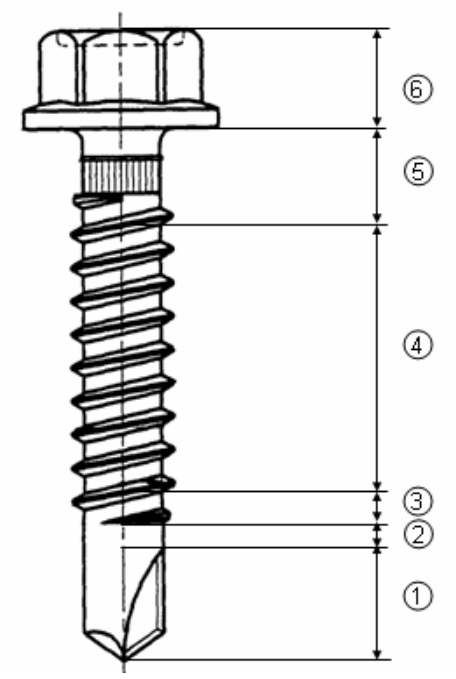

Bild 2.8: Schraube mit Hinterschnitt

\section{Schraubenkopf, (Zone (6) im Bild 2.2):}

Der Schraubenkopf dient schließlich als letzte Zone mit seiner Unterfläche als Auflage, gegen welche die zu verbindenden Bauteile während des Anziehvorgangs verspannt werden. Der Schraubenkopf bestimmt die Form des Schlüsselangriffes, die während der Montage der Verbindung die Kraftübertragung zwischen Werkzeug und Schraube gewährleistet. Die Wahl einer Kopfform unterliegt damit montagetechnischen Gesichtspunkten. Die Kopfform beeinflußt aber auch die Tragfähigkeit der Verbindung für den unter einer Zugbeanspruchung möglichen Versagensfall des Durchknöpfens des Schraubenkopfes durch das Bauteil I. An- 
dererseits prägt die Kopfform einer Schraube auch das Erscheinungsbild einer Fassade. Die Ausbildung einer möglichst steifen Kopfform zur Erzielung einer großen Durchknöpftragfähigkeit steht dabei im Gegensatz zu dem Wunsch nach einer möglichst flachen Kopfform, die den als optisch störend empfundenen Schattenwurf vermindert. Die extremen Kopfformen, die sich aus diesen gegensätzlichen Aspekten ergeben sind der Sechskantkopf mit oder ohne angeformten Bund und der Flachkopf (Bild 2.9).
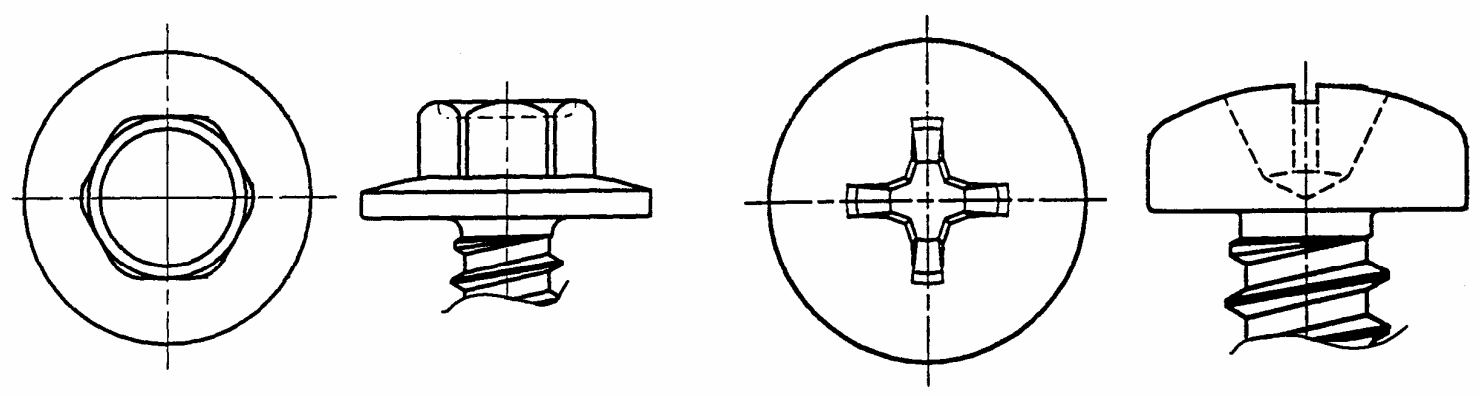

Bild 2.9: Kopfformen

Die anhand des Beispieles einer Bohrschraube zur Verbindung von direkt übereinanderliegenden Bauteilen beschriebenen wesentlichen funktionalen Zonen treten in ähnlicher Form auch bei Bohrschrauben auf, die für Verbindungen von auseinanderliegenden Bauteilen (z.B. Verbindungen zwischen einer Unterkonstruktion und dem Obergurt eines Profilblechs - Verbindungstypen $1 \mathrm{~b}$ und $2 \mathrm{~b}$ in Bild 2.1 - oder einem Sandwichelement) verwendet werden. Eine solche Bohrschraube ist in Bild 2.10 dargestellt. Der wesentliche Unterschied bei diesen Schrauben besteht darin, daß sich der Schraubenschaft, der aufgrund der zu überbrückenden Distanz natürlich sehr viel länger ist als bei einer Bohrschraube, in zwei durch einen gewindefreien Bereich getrennte Gewindezonen gliedert. Das obere Gewinde besitzt dabei häufig einen größeren Gewindeaußendurchmesser als das untere Gewinde, weil es dem oberen Blech als Stützgewinde dienen soll, wenn dieses durch eine nach unten wirkende Belastung beansprucht wird. Der zwischen den zwei Gewindezonen vorhandene gewindefreie Bereich hat die Aufgabe eine Trennung zu schaffen zwischen dem Bohrvorgang in der Unterkonstruktion und dem Gewindeformvorgang zwischen dem oberen Schraubengewinde und dem Bauteil I. Diesem Bereich kommt also eine ähnliche Funktion zu wie der Zone (2) der Bohrschraube im Bild 2.2. 


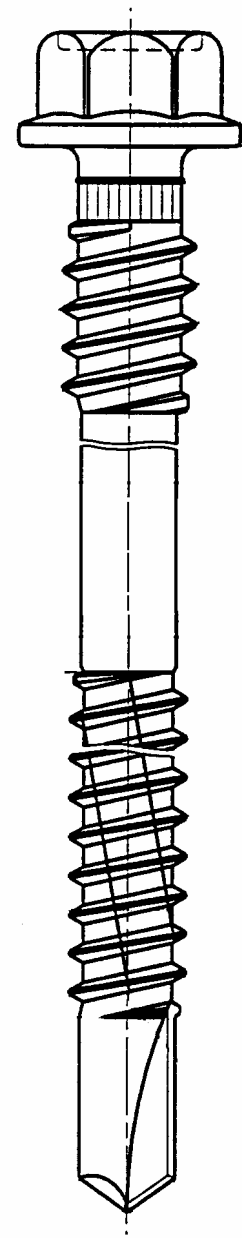

Bild 2.10: Bohrschraube für die Verbindung von auseinanderliegenden Bauteilen Die Geometrie einer gewindefurchenden Schraube unterscheidet sich von derjenigen einer Bohrschraube nur dadurch, daß bei gewindefurchenden Schrauben die Zonen (1) und (2) nicht vorhanden sind, da für gewindefurchende Schrauben das in den zu verbindenden Bauteilen erforderliche Kernloch in einem extra Arbeitschritt vorgebohrt werden muß. Die geometrische Gestaltung der restlichen Zonen (3) bis (6) ist völlig identisch. Insbesondere gelten auch für gewindefurchende Schrauben die für Bohrschrauben oben erläuterten Zusammenhänge, daß durch die Kombination aus Gewindegeometrie und gewähltem Vorbohrdurchmesser sowohl die Auszugstragfähigkeit als auch der Montagevorgang - und somit der Anwendungsbereich - einer gewindefurchenden Schraube im wesentlichen beeinflußt wird. Verglichen mit Bohrschrauben sind gewindefurchende Schrauben in ihrem Anwendungsbereich wesentlich flexibler, da für eine gewindefurchende Schraube durch eine geeignete Variation des Vorbohrdurchmessers ein hinsichtlich Blechdickenbereich und Werkstoffestigkeit der Verbindung weiter Anwendungsbereich abgedeckt werden kann. Anderseits sind gewindefurchende Schrauben aufgrund des zusätzlichen Arbeitschrittes der Erzeugung der Vorbohrung weniger wirtschaftlich als Bohrschrauben. Diese zwei Aspekte - der Vorteil eines variablen Einsatzbereiches auf der einen Seite und der Nachteil des zusätzlichen Arbeit- 
schrittes auf der anderen Seite - sind die Ursachen dafür, daß sich für die Anwendung lediglich die zwei in Bild 1.4 b) und c) dargestellten gewindefurchenden Schraubentypen durchgesetzt haben.

Dies ist zum einen eine gewindefurchende Schraube mit einer Gewindegeometrie St 6,3 nach DIN EN ISO 1478 (in der Praxis auch als Feingewinde oder Gewindeform Typ B bezeichnet). Durch eine geeignete Variation des Vorbohrdurchmessers ist diese Schraube einsetzbar für Verbindungen von Profilblechen oder Sandwichelementen auf Unterkonstruktionen aus Stahl oder Aluminium mit Bauteildicken im Bereich von $2 \mathrm{~mm}$ bis 12mm (vgl. [3], [4], DIN 18807-6 und -9).

Als zweites ist eine gewindefurchende Schraube zu nennen, deren Gewindegeometrie starke Ähnlichkeiten aufweist zu dem in DIN 7998 genormten Holzschraubengewinde (in der Praxis auch als Grobgewinde oder Gewindeform Typ A bezeichnet). Das Gewinde ist tief ausgerollt $\left(d_{g}=6,5 \mathrm{~mm}, d_{k}=4,7 \mathrm{~mm}\right)$ und besitzt im Vergleich zu den Gewindegeometrien gemäß DIN EN ISO 1478 eine größere Gewindesteigung ( $P=2,54 \mathrm{~mm})$. Der Anwendungsbereich dieser Schraube liegt in der Verbindung von Profilblechen oder Sandwichelementen auf Unterkonstruktionen aus Stahl oder Aluminium mit Bauteildicken bis zu 2mm oder aber auf Holzunterkonstruktionen (vgl. [3], [4], DIN 18807-6 und -9).

Die obigen Ausführungen verdeutlichen, daß gebräuchliche gewindeformende Schrauben in der Leichtbauweise schon aufgrund des Fehlens einer einheitlichen und bindenden Regelung für die möglichen Kombinationen zwischen der Gewindegeometrie und dem Bohrspitzendurchmesser für Bohrschrauben oder dem Durchmesser der Vorbohrung für gewindefurchende Schrauben als nicht geregelte Verbindungselemente anzusehen sind. Der Versuch, die Tragfähigkeiten von Verbindungen mit gewindeformenden Schrauben alleine über eine statistische Auswertung von Tragfähigkeitsversuchen formelmäßig zu erfassen, ohne dabei die die Tragfähigkeit bestimmenden mechanischen Hintergründe zu kennen, birgt somit immer das Risiko, daß aufgrund einer begrenzten Datenbasis nicht die gesamte Bandbreite an möglichen Randbedingungen - insbesondere die geometrischen Randbedingungen, die sich aus der Verwendung eines bestimmten Schraubentyps ergeben - erfaßt werden.

\subsubsection{Ausgangswerkstoffe und mechanische Eigenschaften}

In Abhängigkeit von den durch den Anwendungsbereich bestimmten mechanischen und korrosionstechnischen Anforderungen an die gewindeformenden Schrauben werden Schrauben aus niedriglegierten Kohlenstoffstählen, Schrauben aus nichtrostendem Stahl oder aber die sogenannten Bimetallschrauben verwendet. Bei letzteren wird die Bohrspitze und eventuell auch noch der beim Gewindeformvorgang am meisten beanspruchte Gewindebereich (Zonen (1) bis (3) im Bild 2.2) aus niedriglegiertem Kohlenstoffstahl hergestellt, während der im 
Beanspruchungszustand der Verbindung belastete Schraubenbereich (Zonen (4) bis (6) im Bild 2.2) aus nichtrostendem Stahl besteht.

Als niedriglegierte Kohlenstoffstähle werden Vergütungsstähle - z.B. Werkstoff 23B2 nach DIN EN 10263-4 - verwendet. Die aus Vergütungsstählen hergestellten Schrauben erhalten ihre mechanischen Eigenschaften aus einer geeigneten Wärmebehandlung. Die Art dieser Wärmebehandlung muß zur Gewährleistung eines einwandfreien Gewindeformvorgangs und für Bohrschrauben noch zusätzlich auch zur Gewährleistung eines einwandfreien Bohrvorgangs auf die Werkstoffestigkeit der zu verbindenden Stahlbauteile abgestimmt sein.

Einsatzvergütete Schrauben erhalten durch die Einsatzhärtung und die anschließende Anlaßbehandlung eine Randhärte von ca. 450 bis 570 HV0,3 mit einer Einsatzhärtungstiefe im Bereich von $0,05 \mathrm{~mm}$ bis $0,10 \mathrm{~mm}$, während der Kern einen vergüteten Werkstoffzustand mit besseren Zähigkeitseigenschaften und eine Kernhärte im Bereich von ca. 290 bis 400 HV10 aufweist (vgl. [28] und DIN EN ISO 7085) - in der Regel wird nach [28] ein Vergütungszustand der Festigkeitsklasse 10.9 gemäß DIN EN ISO 898-1 im Kern eingestellt.

Carbonitriert vergütete Schrauben weisen im Kern einen ähnlichen Vergütungszustand auf wie die einsatzvergüteten Schrauben, erreichen aber im Gegensatz zu diesen gemäß [28] eine Randhärte (Randabstand 0,05mm) bis zu ca. 650 HV0,3, was sich auf die Gewindeform- und Bohreigenschaften dieser Schrauben günstig auswirkt.

Die partielle Härtung im Bereich der Bohrspitze und der Gewindeformzone (Zonen (1) bis Anfang (4) im Bild 2.2) oder die partielle Anlaßbehandlung in dem Schraubenbereich, der im Beanspruchungszustand der Verbindung belastet ist (Zonen (4) bis (6) im Bild 2.2), bietet eine Möglichkeit, im erstgenannten Bereich Härtewerte bis über 800 HV0,3 [28] einzustellen, während in dem zweitgenannten durch die Verbindung belasteten Schraubenbereich ein homogener Vergütungszustand über nahezu den gesamten Querschnitt eingestellt werden kann. Letzteres mindert das Sprödbruchrisiko. Durch die partielle Wärmebehandlung carbonitriert vergüteter oder carbonitriert gehärteter Schrauben besteht die Möglichkeit, höherfeste Stahlbauteile und Leichtbauelemente (Festigkeitsklassen $\geq$ S355) mit Hilfe von gewindeformenden Schrauben zu verbinden.

Zur Verbesserung ihrer Korrosionsbeständigkeit werden die Schrauben aus niedriglegierten Kohlenstoffstählen galvanisch verzinkt - [4] schreibt eine Zinkschichtdicke von mindestens $8 \mu \mathrm{m}$ vor. Die galvanische Verzinkung bringt zusätzlich den positiven Effekt mit sich, daß das während des Gewindeformvorgangs auftretende maximale Einschraubmoment $M_{E}$ reduziert wird. Aufgrund der trotz der galvanischen Verzinkung geringen Korrosionsbeständigkeit dieser Schrauben, ist ihr Anwendungsbereich gemäß [4] beschränkt auf Verbindungen von Stahlbauteilen, die nicht überwiegend der Außenluft ausgesetzt sind wie z.B. die Verbindungen mit der Innenschale in zweischaligen Konstruktionen (Verbindungstypen 2a, 2b und 3 im 
Bild 2.1) oder Verbindungen zwischen Profilblechen und Unterkonstruktionen in einem einschaligen Dachaufbau mit oberseitiger Wärmedämmung.

Zur Verbesserung der Korrosionsbeständigkeit der Verbindungen werden Schrauben verwendet, deren in der Verbindung liegender und durch die Verbindung belasteter Bereich aus nichtrostendem austenitischem Stahl (z.B. Werkstoffnummer 1.4301, 1.4541, 1.4401 oder 1.4571 nach DIN EN 10263-5 oder DIN EN 17440) besteht. Das sind entweder Schrauben, die ganz aus nichtrostendem Stahl bestehen, oder die oben erwähnten Bimetallschrauben. Diese beiden Schraubenarten sind gemäß [3], [4] und DIN 18807-9 aufgrund ihrer Korrosionsbeständigkeit auch für Verbindungen geeignet, die überwiegend der Außenluft ausgesetzt sind. Aufgrund der in DIN 18807-9 geforderten Anpassung der Korrosionsbeständigkeit der Verbindungselemente an die Korrosionsbeständigkeit der zu verbindenden Bauteile werden für Verbindungen von Profilblechen aus Aluminium ausschließlich solche Schrauben verwendet.

Da austenitischer Stahl nicht gehärtet werden kann, erhält er seine mechanischen Eigenschaften ausschließlich durch die Kaltverfestigung, die sich je nach Werkstoff während des Produktionsprozesses durch eine geeignete Wahl der fertigungstechnischen Parameter ergibt. Dabei sind für Schrauben aus nichtrostenden austenitischen Stählen gemäß [28] auf den Oberflächen des Gewindes Härten bis zu maximal 500 HV0,3 zu erreichen und im Schraubenkern bleiben nach [28] und DIN EN ISO 3506-4 Härten von ca. 200 HV0,3 bis 300 HV0,3 erhalten. Da die Oberflächenhärte von bestenfalls 500 HV0,3 nicht ausreicht, um den Bohrvorgang und eventuell auch den Gewindeformvorgang bei Verbindungen von Stahlbauteilen der Festigkeitklasse S235 prozeßsicher zu gewährleisten, werden Bohrschrauben, die ganz aus austenitischem Stahl bestehen, nur für Verbindungen eingesetzt, bei denen die beiden zu verbindenden Bauteile aus Aluminium bestehen. Für die Verbindungen von Profilblechen aus Stahl oder Aluminium mit Stahlbauteilen werden Bimetallschrauben verwendet. Für die Ausführung von Bimetallschrauben haben sich zwei Varianten durchgesetzt. Dies ist zum einen die in Bild 2.4 dargestellte Bohrschraube, deren Bohrspitze aus einem gehärteten Stahlplättchen besteht, welches im Schraubenkern durch Verpressen verankert wird. Zum zweiten ist es die im Bild 2.2 dargestellte Bohrschraube, bei der die aus niedriglegiertem Kohlenstoffstahl bestehende Funktionsspitze, die den Bereich von Bohrspitze bis hin zu der beim Gewindeformvorgang am meisten beanspruchten Gewindezone (Zonen (1) bis Anfang (4) im Bild 2.2) umfaßt, angeschweißt ist und abschließend induktiv gehärtet wird. Die zweite Variante mit angeschweißter Funktionsspitze hat gegenüber der erstgenannten Variante mit einem Bohrplättchen den Vorteil, daß sie aufgrund der gehärteten Gewindeformzone bessere Gewindeformeigenschaften aufweist. Andererseits hat diese zweite Variante den Nachteil, daß gegenüber der Ausführung mit Bohrplättchen größere Schraubenlängen erforderlich sind, da sowohl die Funktionsspitze selbst als auch eine noch 
zu berücksichtigende Wärmeeinflußzone im nichtrostenden Stahlbereich unterhalb der Verbindung überstehen müssen, um ein sprödes Versagen der Verbindung im Beanspruchungszustand zu vermeiden und die Korrosionsbeständigkeit sicherzustellen.

Die Eignung einer Schraube für den Einsatz in einem definierten Anwendungsbereich muß nach [3] und [4] anhand von Einschraubversuchen nachgewiesen werden, die in [27] beschrieben sind. Dieser Nachweis gilt gemäß [27] als erbracht, wenn beim Eindrehen der Schrauben in Bauteile, die den bezüglich Blechdicke und Werkstoffestigkeit für die Schrauben definierten oberen Anwendungsbereich abdecken, das maximal auftretende Gewindeformmoment $M_{E}$ den 0,75-fachen Wert des Torsionsbruchmomentes der Schrauben nicht übersteigt und wenn gleichzeitig der Gewindeabrieb im eingeschraubten Gewindebereich nicht größer ist als $0,1 \mathrm{~mm}$. Die Einschraubversuche können durch den Nachweis über das Einhalten von mechanischen Kennwerten wie etwa Rand- und Kernhärten der Schrauben oder aus Zugversuchen ermittelten Werkstoffkennwerten der Schrauben nicht ersetzt werden, da die Bohr- und Gewindeformeigenschaften einer Schraube von ihren mechanischen Kennwerten aber zusätzlich auch noch im wesentlichen von der Schraubengeometrie bestimmt werden.

Die in [27] ebenfalls geforderten Zugversuche an der ganzen Schraube (Bild 2.11) dienen in erster Linie der Bestimmung und Überprüfung der Zugbruchtragfähigkeit der Schraube, die eine Obergrenze für die Beanspruchbarkeit einer zugbeanspruchten Verbindung darstellt.

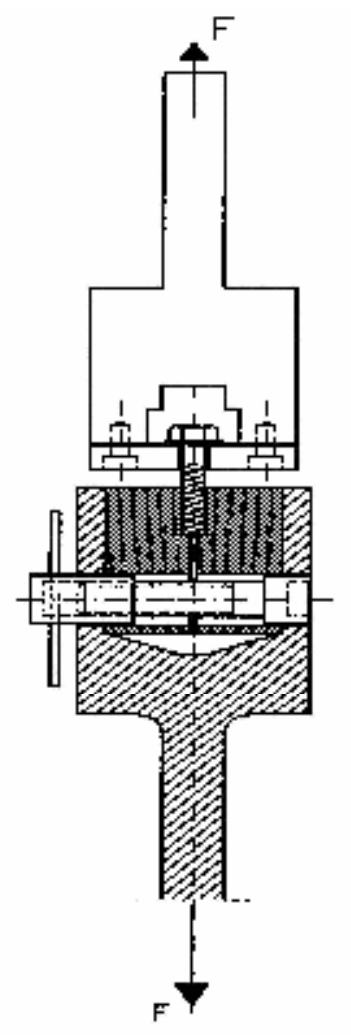

Bild 2.11: Zugversuche an der ganzen Schraube 
Die Zugversuche an der ganzen Schraube können zur Beschreibung des Werkstoffverhaltens der Schraube nur bedingt herangezogen werden.

Grund hierfür ist, daß die Zugbruchtragfähigkeit einer Schraube auch durch die Kerbwirkung der Gewindegeometrie beeinflußt wird. Für Schrauben, deren Gewinde sich über die gesamte Schaftlänge erstreckt - dies ist der Fall für gewindeformende Schrauben, die für die Verbindung von direkt übereinanderliegenden Blechen eingesetzt werden (Bild 2.2) - ist der Kernquerschnitt des Schraubengewindes für das Zugbruchversagen maßgebend. In diesem Querschnitt wird aufgrund der Kerbwirkung des Gewindes die Querkontraktion behindert. Es entsteht ein mehrachsiger gleichsinniger Spannungszustand, der zu einer Steigerung der Zugbruchtragfähigkeit führt [26], [29]. Die Zugbruchtragfähigkeit im Gewindebereich wird deshalb durch einen Bezug auf den Kernquerschnitt und die an einer ungekerbten Zugprobe der Schraube ermittelten Zugfestigkeit unterschätzt. Umgekehrt ausgedrückt wird die Zugfestigkeit des Werkstoffes überschätzt, wenn die über Zugversuche an der ganzen Schraube ermittelte Zugbruchtragfähigkeit auf den Kernquerschnitt der Schraube bezogen wird. Für Schrauben, die einen gewindefreien Schaftbereich besitzen - dies ist häufig für Schrauben der Fall, die für die Obergurtbefestigung von Profilblechen oder die Befestigung von Sandwichelementen verwendet werden (Bild 2.10) - kann der für das Zugbruchversagen maßgebende Querschnitt nur dann zuverlässig angegeben werden, wenn der Durchmesser des gewindefreien Schafts nicht größer ist als der Kerndurchmesser des Schraubengewindes. Für diesen Fall wird für das Zugbruchversagen aufgrund der Kerbwirkung des Gewindes der gewindefreie Schaftquerschnitt mit dem kleinsten Durchmesser maßgebend. Für alle anderen Fälle kann entweder der gewindefreie Schaft oder aber auch das Schraubengewinde für das Zugbruchversagen maßgebend werden. Das Schraubengewinde wird dabei maßgebend, wenn die Kerbwirkung des Gewindes nicht ausreicht, um eine im Vergleich zum gewindefreien Schaft reduzierte Querschnittsfläche des Gewindekerns zu kompensieren. Zur Abschätzung der festigkeitssteigernden Wirkung des Gewindes von gewindeformenden Schrauben können die aus dem Schriftum [26], [29], [30] bekannten Zusammenhänge für das metrische Regelgewinde herangezogen werden. Nach [26] ergibt sich aus der Kerbwirkung für ein metrisches Regelgewinde eine um ca. 10\% größere Zugfestigkeit gegenüber einem glatten Stab mit einem Querschnitt, der dem Kernquerschnitt des metrischen Regelgewindes entspricht. Da das Verhältnis der Gewindesteigung zum Gewindeaußendurchmesser mit 0,20 bis 0,39 bei gewindeformenden Schrauben deutlich größer ist als mit 0,10 bis 0,18 bei Schrauben mit metrischen Regelgewinden, ist die Kerbwirkung des Gewindes von gewindeformenden Schrauben geringer als bei metrischen Schrauben. Für metrische Schrauben wird die Kerbwirkung des Gewindes durch die Einführung eines fiktiven Spannungsquerschnittes $A_{s p}$ berücksichtigt. Dieser Spannungsquerschnitt ist für das metrische Regelgewinde ca. 10\% größer als der Kernquerschnitt des Gewindebereichs. Die Zugbruch- 
tragfähigkeit im Gewindebereich von metrischen Schrauben wird durch einen Bezug auf den Spannungsquerschnitt und die an einer ungekerbten Zugprobe der Schraube ermittelten Zugfestigkeit ermittelt.

Zusätzlich zu der Kerbwirkung des Schraubengewindes kann auch die Kopfgeometrie der Schraube einen Einfluß auf die Zugbruchtragfähigkeit besitzen. Für zugbeanspruchte Schrauben, bei denen sich der Schlüsselangriff innerhalb des Schraubenkopfes befindet (z. B. Schrauben mit einem Flachkopf und kreuzschlitzförmigen Schlüsselangriff), kann beispielsweise der Versagensfall auftreten, daß der durch den Schlüsselangriff geschwächte Schraubenkopf unter der Zugbelastung vom Kernquerschnitt abgeschert wird [31].

Da die Festigkeit der fertigen Schraube, wie oben verdeutlicht wurde, von ihren mechanischen Werkstoffeigenschaften, ihrer Kopf- und Schaftgeometrie sowie der Kerbwirkung des Gewindes abhängt, werden gemäß [27] und im Rahmen des Zulassungsverfahrens zu [3] und [4] auch zur Bestimmung des Torsionsbruchmomentes der Schrauben und der Scherbruchtragfähigkeit des in einer querkraftbeanspruchten Verbindung liegenden Gewindebereichs der Schrauben Tragfähigkeitsversuche an der ganzen Schraube durchgeführt (Bilder 2.12 und 2.13).

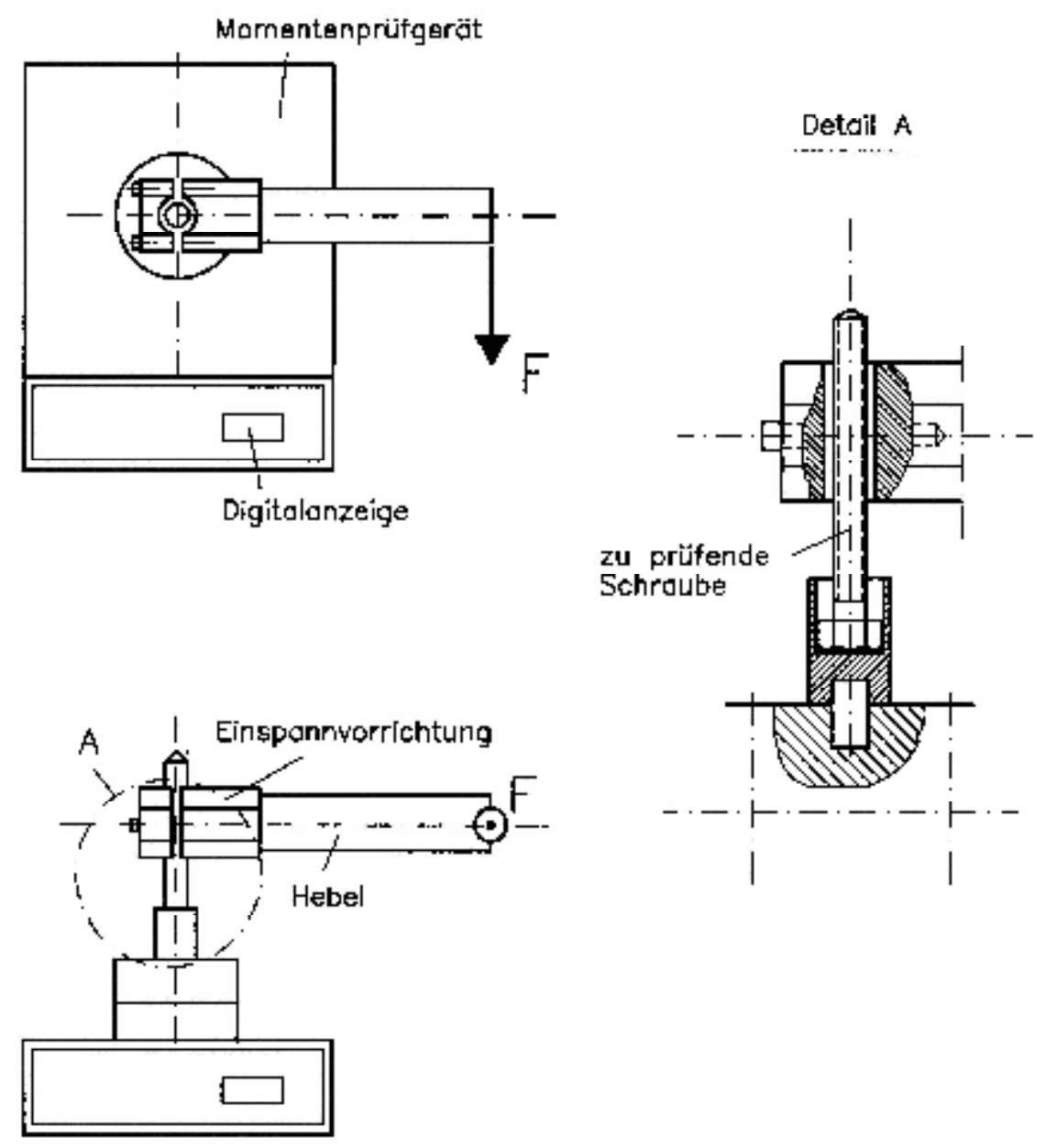

Bild 2.12: Torsionsbruchversuche an der ganzen Schraube 

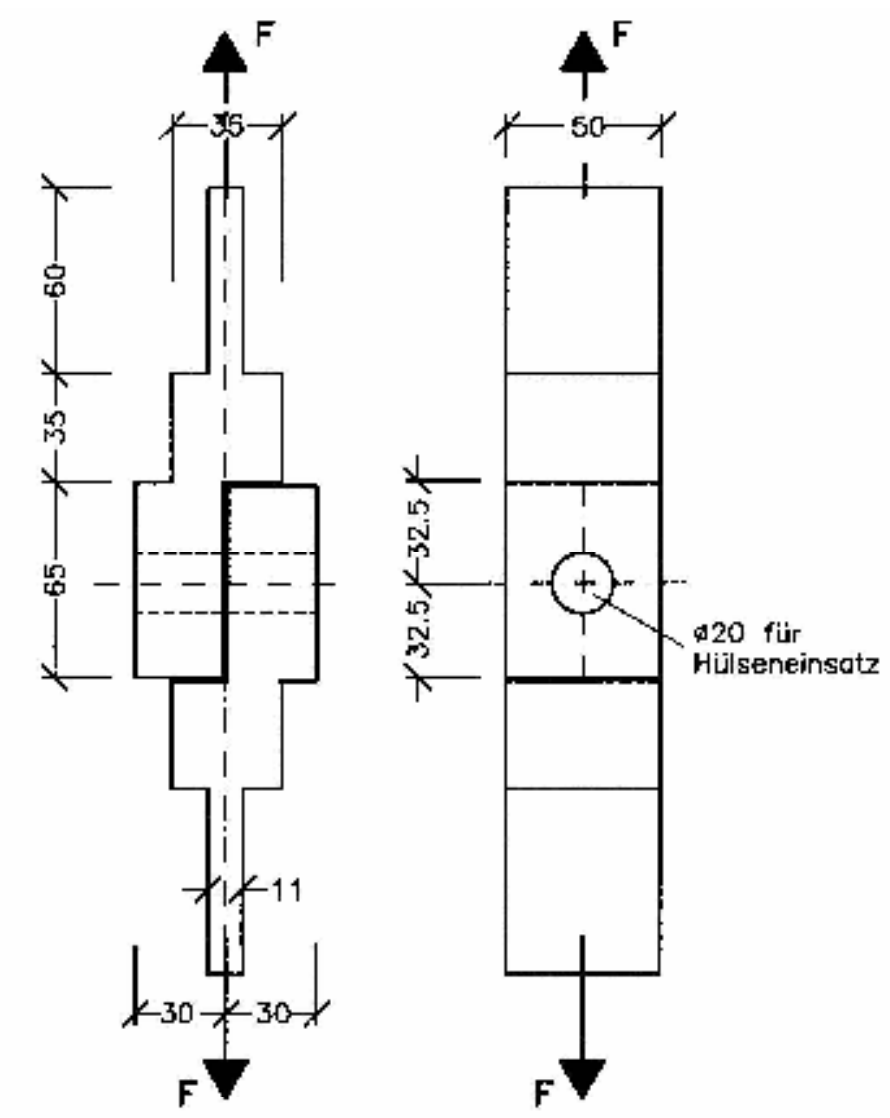

Hülsen:

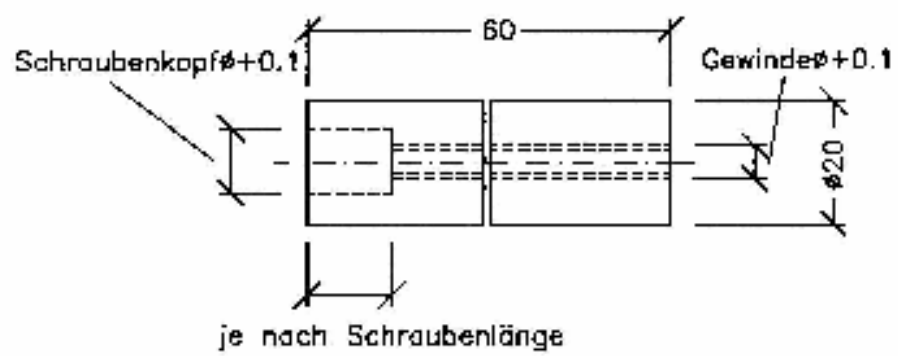

Bild 2.13: Scherbruchversuche an der ganzen Schraube

\subsection{Versuche zur Bestimmung der Auszugstragfähigkeit}

Zur experimentellen Ermittlung der Auszugstragfähigkeit der in Bild 2.1 und Tabelle 2.1 beschriebenen Verbindungen wird der gängigen Zulassungspraxis zu [3] und [4] entsprechend und in Anlehnung an [5], Entwurf DIN 18807-4 und DIN 18807-7 die in Bild 2.14 dargestellte Versuchsanordnung verwendet. 


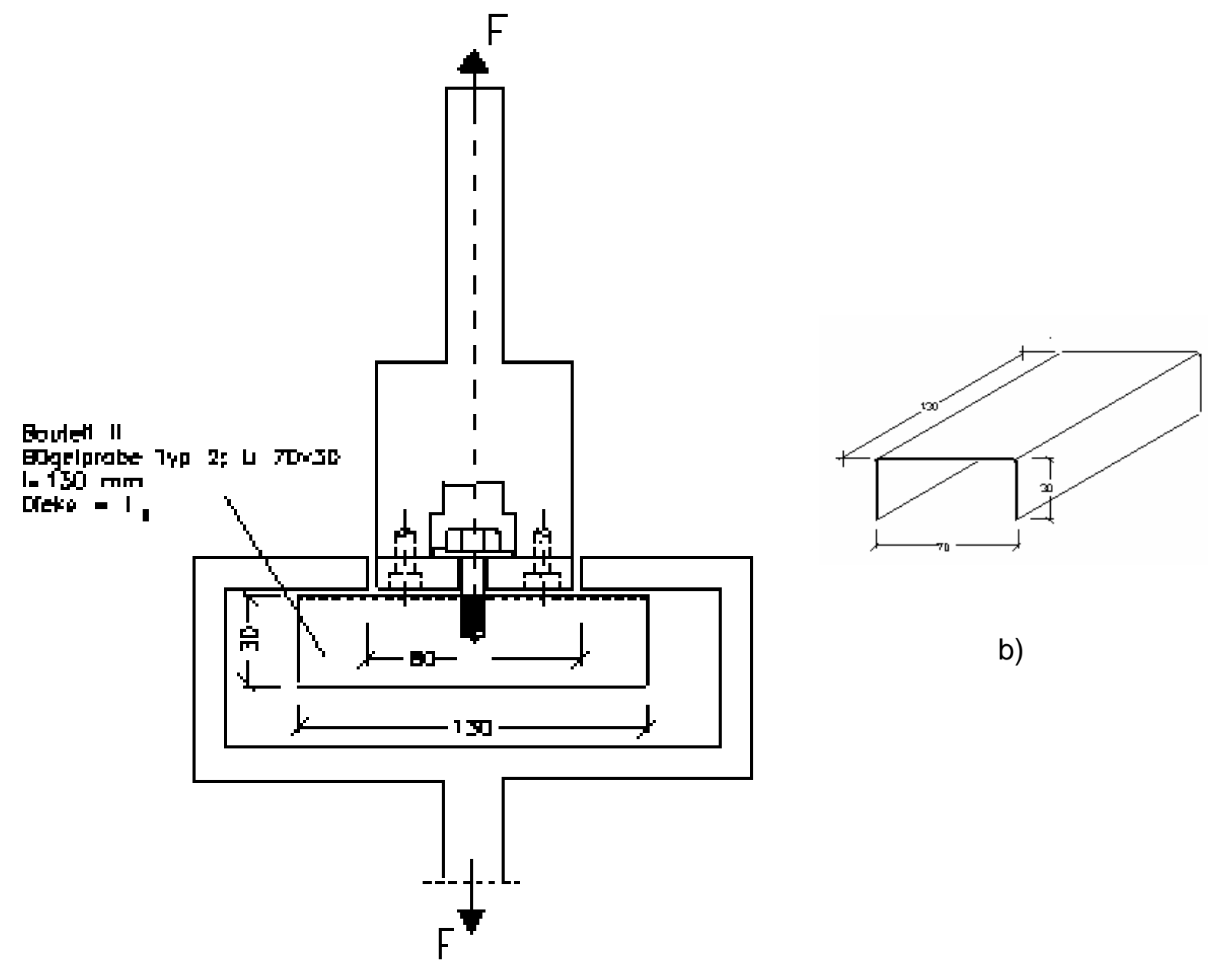

a)

Bild 2.14: a) Versuchsanordnung zur Bestimmung der Auszugstragfähigkeit, b) Standardisierte Probenform der Unterkonstruktion für $\mathrm{t}<6 \mathrm{~mm}$

Die in der Versuchsanordnung verwendete Probenform für die Unterkonstruktion muß bezüglich ihrer Blechdicke und ihrer Werkstoffeigenschaften der realen Unterkonstruktion entsprechen. Die Querschnittsform der Proben (in der Regel U 70mmx30mm für Bauteildicken der Unterkonstruktion $t_{\|}<6 \mathrm{~mm}$ und ansonsten ein Flachblech mit einer Breite $b=60 \mathrm{~mm}$ ) sowie die Stützweite (80mm) sind in [5], Entwurf DIN 18807-4 und DIN 18807-7 festgelegt, weil durch die Versuche lediglich die lokale Auszugstragfähigkeit der Verbindungsstelle untersucht werden soll und nicht etwa das globale Tragverhalten oder Verformungsverhalten der Unterkonstruktion. Die Probenform ist daher so festgelegt worden, daß ein Biegeversagen der Unterkonstruktion, die im Versuch als zweiseitig gelagerte Platte wirkt, nicht auftritt.

Da sowohl die in der Datenbank im Kapitel 3 dieser Arbeit aufgeführten Versuche als auch die im Kapitel 4 durchgeführten FE-Untersuchungen diese Versuchsanordnung zugrunde legen, kann die im Rahmen dieser Arbeit entwickelte Beschreibung der Auszugstragfähigkeit als Ersatz für diese Tragfähigkeitsversuche angesehen werden. Die entsprechend dieser Versuchsanordnung getroffene Annahme, daß die Auszugstragfähigkeit der Schraube nicht durch das Bauteil I beeinflußt wird, wird also auch bei den Untersuchungen dieser Arbeit 
getroffen. Die Gültigkeit dieser Annahme wird durch die nachfolgenden Ausführungen bewertet und eingegrenzt:

Die Auszugstragfähigkeit der Schraube wird durch die Größe der Verzahnung zwischen dem Schraubengewinde und dem Muttergewinde in der Unterkonstruktion beeinflußt. Daher muß die durch das Eindrehen und Positionieren der Schraube in der Unterkonstruktion erzielte Verzahnung zwischen Schraube und Unterkonstruktion im Versuch den realen Anwendungsbedingungen entsprechen. Diese Anwendungsbedingungen sind aufgrund der Abstimmung der Schraubengeometrie auf einen genau definierten Anwendungsbereich in der Regel dadurch gegeben, daß die Unterkonstruktion nach der Montage der Verbindung im Bereich der vollständig ausgeformten Gewindezone der Schraube anliegt (Zone (4) im Bild 2.2, Abschnitt 2.2.1). Dies wird als Voraussetzung sowohl bei den Versuchen gemäß Bild 2.14 als auch im Rahmen dieser Arbeit unterstellt.

Die in der realen Anwendung durch die Schraubenmontage erzielte Vorspannung der Verbindung darf keinen Einfluß - oder zumindest keinen negativen Einfluß - auf die Auszugstragfähigkeit der Verbindung haben. Da die Vorspannung einer zugbeanspruchten Verbindung - ähnlich wie bei vorgespannten Stirnplattenverbindungen mit metrischen Schrauben [30], [32] - [34] - eine Erhöhung der Steifigkeit der Verbindung unter Gebrauchslasten sowie eine Erhöhung ihrer Tragfähigkeit unter einer wiederholten Beanspruchung zu Folge hat, jedoch keine Auswirkungen auf die statische Tragfähigkeit der Verbindung mit sich bringt, kann diese Bedingung als erfült betrachtet werden.

Die Versuchsanordnung gemäß Bild 2.14 berücksichtigt nicht die Vergrößerung der Beanspruchung für die Verbindung, die aus eventuell auftretenden Kontaktkräften zwischen den Bauteilen I und II resultiert (Bild 2.15). Mit Kontaktkräften ist immer dann zu rechnen, wenn die Verbindungsstelle in mindestens einem der beiden Bauteile I oder II exzentrisch angeordnet ist und wenn gleichzeitig dieses Bauteil - bedingt durch eine kleine Blechdikke - eine kleine Steifigkeit aufweist, die zu Relativverdrehungen zwischen den Bauteilen I und II führt, bevor die Verbindung ihre Tragfähigkeit erreicht. Dies hat zur Folge, daß gemäß [4], Entwurf DIN 18807-4 und DIN 18807-7 für diejenigen Verbindungstypen, in denen ein Bauteil eine große Blechdicke $t \geq 5 \mathrm{~mm}$ aufweist und gleichzeitig die Positionierung der Schraube im Profilquerschnitt des durch ein dünnwandiges Profilblech $(\mathrm{t} \leq 1,50 \mathrm{~mm})$ gebildeten zweiten Bauteils zentrisch erfolgt, auch dann keine Kontaktkräfte auftreten, wenn die Verbindungsstelle exzentrisch in dem ersten Bauteil liegt, weil die Steifigkeit dieses Bauteils in diesen Fällen ausreicht, um eine Relativverdrehung zwischen den beiden Bauteilen bis zum Erreichen der Tragfähigkeit der Verbindung zu verhindern. Für alle anderen Fälle wirkt sich der Einfluß von Kontaktkräften lediglich auf die Durchknöpftragfähigkeit der Verbindung ungünstig aus, weil die aufgrund der Kontaktkräfte sich einstellende Verdrehung der Verbindung dazu führt, daß der Schraubenkopf nicht 
mehr über seine ganze Auflagefläche am Bauteil I anliegt, was zusätzlich zu der durch die Kontaktkräften erhöhten Beanspruchung noch eine Reduktion der Durchknöpftragfähigkeit bewirkt [5], [6], [35]. Im Gegensatz dazu kommt es aufgrund einer Verdrehung der Verbindung zu einer Verklemmung der Schraube in der Unterkonstruktion, was zu einer Vergrößerung der Auszugstragfähigkeit führt [5], [6] und somit dem negativen Effekt der durch die Kontaktkräfte vergrößerten Beanspruchung entgegenwirkt. Die im Bild 2.14 dargestellte Versuchsanordnung liefert somit gemäß [5] und [6] auch für Verbindungen, bei denen Kontaktkräfte auftreten, eine auf der sicheren Seite liegende Abschätzung für die Auszugstragfähigkeit. Zur Festlegung der Durchknöpfbeanspruchbarkeit kann ein bei der versuchsgestützten Bestimmung dieser Beanspruchbarkeit nicht berücksichtigter Einfluß von Kontaktkräften über Abminderungsbeiwerte erfaßt werden, die in [4], Entwurf DIN 18807-4 und DIN 18807-7 angegeben werden.

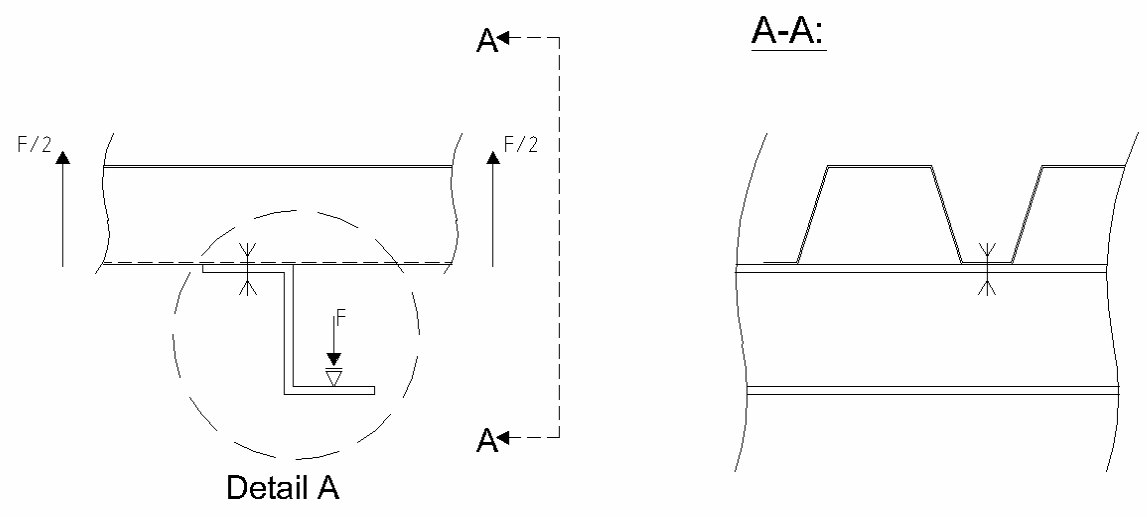

Detail A:

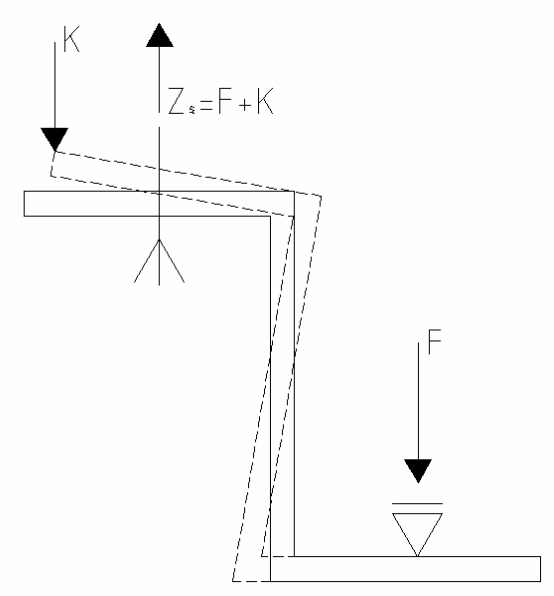

Bild 2.15: Kontaktkräfte zwischen Bauteil I und Bauteil II 


\section{Versuchsdatenbank}

Im Anhang A ist in den Tabellen A.1 und A.2 eine Datenbank zusammengestellt, mit der 230 unterschiedliche Serien von Auszugversuchen mit gewindeformenden Schrauben und Unterkonstruktionen aus Stahl und Aluminium beschrieben werden. Die Auszugversuche wurden an der Versuchsanstalt für Stahl, Holz und Steine im Rahmen des Zulassungsverfahrens zu [3] und [4] oder für Verbindungen im Anwendungsbereich von DIN 18807-7 durchgeführt. Zur Beschreibung der geometrischen und werkstoffspezifischen Eigenschaften der in den Auszugversuchen verwendeten Schrauben wurden teilweise auch Informationen verwendet, die an der Versuchsanstalt für Stahl, Holz und Steine aufgrund ihrer Tätigkeit als fremdüberwachende Stelle für die Hersteller von gewindeformenden Schrauben vorliegen.

In der Tabelle A.1 sind für die einzelnen Serien von Auszugversuchen, folgende Informationen spaltenweise erfaßt:

- $\quad$ In der Spalte 1 ist die Quelle angegeben, in der die jeweilige Versuchsserie dokumentiert ist und aus der die Informationen der nachfolgenden Spalten entnommen wurden. Bei den Quellen handelt es sich in allen Fällen um unveröffentlichte Berichte [49] der Versuchsanstalt für Stahl Holz und Steine.

- $\quad$ In der Spalte 2 wurde für jede neue Versuchsserie eine laufende Nummer vergeben.

- Mit Hilfe der Spalten 3 bis 8 werden die Randbedingungen beschrieben, die den unterschiedlichen Versuchsserien zugrunde liegen. Der verwendete Schraubentyp wird durch die in der Spalte 3 angegebene Nummer gekennzeichnet. Die geometrischen und werkstoffspezifischen Eigenschaften des jeweiligen Schraubentyps werden durch einen Bezug auf die für inn vergebene Nummer in der Tabelle A.2 beschrieben. In der Spalte 4 wurde für unterschiedliche Werkstoffchargen der Unterkonstruktion ebenfalls eine laufende Nummer vergeben. In der Spalte 5 ist die Nennblechdicke $t_{N}$ der Unterkonstruktion aufgeführt, und aus der Spalte 6 wird ersichtlich, ob es sich um eine Stahlunterkonstruktion (S) oder eine Unterkonstruktion aus einer Aluminiumlegierung (A) handelt. In der Spalte 7 ist die Querschnittsform der für die Unterkonstruktion verwendeten Probekörper angegeben. Als Querschnittsformen wurden in den erfaßten Versuchen entweder U-Profile (in der Tabelle bezeichnet durch: „U-Gurtbreite in mm-Schenkelhöhe in mm“) oder Flachbleche (in der Tabelle bezeichnet durch: „FL-Breite in $\mathrm{mm}^{\prime}$ ) verwendet. Sofern in einer Versuchsserie eine gewindefurchende Schraube geprüft wurde, ist in der Spalte 8 der Bohrdurchmesser angegeben, mit dem die Unterkonstruktion zur Erzeugung des erforderlichen Kernloches vor der Montage der Schraube vorgebohrt wurde. 
- In den Spalten 9 bis 16 werden die Ergebnisse der Auszugversuche einer Versuchsserie zusammengefaßt. In der Spalte 9 ist die Anzahl $n_{A z V}$ der Einzelversuche der Versuchsserie angegeben. In den Spalten 10 und 11 werden der Mittelwert und die Standardabweichung der an den für die Unterkonstruktion verwendeten Probekörpern gemessenen Kernblechdicken $t_{k}$ angegeben. In den Spalten 12 bis 16 werden der Mittelwert, das Minimum, das Maximum sowie die Standardabweichung der in den Einzelversuchen erreichten Versagenslasten und die Versagensart angegeben. Als Versagensart wird dabei zwischen dem Zugbruch der Schraube (ZB) und dem Auszug der Schraube aus der Unterkonstruktion ( $A Z$ ) unterschieden. Für das Auszugversagen liegen keine Informationen darüber vor, ob dieses Versagen durch ein Versagen des in der Unterkonstruktion geformten Muttergewindes, durch ein Versagen des Schraubengewindes oder durch eine Kombination dieser beiden Versagensmechanismen stattgefunden hat.

- In den Spalten 17 bis 20 werden die Ergebnisse aus einaxialen Zugversuchen zusammengefaßt, die zur Ermittlung der Werkstoffkennwerte der in den Auszugversuchen verwendeten Unterkonstruktionen durchgeführt wurden. In der Spalte 17 ist die Anzahl $n_{z v}$ dieser Zugversuche angegeben, und die folgenden Spalten geben die aus den Zugversuchen ermittelten Mittelwerte der 0,2\%-Dehngrenze $\left(R_{P 0,2}\right)$ und der Zugfestigkeit $\left(R_{m}\right)$ sowie die Standardabweichung für die Zugfestigkeiten $\left(\sigma_{\mathrm{Rm}}\right)$ wieder.

In der Tabelle A.2 wurden zur Beschreibung der in den Auszugversuchen verwendeten Schrauben folgende Informationen erfaßt:

- In der Spalte 1 wird durch die Angabe der laufenden Nummern für die Schrauben ein Bezug zur Tabelle A.1 hergestellt.

- In der Spalte 2 wird der Ausgangswerkstoff der Schrauben („C“ steht für einen niedriglegierten Kohlenstoffstahl und „NR“ steht für einen nichtrostenden, austenitischen Stahl) angegeben. Diese Angabe bezieht sich nur auf den durch die Verbindung belasteten Schraubenbereich (Zonen (4) bis (6) im Bild 2.2, Abschnitt 2.2.1). Über die Bezeichnung „NR" werden daher sowohl Schrauben, die ganz aus nichtrostendem Stahl hergestellt werden, als auch die sogenannten Bimetallschrauben erfaßt.

- In den Spalten 3 bis 12 wurden die Toleranzangaben der Hersteller für diejenigen geometrischen Kennwerte der Schrauben erfaßt, die möglicherweise einen Einfluß auf die Auszugstragfähigkeit haben können. In den Spalten 3 bis 10 wurden die Toleranzangaben für den Gewindeaußendurchmesser $d_{g}$, den Kerndurchmesser $d_{k}$, die Steigung $P$ sowie den Flankenwinkel $\alpha$ des in die Unterkonstruktion eingreifenden Schraubengewindes (Bild 2.7, Abschnitt 2.2.1) angegeben. Maßangaben für die Abflachung c oder die Basis b des Gewindeprofils werden von den Schraubenherstellern in der Regel nicht gemacht. Grund hierfür ist, daß diese Maße nicht planmäßig für die Fertigung vorgegeben 
werden, sondern sie werden durch den Produktionsprozeß und durch die Einhaltung der Toleranzangaben für die Maße $d_{g}, d_{k}$ und a implizit gesteuert und kontrolliert. Für Bohrschrauben wurden in den Spalten 11 und 12 die Toleranzangaben für den Bohrspitzendurchmesser $d_{B s}$ aufgenommen. Für gewindefurchende Schrauben wurde in diese Spalten die Bezeichnung „GF“ eingetragen. Sofern in den Spalten 3 bis 12 für eine geometrische Kenngröße die angegebenen Max.- und Min.-Werte übereinstimmen - dies ist überwiegend für die Gewindesteigung $P$ und den Flankenwinkel a der Fall - bedeutet dies, daß es sich um einen Nennwert für die jeweilige geometrische Eigenschaft handelt, für die keine Toleranzangaben vorliegen.

- In den Spalten 13 bis 25 sind die Ergebnisse von Maßprüfungen an den Schrauben sowie die Ergebnisse von Prüfungen zur Bestimmung der Zugbruchtragfähigkeiten der Schrauben erfaßt. In der Spalte 13 sind die Quellen angegeben, aus denen diese Ergebnisse entnommen wurden. Als eine Quelle wurden die in der Tabelle A.1 bereits aufgeführten Versuchsberichte herangezogen. Darüber hinaus wurden auch Versuche herangezogen, die im Rahmen der Fremdüberwachung durchgeführt wurden. Die Ergebnisse, die aus einem Versuchsbericht stammen, sind in der Spalte 13 durch die Angabe der entsprechenden Berichtnummer gekennzeichnet. Sofern zusätzlich zu der Angabe der Berichtnummer auch noch die in der Tabelle A.1 vergebene laufende Nummer für eine Versuchsserie aufgeführt ist, bedeutet dies, daß die Zugbruchlasten aus dieser Versuchsserie entnommen wurden, da in der entsprechenden Serie das Versagen durch einen Zugbruch der Schrauben stattgefunden hat. Die Ergebnisse, die aus der Fremdüberwachung resultieren, sind in der Spalte 13 durch die Bezeichnung „FÜ“ gekennzeichnet. Als Ergebnisse der Maßprüfungen sind in den Spalten 14 bis 22 die Anzahl der geprüften Schrauben sowie die Mittelwerte und die Standardabweichungen für die Maße $d_{g}, d_{k}, P$ und $d_{B s}$ angegeben. Meßergebinsse für die Gewindeabflachung $c$, die Basis $b$ oder den Flankenwinkel a lagen für keine Schraube vor, da in [27] eine Überprüfung dieser Maße nicht gefordert wird. Als Ergebnisse der Zugbruchversuche sind in den Spalten 23 bis 25 die Anzahl der Prüfungen sowie die Mittelwerte und die Standardabweichungen der Versagenslasten angegeben. Für die erfaßten Zugbruchversuche kann darüber hinaus festgestellt werden, daß in keinem Fall das Versagen durch ein Abscheren des durch einen innenliegenden Schlüsselangriff geschwächten Schraubenkopfes auftrat. Die aus der Fremdüberwachung entnommenen Ergebnisse der Maßprüfungen und der Zugbruchversuche, die in der gleichen Zeile für einen Schraubentyp erfaßt sind, sind mit Schrauben aus ein und derselben Charge bestimmt worden. Für die aus den Versuchsberichten entnommenen Ergebnisse kann dies nicht zuverlässig bestätigt werden, weil bei der versuchsgestützten Bestimmung der Tragfähigkeiten von geschraubten Verbindungen (Zulassungsversuche) in der Regel eine Vielzahl von Versuchen - Querzugver- 
suche, Durchknöpfversuche, Auszugversuche sowie Tragfähigkeitsversuche an den Schrauben selbst - durchgeführt werden müssen, und daher für einen bestimmten Schraubentyp sehr viele Schrauben benötigt werden, die dann aus unterschiedlichen Fertigungschargen stammen können. Eine Zuordnung von eventuell unterschiedlichen Fertigungschargen zu unterschiedlichen Versuchsanordnungen ist jedoch aus den in den Versuchsberichten vorliegenden Informationen in der Regel nicht möglich.

- Der Spalte 26 ist eine Abschätzung für die Zugfestigkeit des Schraubenkernquerschnittes im Gewindebereich $R_{m, s G}$ zu entnehmen. Für diese Abschätzung wurde der Mittelwert der Zugbruchlast aus Spalte 24 durch den Kernquerschnitt dividiert. Zur Berechnung des Kernquerschnittes wurde der in den Maßprüfungen festgestellte mittlere Kerndurchmesser $d_{k}$ des Schraubengewindes (Spalte 17) herangezogen. Sofern für einen Schraubentyp in einer der in Spalte 13 angegebenen Quellen keine Maßprüfungen dokumentiert waren, aber Zugbruchlasten aus dieser Quelle vorlagen, wurde für den Kerndurchmesser der Mittelwert aus den in den Spalten 5 und 6 aufgeführten Toleranzangaben eingesetzt. Bei den in der Datenbank aufgeführten Schrauben handelt es sich um Schrauben, die entweder ein Gewinde besitzen, das sich über die gesamte Schaftlänge erstreckt (Bild 2.2, Abschnitt 2.2.1), oder aber um Schrauben mit einem gewindefreien Schaftbereich (Bild 2.10, Abschnitt 2.2.1). Für alle in der Datenbank aufgeführten Schrauben mit einem gewindefreien Schaftbereich ist die kleinste Querschnittsfläche dieses gewindefreien Schaftbereichs stets um mehr als 10\% größer als die Querschnittsfläche des Gewindekerns. Die in der Spalte 26 getroffene Abschätzung kann somit als eine Obergrenze für die Zugfestigkeit des Schraubenkernquerschnittes im Gewindebereich angesehen werden. Die tatsächliche Zugfestigkeit kann durch diese Abschätzung aufgrund der Kerbwirkung des Gewindes um bis zu 10\% überschätzt werden (vgl. Abschnitt 2.2.2). In der Tabelle 3.1 werden die Abschätzungen für $R_{m, S G}$ getrennt nach Werkstoffen zusammengefaßt.

Tabelle 3.1: Abschätzungen für die Zugfestigkeiten $R_{m, s G}$ des Gewindekerns

\begin{tabular}{|c|c|c|c|c|c||}
\hline $\begin{array}{c}\text { Werkstoff des } \\
\text { Schraubengewindes }\end{array}$ & Anzahl ausgewer- & \multicolumn{4}{|c||}{$\mathrm{R}_{\mathrm{m}, \mathrm{SG}}=\mathrm{F}_{\mathrm{ZB}} / \mathrm{A}_{\text {Kern }}\left[\mathrm{N} / \mathrm{mm}^{2}\right]$} \\
& teter Schrauben & Mittel & Max. & Min. & Stabw. \\
\hline \hline $\begin{array}{c}\text { nichtrostender, au- } \\
\text { stenitischer Stahl }\end{array}$ & 46 & 930 & 1299 & 730 & 125 \\
\hline $\begin{array}{c}\text { niedriglegierter Koh- } \\
\text { lenstoffstahl }\end{array}$ & 17 & 1190 & 1476 & 919 & 127 \\
\hline
\end{tabular}




\section{$4 \quad$ FE-Untersuchungen}

\subsection{Allgemeines}

Alle Finite-Element- Untersuchungen wurden mit dem Programm ANSYS [36] durchgeführt. Am Anfang einer jeden FE-Berechnung steht die Überlegung, welchen Detaillierungsgrad das Modell aufweisen muß, damit wirklichkeitsnahe Ergebnisse erzielt werden. In [11] wurde bereits versucht, die Auszugversuche über die Verwendung von ebenen Schalenelementen zu simulieren, ohne dabei die räumliche Verzahnung zwischen dem Schraubengewinde und dem Muttergewinde in der Unterkonstruktion darzustellen. Dieser Weg führte jedoch zu keinen befriedigenden Ergebnissen. Deswegen wurde ein FE-Modell entwickelt, in dem sowohl die Gewindegänge der Schraube als auch das in der Unterkonstruktion durch die Schraube geformte Muttergewinde abgebildet wird. Dies wurde erreicht durch Volumenelemente zur Modellierung der Unterkonstruktion und der Gewindegänge der Schrauben sowie durch flächige Kontaktelemente, die dafür sorgen, daß lediglich Druck- und Reibungskräfte zwischen Schrauben- und Muttergewinde übertragen werden.

Mit diesem FE-Modell wird ein Versagen des Schraubengewindes, ein Versagen des Muttergewindes und eine Kombination dieser beiden Versagensarten erfaßt. Das Versagen durch einen Zugbruch der Schraube wird durch das FE-Modell nicht erfaßt, da der Schraubenkopf und auch der Schraubenschaft im Gewindebereich nicht abgebildet werden.

Die Verifizierung des FE-Modells erfolgte durch eine Simulation von Auszugversuchsserien, die aus der im Kapitel 3 beschriebenen Versuchsdatenbank so ausgewählt wurden, daß sowohl für die Schrauben als auch für die Unterkonstruktionen eine möglichst große Bandbreite an unterschiedlichen geometrischen und werkstofflichen Randbedingungen abgedeckt werden.

\subsection{Simulierte Versuchsserien der Datenbank}

Die zur Verifizierung des FE-Modells ausgewählten 25 Auszugversuche der Versuchsdatenbank (Anhang A, Tabellen A.1 und A.2) sind in der Tabelle 4.1 aufgeführt.

Durch die getroffene Auswahl werden Bohrschrauben mit reduzierter Bohrspitze (Br) sowie mit breiter Bohrspitze (Bb), Gewindefurchende Schrauben (GF), Schrauben aus nichtrostendem, austenitischen Stahl (NR) und aus niedriglegiertem Kohlenstoffstahl (C) berücksichtigt. Die Bandbreite der Nennwerte der Gewindeaußendurchmesser $d_{g, N}$ der Schrauben deckt mit $4,8 \mathrm{~mm}$ bis $6,5 \mathrm{~mm}$ den gebräuchlichen Bereich ab. 
Hinsichtlich der Unterkonstruktionen wurden Stahl- (S) und Aluminiumunterkonstruktionen (A) mit Nennblechdicken $t_{N}$ im Bereich von $0,63 \mathrm{~mm}$ bis $5,00 \mathrm{~mm}$ berücksichtigt. Blechdicken der Unterkonstruktion größer $5 \mathrm{~mm}$ wurden nicht untersucht, weil mit Hilfe des entwickelten FE-Modells der Versagensfall des Auszugs der Schraube aus der Unterkonstruktion untersucht werden soll und bei den in der Versuchsdatenbank enthaltenen Auszugversuchen für Stahlunterkonstruktionen mit Blechdicken größer $5 \mathrm{~mm}$ das Versagen fast ausschließlich durch einen Zugbruch der Schrauben erfolgt. Für Aluminiumunterkonstruktionen mit Blechdicken größer $5 \mathrm{~mm}$ liegen in der Datenbank keine Ergebnisse vor.

Tabelle 4.1: Ausgewählte Versuchsserien der Versuchsdatenbank

\begin{tabular}{|c|c|c|c|c|c|c|c|}
\hline & & Vgl. A & $\overline{\text { ang } A}$ & Dellen $A$ & und A.2 & & \\
\hline Versuchs- & & Schr & & & Werkst & der Un & ronstruktion \\
\hline $\begin{array}{c}\text { serie } \\
\text { Lfd.-Nr } \\
\text { der } \\
\text { Datenbank }\end{array}$ & $\begin{array}{l}\text { Lfd.-Nr. } \\
\text { der Da- } \\
\text { tenbank }\end{array}$ & $\begin{array}{c}\text { Werkstoff } \\
\text { NR/C }\end{array}$ & $\begin{array}{l}\mathrm{GF} \\
\mathrm{Br} \\
\mathrm{Bb}\end{array}$ & $\begin{array}{c}\mathrm{d}_{\mathrm{g}, \mathrm{N}} \\
{[\mathrm{mm}]}\end{array}$ & $\begin{array}{c}\text { Stahl/S } \\
\text { Alu/A }\end{array}$ & $\begin{array}{c}t_{N} \\
{[\mathrm{~mm}]}\end{array}$ & $\begin{array}{l}\text { Charge } \\
\text { Lfd.-Nr } \\
\text { der } \\
\text { Datenbank. }\end{array}$ \\
\hline 207 & 67 & NR & $\mathrm{Bb}$ & 5,5 & $\bar{S}$ & 4,00 & 100 \\
\hline 175 & & & & & & 0,63 & 94 \\
\hline 176 & 55 & NR & $\mathrm{Br}$ & 6,3 & $S$ & 1,00 & 96 \\
\hline 177 & & & & & & 1,50 & 98 \\
\hline 191 & & & & & & 1,50 & 98 \\
\hline 192 & 61 & סוN & $\mathrm{Ph}$ & 55 & $c$ & 2,00 & 99 \\
\hline 193 & 01 & INR & DOD & 3,5 & 5 & 3,00 & 93 \\
\hline 194 & & & & & & 4,00 & 100 \\
\hline 172 & & & & & & 0,63 & 94 \\
\hline 173 & 54 & NR & $\mathrm{Br}$ & 4,8 & S & 1,00 & 96 \\
\hline 174 & & & & & & 1,50 & 98 \\
\hline 182 & & & & & & 0,75 & 95 \\
\hline 183 & 57 & סוN & $\mathrm{Ph}$ & 10 & $c$ & 1,00 & 96 \\
\hline 185 & $5 /$ & INR & DD & 4,8 & 5 & 1,50 & 98 \\
\hline 186 & & & & & & 2,00 & 99 \\
\hline 141 & & & & & & 0,63 & 94 \\
\hline 142 & & $\mathrm{NR}$ & CF & 65 & S & 0,75 & 95 \\
\hline 143 & 47 & NR & Git & 6,5 & 5 & 1,00 & 96 \\
\hline 145 & & & & & & 1,50 & 98 \\
\hline 181 & 56 & NR & $\mathrm{Bb}$ & 6,3 & $S$ & 5,00 & 118 \\
\hline 229 & 73 & NR & $R h$ & 5 & & 1,50 & 126 \\
\hline 230 & 13 & $\mathrm{NRR}$ & BD & 5,5 & $\Delta$ & 2,00 & 123 \\
\hline 227 & 72 & NR & $R h$ & 18 & $\mathrm{H}$ & 2,00 & 123 \\
\hline 228 & $1<$ & ח ח & DID & 4,0 & & 3,00 & 124 \\
\hline 205 & 66 & $C$ & $\mathrm{Bb}$ & 6,3 & $S$ & 4,00 & 100 \\
\hline
\end{tabular}


Die für die FE-Modellierung benötigten geometrischen und werkstoffspezifischen Randbedingungen der in der Tabelle 4.1 aufgeführten Versuchsserien sind in den Tabellen 4.2 und 4.3 zusammengestellt.

Aus der Tabelle 4.2 sind folgende von den Schrauben vorgegebenen Randbedingungen zu entnehmen:

- $\quad$ Als Zugfestigkeit ist der in Spalte 26 der Tabelle A.2 der Versuchsdatenbank abgeschätzte Wert übernommen.

- Als Werte für den Gewindeaußendurchmesser $d_{g}$, den Kerndurchmesser $d_{k}$ sowie die Gewindesteigung P sind die in der Tabelle A.2 angegebenen Mittelwerte der Maßprüfungen übernommen.

- Für den Flankenwinkel a wurde für alle Schrauben der von den Herstellern angegebene Nennwert von $60^{\circ}$ angenommen.

- Für die Abflachung $c$ des Gewindeprofils enthält die Versuchsdatenbank keine Angaben, so daß für dieses $M a ß$ eine geeignete Abschätzung getroffen werden mußte. In DIN EN ISO 1478 wird für das Maß c für Blechschrauben mit einem Nennwert des Gewindeaußendurchmessers größer oder gleich 4,8mm ein oberer Grenzwert von 0,15mm angegeben. Dieser Wert wurde für alle Schrauben übernommen. Um die Auswirkung einer Variation der Gewindeabflachung zu untersuchen, wurde zusätzlich für die Schraube Nr. 61 in den FE-Berechnungen ein Wert von 0,10mm für $\mathrm{c}$ angenommen.

- Zur Modellierung des Kernloches, das in der Unterkonstruktion durch die Schraubenmontage erzeugt wird, wurde für den Durchmesser dieses Kernloches $d_{L}$ der größere der beiden Werte aus dem Kerndurchmesser des Schraubengewindes $d_{k}$ und dem Bohrspitzendurchmesser $d_{B s}$ für Bohrschrauben oder dem Vorbohrdurchmesser $d_{B v}$ für gewindefurchende Schrauben angenommen. Für die Bohrspitzendurchmesser wurden die Mittelwerte der Maßprüfungen aus Spalte 21 der Tabelle A.2 angesetzt. Die Vorbohrdurchmesser wurden aus der Spalte 8 der Tabelle A.1 entnommen.

- Die in der letzten Spalte der Tabelle 4.2 angegebene Bezeichnung wird zur Kennzeichnung der unterschiedlichen Schraubentypen verwendet, die in den FE-Berechnungen untersucht wurden. 
Tabelle 4.2: Durch die Schrauben vorgegebene Randbedingungen für die FE-

Die Eigenschaften der Unterkonstruktionen, die durch das FE-Modell abgebildet werden müssen, sind in der Tabelle 4.3 angegeben.

- Die Werte für die 0,2\%-Dehngrenzen $R_{p 0,2}$ und die Zugfestigkeiten $R_{m}$ wurden aus der Tabelle A.1 entnommen.

- Die Blechdicken $t_{k}$ für eine Werkstoffcharge wurden festgelegt, indem für alle Versuchsserien, die in der Tabelle 4.1 für die jeweilige Werkstoffcharge aufgeführt sind, der Mittelwert der an den für die Unterkonstruktion verwendeten Probekörper gemessenen Kernblechdicken aus der Tabelle A.1 gebildet wurde.

- In Übereinstimmung mit den Angaben der Tabelle A.1 und dem im Abschnitt 2.3 im Bild 2.14 dargestellten Versuchsaufbau für die Auszugversuche wurde die Unterkonstruktion im FE-Modell als U-Profil $70 \mathrm{~mm} \times 30 \mathrm{~mm}$ mit einer Stützweite $I_{S}=80 \mathrm{~mm}$ abgebildet.

- Die in der letzten Spalte der Tabelle 4.3 angegebene Bezeichnung wird zur Kennzeichnung der jeweiligen Unterkonstruktion verwendet, die in den FE-Berechnungen untersucht wurde. Diese Bezeichnung setzt sich zusammen aus der mit 100 multiplizierten Nennblechdicke der Unterkonstruktion und dem Zusatz „S“ für eine Stahlunterkonstruktion oder dem Zusatz „A“ für eine Aluminiumunterkonstruktion. 
Tabelle 4.3: Randbedingungen für die Unterkonstruktionen

\begin{tabular}{|c|c|c|c|c|c|}
\hline Chargen Nr. & $\begin{array}{c}\mathrm{R}_{\mathrm{p} 0,2} \\
{\left[\mathrm{~N} / \mathrm{mm}^{2}\right]}\end{array}$ & $\begin{array}{c}\mathrm{R}_{\mathrm{m}} \\
{\left[\mathrm{N} / \mathrm{mm}^{2}\right]}\end{array}$ & $\mathrm{t}_{\mathrm{K}}[\mathrm{mm}]$ & Probenform & FE-Bez. \\
\hline 94 & 346 & 415 & 0,60 & \multirow{11}{*}{$\begin{array}{l}\text { U-70x30, } \\
\text { Stützweite: } \\
\mathrm{I}_{\mathrm{S}}=80 \mathrm{~mm}\end{array}$} & $63 S$ \\
\hline 95 & 266 & 387 & 0,69 & & $75 S$ \\
\hline 96 & 396 & 416 & 0,98 & & $100 S$ \\
\hline 98 & 261 & 369 & 1,47 & & $150 S$ \\
\hline 99 & 213 & 348 & 1,99 & & $200 S$ \\
\hline 93 & 177 & 301 & 3,06 & & $300 S$ \\
\hline 100 & 324 & 419 & 3,94 & & $400 S$ \\
\hline 118 & 235 & 351 & 4,90 & & $500 S$ \\
\hline 126 & 162 & 232 & 1,47 & & $150 \mathrm{~A}$ \\
\hline 123 & 164 & 238 & 2,02 & & $200 A$ \\
\hline 124 & 176 & 233 & 2,94 & & $300 A$ \\
\hline
\end{tabular}

\subsection{Verwendete Elemente}

Es werden die Elemente Solid45, CONTA173, TARGE170 und CONT52 entsprechend der folgenden Erläuterung zur Modellierung verwendet.

\section{„Solid45“:}

Diese Volumenelemente werden zur Abbildung der Unterkonstruktion sowie des Schraubengewindes verwendet. Es werden dabei sowohl quaderförmige Elemente mit acht Knoten als auch tetraederförmige Elemente mit vier Knoten verwendet.

Die Elemente haben pro Knoten als Freiheitsgrade die drei translatorischen Verschiebungen in Richtung eines kartesischen Koordinatensystems.

Zwischen den Knoten eines Elements wird der Verlauf der Geometrie sowie der Verlauf der Verschiebungen durch lineare Ansatzfunktionen approximiert.

Die Elemente wurden vollständig integriert (Einstellung in Ansys: KEYOPT(2)=0), und zur Vermeidung von Schubversteifungseffekten, die aufgrund der vollständigen Integration auftreten können, wurden Ansatzfunktionen für die Approximation der Schubverzerrungen über die Einstellung KEYOPT(1)=0 aktiviert.

\section{„CONTA173“ und „,TARGE170“:}

Diese zweidimensionalen Elemente dienen zur Beschreibung eines flächigen Kontaktes. Sie werden immer paarweise verwendet, um diejenigen Flächen zweier nicht zusammenhängender Körper zu definieren, die in Kontakt treten können. Für die Kontaktmodellierung zwi- 
schen zwei nachgiebigen Körpern - dieser Fall liegt für das im Rahmen dieser Arbeit entwikkelte FE-Modell vor - dienen die Kontaktelemente nicht zur Modellierung der Kontaktkörper, sondern werden lediglich auf die Oberflächen von bereits vorhanden Elementen (z. B. Solid45) gelegt, um die möglichen Kontaktzonen zu definieren. Die Verformungen und Spannungen beim Auftreten eines Kontaktes werden durch die unterhalb der Kontaktelemente liegenden Elemente bestimmt.

Die Kontaktelemente können auch beim Auftreten eines Kontaktes mit vorgegebenen Reibungskoeffizienten Reibungskräfte rechtwinklig zur Kontaktkraft abtragen.

\section{„CONTA52“:}

Dies sind eindimensionale Kontaktelemente, die so eingestellt werden können, daß sie nur eine Druckkraft in Richtung ihrer Achse übertragen können. Sie werden für die Lagerung der Bügelprobe verwendet.

\subsection{Geometrie und Vernetzung}

\subsubsection{Schrauben}

Die Schrauben wurden in dem FE-Modell mit ihren Gewindegängen abgebildet. Die Gewindegänge wurden mit Hilfe von quaderförmigen, 8-knotigen Volumenelemente Solid45 modelliert.

Zur Erstellung der Geometrie und des Elementnetzes (Bilder 4.1 und 4.2) wurden für die verschiedenen Schraubentypen die Angaben der Tabelle 4.2 für den

- Gewindeaußendurchmesser $d_{g}$,

- den Kerndurchmesser $d_{K}$,

- die Gewindesteigung P,

- die Gewindeabflachung c,

- den Flankenwinkel $\alpha$

- $\quad$ und den Kernlochdurchmesser im Muttergewinde $d_{L}$

verwendet. Die Höhe der Basis b ergibt sich über die festgelegten Werte für $d_{g}, d_{\kappa}, c$ und $\alpha$.

Die Vernetzung erfolgte durch Vorgabe der Anzahl der Elemente über die Höhe der Gewindeflanken und der Anzahl der Elemente in zirkularer sowie in radialer Richtung (Bilder 4.1 und 4.2). Für alle untersuchten Schraubentypen wurden vier Elemente über die Höhe der Gewindeflanken und 24 Elemente über den Umfang $\left(360^{\circ}\right)$ verwendet. Die radiale Unterteilung der Schraubenflanken wird aus dem Bild 4.2 und der Tabelle 4.4 ersichtlich. Die Unter- 
teilung erfolgte für die unterschiedlichen Schraubentypen unter Berücksichtigung der folgenden Gesichtspunkte:

- Für Schrauben, bei denen sich der Bereich der Verzahnung mit der Unterkonstruktion von ihrem Gewindeaußendurchmesser $d_{g}$ bis hin zu ihrem Kerndurchmesser $d_{k}$ erstreckt (Bild 4.2a) - dieser Fall liegt für Bohrschrauben mit einer reduzierten Bohrspitze (Schrauben „B“ und „D“ in Tabelle 4.4) sowie für die gewindefurchende Schraube „F“ für Unterkonstruktionsdicken $t_{N}<1,25 \mathrm{~mm}$ (Schraube „F1“ in der Tabelle 4.4) vor - wurden die Gewindeflanken in radialer Richtung äquidistant unterteilt (Bild 4.2a)). Die Anzahl der Elemente in radialer Richtung nr1 wurde für diese Schrauben so gewählt, daß etwa gleich große radiale Elementlängen Ir1 für die unterschiedlichen Schrauben erzielt wurden (vgl. Tabelle 4.4).

- Für alle anderen Schrauben (Schrauben „A“, „C“, „Cvc“, „E“, „F2“, „G“, „H“, „I“ und „J“ in Tabelle 4.4) erfolgte die radiale Unterteilung der Gewindeflanken getrennt für den Bereich der Verzahnung mit dem Muttergewinde und den Bereich, der nicht vom Muttergewinde überdeckt wird (Bild 4.2b)). Die radiale Unterteilung dieser Bereiche ergibt sich dabei einerseits aus der Bedingung, daß mindestens ein Element in radialer Richtung in jedem Bereich angeordnet wird und andererseits aus dem Bestreben, radiale Elementlängen Ir1 und Ir2 in beiden Bereichen zu erzielen, die etwa in der Größenordung der radialen Elementlängen der erstgenannten Schrauben (Schrauben „B“, „D“ und „F1“) liegen (vgl. Tabelle 4.4).

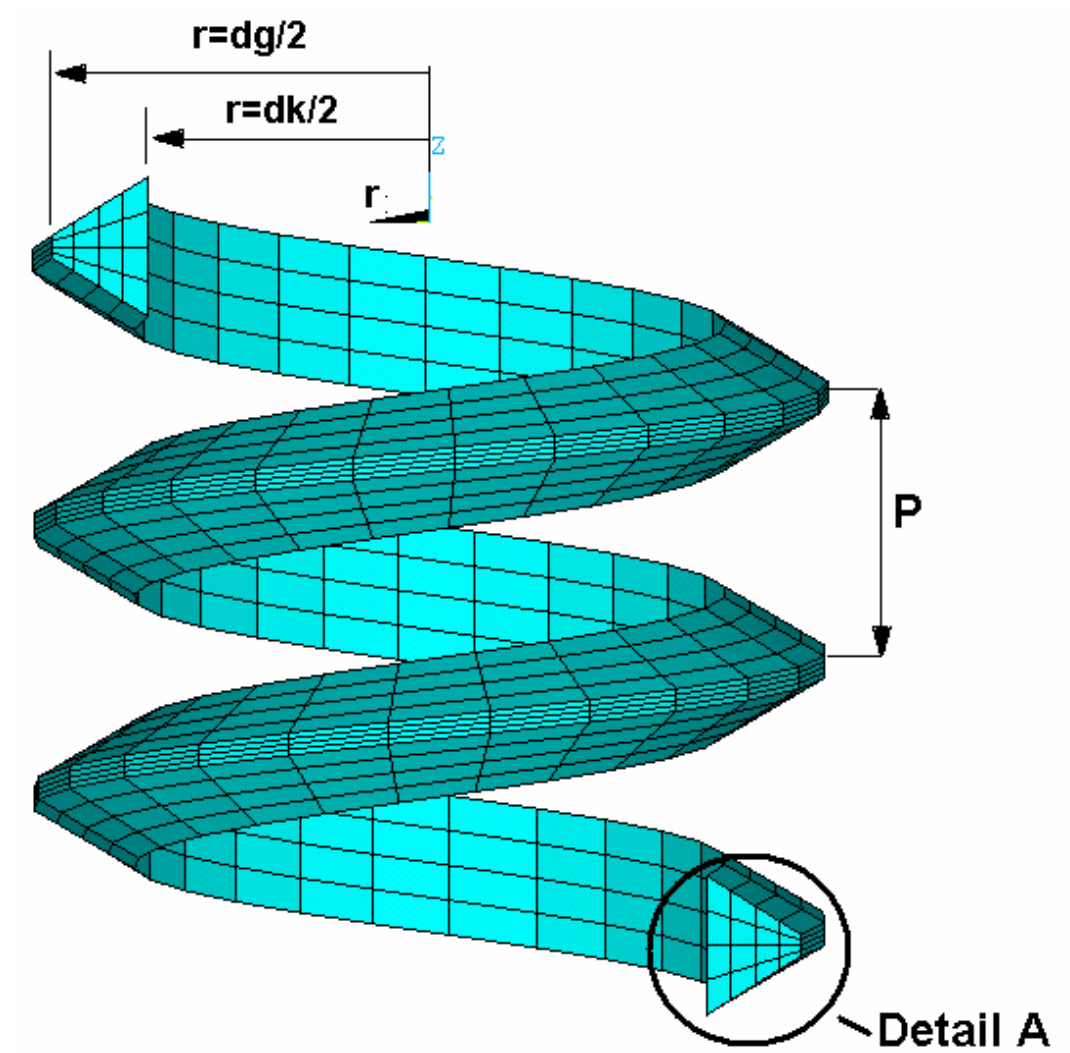

Bild 4.1: Modellierung der Gewindegänge der Schrauben 


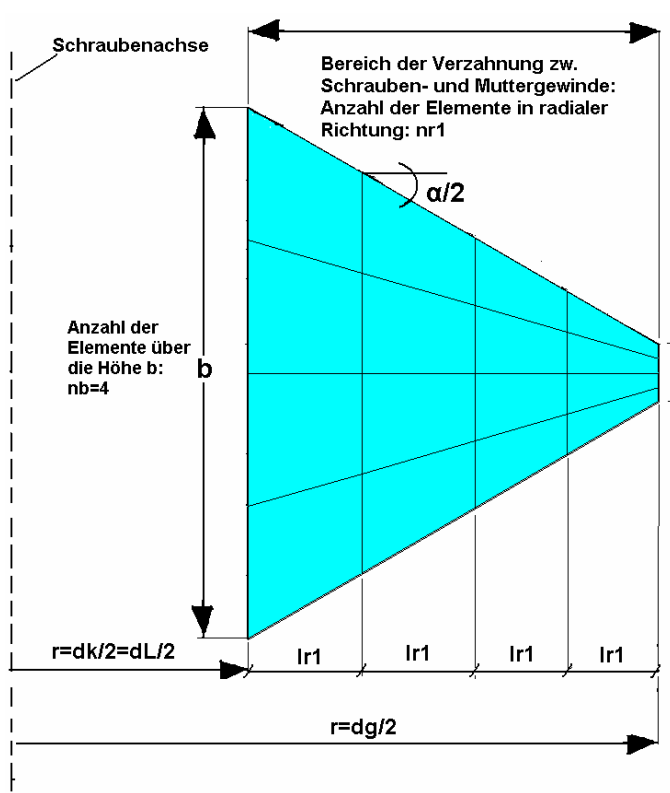

a) Für den Fall $d_{L}=d_{K}$

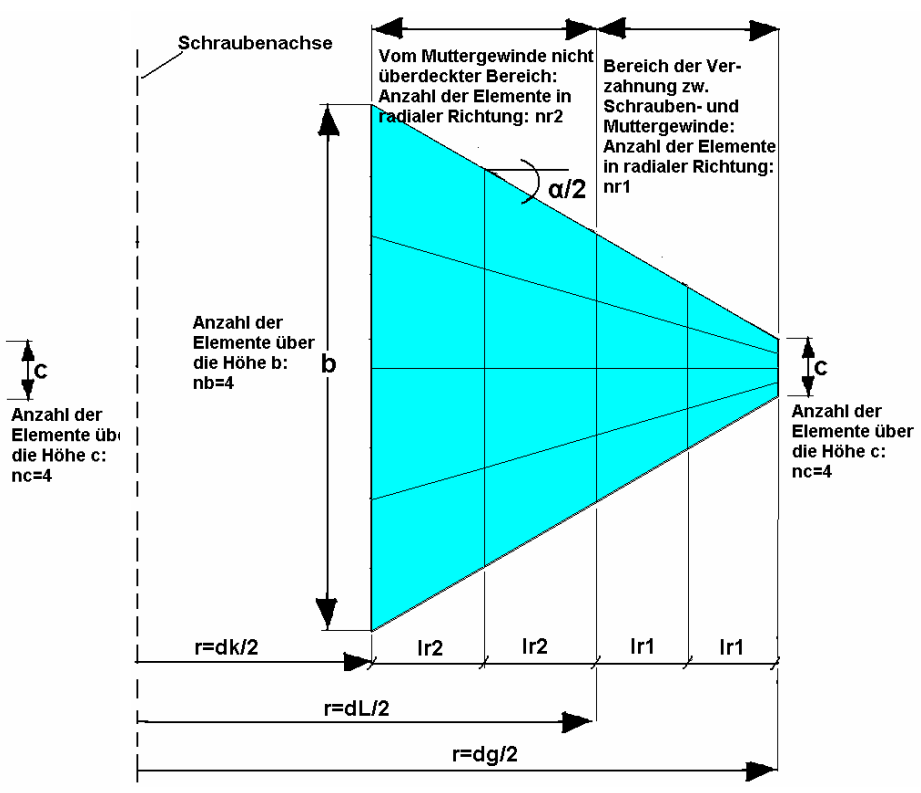

b) Für den Fall $d_{L}>d_{K}$

Bild 4.2: Vernetzter Querschnitt der Gewindegänge, Detail A aus Bild 4.1

Tabelle 4.4: Radiale Vernetzung des Schraubengewindes

\begin{tabular}{|c|c|c|c|c|c|}
\hline $\begin{array}{l}\text { Schraube } \\
\text { Lfd.-Nr. der }\end{array}$ & FE-Bez. & $\begin{array}{r}\text { Bereich der V } \\
\text { Schrauben- u. } \\
\text { vgl. Bilder } 4\end{array}$ & $\begin{array}{l}\text { zahnung zw. } \\
\text { uttergewinde } \\
\text { ) und } 4.2 \mathrm{~b} \text { ) }\end{array}$ & $\begin{array}{r}\text { Bereich der nic } \\
\text { winde üb } \\
\text { vgl. B }\end{array}$ & $\begin{array}{l}\text { om Mutterge- } \\
\text { eckt wird } \\
\text { 4.2b) }\end{array}$ \\
\hline Datenbank & & $\begin{array}{c}\text { radiale Anzahl } \\
\text { der Elemente } \\
\text { nr1 }\end{array}$ & $\begin{array}{l}\text { radiale Ele- } \\
\text { mentlänge } \\
\text { Ir1 [mm] }\end{array}$ & $\begin{array}{l}\text { radiale Anzahl } \\
\text { der Elemente } \\
\text { nr2 }\end{array}$ & $\begin{array}{l}\text { radiale Ele- } \\
\text { mentlänge } \\
\text { Ir2 [mm] }\end{array}$ \\
\hline$\overline{67}$ & $\overline{A A}$ & $\overline{c 1}$ & 0,15 & $\overline{3}$ & 0,22 \\
\hline 55 & $B$ & 4 & 0,17 & - & - \\
\hline 61 & C u. Cvc & 2 & 0,16 & 2 & 0,20 \\
\hline 54 & $\mathrm{D}$ & 4 & 0,20 & - & - \\
\hline 57 & $E$ & 3 & 0,17 & 1 & 0,11 \\
\hline $\begin{array}{c}47 \text { und } \\
t_{N}<1,25 \mathrm{~mm}\end{array}$ & $\mathrm{~F} 1$ & 5 & 0,18 & - & - \\
\hline $\begin{array}{c}47 \text { und } \\
t_{N}=1,5 \mathrm{~mm}\end{array}$ & $\mathrm{~F} 2$ & 4 & 0,17 & 1 & 0,23 \\
\hline 56 & $G$ & 2 & 0,19 & 2 & 0,14 \\
\hline 73 & $\mathrm{H}$ & 3 & 0,15 & 1 & 0,20 \\
\hline 72 & I & 2 & 0,18 & 2 & 0,15 \\
\hline 66 & $\mathrm{~J}$ & 2 & 0,15 & 2 & 0,22 \\
\hline
\end{tabular}




\subsubsection{Unterkonstruktion}

Zur Modellierung der Unterkonstruktion wurde ihre Geometrie zunächst ohne die Berücksichtung der Form des Muttergewindes in vier Bereiche unterteilt (Bild 4.3).
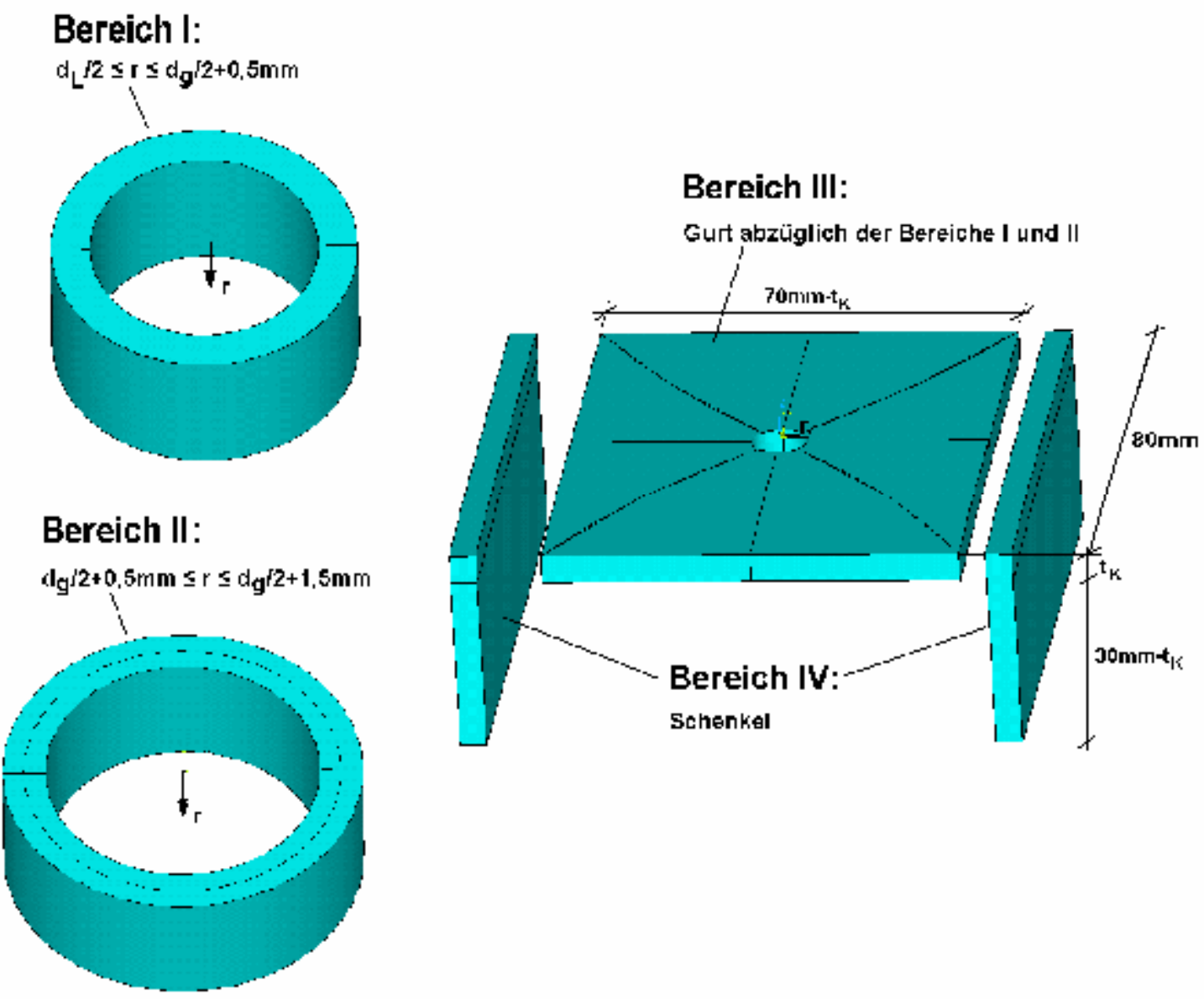

Bild 4.3:Geometrische Unterteilung der Unterkonstruktion

Der Bereich I wird gebildet durch einen Zylinder, dessen Innendurchmesser dem Kernlochdurchmesser $d_{L}$ des Muttergewindes entspricht und dessen Außendurchmesser um $1 \mathrm{~mm}$ größer ist als der Gewindeaußendurchmesser $d_{g}$ der jeweils untersuchten Schraube. In diesem Bereich wird die Form des Muttergewindes erzeugt, indem aus dem Zylinder dasjenige Volumen ausgeschnitten wird, das die geometrische Form der jeweils untersuchten Schraubenflanken besitzt. Die Subtraktion der beiden Volumen wurde mit Hilfe der in Ansys verfügbaren booleschen Verknüpfungsfunktion „Vsbv“ realisiert. Die Vernetzung des neu entstandenen Volumens (Bild 4.4) erfolgte über die Verwendung von 4-knotigen, tetraederförmigen Volumenelementen Solid45. Als Vernetzungsparameter wurden die Anzahl der Elemente über die Blechdicke im Schnitt $r=d g / 2+0,5 \mathrm{~mm}$ und die Anzahl der Elemente in zirkularer sowie in radialer Richtung vorgegeben. In allen durchgeführten Berechnungen wurde eine Anzahl von sechs Elementen über die Blechdicke im Schnitt $r=d g / 2+0,5 \mathrm{~mm}$ verwendet und analog zu der Vernetzung der Schraubenflanken wurden 24 Elemente über den Umfang verwendet (Bild 4.4). Die radiale Unterteilung der Elemente wurde so gewählt, daß die Ver- 
netzung des Muttergewindes mit der Vernetzung der Gewindeflanken der Schrauben im Bereich der Verzahnung zwischen Mutter- und Schraubengewinde übereinstimmte. Dies bedeutet, daß im Verzahnungsbereich zwischen Mutter- und Schraubengewinde die radiale Unterteilung des Muttergewindes in Abhängigkeit von der jeweils untersuchten Schraube gemäß den Angaben für nr1 und Ir1 in Tabelle 4.4 erfolgte.

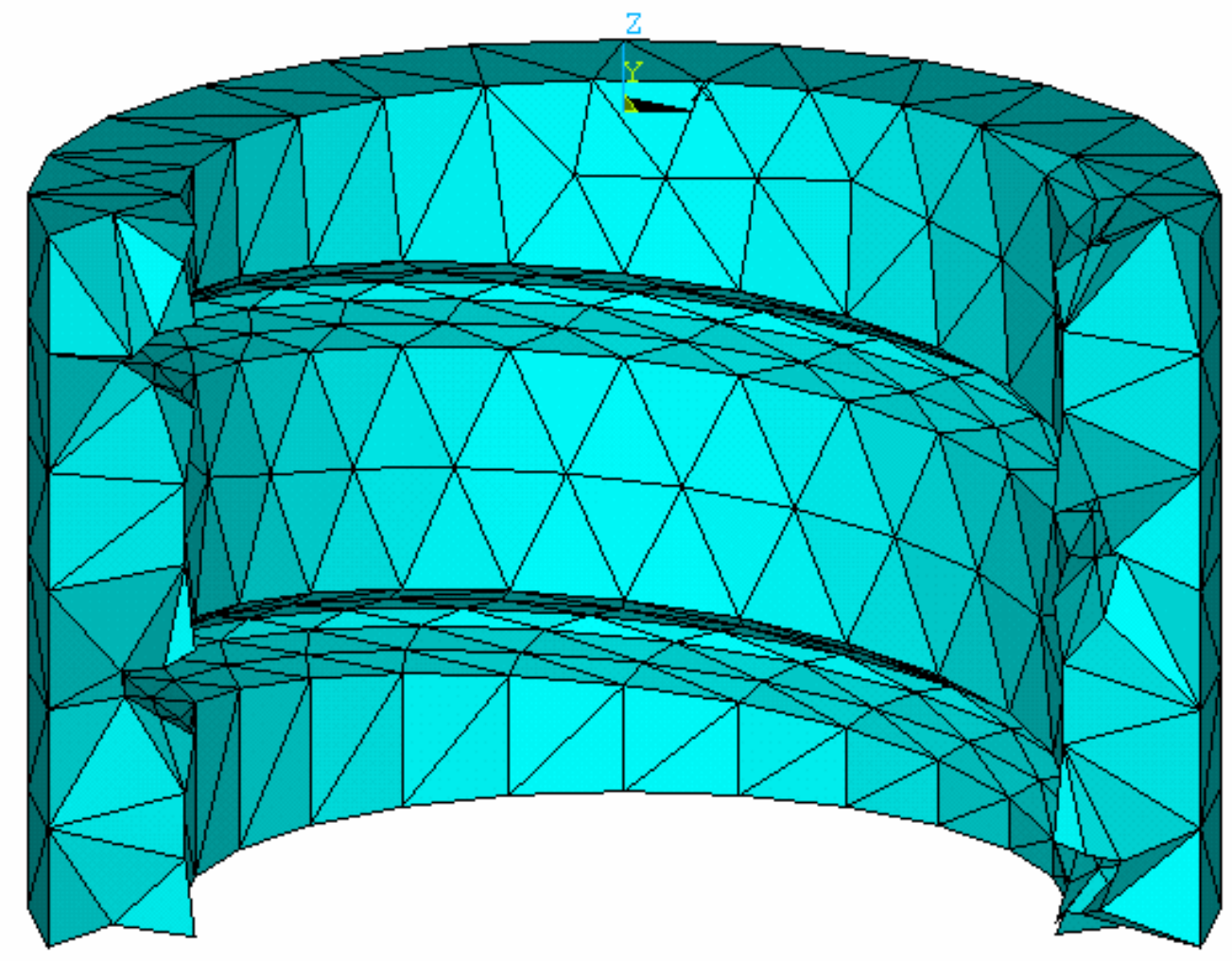

Bild 4.4: Schnitt durch den vernetzten Bereich, in dem das Muttergewinde abgebildet wird

Die Vernetzung für den in Bild 4.3 dargestellten Bereich III der Unterkonstruktion ist im Bild 4.5 dargestellt. Die Vernetzung erfolgte mit Hilfe von quaderförmigen, 8-knotigen Volumenelementen Solid45. Über die Blechdicke der Unterkonstruktion wurden zwei Elemente angeordnet. Die radiale Vernetzung ergibt sich aus der Vorgabe einer durchschnittlichen radialen Elementgröße von $2 \mathrm{~mm}$, wobei die Elemente nach außen hin kontinuierlich vergröBert wurden - das Verhältnis gebildet aus der radialen Elementlänge der äußeren Elemente zu der radialen Elementlänge der inneren Elemente ist ca. drei. In zirkularer Richtung wurde die gleiche Anzahl von Elementen verwendet, wie für den Bereich, in dem das Muttergewinde abgebildet wird (Bilder 4.4 und 4.5). 


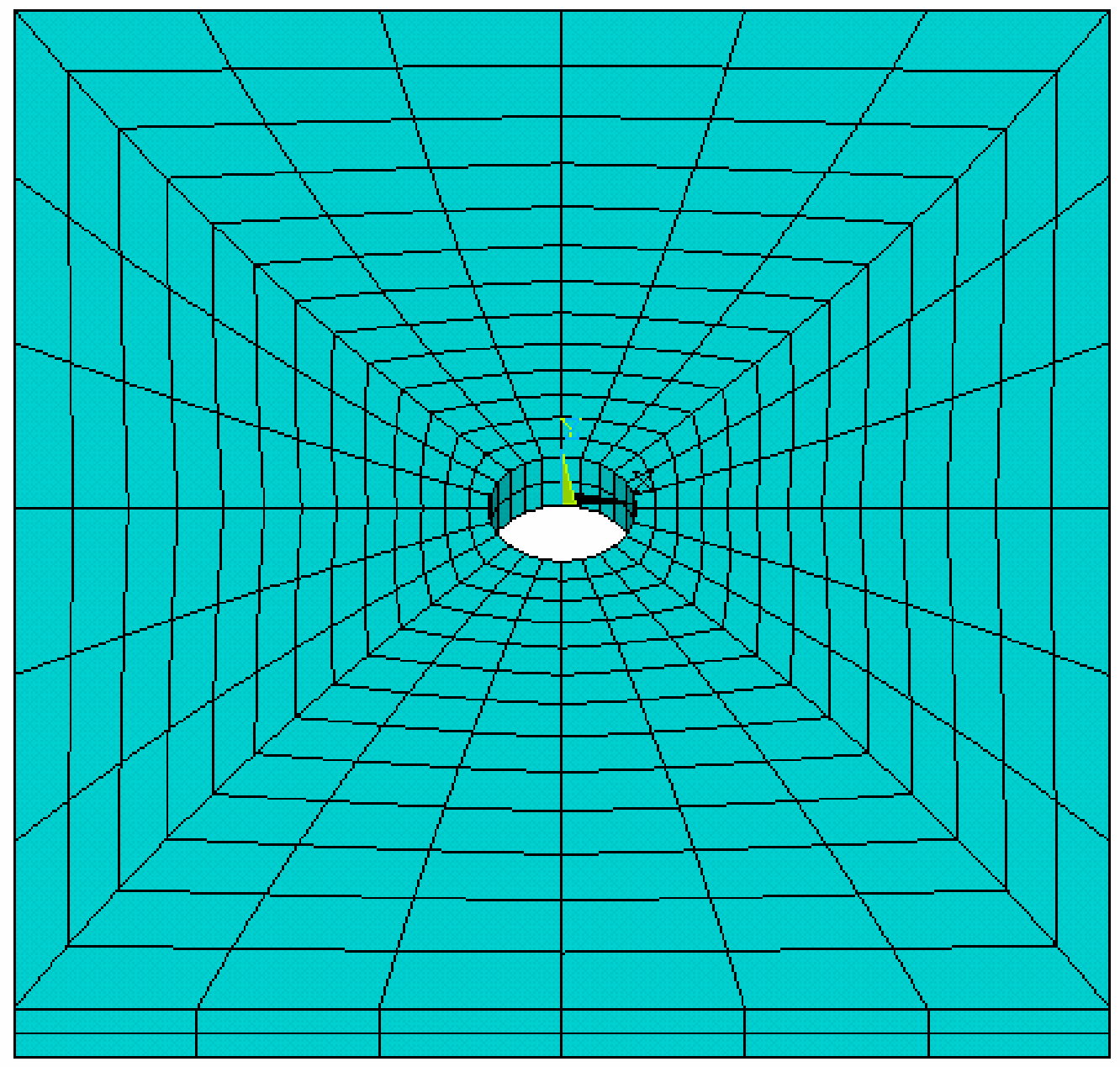

Bild 4.5: Vernetzung des Bereichs III

Mit Hilfe des in Bild 4.3 dargestellten Bereichs II wurde das Elementnetz von den Vernetzungsrandbedingungen des Bereichs, in dem das Muttergewinde abgebildet wird (Bild 4.4) auf die für den Bereich III gewählten Vernetzungsrandbedingungen (Bild 4.5) angepaßt. Da für den Bereich III die gleiche Anzahl von Elementen in zirkularer Richtung verwendet wurde wie für den Bereich, in dem das Muttergewinde abgebildet wird, mußte der Übergangsbereich letztlich nur die unterschiedliche Anzahl der Elemente über die Blechdicke der Unterkonstruktion (Übergang von sechs auf zwei Elemente) ausgleichen. Der Bereich II konnte somit mit Hilfe von quaderförmigen, 8-knotigen Volumenelemente Solid45 abgebildet werden (Bild 4.6). 


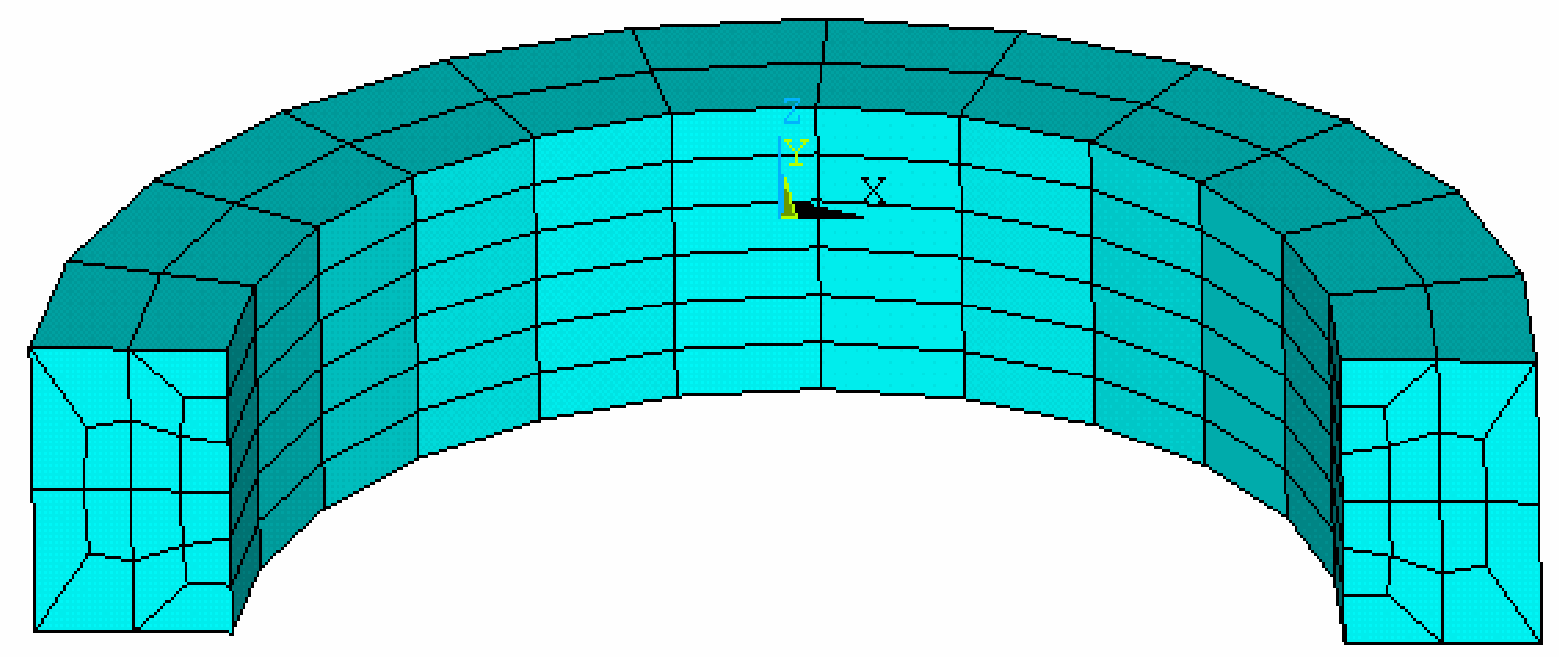

Bild 4.6: Schnitt durch den vernetzten Bereich II

Im Bereich IV werden die Schenkel der Unterkonstruktion abgebildet (Bild 4.7). Die Vernetzung an den Längsrändern ergibt sich aus den Vorgaben des Bereichs III - also eine Anzahl von sechs Elementen über die Spannweite der Unterkonstruktion und eine Anzahl von zwei Elementen über die Gurtdicke. Über die verbleibende Schenkelhöhe wurde eine Elementlänge von $2 \mathrm{~mm}$ vorgegeben und über die Schenkeldicke wurden zwei Elemente angeordnet.
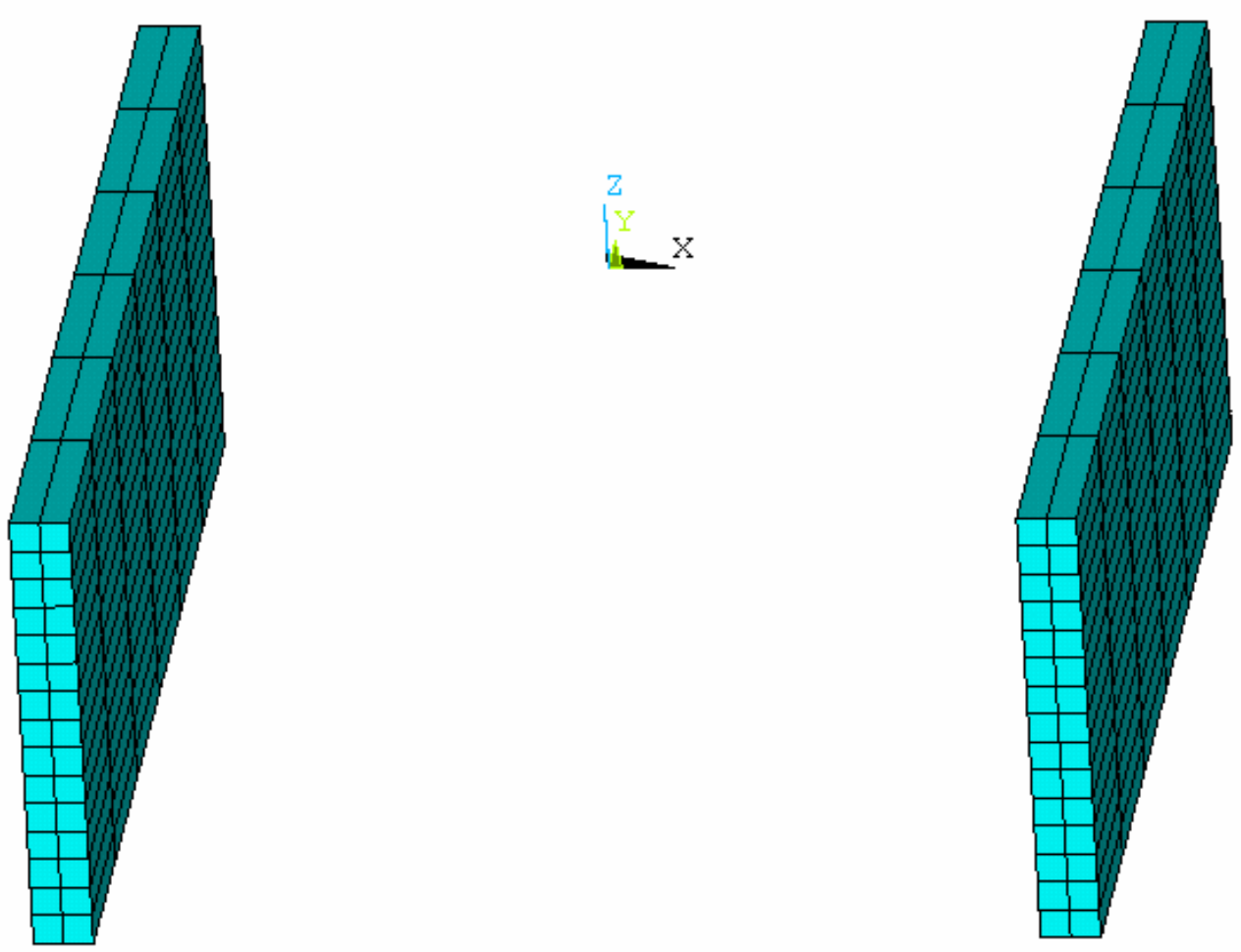

Bild 4.7: Vernetzung des Bereichs IV 


\subsection{Berechnungsansätze zur Beschreibung des Werkstoffverhaltens der Unterkonstruktionen und der Schrauben}

Um die Verformungsverhältnisse der Auszugversuche im Verzahnungsbereich zwischen Muttergewinde und Schraubengewinde so wirklichkeitsnah wie möglich abzubilden - in diesem Bereich ist aufgrund der Lasteinleitung mit großen plastischen Verformungen zu rechnen - wurden zur Beschreibung des Werkstoffverhaltens der Unterkonstruktionen sowie der Gewindegänge der Schrauben nichtlineare Werkstoffgesetze verwendet und die FEBerechnungen wurden geometrisch nichtlinear durchgeführt.

Die in den FE-Berechnungen verwendeten Werkstoffgesetze sind im Anhang B aufgeführt (in den Diagrammen B.1 bis B.14 die Werkstoffgesetze für die Unterkonstruktionen und in den Diagrammen B.15 bis B.19 die Werkstoffgesetze für die Schrauben).

Zur Beschreibung des Werkstoffverhaltens der in den FE-Berechnungen untersuchten Unterkonstruktionen wurde zunächst für jede Unterkonstruktion ein Werkstoffgesetz aus den Spannungs-Dehnungsverläufen der einaxialen Zugversuche für die jeweilige Unterkonstruktion abgeleitet, das sowohl auf die Bereiche außerhalb des Muttergewindes angewendet wurde als auch auf den Bereich, in dem das Muttergewinde erzeugt wird.

Das Vorgehen zur Ermittlung der Werkstoffgesetze für die in der Tabelle 4.3 aufgeführten Unterkonstruktionen ist beispielhaft im Diagramm 4.1 für die Charge Nr. 94=63S dargestellt.

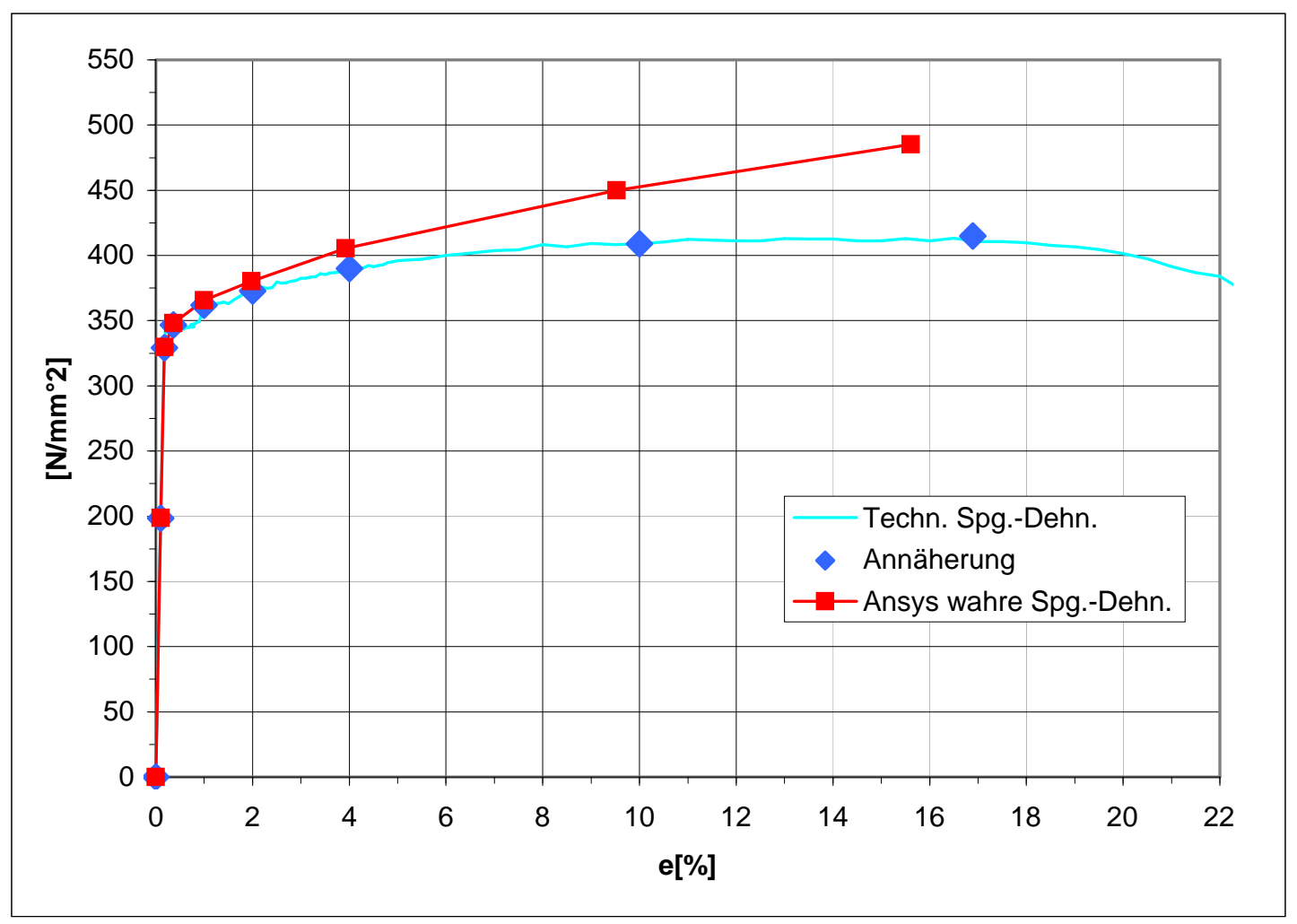

Diagramm 4.1: Werkstoffgesetz für die Charge Nr. 94=63S 
Für alle Unterkonstruktionen der Tabelle 4.3 lagen die Spannungs-Dehnungsverläufe der einaxialen Zugversuche - hierbei handelt es sich um technische Spannungen und Dehnungen - vor. Aus den für eine Unterkonstruktion vorliegenden Zugversuchen wurde eine mittlere Spannungs-Dehnungsbeziehung gebildet, indem die Spannungswerte an Stellen gleicher Dehnungen gemittelt wurden (Kurve „Techn. Spg.-Dehn.“ im Diagramm 4.1). Diese mittlere Spannungs-Dehnungsbeziehung wurde dann bis zu dem Punkt, an dem die technische Zugfestigkeit erreicht war, durch maximal neun Spannungs-Dehnungspunkte angenähert („Annäherung“ im Diagramm 4.1). Aufgrund der geometrisch nichtlinearen Berechnung wird zur korrekten Beschreibung des Werkstoffgesetzes in ANSYS die wahre SpannungsDehnungsbeziehung benötigt. Hierzu wurden die technischen Spannungen und Dehnungen $\left(\sigma_{\text {tech }}, \varepsilon_{\text {tech }}\right)$ über die Beziehungen (4.1) und (4.2) in wahre Spannungen und Dehnungen $\left(\sigma_{\text {wahr }}, \varepsilon_{\text {wahr }}\right)$ umgerechnet (Kurve „Ansys wahre Spg.-Dehn.“ im Diagramm 4.1).

$\sigma_{\text {wahr }}=\sigma_{\text {tech }}\left(1+\varepsilon_{\text {tech }}\right)$

$\varepsilon_{\text {wahr }}=\ln \left(1+\varepsilon_{\text {tech }}\right)$

Diese wahren Spannungs-Dehnungspunkte wurden in ANSYS unter Verwendung des multilinearen Werkstoffgesetzes „MISO“ eingegeben. Über den letzten eingegebenen Spannungs-Dehnungspunkt hinaus unterstellt ANSYS einen horizontalen Verlauf für die Spannungs-Dehnungsbeziehung. Die auf diese Weise ermittelten Werkstoffgesetze für die in der Tabelle 4.3 aufgeführten Unterkonstruktionen sind im Anhang B in den Diagrammen B.1 bis B.11 dargestellt.

Die in der Versuchsdatenbank in der Tabelle A.1 aufgeführten Stahlunterkonstruktionen weisen fast ausschließlich Zugfestigkeiten auf, die innerhalb des in DIN EN 10025-2 für die Festigkeitsklasse S235 und in DIN EN 10326 für die Festigkeitsklasse S280 angegebenen Bereichs liegen. Für die Zukunft der Leichtbauweise ist für die gewindeformenden Schrauben eine Ausweitung ihrer Anwendungsgrenzen auf Unterkonstruktionen der Festigkeitsklassen S355 gemäß DIN EN 10025-2 und S350 gemäß DIN EN 10326 zu erwarten. Zur Berücksichtigung dieser Entwicklungen wurde in den Parameterstudien für die Unterkonstruktion das im Anhang B im Diagramm B.12 dargestellte Werkstoffgesetz untersucht. Die zur Beschreibung dieses Werkstoffverhaltens zugrundegelegte technische Spannungs-Dehnungsbeziehung resultiert aus Zugversuchen, die im Rahmen von Werkstoffprüfungen an der Versuchsanstalt für Stahl, Holz und Steine an einem Stahl durchgeführt wurden, der eine Zugfestigkeit von $\mathrm{R}_{\mathrm{m}}=560 \mathrm{~N} / \mathrm{mm}^{2}$ besitzt, die der nach DIN EN 10326 maximal zu erwartenden Zugfestigkeit der Festigkeitsklasse S350 entspricht, und die etwa im mittleren Bereich der in DIN EN 10025-2 für die Festigkeitsklasse S355 angegebenen Zugfestigkeiten liegt. Die in der Tabelle A.1 für die Versuchsserie Nr. 28 aufgeführte Stahlunterkonstruktion Nr. 15 weist mit $741 \mathrm{~N} / \mathrm{mm}^{2}$ als einzige Stahlunterkonstruktion der Versuchsdatenbank eine Zugfestigkeit 
auf, die deutlich größer ist als $560 \mathrm{~N} / \mathrm{mm}^{2}$. Der Einsatzbereich der gewindeformenden Schrauben für Unterkonstruktionen mit Zugfestigkeiten, die in dem Bereich der Zugfestigkeit der Stahlunterkonstruktion Nr. 15 liegen, ist aufgrund der Gewährleistung eines einwandfreien Gewindeformvorgangs lediglich auf sehr kleine Blechdicken beschränkt (die Unterkonstruktion Nr. 15 besitzt eine Kernblechdicke von 0,58mm). Dieser Einsatzbereich wird auch durch zukünftige Entwicklungen im Leichtbau nicht auf größere Blechdicken ausgeweitet werden können. Aus diesem Grund wurden Werkstoffestigkeiten, die im Bereich der Zugfestigkeit der Stahlunterkonstruktion Nr. 15 liegen, in den Parameterstudien nicht untersucht.

Zur Beurteilung und Abschätzung des Effektes einer Kaltverfestigung, die sich im Muttergewinde während des Gewindeformvorgangs eventuell einstellt, wurden Kleinlasthärteprüfungen an einem Schnitt durch das Muttergewinde durchgeführt, das in der Unterkonstruktion Nr. 100=400S der Tabelle 4.3 durch den Gewindeformvorgang mit den Schrauben Nr. 67=A und Nr. 61=C=Cvc der Tabelle 4.2 erzeugt wurde. Die in den Diagrammen 4.2 und 4.3 dargestellten Ergebnisse dieser Härteprüfungen verdeutlichen folgendes:

- Der Effekt der Kaltverfestigung ist an den Oberflächen der Gewindegänge des Muttergewindes deutlich zu erkennen.

- Diese Kaltverfestigung klingt mit zunehmenden Abstand von den Oberflächen des Gewindes sowohl in radialer Richtung $x$ als auch in axialer Richtung (Übergang von den Härteverläufen 1_oben und 1_unten zu dem Härteverlauf 1_mitte) sehr rasch ab.

- Außerhalb des Bereiches, in dem das Muttergewinde im FE-Modell abgebildet wird (Bereich I im Bild 4.3, Abschnitt 4.4.2), ist der Effekt der Kaltverfestigung bereits nahezu vollständig abgeklungen. 


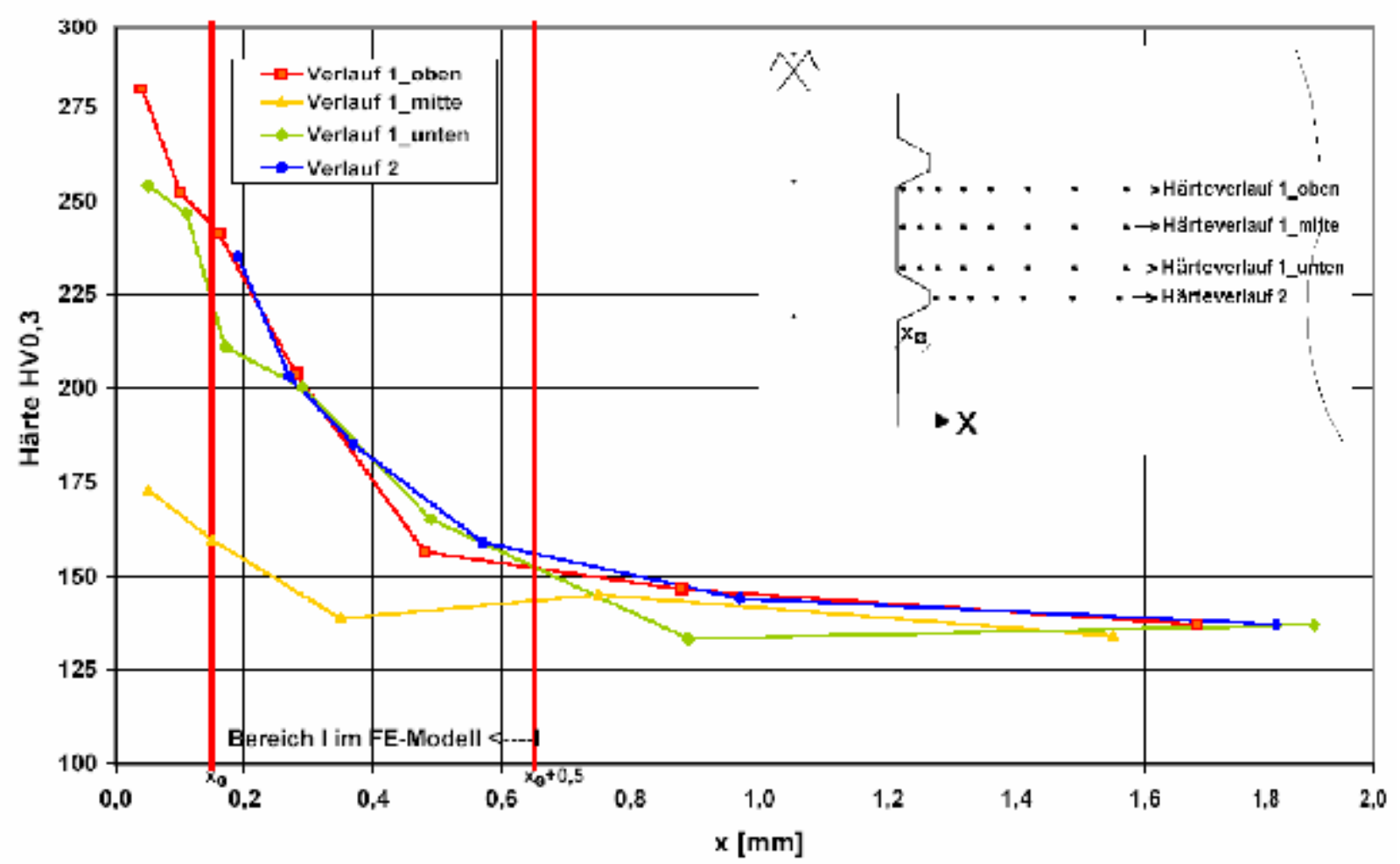

Diagramm 4.2: Härteprüfungen in der Unterkonstruktion Nr. 100=400S nach dem Gewindeformvorgang mit der Schraube Nr. 67=A

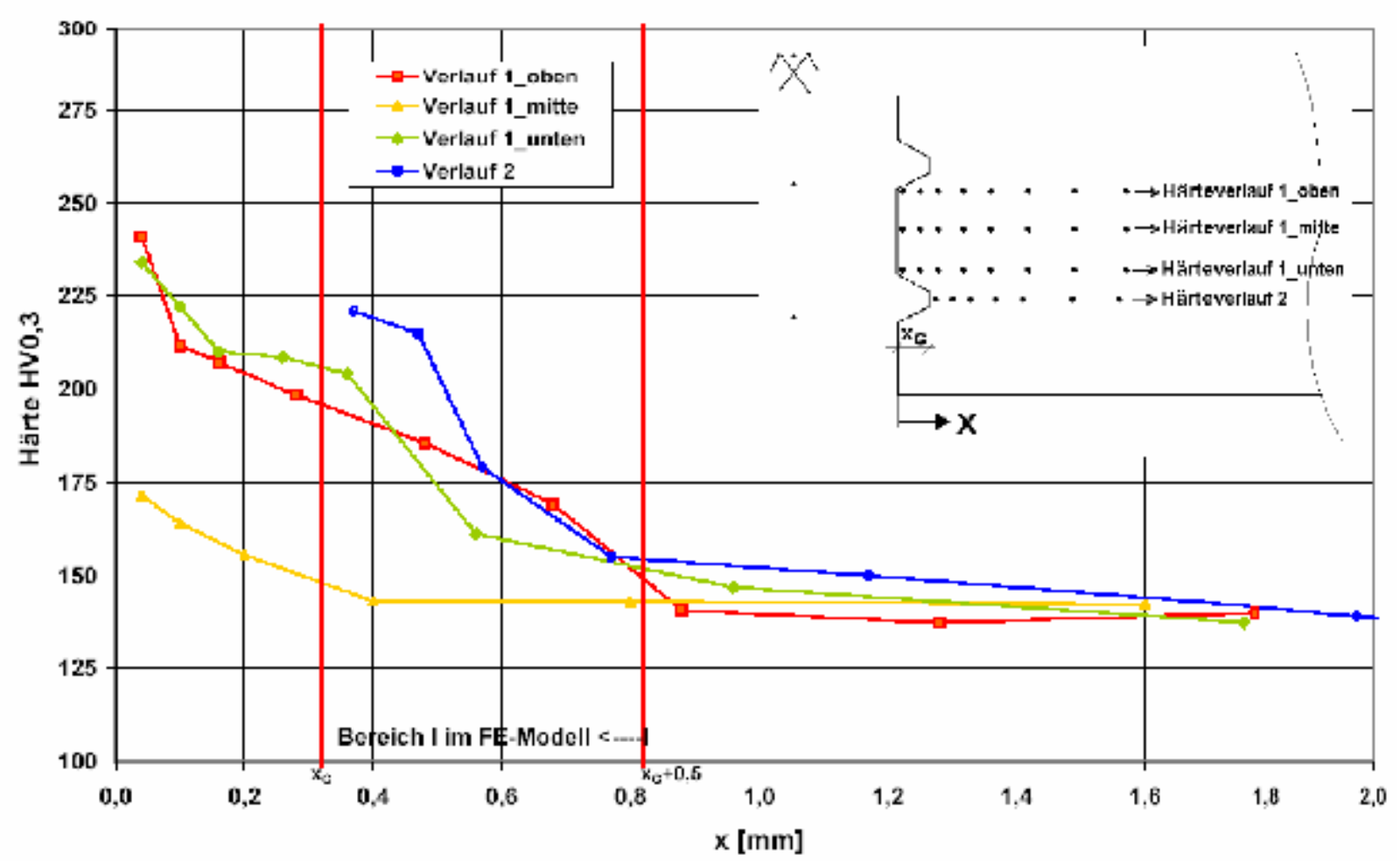

Diagramm 4.3: Härteprüfungen in der Unterkonstruktion Nr. 100=400S nach dem Gewindeformvorgang mit der Schraube Nr. 61=C=Cvc 
Der Einfluß einer im Muttergewinde aus der Kaltverfestigung vorliegenden erhöhten Werkstoffestigkeit auf die Auszugstragfähigkeit wurde in den FE-Berechnungen folgendermaßen untersucht:

- Für die Unterkonstruktionen Nr. 100=400S und Nr. 94=63S wurden zusätzlich zu den Berechnungen, in denen das Werkstoffverhalten der gesamten Unterkonstruktion über die aus den einaxialen Zugversuchen abgeleiteten Werkstoffgesetze beschrieben wurde (Diagramme B.1 und B.7), FE-Berechnungen durchgeführt, in denen für die Elemente im Bereich I des FE-Modells Werkstoffgesetze verwendet wurden, die gegenüber den jeweiligen aus den einaxialen Zugversuchen ermittelten Werkstoffgesetze erhöhte Festigkeiten aufweisen (Diagramme B.13 und B.14).

- Zur Festlegung der Werkstoffgesetze mit den erhöhten Festigkeiten, wurde ein für den gesamten Bereich I repräsentativer mittlerer Härtewert aus den in den Diagrammen 4.2 und 4.3 dargestellten Härteverläufe ermittelt, indem diese Härteverläufe zunächst durch jeweils einen in vertikaler Richtung konstant angenommenen mittleren Härteverlauf angenähert wurden, der in radialer Richtung über den Bereich I linear abfällt. Dieser angenäherte Härteverlauf ergibt sich, indem für die Stützstelle $x=x_{G}+0,5 \mathrm{~mm}$ ein Härtewert von 145HV0,3 angenommen wurde und für die Stützstelle direkt an der Oberfläche ( $x=0,04 \mathrm{~mm})$ der Härtewert ermittelt wurde, indem an dieser Stelle der Mittelwert aus den Härtewerten der Verläufe 1_oben und 1_unten mit dem Härtewert des Verlaufs 1_mitte gemittelt wurde. Dieses Vorgehen liefert für die Stützstelle direkt an der Oberfläche für die Annäherung der Härteverläufe im Diagramm 4.2 einen Härtewert von 218HV0,3 und für die Annäherung der Härteverlaufe aus Diagramm 4.3 einen Härtewert von 202HV0,3. Der für den Bereich I repräsentative Härtewert wurde dann aus den angenäherten Härteverläufen ermittelt, indem der Mittelwert der Härtewerte an den Stützstellen gebildet wurde (mittlere Härte des Bereichs $\left.I=1 / 4^{*}\left(218 H V 0,3+202 H V 0,3+2^{*} 145 H V 0,3\right)=177 H V 0,3\right)$. Dieser mittlere Härtewert von 177HV0,3 liegt etwa $20 \%$ über dem Härtewert von $145 \mathrm{HV} 0,3$, der in etwa repräsentativ ist für den nicht verfestigten Bereich der Unterkonstruktion.

- Da die mittlere Härte des Bereichs I um ca. 20\% größer ist als die Härte des nicht verfestigten Bereichs der Unterkonstruktion, und da gemäß DIN EN ISO 18265 zwischen den Zugfestigkeiten und den Vickershärten für unlegierte und niedriglegierte Stähle eine annähernd lineare Beziehung besteht, wurden für die verfestigten Werkstoffgesetze Zugfestigkeiten angenommen, die 20\% über den Zugfestigkeiten der aus den einaxialen Zugversuchen abgeleiteten Werkstoffgesetze liegen.

- Da mit der Verfestigung auch eine Abnahme der Verformungsmöglichkeit für den Werkstoff auftritt, wurden die wahren Spannungs-Dehnungsbeziehungen der Werkstoffgesetze 
mit der erhöhten Festigkeit aus den jeweiligen über die einaxialen Zugversuche ermittelten wahren Spannungs-Dehnungsbeziehungen abgeleitet, indem für alle im plastischen Bereich liegenden Spannungs-Dehnungspunkte die Spannungswerte mit dem Faktor 1,2 multipliziert wurden, während gleichzeitig die Dehnungswerte durch 1,2 dividiert wurden.

- Die FE-Berechnungen, in denen für die Elemente im Bereich I die Werkstoffgesetze mit der erhöhten Festigkeit verwendet wurden, sind mit den Bezeichnungen „63S_v“ und „400S_v“ gekennzeichnet.

Für Schrauben aus nichtrostendem, austenitischem Stahl wurde zur Beschreibung der technischen Spannungs-Dehnungsbeziehung das in [37] für nichtrostende Stähle angegebene Potenzgesetz (4.3) verwendet.

$\varepsilon=\frac{\sigma}{E}+0,002\left[\frac{\sigma}{R_{p 0,2}}\right]^{n} \quad$ mit: $\mathrm{n}=6$ für $\sigma \leq \mathrm{R}_{\mathrm{p} 0,2}$ und $\mathrm{n}=17$ für $\sigma>\mathrm{R}_{\mathrm{p} 0,2}$

Für den Elastizitätsmodul E wurde ein Wert von $190000 \mathrm{~N} / \mathrm{mm}^{2}$ angenommen.

Den weiteren notwendigen Annahmen für die 0,2\%-Dehngrenze $R_{p 0,2}$ und für die Zugfestigkeit $\mathrm{R}_{\mathrm{m}}$ liegen folgende Überlegungen zugrunde:

- Die Abschätzungen in der Tabelle A.2 liefern für die Zugfestigkeiten des Schraubenkernquerschnittes im Gewindebereich für alle ausgewerteten nichtrostenden Schrauben einen Mittelwert von $930 \mathrm{~N} / \mathrm{mm}^{2}$ (vgl. Tabelle 3.1, Kapitel 3) und speziell für die nichtrostenden Schrauben, die zur Verifikation des FE-Modells ausgewählt wurden, einen Mittelwert von $955 \mathrm{~N} / \mathrm{mm}^{2}$ (vgl. Tabelle 4.2, Abschnitt 4.2).

- Zur Untersuchung der Fragestellung, ob in den Gewindegängen von nichtrostenden Schrauben im Vergleich zum Schraubenkernquerschnitt infolge des Herstellvorganges größere Festigkeiten vorliegen, wurden Kleinlasthärteprüfungen an Schnitten durch die Gewindebereiche der Schrauben Nr. 67=A und Nr. 61=C=Cvc der Tabelle 4.2 durchgeführt, deren Ergebnisse in den Diagrammen 4.4 und 4.5 dargestellt sind.

- Die Ergebnisse dieser Härteprüfungen zeigen, daß die oberflächennahen Gewindebereiche sowohl in den Gewindegängen (Härteverlauf 1) als auch im Schraubenkernquerschnitt (Härteverlauf 2) erhöhte Festigkeiten aufweisen. Die größten Härtewerte ergeben sich für beide Schrauben an der Oberfläche des Schraubenkerns (Härteverlauf 2). Für die Schraube Nr. 67=A zeigt der Vergleich der beiden Härteverläufe 1 und 2, daß in der ersten Hälfte des Schraubenkernquerschnitts Festigkeiten vorliegen, die etwa in der gleichen Größenordnung liegen wie die Festigkeiten in den Gewindegängen. Die Härteverläufe bei der Schraube Nr. 61=C=Cvc lassen sogar auf eine im Vergleich zum Kernquerschnitt etwas kleinere Festigkeit der Gewindegänge schließen. 
- Aufgrund der Ergebnisse der Härteprüfungen wurden die Abschätzungen für die Zugfestigkeiten des Schraubenkernquerschnittes im Gewindebereich auch als eine geeignete Annäherung für die Zugfestigkeit der Gewindegänge von nichtrostenden Schrauben angesehen.

- Unter Berücksichtigung der festigkeitssteigernden Kerbwirkung des Gewindes, aufgrund derer die tatsächlichen Zugfestigkeiten des Schraubengewindes durch die abgeschätzten Zugfestigkeiten um bis zu 10\% überschätzt werden (vgl. Abschnitt 2.2.2), wurde eine Zugfestigkeit von $850 \mathrm{~N} / \mathrm{mm}^{2}$ zur Beschreibung des Werkstoffverhaltens des Gewindes von nichtrostenden Schrauben angenommen.

- Diese Zugfestigkeit von $850 \mathrm{~N} / \mathrm{mm}^{2}$ liegt innerhalb des Bereichs von $800 \mathrm{~N} / \mathrm{mm}^{2}$ bis $1000 \mathrm{~N} / \mathrm{mm}^{2}$, der in DIN 17440 für die Zugfestigkeiten von kaltverfestigten Drähten aus austenitischem Stahl für die Verfestigungsstufe C800 angegeben wird. Zur Beschreibung des Werkstoffverhaltens des Gewindes von nichtrostenden Schrauben über die Beziehung (4.3) wurde daher zusätzlich zu der abgeschätzten Zugfestigkeit von $850 \mathrm{~N} / \mathrm{mm}^{2}$ der Wert für die 0,2\%-Dehngrenze mit $650 \mathrm{~N} / \mathrm{mm}^{2}$ so angenommen, daß die in DIN 17440 angegebenen Grenzwerte der Verfestigungsstufe C800 für die 0,2\%-Dehngrenzen $\left(500 \mathrm{~N} / \mathrm{mm}^{2}\right.$ bis $\left.750 \mathrm{~N} / \mathrm{mm}^{2}\right)$ ebenfalls eingehalten wurden.

- Das entsprechende Werkstoffgesetz wurde in den FE-Berechnungen mit „NRH“ bezeichnet und ist im Diagramm B.15 dargestellt.

- Als eine untere Abschätzung für die Spannungs-Dehnungsbeziehung des Gewindes von nichtrostenden Schrauben wurde das im Diagramm B.16 dargestellte Werkstoffgesetz verwendet, welches aus der Beziehung (4.3) und den Werten $E=190000 \mathrm{~N} / \mathrm{mm}^{2}$, $R_{m}=680 \mathrm{~N} / \mathrm{mm}^{2}$ und $R_{p 0,2}=510 \mathrm{~N} / \mathrm{mm}^{2}$ resultiert. Dieses Werkstoffgesetz paßt in etwa zu den Angaben in DIN 17440 für die mechanischen Eigenschaften eines kaltverfestigten, austenitischen Stahls der Verfestigungsstufe C700. Dieses Werkstoffgesetz wurde in den FE-Berechnungen mit „NRL“ bezeichnet.

- Für die in der Tabelle A.2 aufgeführten nichtrostenden Schrauben wurde für die maximale Zugfestigkeit des Schraubenkernquerschnitts ein Wert von $1299 \mathrm{~N} / \mathrm{mm}^{2}$ abgeschätzt, der sogar noch über dem Mittelwert der für Schrauben aus niedriglegierten Kohlenstoffstählen abgeschätzten Zugfestigkeiten liegt (vgl. Tabelle 3.1, Kapitel 3). Die Untersuchung der Auszugstragfähigkeit von Schrauben, die Zugfestigkeiten in dieser Größenordnung aufweisen, ist daher durch die FE-Berechnungen abgedeckt, die mit den für Schrauben aus niedriglegierten Kohlenstoffstählen entwickelten Werkstoffgesetzen durchgeführt wurden. 


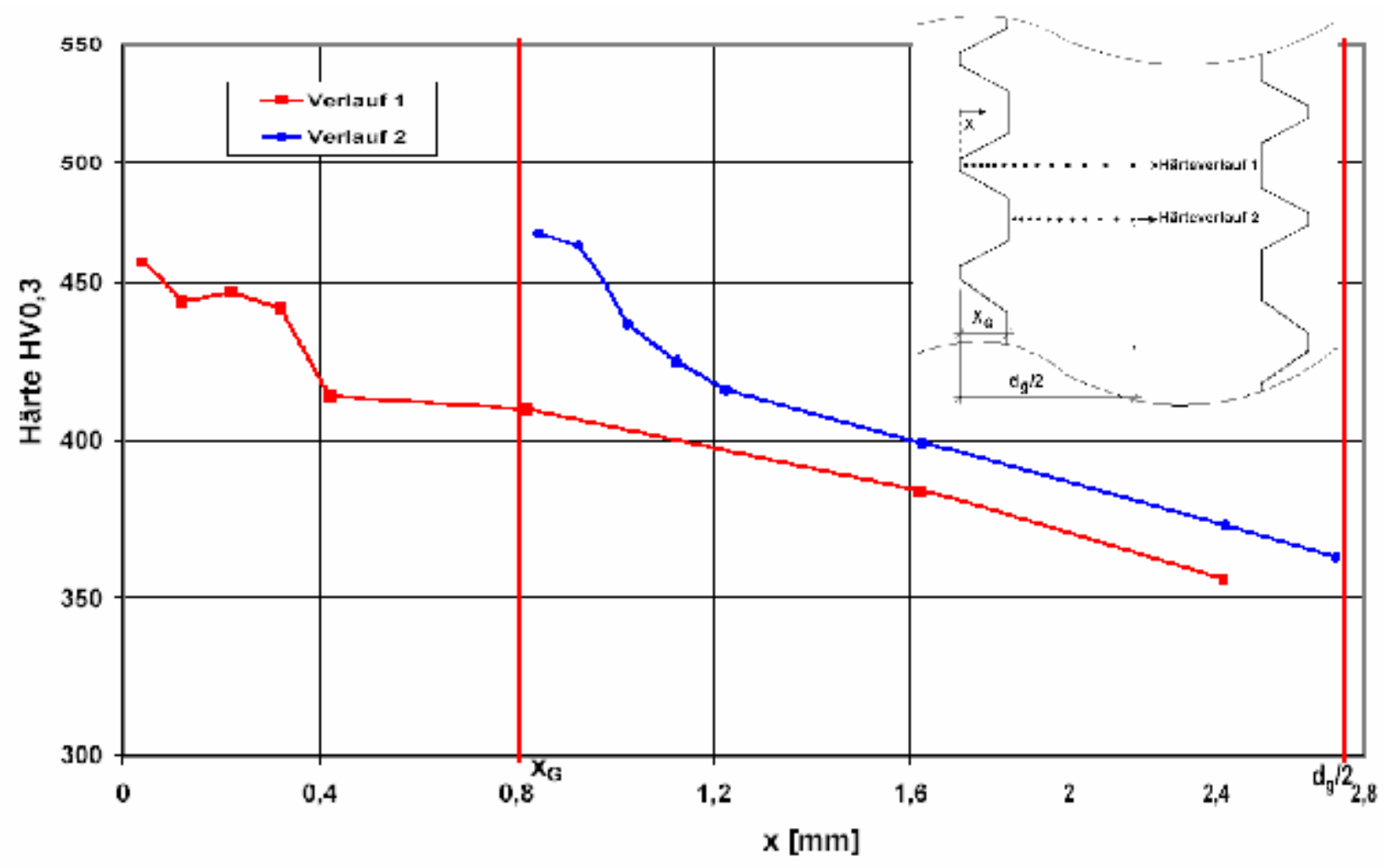

Diagramm 4.4: Härteprüfungen im Gewindebereich der Schraube Nr. 67=A

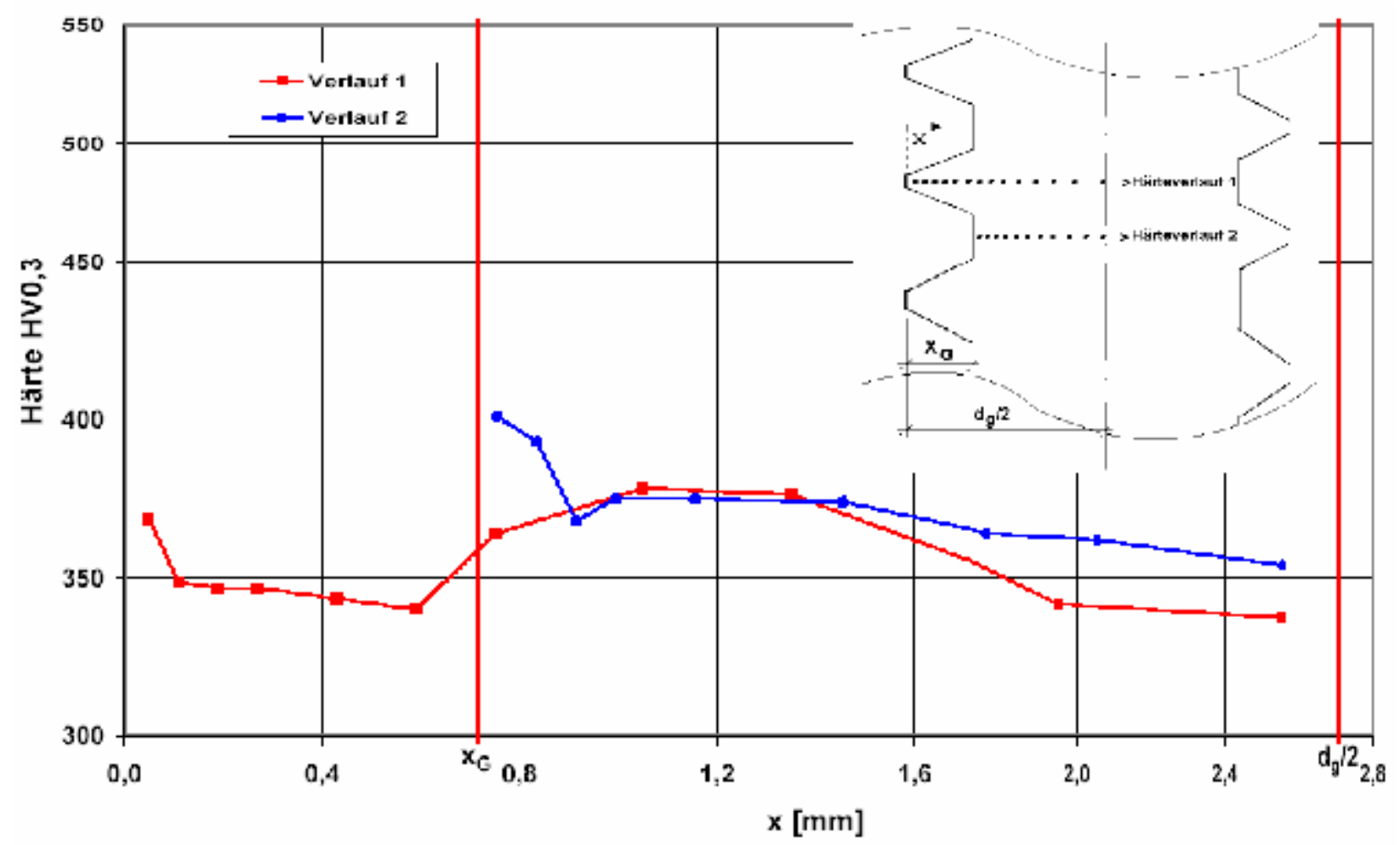

Diagramm 4.5: Härteprüfungen im Gewindebereich der Schraube Nr. 61=C=Cvc In den Diagrammen B.17 bis B.19 sind die Werkstoffgesetze dargestellt, die zur Beschreibung des Werkstoffverhaltens der Gewindegänge von Schrauben aus niedriglegierten Kohlenstoffstählen verwendet wurden. 
Die im Diagramm B.17 angegebene technische Spannungs-Dehnungsbeziehung wurde aus [38] entnommen. Sie wurde dort verwendet zur Beschreibung des Werkstoffverhaltens einer Schraube der Festigkeitsklasse 10.9 gemäß DIN EN ISO 898-1. Die zugrundegelegten Werte für die Zugfestigkeit $R_{m}=1040 \mathrm{~N} / \mathrm{mm}^{2}$ und für die $0,2 \%$-Dehngrenze $R_{p 0,2}=940 \mathrm{~N} / \mathrm{mm}^{2}$ entsprechen den in DIN EN ISO 898-1 für die Festigkeitsklasse 10.9 festgelegten Mindestwerten. Dieses Werkstoffgesetz wurde in den FE-Berechnungen mit „CL“ bezeichnet. Die Auswertungen in der Tabelle $3.1 \mathrm{im}$ Kapitel 3 liefern für Schrauben aus niedriglegierten Kohlenstoffstählen als Abschätzung für die Zugfestigkeiten des Schraubenkernquerschnittes im Gewindebereich einen Mittelwert von $1190 \mathrm{~N} / \mathrm{mm}^{2}$ und speziell für die Schraube Nr. 66=J, die zur Verifikation des FE-Modells ausgewählt wurde, einen Wert von $1212 \mathrm{~N} / \mathrm{mm}^{2}$ (vgl. Tabelle 4.2, Abschnitt 4.2). Unter Berücksichtigung der festigkeitssteigernden Kerbwirkung des Gewindes kann daher das Werkstoffgesetzt „CL“ als eine Abschätzung betrachtet werden, die für die Gewindegänge der Schraube etwa die im Gewindekernquerschnitt vorliegenden Festigkeitsverhältnisse unterstellt.

Eine Untersuchung der sich aus der Wärmebehandlung ergebenden unterschiedlichen Festigkeitsverhältnisse im Gewinde von Schrauben aus niedriglegierten Kohlenstoffstählen erfolgte anhand von Kleinlasthärteprüfungen, die an einem Schnitt durch das Gewinde der Schraube Nr. 66=J durchgeführt wurden. Die in dem Diagramm 4.6 dargestellten Ergebnisse dieser Härteprüfungen verdeutlichen folgendes:

- An der Oberfläche des Schraubengewindes liegen Härtewerte vor, die um bis zu 70\% über den Härtewerten liegen, die für den überwiegenden Bereich des Schraubenkerns gemessen wurden. Die größten Härtewerte treten an der Oberfläche der Gewindegänge auf (Härteverlauf 1).

- Die extremen Härtewerte klingen mit zunehmendem Oberflächenabstand sehr rasch ab und erreichen in einem Abstand von ca. 0,2mm sowohl in den Gewindegängen (Härteverlauf 1) als auch im Schraubenkern (Härteverlauf 2) ein horizontales Plateau. Die Härtewerte dieser horizontalen Plateaus sind in den Gewindegängen um bis zu 14\% größer als im Schraubenkernquerschnitt. 


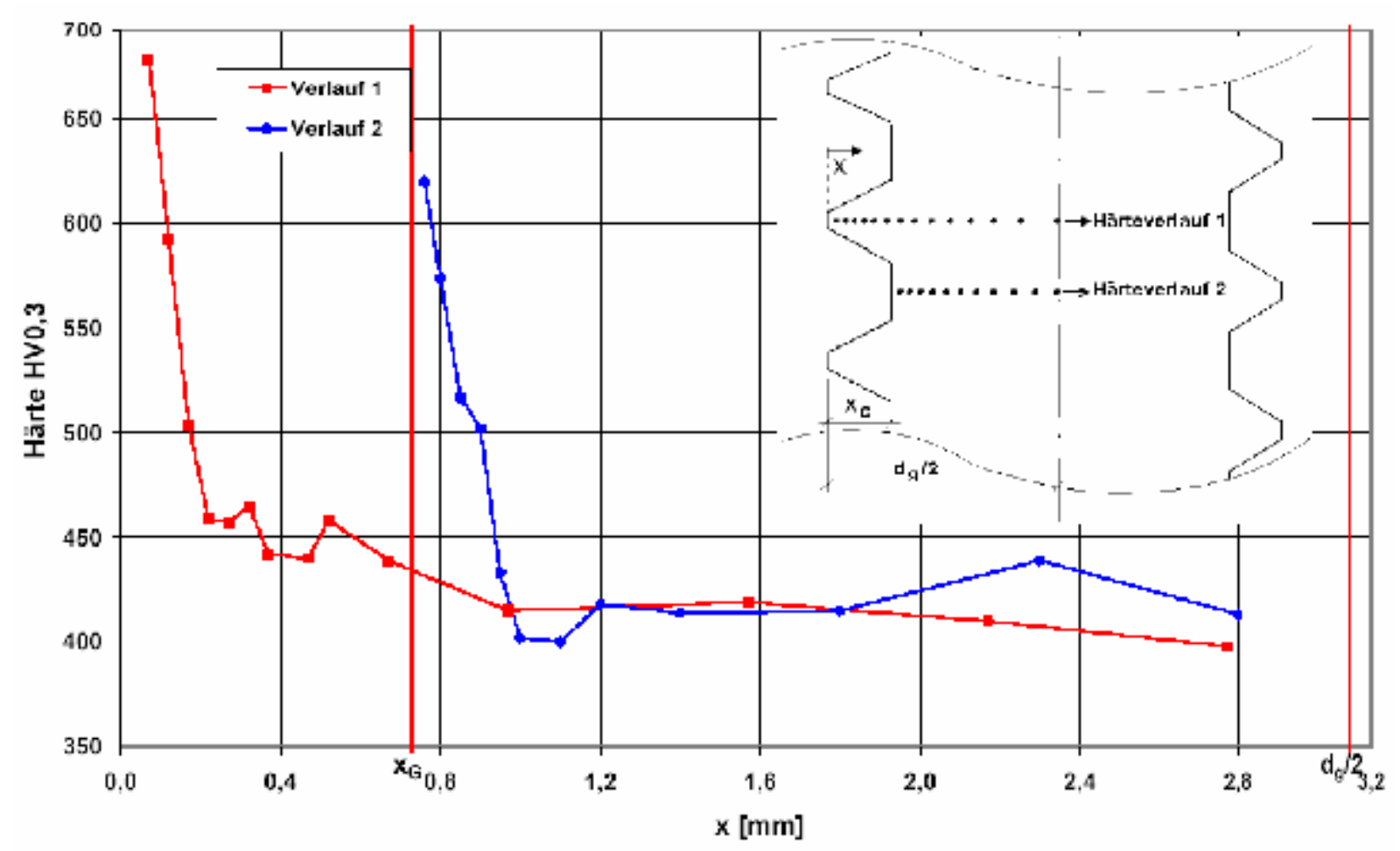

Diagramm 4.6: Härteprüfungen im Gewindebereich der Schraube Nr. 66=J

Den Ergebnissen der Härteprüfungen wurde in den FE-Berechnungen folgendermaßen Rechnung getragen:

- Zur Berücksichtigung einer im Vergleich zum Schraubenkern größeren Festigkeit in den Gewindegängen der Schraube wurde das im Diagramm B.18 dargestellte Werkstoffgesetz verwendet, das eine Zugfestigkeit von $R_{m}=1250 \mathrm{~N} / \mathrm{mm}^{2}$ und eine 0,2\%-Dehngrenze von $R_{p 0,2}=1130 \mathrm{~N} / \mathrm{mm}^{2}$ unterstellt. Dieses Werkstoffgesetz wurde in den FEBerechnungen mit „ $\mathrm{CH}^{\prime}$ bezeichnet.

- Zur Berücksichtigung der größeren Festigkeiten, die auf den oberflächennahen Randbereichen der Gewindegänge vorliegen, und zur Berücksichtigung der aus der Verfestigung auftretenden abnehmenden Verformungsmöglichkeit für den Werkstoff wurde aus dem Werkstoffgesetz „CH“ das Werkstoffgesetz "CHR“ im Diagramm B.19 entwickelt. Zur Festlegung dieses Werkstoffgesetzes wurde analog vorgegangen wie bei der Festlegung der Werkstoffgesetze für den verfestigten Bereich I der Unterkonstruktion. Aus dem im Diagramm 4.6 dargestellten Härteverlauf 1 ergibt sich für die oberflächennahen Randbereiche der Gewindegänge ein mittlerer, repräsentativer Härtewert von $569 H V 0,3=1 / 2 *(680 H V 0,3+459 H V 0,3)$, der etwa $20 \%$ über dem größten Härtewert von $465 \mathrm{HV} 0,3$ liegt, der auf dem restlichen Bereich der Gewindegänge auftritt. Das Werkstoffgesetz "CHR“ wurde daher aus dem Werkstoffgesetz "CH“ entwickelt, indem bei der wahren Spannungs-Dehnungsbeziehung des Werkstoffgesetzes „CH“ für alle im plastischen Bereich liegenden Spannungs-Dehnungspunkte die Spannungswerte mit dem Faktor 1,2 multipliziert wurden während gleichzeitig die Dehnungswerte durch 1,2 divi- 
diert wurden. Das Werkstoffgesetz "CHR“ wurde in den FE-Berechnungen für die Schraube „J“ ausschließlich auf diejenigen Elemente angewendet, die den äußersten radialen Ring der Gewindegänge bilden (vgl. Tabelle 4.4 und Bild 4.2 im Abschnitt 4.4.1). Dies bedeutet, für die Schraube „J“ wurde das Werkstoffgesetz „CHR“ zur Beschreibung des Werkstoffverhaltens derjenigen Elemente verwendet, die innerhalb eines radialen Abstandes von $0,15 \mathrm{~mm}$ zum Gewindeaußendurchmesser liegen. Für die restlichen Elemente der Gewindegänge wurde das Werkstoffgesetzt " $\mathrm{CH}^{\prime \prime}$ verwendet. Die entsprechenden FE-Berechnungen sind durch die Doppelbezeichnung „CH_CHR“ gekennzeichnet.

\subsection{Kontaktmodellierung}

Der Kontakt zwischen den Gewindeflanken der Schraube und dem Muttergewinde wurde durch Kontaktelemente CONTA173 und TARGE170 abgebildet.

Durch eine entsprechende Anordnung der Kontaktelemente auf den Flankenoberflächen des Schrauben- und Muttergewindes sowie auf der Blechunterseite der Unterkonstruktion können Kontaktkräfte

- zwischen dem Schraubengewinde und dem Muttergewinde auf den Oberseiten ihrer Gewindeflanken sowie

- im Auslauf des Muttergewindes, zwischen der Unterseite der Unterkonstruktion sowie der Oberseite der Gewindeflanken der Schrauben

übertragen werden.

Im Bild 4.8 sind die möglichen Kontaktzonen für den Fall, daß der Durchmesser des Kernloches $d_{L}$ in der Unterkonstruktion größer als der Kerndurchmesser $d_{K}$ des Schraubengewindes ist (Schrauben „A“, „C“, „Cvc“, „E“, „F2“, „G“, „H“, „l“ und „J“ in Tabelle 4.2) rot gekennzeichnet.

Die CONTA173 Elemente wurden auf die Oberseite der Mutterflanken sowie, im Bereich des Auslauf des Muttergewindes, auf die Unterseite der Unterkonstruktion gelegt. Die TARGE170 Elemente wurden auf der Oberseite der Gewindeflanken der Schrauben angeordnet.

Für den Fall $d_{L}=d_{K}$ (Schrauben „B“, „D“ und „F1“ in Tabelle 4.2) wurden ebenfalls die oben beschriebenen Kontaktzonen im FE-Modell abgebildet (Bild 4.9). Die für diesen Fall bestehende Möglichkeit eines horizontalen Kontaktes zwischen dem Schraubenschaft und der Unterkonstruktion wurde aus folgenden Gründen nicht modelliert: 
- Sofern es zu einem horizontalen Kontakt zwischen dem Schraubenschaft und der Unterkonstruktion kommt, können aus diesem Kontakt in Richtung der Schraubenachse lediglich Reibungskräfte übertragen werden. Diese Reibungskräfte werden gegenüber den Vertikalkomponenten der auftretenden Kontaktkräfte zwischen den Gewindeflanken der Schraube und der Mutter als untergeordnet angesehen.

- Gemäß [29], [39] bis [43] tritt bei zugbeanspruchten metrischen Schrauben-MutternVerbindungen eine radiale Aufweitung der Mutter auf, die durch die Radialkomponenten der auf die Gewindeflanken der Mutter wirkenden Kontaktkräfte hervorgerufen wird. Dieser Effekt rechtfertigt die Vernachlässigung des horizontalen Kontaktes zwischen dem Schraubenschaft und der Unterkonstruktion im FE-Modell.

- Zusätzlich zu der radialen Mutteraufweitung führt auch die durch die Zugbeanspruchung der Schraube hervorgerufene Querkontraktion im Schraubenschaft dazu, daß ein horizontaler Kontakt zwischen dem Schraubenschaft und der Unterkonstruktion nicht auftritt.

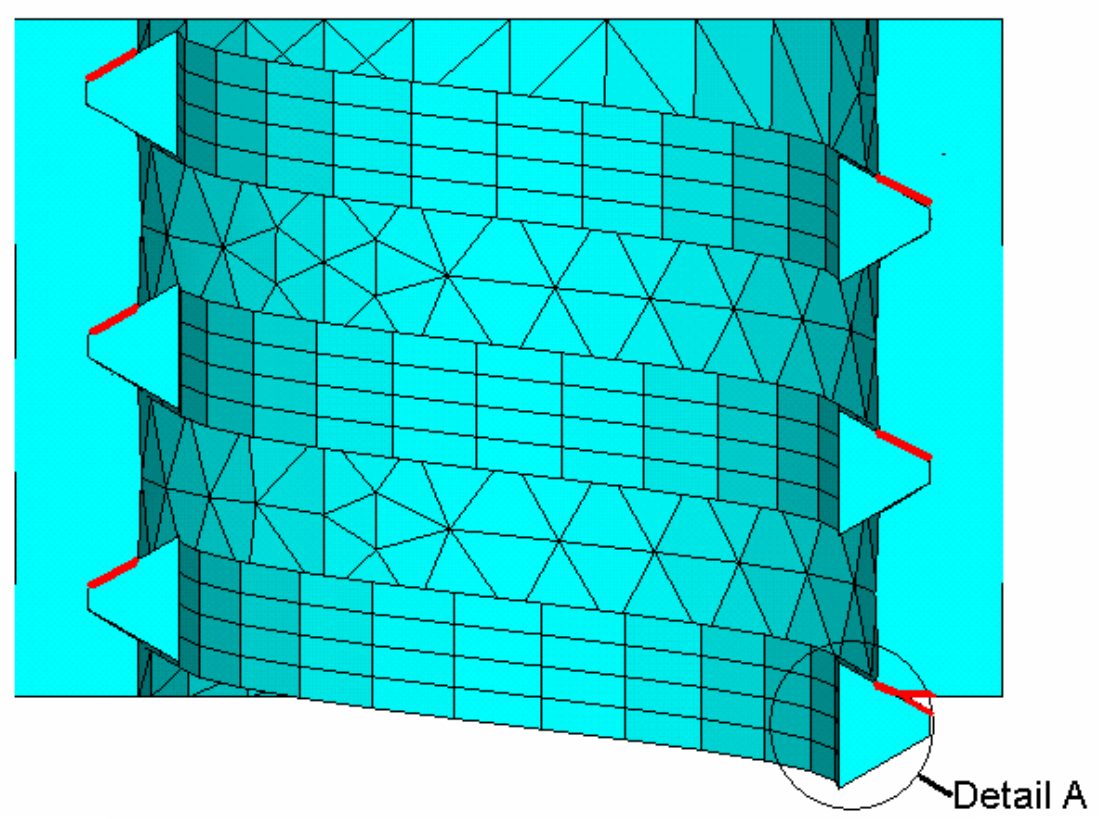

Detall A: Konlaktmāglichkøit im Gewindeauslaur des Mutrergevilndes

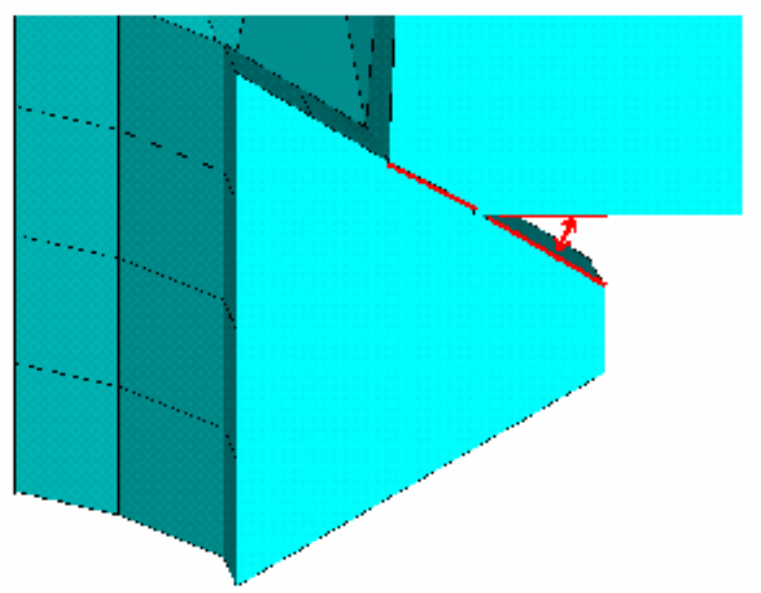

Bild 4.8: Mögliche Kontaktzonen für den Fall $d_{L}>d_{K}$ 


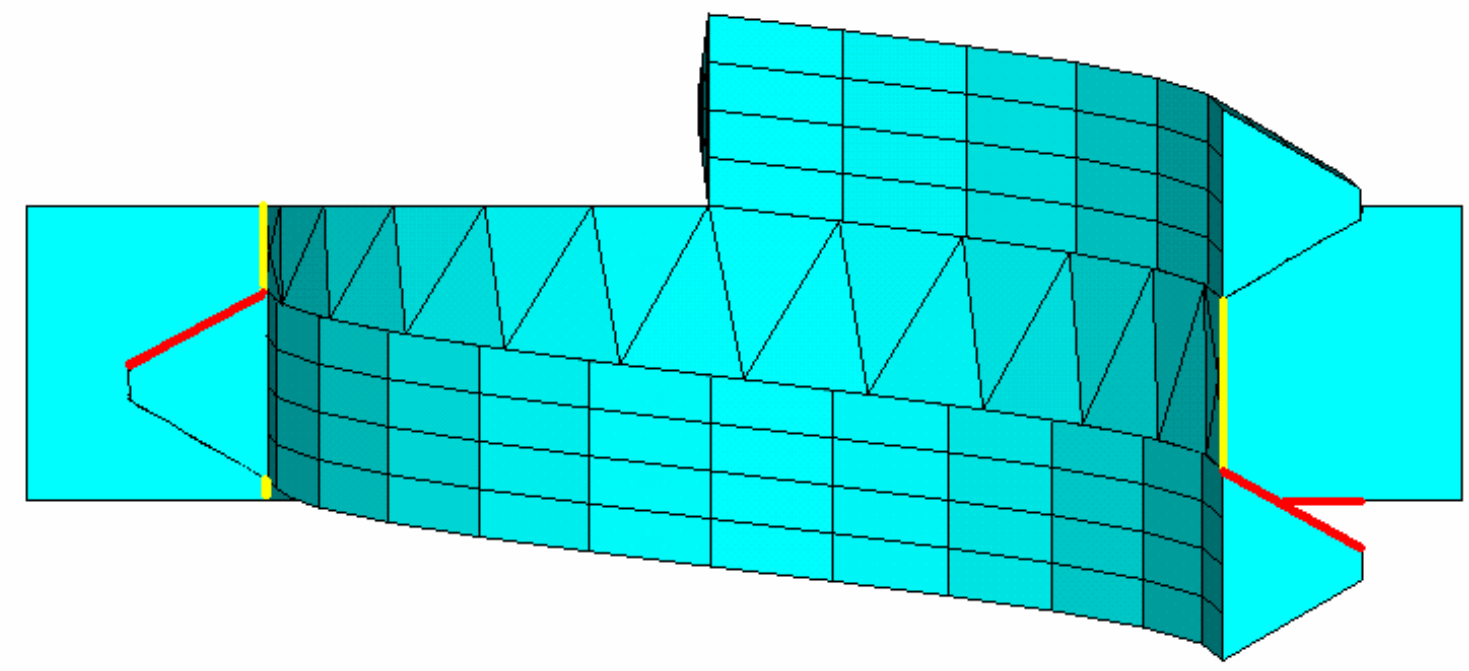

im FE-Modell abgebildete Kontaktzonen

\section{horizontale Kontaktmöglichkeit, die im FE-Modell nicht abgebildet wurde}

Bild 4.9: Mögliche Kontaktzonen für den Fall $d_{L}=d_{K}$

Zur Abbildung der Reibung wurden folgende Reibungskoeffizienten für die berücksichtigten Kontaktzonen angenommen:

- $\quad$ In [29] werden für die Paarung von metrischen Schrauben aus nichtrostendem, austenitischem Stahl mit Muttern aus der Aluminiumlegierung AIMgSi Gewindereibungskoeffizienten im Bereich von 0,28 bis 0,43 angegeben. Der Grund für diese großen Reibungskoeffizienten liegt nach [29] in erster Linie in der für austenitische Schrauben charakteristischen großen Werkstoffzähigkeit. Aufgrund dieses Sachverhaltes wurden in allen FEBerechnungen, in denen das Werkstoffverhalten einer austenitischen Schraube abgebildet wurde, ein Reibungskoeffizient von $\mu_{G}=0,35$ für die Gewindereibung zwischen den Schrauben- und Mutterflanken angenommen.

- Für metrische Schrauben aus niedriglegierten Kohlenstoffstählen werden in [29] und [44] Gewindereibungskoeffizienten angegeben, die im Bereich zwischen 0,10 und 0,30 liegen. Zur Untersuchung des Einflusses unterschiedlicher Reibungskoeffizienten wurden die FE-Berechnungen einmal mit dem für austenitische Schrauben angenommenen Reibungskoeffizient von $\mu_{\mathrm{G}}=0,35$ und ein zweites mal mit dem in [29] und [44] angegebenen unteren Grenzwert von $\mu_{\mathrm{G}}=0,10$ durchgeführt.

- Für den möglichen Kontaktbereich zwischen den Gewindeflanken der Schrauben und der Blechunterseite im Gewindeauslauf des Muttergewindes wurde in allen FEBerechnungen ein Reibungskoeffizient von $\mu=0,10$ angenommen. 


\subsection{Lagerungsbedingungen}

Die Auflagerung der Unterkonstruktion erfolgte an ihren Querrändern über die Knoten, die auf der Gurtoberseite der Unterkonstruktion liegen.

Zur Abbildung der in den Auszugversuchen vorliegenden Randbedingungen (Bild 2.14, Abschnitt 2.3) erfolgte die Auflagerung in z-Richtung (rechtwinklig zur Gurtfläche) über die Kontaktelemente CONTA52. Damit wurde sichergestellt, daß an den Querrändern der Unterkonstruktion keine Zugkräfte in z-Richtung auftreten können. Für die Gesamtsteifigkeit der Kontaktelemente an einem Querrand wurde eine Steifigkeit von $100 \mathrm{kN} / \mathrm{mm}$ angenommen - dies bedeutet, daß die Auflagervorrichtung an der Stelle eines Querrands mit 100kN belastet werden müßte, damit sie sich um $1 \mathrm{~mm}$ vertikal zu diesem Querrand verschiebt. Diese Gesamtsteifigkeit wurde auf die Kontaktelemente im Verhältnis ihrer Einflußlängen am Querrand aufgeteilt.

In x und y Richtung (in der Gurtebene) wurden die auf der Gurtoberseite der Querränder liegenden Elementknoten starr gelagert.

Die auf dem Kerndurchmesser $d_{k}$ liegenden Knoten der Gewindegänge der Schraube wurden in radialer Richtung und in Umfangsrichtung starr gelagert und in Richtung der Schraubenachse starr miteinander gekoppelt.

Die Last wurde in der FE-Berechnung aufgebracht, indem alle gelagerten Schraubenknoten um den gleichen Betrag in Richtung der Schraubenachse verschoben wurden.

\subsection{Ergebnisse der simulierten Versuchsserien}

\subsubsection{Versagenskriterien und Verformungsverhalten}

In den FE-Berechnungen traten beim Erreichen ihrer Maximallasten die nachfolgenden beschriebenen Grenzzustände für das Versagens- und Verformungsverhalten auf:

- In den Berechnungen, in denen die Blechdicke und die Zugfestigkeit der Unterkonstruktion groß ist und gleichzeitig die Zugfestigkeit der Gewindegänge der Schraube sowie die radiale Überdeckung der Gewindegänge der Schraube durch das Muttergewinde klein ist, wird das Versagen bestimmt durch ein Versagen der Gewindegänge der Schraube. Dieser Versagensmechanismus wird deutlich anhand der FE-Berchnung für die Schraube „A“, dem Werkstoffgesetz „NRL“ und der Unterkonstruktion „400S“ (Bilder 4.10 bis 4.13). In den Gewindegängen der Schraube treten die größten Beanspruchungen bei dem Radius auf, der dem Durchmesser $d_{L}$ des Kernloches der Unterkonstruktion entspricht. Die Größe der Vergleichsspannung in den Gewindegängen der Schraube erreicht in diesem Schnitt über nahezu die gesamte Blechdicke der Unterkonstruktion die Größenordnung 
der wahren Zugfestigkeit des Werkstoffgesetztes „NRL“ $\left(R_{m, w a h r, N R L}=863 \mathrm{~N} / \mathrm{mm}^{2}\right)$ (Bilder 4.10 a) und b)). In der Unterkonstruktion treten die größten Beanspruchungen in den Gewindegängen des Muttergewindes auf (Bilder 4.10 c) und d)). Die Vergleichsspannung liegt hier jedoch fast ausschließlich deutlich unter dem Wert der wahren Zugfestigkeit des Werkstoffgesetzes „400S“ $\left(R_{m, w a h r, 400 s}=472 \mathrm{~N} / \mathrm{mm}^{2}\right)$ (Bild $\left.4.10 \mathrm{c}\right)$ ). Die vertikale Verschiebung (Verschiebung rechtwinklig zur Gurtebene) der auf dem Kerndurchmesser $d_{k}$ liegenden Schraubenknoten beträgt beim Erreichen der Maximallast ca. 0,6mm (Bild 4.11 a)). Diese Verschiebung setzt sich zusammen aus der Biegeverformung des Gurtes der Unterkonstruktion (ca. 0,4mm, Bild 4.11) sowie aus Verformungen in den Gewindegängen der Schraube (ca. 0,2mm), die sich aus der Differenz der in den Gewindegängen auftretenden Verschiebungen in den Schnitten $r=d_{K} / 2$ (ca. 0,6mm) und $r=d_{\llcorner} / 2$ (ca. $0,4 \mathrm{~mm}$ ) ergibt (Bild $4.11 \mathrm{a}$ )). Der zweitgenannte Verformungsanteil resultiert hauptsächlich aus großen plastischen Schubverzerrungen, die sich in den Gewindegängen der Schraube im Schnitt $r=d_{\llcorner} / 2$ einstellen (Bild $4.12 \mathrm{a}$ )). In den Gewindegängen des Muttergewindes treten überwiegend deutlich kleinere Schubverzerrungen auf (Bild 4.12 b)). Die Dehnungen in zirkularer Richtung, die in den Gewindegängen des Schrauben- und des Muttergewindes auftreten, liegen deutlich unter den jeweiligen Schubverzerrungen (Bilder 4.12 c) und d)). Die radialen Dehnungen in den Gewindegängen des Schrauben- und des Muttergewindes erreichen lediglich auf einem eng begrenzten Bereich an der Stelle, an der das Schraubengewinde in die Unterkonstruktion einläuft, die Größenordnungen der Schubverzerrungen (Bilder 4.12 e) und f)). Für den restlichen Bereich liegen die radialen Dehnungen in den Gewindegängen des Schrauben- und des Muttergewindes ebenfalls deutlich unter den jeweiligen Schubverzerrungen. Außerhalb des Muttergewindes (Bereich $r \geq d g / 2+0,5 \mathrm{~mm}$ ) bleiben sowohl die Dehnungen in radialer und zirkularer Richtung als auch die Schubverzerrungen in der Unterkonstruktion unterhalb 1,5\% (Bild 4.13).

- Mit abnehmender Blechdicke und Zugfestigkeit der Unterkonstruktion sowie mit zunehmender Zugfestigkeit der Gewindegänge der Schraube wird die in der FE-Berechnung erreichte Maximallast in zunehmenden Maße durch das Versagens- und Verformungsverhalten der Unterkonstruktion bestimmt. Der Versagensmechanismus, der durch ein Versagen der Unterkonstruktion bestimmt wird, ist beispielhaft für die Berechnung mit der Schraube „D“, dem Werkstoffgesetz „NRH“ und der Unterkonstruktion „63S“ in den Bildern 4.14 bis 4.17 dargestellt. Im Vergleich zu dem Versagensmechanismus, der durch die Gewindegänge der Schraube bestimmt wird, verlagern sich die maximalen Beanspruchungen, die in den Gewindegängen des Schrauben- und des Muttergewindes in der FEBerechnung D_NRH_63S auftreten, von dem Radius $r=d_{\llcorner} / 2$ hin zu dem Radius, der dem Gewindeaußendurchmesser $d_{g}$ der Schraube entspricht (Bild 4.14). In diesem Schnitt $r=d_{g} / 2$ erreichen die Vergleichsspannungen in den Gewindegängen des Muttergewindes 
nahezu über den gesamten Verzahnungsbereich zwischen Schrauben- und Muttergewinde die Größenordnung der wahren Zugfestigkeit des Werkstoffgesetzes „63S“ $\left(R_{m, w a h r, 63 s}=485 \mathrm{~N} / \mathrm{mm}^{2}\right)$ (Bild $\left.4.14 \mathrm{c}\right)$ ). Die Vergleichsspannungen in den Gewindegängen der Schraube bleiben größtenteils deutlich unter der wahren Zugfestigkeit des Werkstoff-

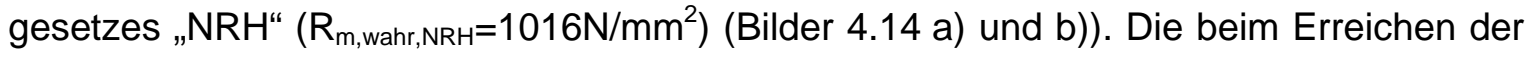
Maximallast auftretende vertikale Verschiebung der Gewindegänge der Schraube beträgt 4,7mm (Bild 4.15 a)). Diese Verschiebung resultiert hauptsächlich aus Biege- und Schubverformungen in der Unterkonstruktion (ca. 4,6mm, Bild 4.15) und zu einem geringen Anteil auch aus Relativverschiebungen, die zwischen den Gewindegängen der Schraube und den Gewindegängen des Muttergewindes auftreten (ca. 0,1 mm, Bild $4.11 \mathrm{a}$ )). In den Gewindegängen der Schraube treten große Schubverzerrungen und große radiale Dehnungen lediglich in der oberen Hälfte des Verzahnungsbereiches mit dem Muttergewinde auf (Bilder 4.16 a) und e)). Die zirkulare Dehnungen in den Gewindegängen der Schraube bleiben klein und erreichen nur punktuell einen maximalen Wert von 3,2\% (Bild 4.16 c)). In den Gewindegängen des Muttergewindes treten im gesamten Verzahnungsbereich mit der Schraube große Schubverzerrungen und große zirkulare Dehnungen auf (Bild 4.16 b) und d)). Die radialen Dehnungen im Muttergewinde bleiben dagegen klein und erreichen lediglich auf einem eng begrenzten Bereich an der Stelle, an der das Schraubengewinde in die Unterkonstruktion einläuft, die Größenordnung der Schubverzerrungen und der zirkularen Dehnungen (Bild $4.16 \mathrm{f}$ )). Die im Muttergewinde auftretenden großen Schubverzerrungen und Dehnungen zeigen mit zunehmenden radialen Abstand von der Schraubenachse ein sehr schnelles Abklingverhalten (Bild 4.17). 


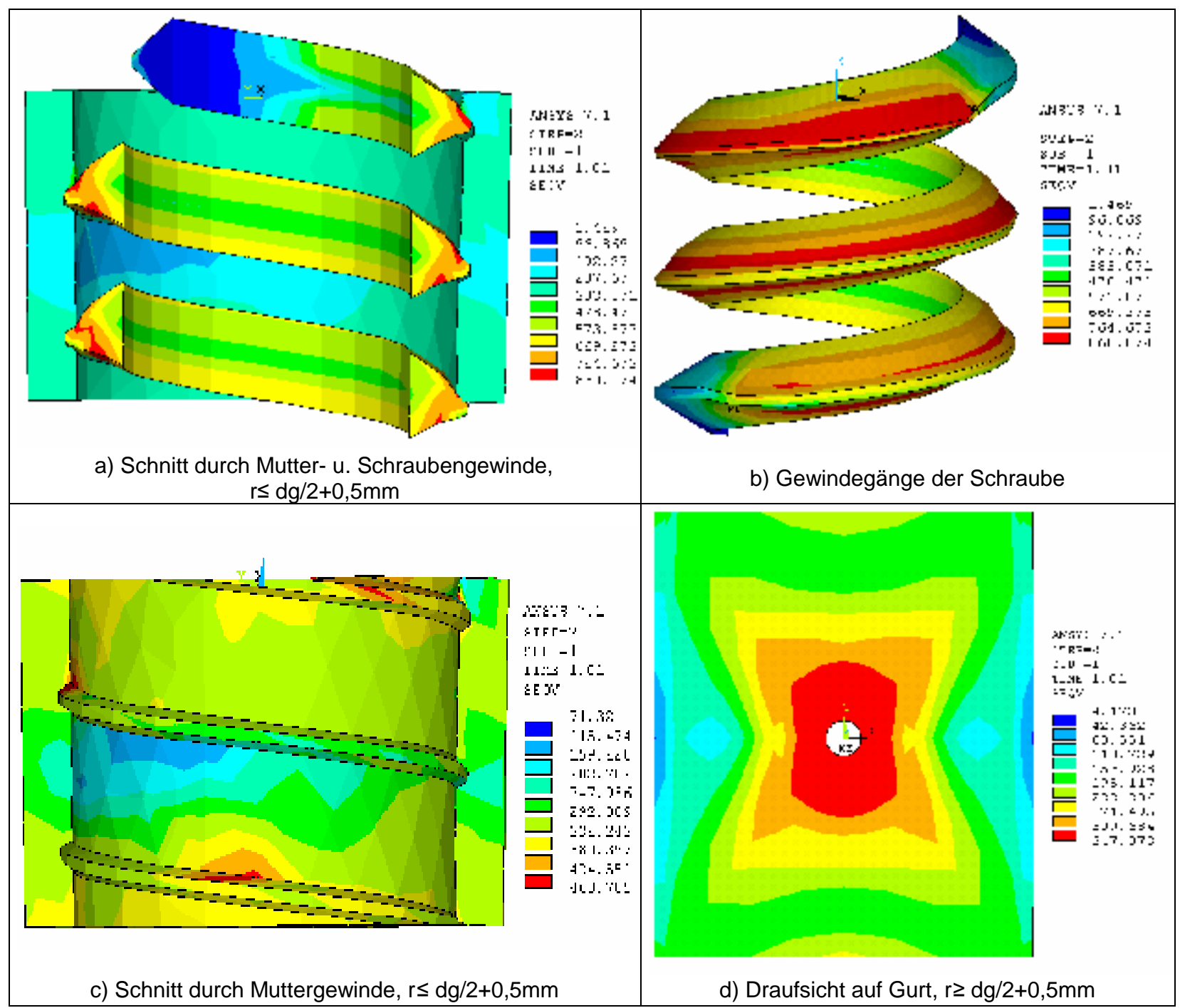

Bild 4.10: FE-Berechnung A_NRL_400S, Vergleichsspannung beim Erreichen der Maximallast

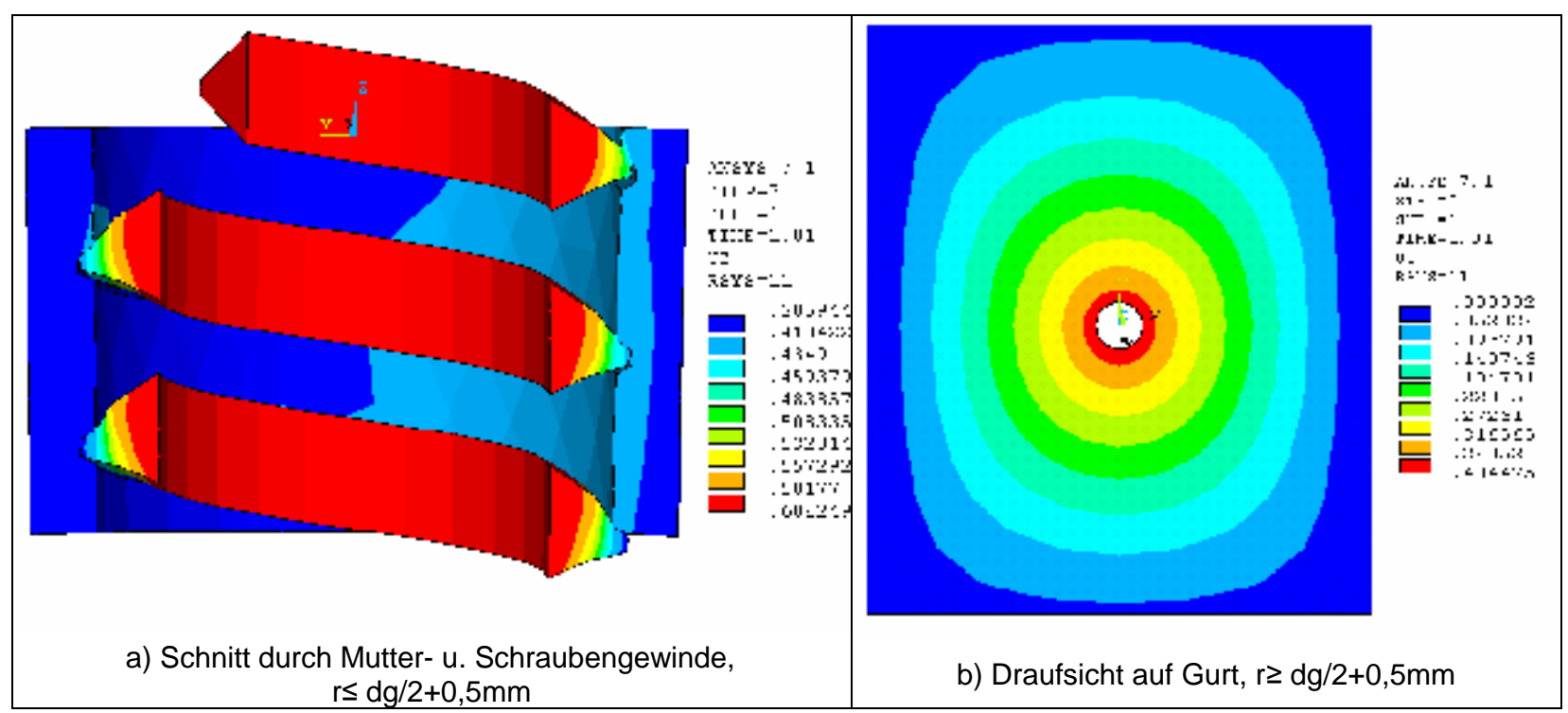

Bild 4.11: FE-Berechnung $A \_N R L \_400 S$, vertikale Verschiebung $u_{z}$ beim Erreichen der Maximallast 


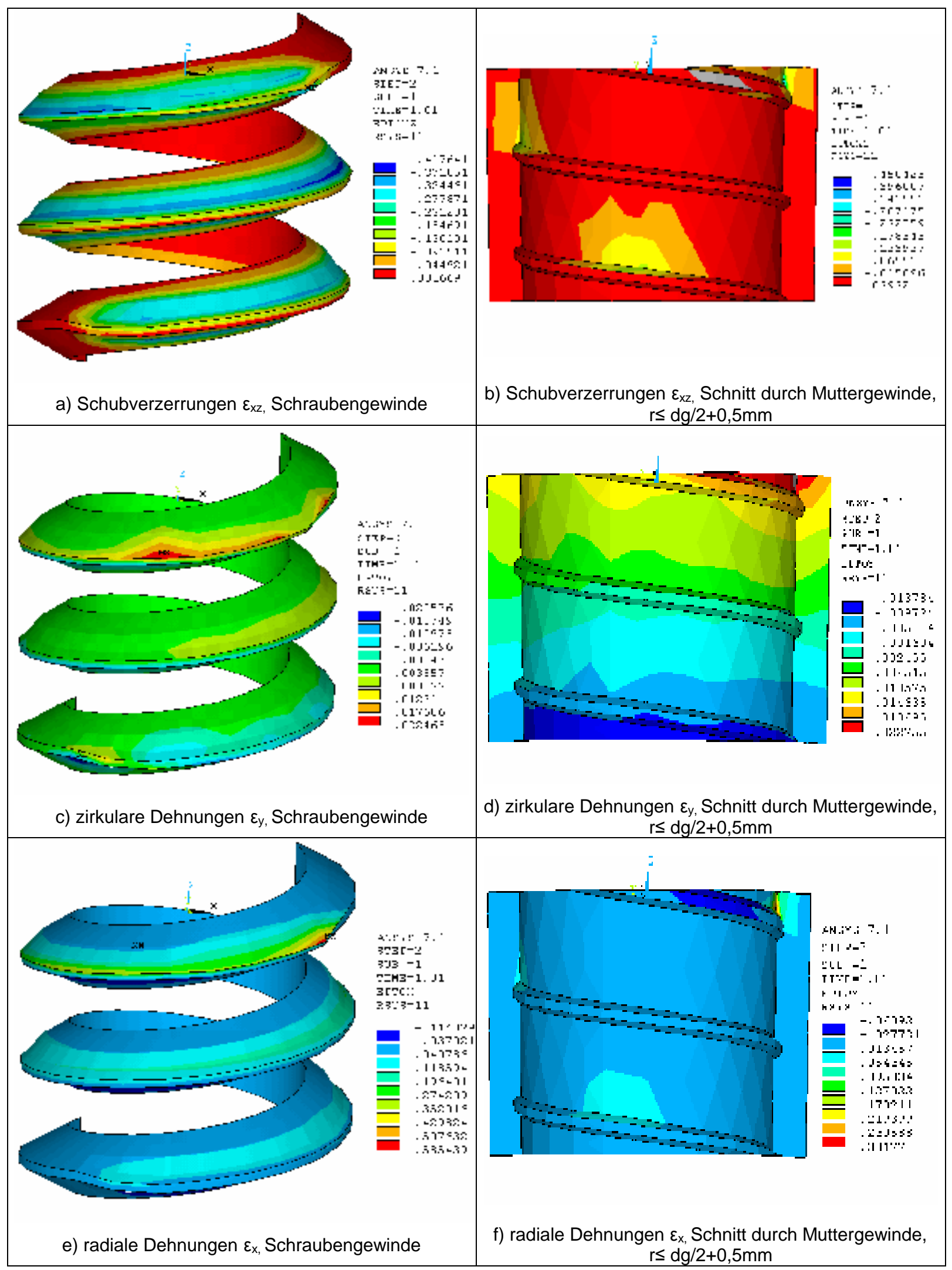

Bild 4.12: FE-Berechnung A_NRL_400S, Dehnungen $\varepsilon_{x z}, \varepsilon_{y}, \varepsilon_{x}$ in den Gewindegängen des Schrauben- und Muttergewindes beim Erreichen der Maximallast 

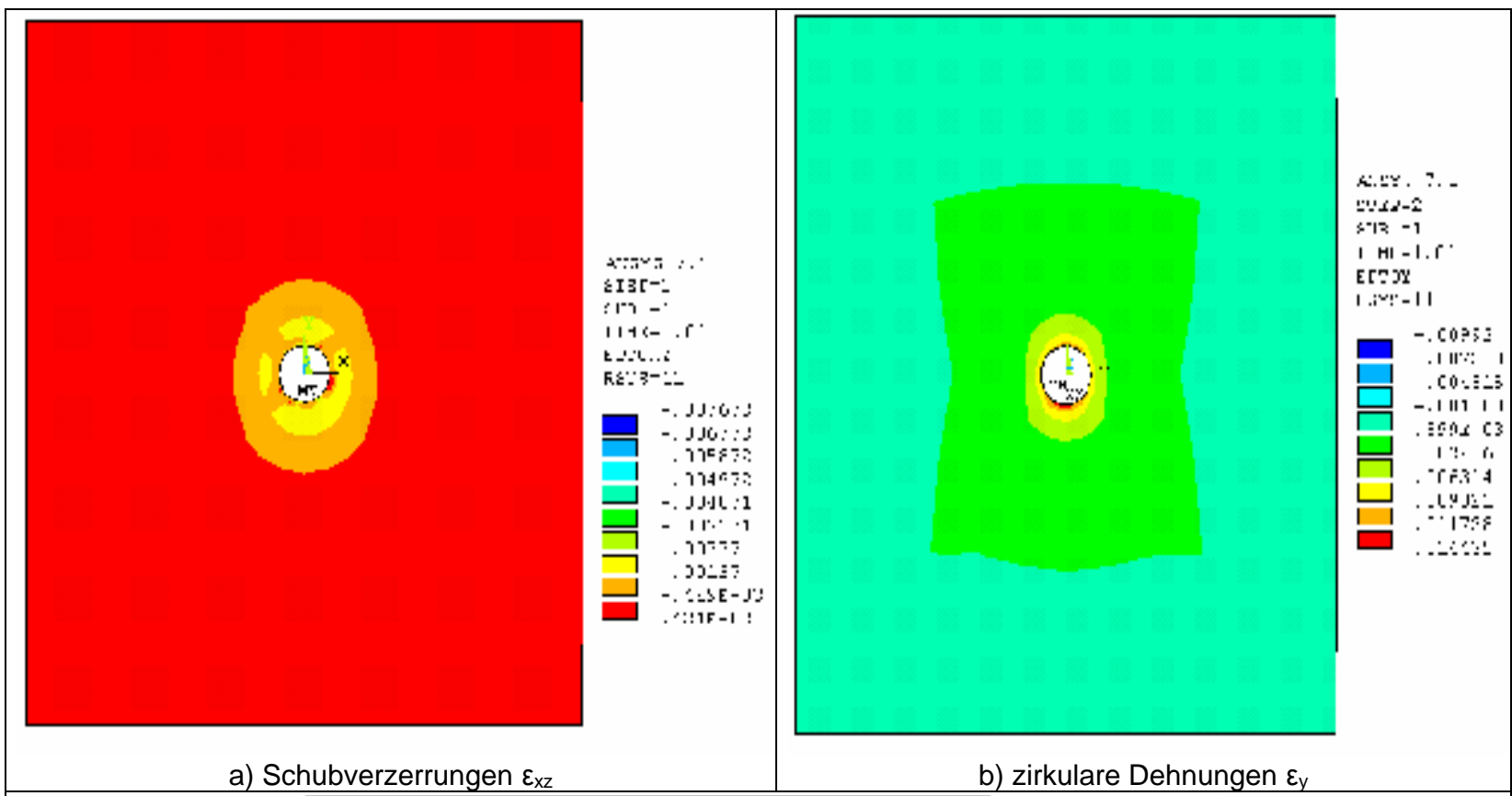

a) Schubverzerrungen $\varepsilon_{x z}$

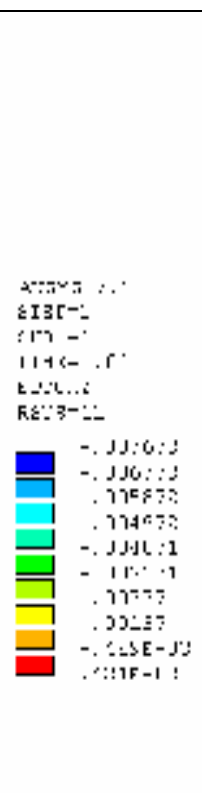

b) zirkulare Dehnungen $\varepsilon_{y}$

d) radiale Dehnungen $\varepsilon_{x}$

Bild 4.13: FE-Berechnung A_NRL_400S, Dehnungen $\varepsilon_{\mathrm{xz}}, \varepsilon_{\mathrm{y}}, \varepsilon_{\mathrm{x}}$ in der Unterkonstruktion auBerhalb des Muttergewindes ( $r \geq \mathrm{dg} / 2+0,5 \mathrm{~mm}$ ) beim Erreichen der Maximallast, Draufsicht auf Gurt 


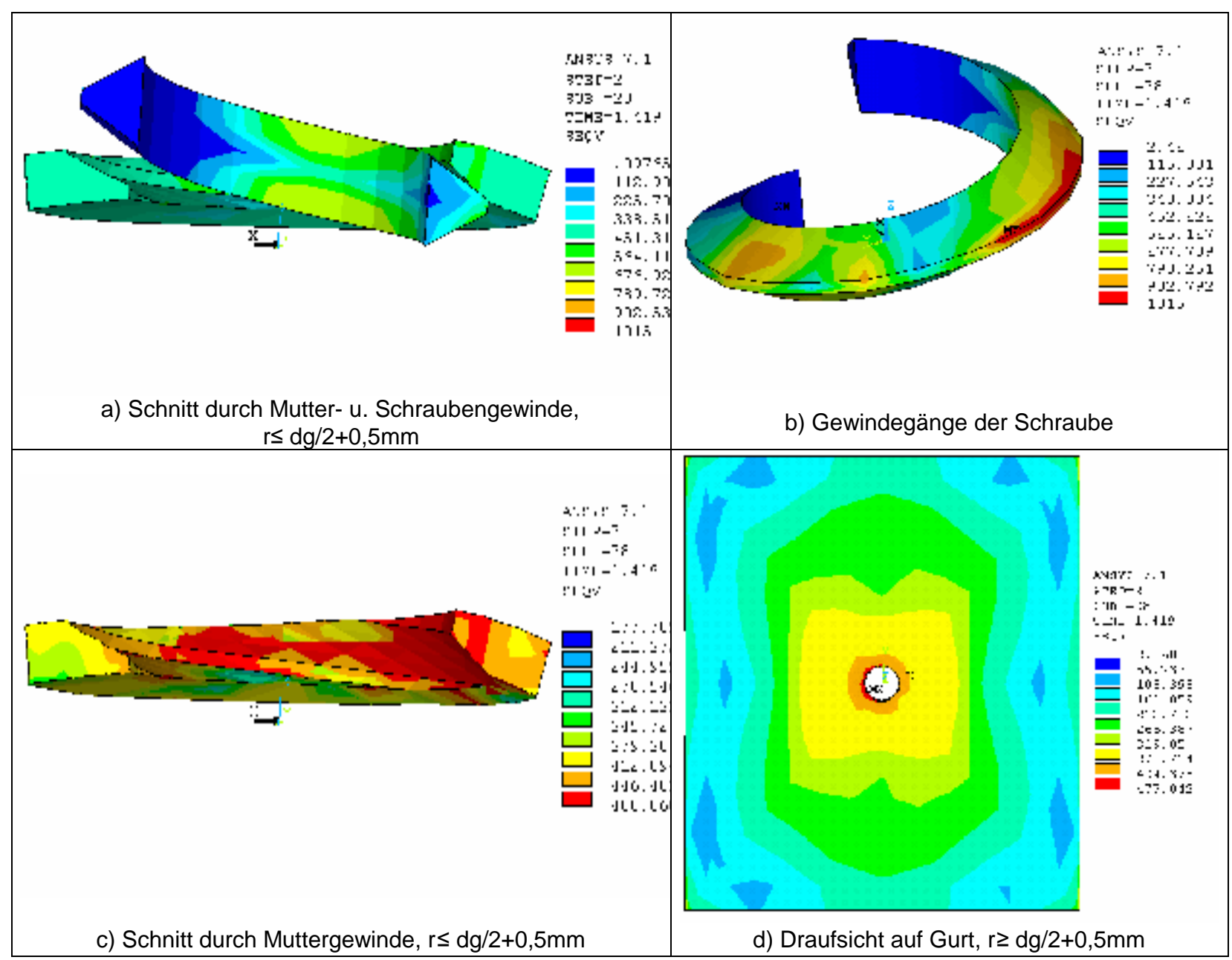

Bild 4.14: FE-Berechnung D_NRH_63S, Vergleichsspannung beim Erreichen der Maximallast

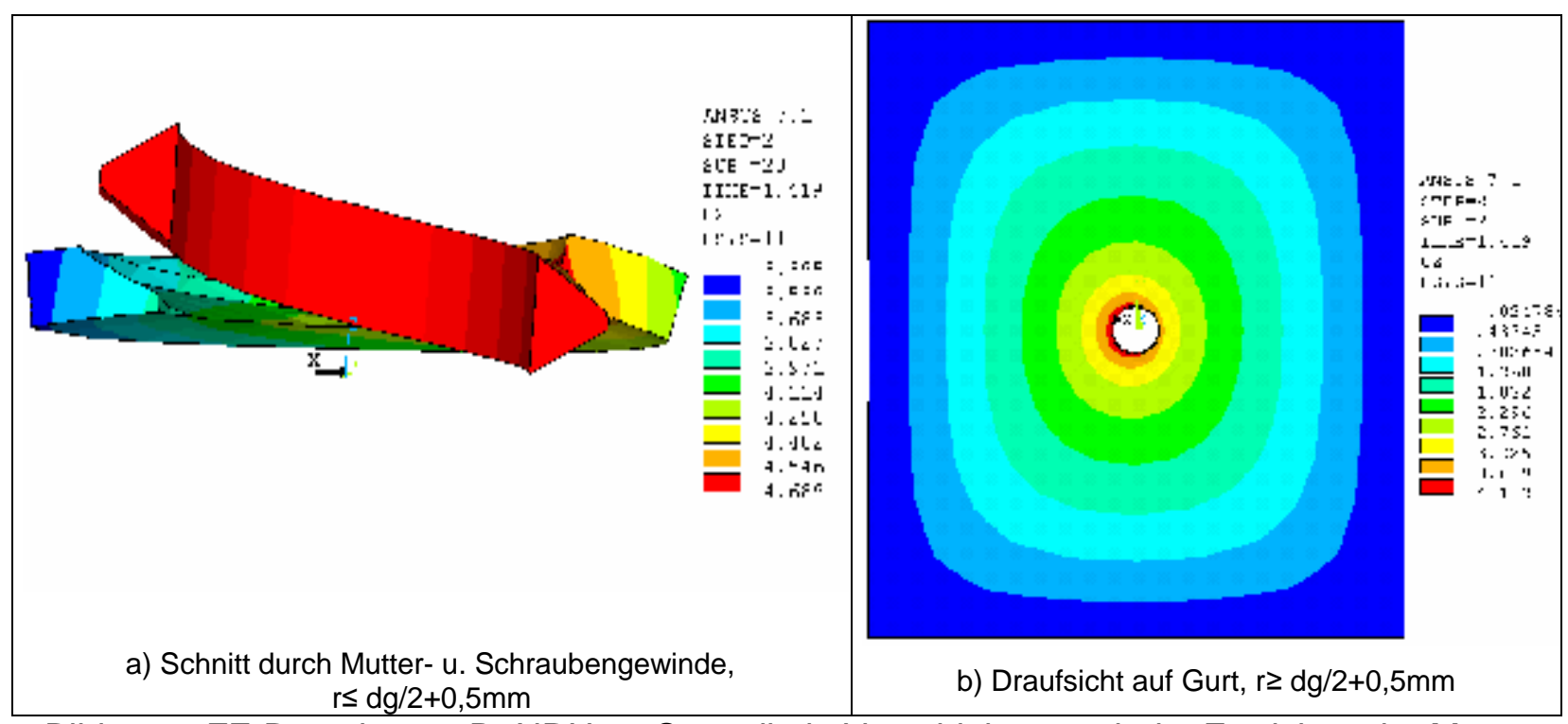

Bild 4.15: FE-Berechnung D_NRH_63S, vertikale Verschiebung uz beim Erreichen der Maximallast 


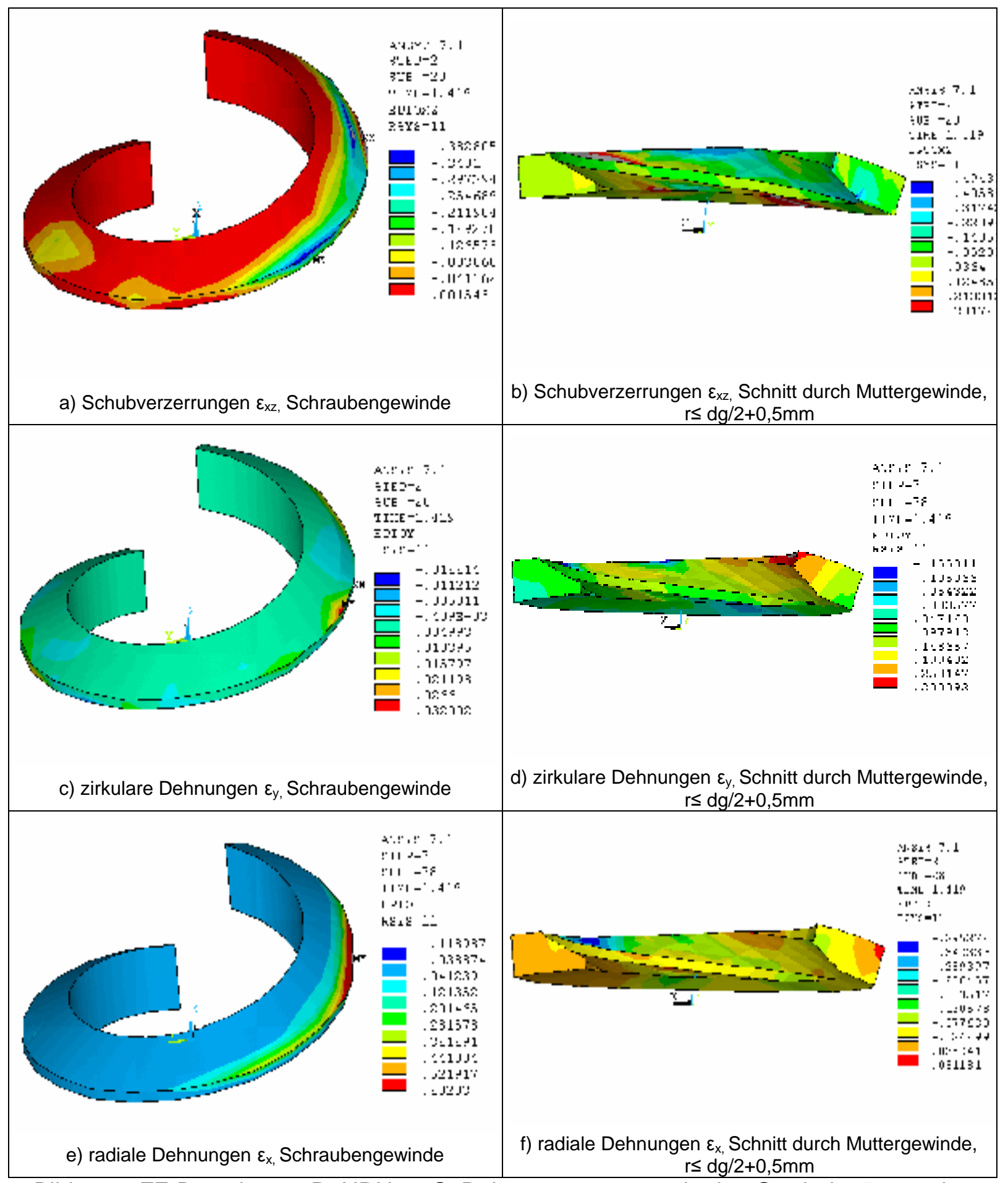

Bild 4.16: FE-Berechnung D_NRH_63S, Dehnungen $\varepsilon_{x z}, \varepsilon_{y}, \varepsilon_{x}$ in den Gewindegängen des Schrauben- und Muttergewindes beim Erreichen der Maximallast 


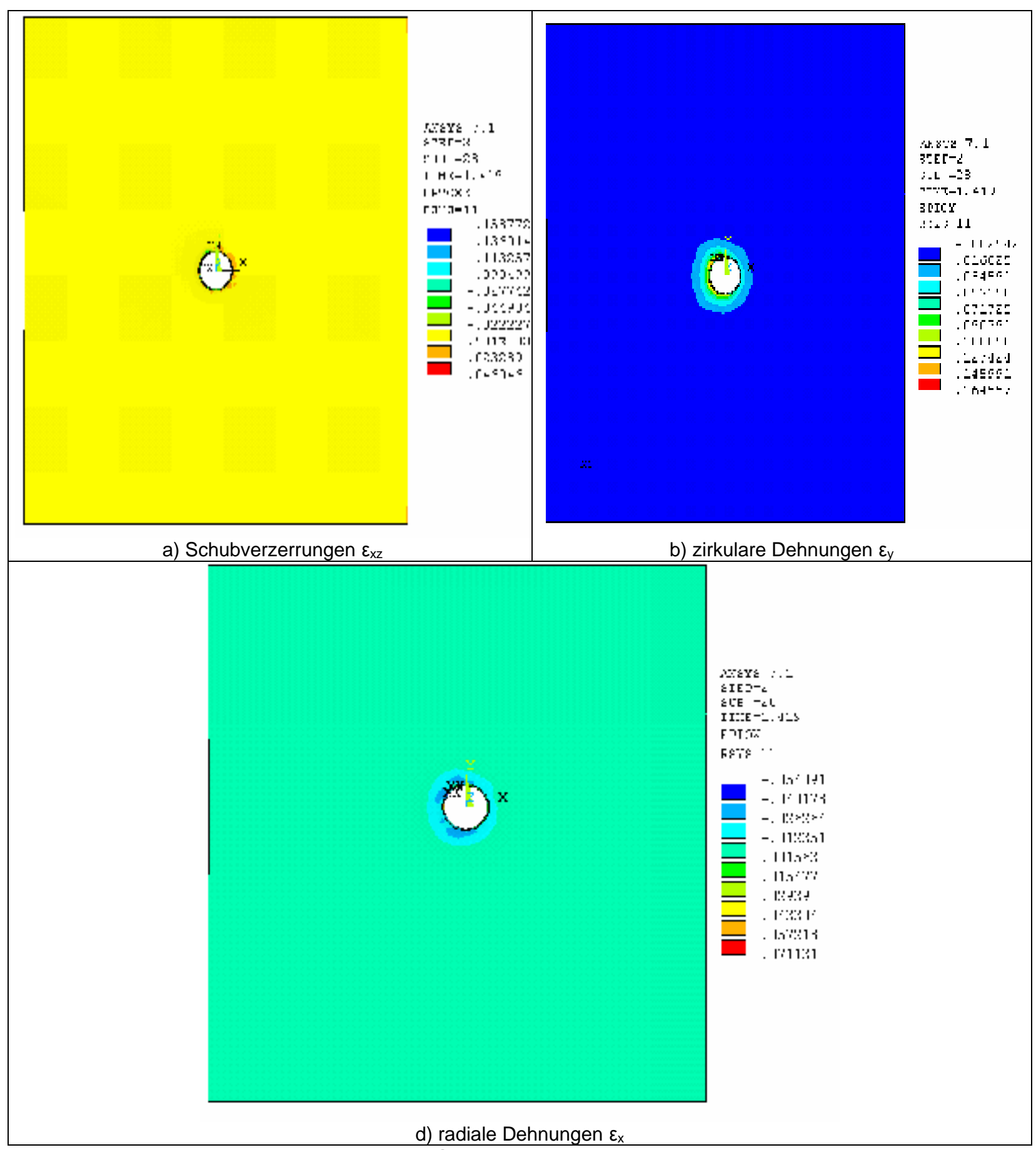

Bild 4.17: FE-Berechnung D_NRH_63S, Dehnungen $\varepsilon_{\mathrm{xz}}, \varepsilon_{\mathrm{y}}, \varepsilon_{\mathrm{x}}$ in der Unterkonstruktion auBerhalb des Muttergewindes ( $r \geq \mathrm{dg} / 2+0,5 \mathrm{~mm})$ beim Erreichen der Maximallast, Draufsicht auf Gurt 


\subsubsection{Vergleich der FE-Ergebnisse mit den Versuchsergebnissen}

In den Tabellen 4.5 bis 4.8 werden die in den FE-Berechnungen erreichten Maximallasten den in den jeweiligen Versuchsserien erreichten Versagenslasten gegenübergestellt.

In den Tabellen 4.5 bis 4.7 werden die FE-Berechnungen für die nichtrostenden Schrauben mit den jeweiligen Versuchsserien verglichen. In der Tabelle 4.5 sind die FE-Berechnungen aufgeführt, in denen das Werkstoffverhalten der Schrauben durch das Werkstoffgesetz „NRH“ (Anhang B, Diagramm B.15) und das Werkstoffverhalten der Unterkonstruktionen durch die aus den einaxialen Zugversuchen abgeleiteten Spannungs-Dehnungsbeziehungen (Anhang B, Diagramme B.1 bis B.11) abgebildet wurde. In der Tabelle 4.6 sind die FEBerechnungen aufgeführt, in denen für die Schrauben das Werkstoffgesetz „NRL“ (Anhang B, Diagramm B.16) und für die Unterkonstruktionen ebenfalls die aus den einaxialen Zugversuchen abgeleiteten Spannungs-Dehnungsbeziehungen verwendet wurden. Die Tabelle 4.7 enthält die Ergebnisse der FE-Berechnungen, in denen für die Schrauben das Werkstoffgesetz „NRH“ und für die Elemente im Bereich I der Unterkonstruktionen die Werkstoffgesetze mit den erhöhten Festigkeiten (Anhang B, Diagramme B.13 und B.14) verwendet wurden.

In der Tabelle 4.8 sind die FE-Berechnungen für die aus einem niedriglegierten Kohlenstoffstahl hergestellten Schraube „J“ den entsprechenden Versuchsergebnissen gegenübergestellt.

Aus dem Vergleich der FE-Ergebnisse mit den Versuchsergebnissen werden folgende Schlußfolgerungen gezogen:

- Aus den FE-Berechnungen für die nichtrostenden Schrauben, in denen für die Schrauben das Werkstoffgesetz „NRH“ und für die Unterkonstruktionen die aus den einaxialen Zugversuchen abgeleitetet Werkstoffgesetze verwendet wurden, ergeben sich Maximallasten, die gut mit den in den Versuchen erreichten mittleren Versagenslasten übereinstimmen (vgl. Tabelle 4.5). In den 28 FE-Berechnungen liegt der Betrag der Abweichungen zum Versuch in 17 Fällen unter 10\% und in 5 Fällen zwischen $10 \%$ und 15\%. In den verbleibenden 6 Fällen liegen die in den FE-Berechnungen erreichten Lasten maximal 21,4\% über (FE-Berechnung „C-NRH-150S“ für die Versuchsserie Nr. 191) und maximal 15,2\% unter (FE-Berechnung „A-NRH-400S“ für die Versuchsserie Nr. 207) der mittleren Versuchslast.

- Das Werkstoffgesetz „NRL“ wurde gemäß den Ausführungen im Abschnitt 4.5 als eine untere Abschätzung für die Werkstoffestigkeit von nichtrostenden Schrauben angesehen. Der Vergleich der FE-Berechnungen aus Tabelle 4.5 mit denjenigen aus Tabelle 4.6 zeigt, daß sich die gegenüber dem Werkstoffgesetz „NRH“ geringere Zugfestigkeit des Werkstoffgesetzes „NRL“ bei größer werdender Blechdicke und Zugfestigkeit der Unterkonstruktion zunehmend ungünstiger auf die erreichten Maximallasten auswirkt. Die 
größte Abweichung zwischen den FE-Berechnungen mit den unterschiedlichen Werkstoffgesetzen tritt in der Versuchsserie Nr. 207 für die Schraube „A“ und der Stahlunterkonstruktion mit der Nennblechdicke 4,00mm auf. Das Verhältnis der in diesen Berechnungen erreichten Versagenslasten $F_{\mathrm{FE}, \mathrm{NRL}} / \mathrm{F}_{\mathrm{FE}, \mathrm{NRH}}=0,85$ entspricht dem Verhältnis der wahren Zugfestigkeiten der beiden Werkstoffgesetze $R_{m, w a h r, N R L} / R_{m, w a h r, N R H}=863 / 1016=0,85$. Dieser Sachverhalt, daß offenbar das Versagen für die Versuchsserie 207 durch die Werkstoffestigkeit des Schraubengewindes bestimmt wird, sowie die Tatsache, daß es sich bei der Schraube „A“ um diejenige nichtrostende Schraube handelt, die gemäß der Tabelle 4.2, Abschnitt 4.2 die größte Zugfestigkeit aller dort aufgeführten nichtrostenden Schrauben aufweist, erklärt die festgestellte Abweichung von -15,2\% zwischen der in der FE-Berechnung „A-NRH-400S“ erreichten Maximallast und der mittleren Versagenslast der Versuchsserie 207. Offensichtlich wird selbst noch durch das Werkstoffgesetz „NRH“ die Werkstoffestigkeit der Schraube „A“ unterschätzt.

- Der Vergleich der FE-Berechnungen aus Tabelle 4.5 mit denjenigen aus Tabelle 4.7 zeigt, daß sich die im Bereich I des FE-Modells angenommene größere Werkstoffestigkeit der Unterkonstruktion in den Berechnungen für die Nennblechdicke $t_{N}=0,63 \mathrm{~mm}$ deutlich tragfähigkeitssteigernd auswirkt (die in den Berechnungen „63S_v“ erreichten Maximallasten liegen maximal 20\% und minimal 14\% über den Maximallasten der Berechnungen „63S“), während in den FE-Berechnungen für die Unterkonstruktion mit der Nennblechdicke $t_{N}=4,00 \mathrm{~mm}$ kein signifikanter Unterschied in den erreichten Maximallasten festzustellen ist (die Abweichungen zwischen den FE-Berechnungen „400S“ und „400S_v“ betragen ca. 1\%). Dies verdeutlicht nochmals den Sachverhalt, daß die Auszugstragfähigkeit für nichtrostende Schrauben bei große Dicken und Zugfestigkeiten der Unterkonstruktionen im wesentlichen von den geometrischen- und werkstoffspezifischen Eigenschaften der Schrauben abhängig ist, während die Auszugstragfähigkeit bei kleinen Unterkonstruktionsdicken zusätzlich zur Schraubengeometrie im wesentlichen durch das Werkstoffverhalten der Unterkonstruktionen bestimmt wird. Da die durch den Gewindeformvorgang erzielte Kaltverfestigung im Muttergewinde vor allem auf die oberflächennahen Randbereiche beschränkt bleibt (vgl. Kapitel 4.5), und da die in den FEBerechnungen „63S_v“ erreichten Maximallasten alle über den maximalen Versagenslasten der jeweiligen Versuchsserien liegen, wird die 20\%-ige Erhöhung der Werkstoffestigkeit der Elemente im gesamten Bereich I der Unterkonstruktion als eine Überschätzung des Effektes der Kaltverfestigung angesehen.

- Die Variation der Gewindeabflachung c von einem Wert c=0,15 für die Schraube "C“ auf einen Wert von 0,10 für die Schraube „Cvc“ macht sich in den erreichten Maximallasten der FE-Berechnungen mit maximal 6\% bemerkbar, wobei für die Schraube „Cvc“ stets 
kleinere Maximallasten erzielt werden (vgl. Tabellen 4.5 bis 4.7). Auch wenn dieser Einfluß der Gewindeabflachung $c$ auf die erreichte Maximallast als eher gering zu bezeichnen ist, verdeutlicht er doch, daß die festgestellten Abweichungen zwischen den FEBerechnungen und den Versuchsergebnissen auch auf Abweichungen zwischen den in den FE-Berechnungen angenommenen Maßangaben für das Schraubengewinde und den in den Versuchen tatsächlich vorliegenden Gegebenheiten zurückzuführen sind.

- Die in den FE-Berechnungen mit der aus einem niedriglegierten Kohlenstoffstahl hergestellten Schraube „J“ erreichten Maximallasten passen gut zu den in den Versuchen erreichten Versagenslasten (vgl. Tabelle 4.8). Die Maximallasten der FE-Berechnungen liegen alle innerhalb des Streubereiches der Versuchslasten. Während sich der Übergang von dem zur Beschreibung des Werkstoffverhaltens der Schraube verwendeten Werkstoffgesetz "CL“ auf das Werkstoffgesetz mit der erhöhten Zugfestigkeit „CH“ noch mit einer maximal um ca. 5\% größeren Maximallast auswirkt, bleiben die Abweichungen zwischen den FE-Berechnungen mit den für die Schraube verwendeten Werkstoffgesetzten "CH“ und "CH_CHR“ unter 1\%. Dies verdeutlicht den Sachverhalt, daß die in der FEBerechnung berücksichtigte erhöhte Festigkeit der oberflächennahen Bereiche der Gewindegänge der Schraube nahezu keinen Einfluß auf die Auszugstragfähigkeit besitzt. Die Variation des Gewindereibungskoeffizienten $\mu_{G}$ von einem Wert $\mu_{G}=0,35$ auf einen Wert $\mu_{G}=0,10$ macht sich in den erreichten Maximallasten der FE-Berechnungen nur maximal mit 3\% bemerkbar, so daß der Einfluß der Gewindereibung auf die Auszugstragfähigkeit von gewindeformenden Schrauben als vernachlässigbar anzusehen ist. 
Tabelle 4.5: Vergleich zwischen den Versuchen mit nichtrostenden Schrauben und den FEBerechnungen, in denen für die Schrauben das Werkstoffgesetz „NRH“ und für die Unterkonstruktionen die aus den einaxialen Zugversuchen abgeleiteten Werkstoffgesetzte verwendet wurden

\begin{tabular}{|c|c|c|c|c|c|c|}
\hline \multirow{3}{*}{\begin{tabular}{|} 
Lfd. Nr. \\
vgl. \\
Abs. 4.2, \\
Tab. 4.1
\end{tabular}} & \multicolumn{3}{|c|}{ Versuch } & \multicolumn{2}{|c|}{$\begin{array}{c}\text { FE-Berechnung } \\
\text { Gewindereibungskoeffiz:: } \mu_{g}=0,35\end{array}$} & \multirow{3}{*}{$\begin{array}{c}\text { Abwei- } \\
\text { chung } \\
\left(\begin{array}{c}F_{\mathrm{FE}}-F_{\mathrm{V}, \mathrm{m}} \\
F_{\mathrm{V}, \mathrm{m}} \\
{ }^{*} 100 \%\end{array}\right.\end{array}$} \\
\hline & \multicolumn{3}{|c|}{ Versagenslast [kN] } & \multirow{2}{*}{$\begin{array}{l}\text { FE- } \\
\text { Bezeich- } \\
\text { nung }^{1)}\end{array}$} & \multirow{2}{*}{$\begin{array}{c}\text { Max. Last } \\
\text { F }_{\mathrm{FE}}[\mathrm{kN}]\end{array}$} & \\
\hline & $\begin{array}{c}\text { Mittel } \\
\mathrm{F}_{\mathrm{v}, \mathrm{m}}\end{array}$ & Max. & Min. & & & \\
\hline 207 & 9,829 & 10,178 & 9,091 & A-NRH-400S & 8,335 & $-15,2$ \\
\hline 175 & 1,186 & 1,290 & 1,060 & B-NRH-63S & 1,143 & $-3,6$ \\
\hline 176 & 2,420 & 2,580 & 2,240 & B-NRH-75S & 2,450 & 1,3 \\
\hline 177 & 3,800 & 3,990 & 3,640 & B-NRH-150S & 3,542 & $-6,8$ \\
\hline \multirow{2}{*}{191} & \multirow{2}{*}{2,078} & \multirow{2}{*}{2,266} & \multirow{2}{*}{1,886} & C-NRH-150S & 2,522 & 21,4 \\
\hline & & & & Cvc-NRH-150S & 2,466 & 18,7 \\
\hline \multirow{2}{*}{192} & \multirow{2}{*}{3,051} & \multirow{2}{*}{3,580} & \multirow{2}{*}{2,690} & C-NRH-200S & 3,454 & 13,2 \\
\hline & & & & Cvc-NRH-200S & 3,354 & 9,9 \\
\hline \multirow{2}{*}{193} & \multirow{2}{*}{5,114} & \multirow{2}{*}{5,330} & \multirow{2}{*}{4,810} & C-NRH-300S & 5,124 & 0,2 \\
\hline & & & & Cvc-NRH-300S & 5,096 & $-0,4$ \\
\hline \multirow{2}{*}{194} & \multirow{2}{*}{9,684} & \multirow{2}{*}{10,276} & \multirow{2}{*}{9,250} & C-NRH-400S & 10,546 & 8,9 \\
\hline & & & & Cvc-NRH-400S & 9,990 & 3,2 \\
\hline 172 & 0,943 & 1,090 & 0,843 & D-NRH-63S & 1,089 & 15,5 \\
\hline 173 & 1,815 & 1,920 & 1,540 & D-NRH-100S & 2,073 & 14,2 \\
\hline 174 & 3,021 & 3,220 & 2,830 & D-NRH-150S & 3,158 & 4,5 \\
\hline 182 & 1,030 & 1,100 & 0,940 & E-NRH-75S & 1,017 & $-1,3$ \\
\hline 183 & 1,720 & 1,800 & 1,610 & E-NRH-100S & 1,910 & 11,0 \\
\hline 185 & 2,864 & 3,110 & 2,750 & E-NRH-150S & 2,876 & 0,4 \\
\hline 186 & 4,028 & 4,250 & 3,870 & E-NRH-200S & 3,929 & $-2,4$ \\
\hline 141 & 1,184 & 1,211 & 1,134 & F1-NRH-63S & 1,035 & $-12,6$ \\
\hline 142 & 1,290 & 1,328 & 1,172 & F1-NRH-75S & 1,175 & $-8,9$ \\
\hline 143 & 2,097 & 2,210 & 1,950 & F1-NRH-100S & 2,215 & 5,6 \\
\hline 145 & 2,907 & 3,040 & 2,660 & F2-NRH-150S & 3,128 & 7,6 \\
\hline 181 & 13,307 & 14,083 & 12,229 & G-NRH-500S & 12,873 & $-3,3$ \\
\hline 229 & 1,524 & 1,673 & 1,458 & $\mathrm{H}-\mathrm{NRH}-150 \mathrm{~A}$ & 1,545 & 1,4 \\
\hline 230 & 2,419 & 2,580 & 2,310 & H-NRH-200A & 2,705 & 11,8 \\
\hline 227 & 2,080 & 2,128 & 2,050 & I-NRH-200A & 2,468 & 18,7 \\
\hline 228 & 3,616 & 3,795 & 3,487 & I-NRH-300A & 4,244 & 17,4 \\
\hline \multicolumn{5}{|c|}{ 1) FE-Bezeichnung: Bsp. A-NRH-400S } & Max. & 21,4 \\
\hline $\begin{array}{ll}\text { A: } & \text { Sc } \\
\text { NRH: } & \text { Sc } \\
\text { 400S: } & \text { Ur } \\
& \text { vg }\end{array}$ & \multicolumn{4}{|c|}{$\begin{array}{l}\text { Schraube: vgl. Abs. } 4.2 \text {, Tabelle } 4.2 \\
\text { Schraubenwerkstoff: vgl. Anhang B, Diagr. B.15 } \\
\text { Unterkonstruktion: vgl. Abs. 4.2, Tabelle 4.3, Werkstoffgesetz } \\
\text { vgl. Anhang B, Diagr. B.1-B.11 }\end{array}$} & Min.: & $-15,2$ \\
\hline
\end{tabular}


Tabelle 4.6: Vergleich zwischen den Versuchen mit nichtrostenden Schrauben und den FEBerechnungen, in denen für die Schrauben das Werkstoffgesetz „NRL “ und für die Unterkonstruktionen die aus den einaxialen Zugversuchen abgeleiteten Werkstoffgesetzte verwendet wurden

\begin{tabular}{|c|c|c|c|c|c|c|}
\hline \multirow{3}{*}{\begin{tabular}{|} 
Lfd. Nr. \\
vgl. \\
Abs. 4.2, \\
Tab. 4.1
\end{tabular}} & \multicolumn{3}{|c|}{ Versuch } & \multicolumn{2}{|c|}{$\begin{array}{c}\text { FE-Berechnung } \\
\text { Gewindereibungskoeffiz:: } \mu_{g}=0,35\end{array}$} & \multirow{3}{*}{$\begin{array}{c}\text { Abwei- } \\
\text { chung } \\
\left(\begin{array}{c}F_{\mathrm{FE}}-F_{\mathrm{V}, \mathrm{m}} \\
F_{\mathrm{V}, \mathrm{m}} \\
{ }^{*} 100 \%\end{array}\right.\end{array}$} \\
\hline & \multicolumn{3}{|c|}{ Versagenslast [kN] } & \multirow{2}{*}{$\begin{array}{l}\text { FE- } \\
\text { Bezeich- } \\
\text { nung }^{1)}\end{array}$} & \multirow{2}{*}{$\begin{array}{c}\text { Max. Last } \\
\text { F }_{\mathrm{FE}}[\mathrm{kN}]\end{array}$} & \\
\hline & $\begin{array}{c}\text { Mittel } \\
\mathrm{F}_{\mathrm{v}, \mathrm{m}}\end{array}$ & Max. & Min. & & & \\
\hline 207 & 9,829 & 10,178 & 9,091 & A-NRL-400S & 7,076 & $-28,0$ \\
\hline 175 & 1,186 & 1,290 & 1,060 & B-NRL-63S & 1,109 & $-6,5$ \\
\hline 176 & 2,420 & 2,580 & 2,240 & B- NRL -75S & 2,381 & $-1,6$ \\
\hline 177 & 3,800 & 3,990 & 3,640 & B- NRL -150S & 3,449 & $-9,2$ \\
\hline \multirow{2}{*}{191} & \multirow{2}{*}{2,078} & \multirow{2}{*}{2,266} & \multirow{2}{*}{1,886} & C- NRL -150S & 2,412 & 16,1 \\
\hline & & & & Cvc-NRL -150S & 2,350 & 13,1 \\
\hline \multirow{2}{*}{192} & \multirow{2}{*}{3,051} & \multirow{2}{*}{3,580} & \multirow{2}{*}{2,690} & C-NRL-200S & 3,221 & 5,6 \\
\hline & & & & Cvc-NRL-200S & 3,136 & 2,8 \\
\hline \multirow{2}{*}{193} & \multirow{2}{*}{5,114} & \multirow{2}{*}{5,330} & \multirow{2}{*}{4,810} & C-NRL-300S & 4,910 & $-4,0$ \\
\hline & & & & Cvc-NRL-300S & 4,796 & $-6,2$ \\
\hline \multirow{2}{*}{194} & \multirow{2}{*}{9,684} & \multirow{2}{*}{10,276} & \multirow{2}{*}{9,250} & C-NRL-400S & 9,320 & $-3,8$ \\
\hline & & & & Cvc-NRH-400S & 8,770 & $-9,4$ \\
\hline 172 & 0,943 & 1,090 & 0,843 & D-NRL-63S & 1,061 & 12,5 \\
\hline 173 & 1,815 & 1,920 & 1,540 & D-NRL-100S & 2,019 & 11,3 \\
\hline 174 & 3,021 & 3,220 & 2,830 & D-NRL-150S & 3,074 & 1,8 \\
\hline 182 & 1,030 & 1,100 & 0,940 & E-NRL-75S & 0,994 & $-3,5$ \\
\hline 183 & 1,720 & 1,800 & 1,610 & E-NRL-100S & 1,841 & 7,0 \\
\hline 185 & 2,864 & 3,110 & 2,750 & E-NRL-150S & 2,784 & $-2,8$ \\
\hline 186 & 4,028 & 4,250 & 3,870 & E-NRL-200S & 3,712 & $-7,8$ \\
\hline 141 & 1,184 & 1,211 & 1,134 & F1-NRL-63S & 1,014 & $-14,4$ \\
\hline 142 & 1,290 & 1,328 & 1,172 & F1-NRL-75S & 1,163 & $-9,8$ \\
\hline 143 & 2,097 & 2,210 & 1,950 & F1-NRL-100S & 2,165 & 3,2 \\
\hline 145 & 2,907 & 3,040 & 2,660 & F2-NRL-150S & 3,010 & 3,5 \\
\hline 181 & 13,307 & 14,083 & 12,229 & G-NRL-500S & 12,117 & $-8,9$ \\
\hline 229 & 1,524 & 1,673 & 1,458 & H-NRL-150A & 1,533 & 0,6 \\
\hline 230 & 2,419 & 2,580 & 2,310 & H-NRL-200A & 2,638 & 9,1 \\
\hline 227 & 2,080 & 2,128 & 2,050 & I-NRL-200A & 2,368 & 13,8 \\
\hline 228 & 3,616 & 3,795 & 3,487 & I-NRL-300A & 4,071 & 12,6 \\
\hline \multicolumn{5}{|c|}{ 1) FE-Bezeichnung: Bsp. A-NRL-400S } & Max. & 16,1 \\
\hline $\begin{array}{ll}\text { A: } & \text { Sc } \\
\text { NRL: } & \text { Sc } \\
\text { 400S: } & \text { Ur } \\
& \text { vg }\end{array}$ & \multicolumn{4}{|c|}{$\begin{array}{l}\text { Schraube: vgl. Abs. 4.2, Tabelle } 4.2 \\
\text { Schraubenwerkstoff: vgl. Anhang B, Diagr. B.16 } \\
\text { Unterkonstruktion: vgl. Abs. 4.2, Tabelle 4.3, Werkstoffgesetz } \\
\text { vgl. Anhang B, Diagr. B.1-B.11 }\end{array}$} & Min.: & $-28,0$ \\
\hline
\end{tabular}


Tabelle 4.7: Vergleich zwischen den Versuchen mit nichtrostenden Schrauben und den FEBerechnungen, in denen für die Schrauben das Werkstoffgesetz „NRH“ und für die Elemente im Bereich I der Unterkonstruktionen die Werkstoffgesetze mit den erhöhten Festigkeiten verwendet wurden

\begin{tabular}{|c|c|c|c|c|c|c|}
\hline & Ver & uch & & $\begin{array}{r}\text { FE-Berecl } \\
\text { Gewindereibungsk }\end{array}$ & $\begin{array}{l}\mathrm{gg}: \mu_{g}=0,35 \\
\text { la }\end{array}$ & $\begin{array}{l}\text { Abwei- } \\
\text { chung }\end{array}$ \\
\hline Lfd. Nr. & Ver & agensla & {$[\mathrm{kN}]$} & FE- & & $\left(F_{\mathrm{FE}}-\mathbf{F}_{\mathrm{V}, \mathrm{m}}\right)$ \\
\hline $\begin{array}{l}\text { vgl. } \\
\text { Abs. } 4.2, \\
\text { Tab. } 4.1\end{array}$ & $\begin{array}{c}\text { Mittel } \\
\mathrm{F}_{\mathrm{v}, \mathrm{m}}\end{array}$ & Max. & Min. & $\begin{array}{l}\text { Bezeich- } \\
\text { nung }{ }^{1}\end{array}$ & $\begin{array}{l}\text { Miax. Last } \\
\mathrm{F}_{\mathrm{FE}}[\mathrm{kN}]\end{array}$ & $\begin{array}{c}\begin{array}{c}F_{V, m} \\
{ }^{*} 100 \%\end{array} \\
\end{array}$ \\
\hline 207 & 9,829 & 10,178 & 9,091 & A-NRH-400S_v & 8,406 & $-14,5$ \\
\hline 175 & 1,186 & 1,290 & 1,060 & B-NRH-63S_v & 1,307 & 10,2 \\
\hline & 0,01 & ת70 10 & & C-NRH-400S_v & 10,693 & 10,4 \\
\hline & & & & Cvc-NRH-400S_v & 10,134 & 4,6 \\
\hline 172 & 0,943 & 1,090 & 0,843 & D-NRH-63S_v & 1,244 & 31,9 \\
\hline 141 & 1,184 & 1,211 & 1,134 & F1-NRH-63S_v & 1,247 & 5,3 \\
\hline 1) FE-Beze & hnung & p. A- & $\mathrm{H}-4 \mathrm{C}$ & & Max. & 31,9 \\
\hline $\begin{array}{l}\text { A: } \\
\text { NRH: } \\
\text { 400S_v: }\end{array}$ & $\begin{array}{l}\text { Schrau } \\
\text { Schrau } \\
\text { Unterk } \\
\text { setz vgl }\end{array}$ & $\begin{array}{l}\text { : vgl. Ak } \\
\text { nwerks } \\
\text { struktic } \\
\text { hhang B }\end{array}$ & $\begin{array}{l}\text { 4.2, Ta } \\
\text { ff: vgl. } \\
\text { : vgl. A } \\
\text { iagr. B. }\end{array}$ & $\begin{array}{l}\text { le } 4.2 \\
\text { hang B, Diagr. B. } 15 \\
\text { 4.2, Tabelle } 4.3 \text {, Werks } \\
\text { und B.14 }\end{array}$ & Min.: & $-14,5$ \\
\hline
\end{tabular}

Tabelle 4.8: FE-Berechnungen für die Schraube „J“

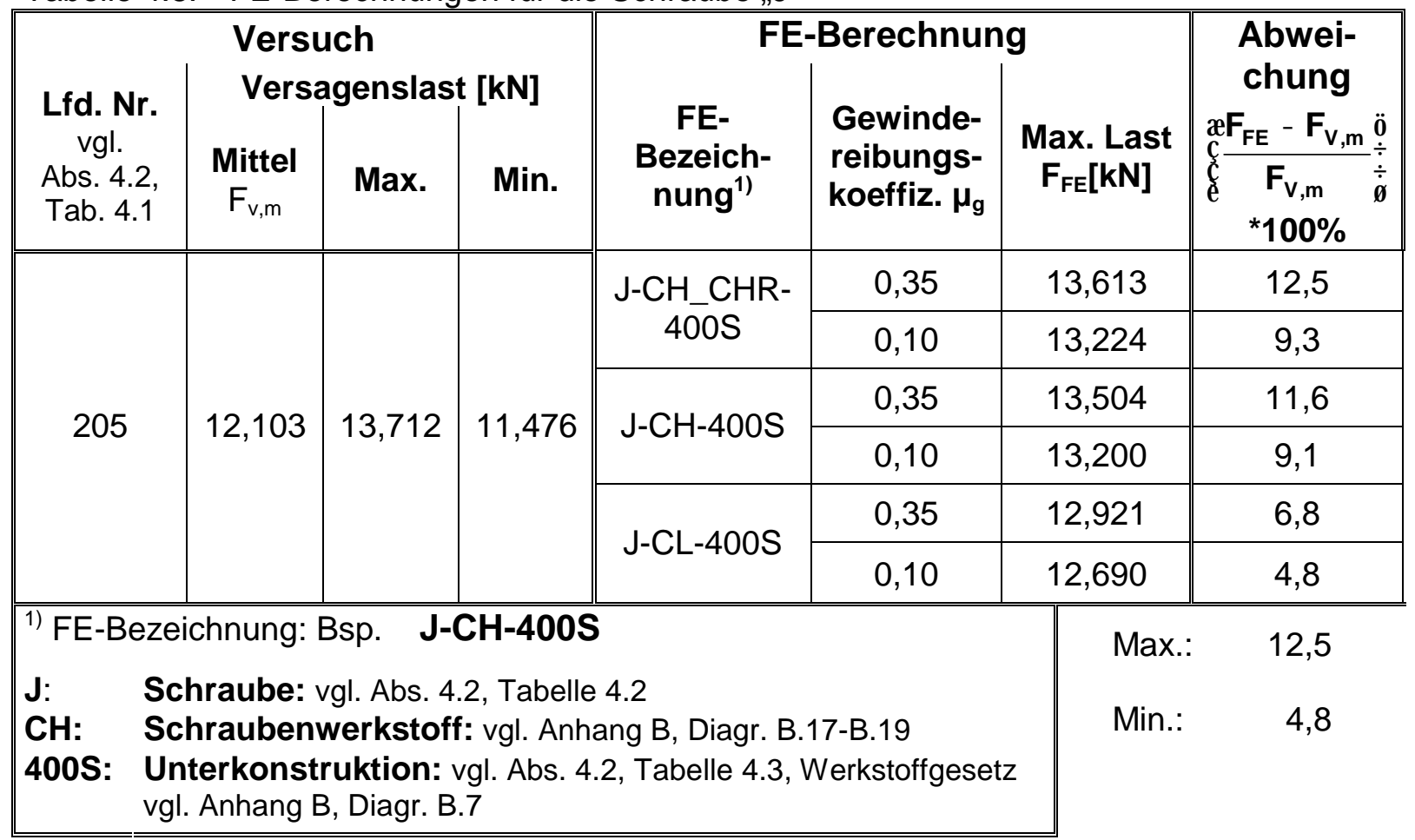


In den Diagrammen C.1 bis C.12 im Anhang C sind für die verschiedenen Schrauben die Last-Verformungsbeziehungen der FE-Berechnungen den Last-Verformungsbeziehungen aus den Versuchen gegenübergestellt.

Für die FE-Berechnungen wurde in den Diagrammen die Summe der Auflagerkräfte an den Querrändern der Unterkonstruktion über die vertikale Verschiebung der belasteten Schraubenknoten in Richtung der Schraubenachse aufgetragen.

Für die Versuche ist in den Diagrammen der Verlauf der Kraft über der Verschiebung des Querhauptes der Prüfmaschine - in diesem wird die Auflagervorrichtung für die Unterkonstruktion eingespannt (Bild 2.14, Abschnitt 2.3) - aufgetragen. Für jede Versuchsserie wurde sowohl die Last-Verformungsbeziehung des Versuchs mit der größten in der Versuchsserie erreichten Versagenslast (Kurve „Vers._Fmax“) als auch der Versuch mit der kleinsten in der Versuchsserie erreichten Versagenslast (Kurve „Vers._Fmin“) in den Diagrammen dargestellt.

Aus den Diagrammen wird ersichtlich, daß für Nennblechdicken der Unterkonstruktionen $t_{N} \leq 3,00 \mathrm{~mm}$ die Last-Verformungsbeziehungen der Versuche gut mit denen der FEBerechnungen übereinstimmen.

Für größere Nennblechdicken $\left(t_{N}=4,00 \mathrm{~mm}\right.$ und $\left.t_{N}=5,00 \mathrm{~mm}\right)$ liefern die FE-Berechnungen im Vergleich zu den Versuchen sehr viel größere Steifigkeiten (vgl. Anhang C, Diagramme C.1, C.3, C.4, C.8, C.11 und C.12).

Wie aus den Diagrammen C.3 und C.4 ersichtlich wird, wachsen die Anfangssteifigkeiten der Versuche mit größer werdenden Nennblechdicken bis $t_{N}=3,00 \mathrm{~mm}$ kontinuierlich an, während beim Übergang von $t_{N}=3,00 \mathrm{~mm}$ auf $t_{N}=4,00 \mathrm{~mm}$ kein Anstieg mehr in den Anfangssteifigkeiten zu verzeichnen ist. Im Gegensatz dazu zeigen die FE-Berechnungen einen sehr deutlichen Anstieg der Anfangssteifigkeit beim Übergang von $t_{N}=3,00 \mathrm{~mm}$ auf $t_{N}=4,00 \mathrm{~mm}$.

Dieser Effekt ist zu erklären über die im FE-Modell nicht abgebildete Nachgiebigkeit, die sich in den Versuchen durch die Auflagerung des Schraubenkopfes in der Einspannvorrichtung (Bild 2.14, Abschnitt 2.3) ergibt, sowie durch die im FE-Modell überschätzte Steifigkeit der im Versuch verwendeten Auflagervorrichtung für die Unterkonstruktion (Bild 2.14, Abschnitt 2.3). Dies wurde anhand von Versuchen verdeutlicht, deren Versuchsaufbau schematisch im Bild 4.18 dargestellt ist. 


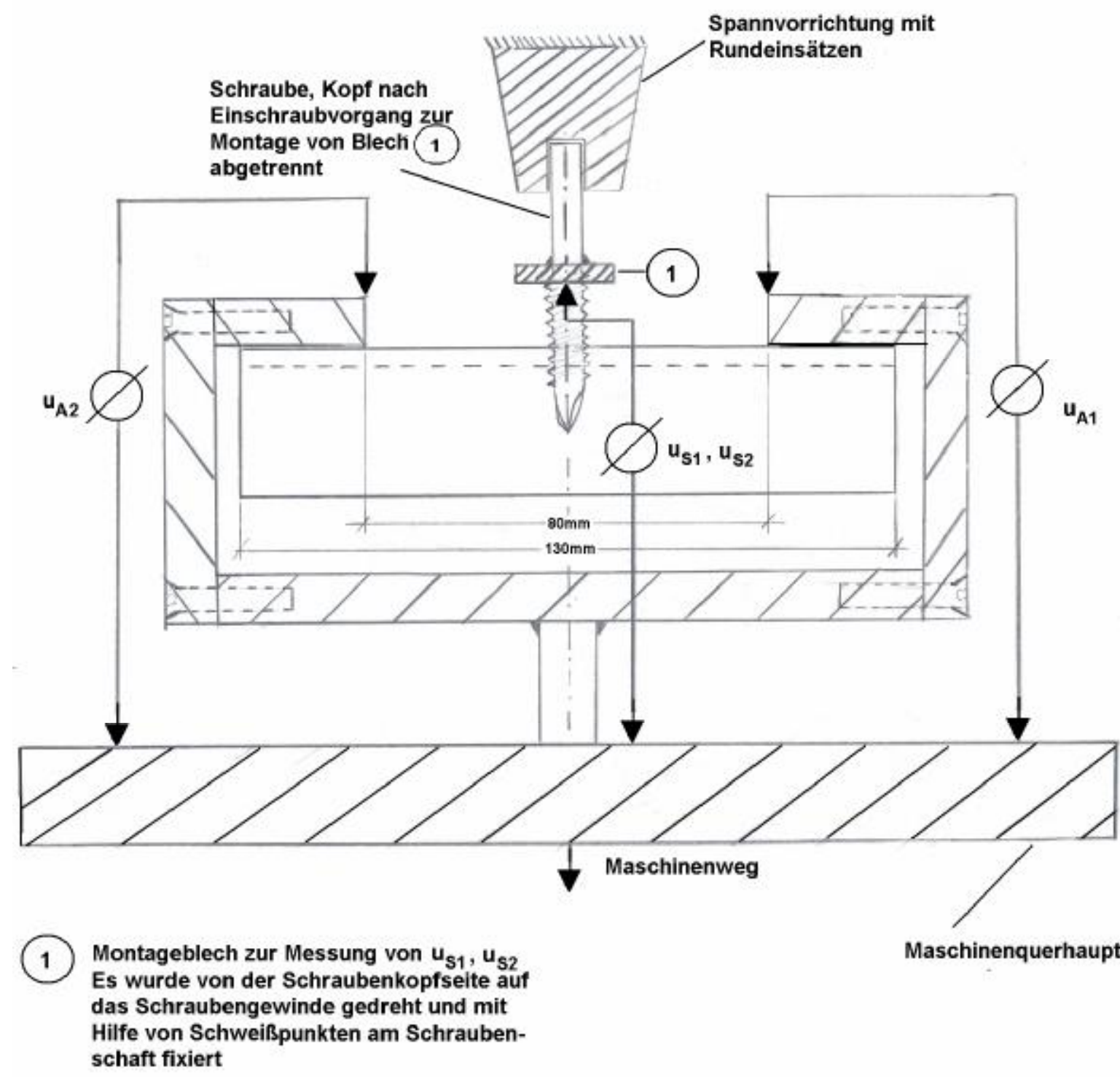

Bild 4.18: Versuchsaufbau zur Eliminierung der Schraubenkopfnachgiebigkeit und zur Bestimmung der Steifigkeit der Auflagervorrichtung für die Unterkonstruktion

Die Versuche wurden mit den Schrauben Nr. 61 (in den FE-Brechnungen mit „C“ und „Cvc“ bezeichnet) und Nr. 67 (in den FE-Brechnungen mit „A“ bezeichnet) durchgeführt. Für die Unterkonstruktion wurde derjenige Werkstoff verwendet, welcher in den FE-Berechnungen mit 400S bezeichnet wurde (Charge Nr. 100 in der Versuchsdatenbank, Anhang A, Tabelle A.1). Für jeden Schraubentyp wurden zwei Versuche durchgeführt. In jeweils einem Versuch pro Schraubentyp wurde die Verschiebung des Schraubenschaftes gegenüber dem Querhaupt der Prüfmaschine über zwei Wegaufnehmer $\left(u_{s 1}\right.$ und $u_{s 2}$, Bild 4.18) gemessen, so daß die in den FE-Berechnungen nicht modellierten Nachgiebigkeiten an der Einspannung des Schraubenkopfes eliminiert wurden. In dem jeweils zweiten Versuch für jede Schraube wurden mit Hilfe zweier weiterer Wegaufnehmer die Relativverschiebungen $u_{A 1}$ und $u_{A 2} z w i-$ schen der Auflagervorrichtung und dem Maschinenquerhaupt gemessen (Bild 4.18). Mit Hilfe dieser Messungen kann ein Vergleich angestellt werden zwischen der in den FE- 
Berechnungen angenommenen Steifigkeit für die Auflagervorrichtung der Unterkonstruktion und ihrer tatsächlich vorhandenen Steifigkeit. Die Versuchsergebnisse sind für die beiden Schrauben in den Diagrammen 4.7 und 4.8 den jeweiligen in der Tabelle 4.5 angegebenen FE-Berechnungen („A-NRH-400S“, „C-NRH-400S“ und „Cvc-NRH-400S“) sowie den ursprünglichen Versuchen aus Anhang C („Vers._Fmax“, „Vers._Fmin“) gegenübergestellt.

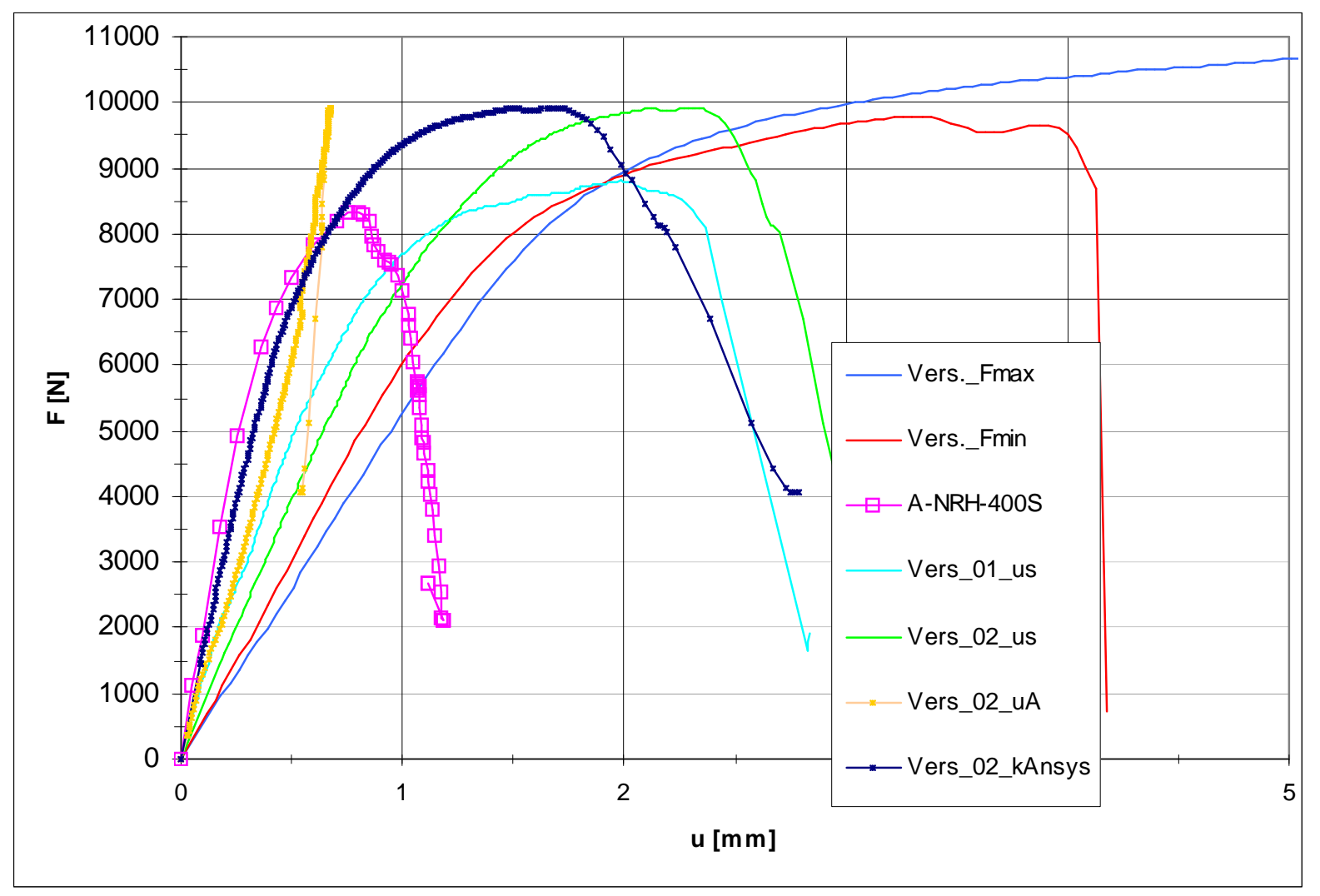

Diagramm 4.7: Versuche mit der Schraube Nr. 67=A 


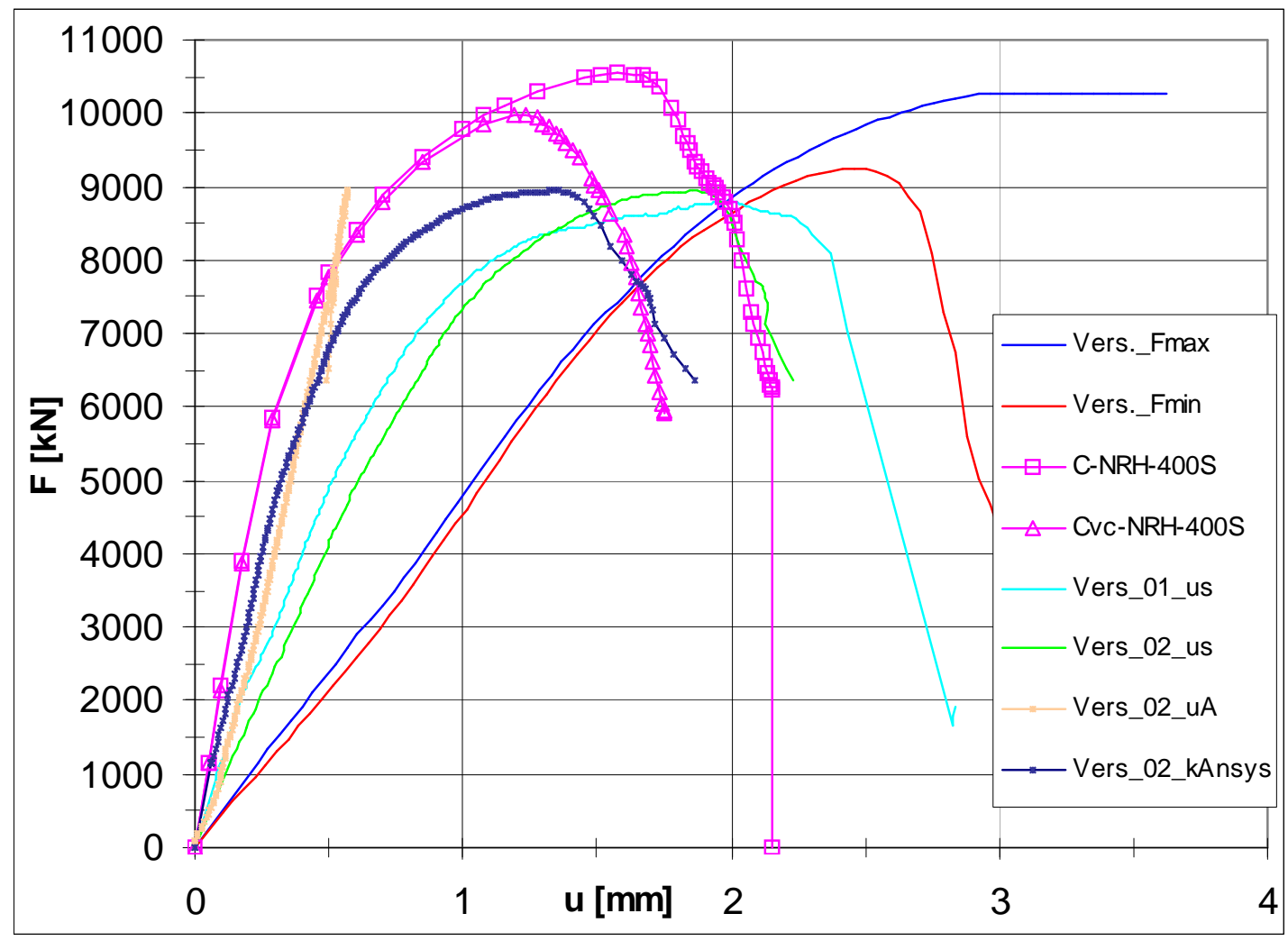

Diagramm 4.8: Versuche mit der Schraube Nr. 61=C=Cvc

Aus den Diagrammen zeigt sich, daß die Versuche, in denen die Relativverschiebung $u_{s}=0,5^{\star}\left(u_{s 1}+u_{s 2}\right)$ zwischen Schraubenschaft und Maschinenquerhaupt über den Verlauf der Prüfkraft gemessen wurde (Kurven „Vers_01_us“ und „Vers_02_us“) bereits Steifigkeiten aufweisen, die deutlich größer sind als bei den ursprünglichen Versuchen (Kurven „Vers._Fax“ und „Vers._Fmin“), bei denen die Nachgiebigkeiten des Schraubenkopfes in den Last-Verformungsbeziehungen enthalten sind. Die Versuche, in denen die Relativverschiebung $u_{A}=0,5^{\star}\left(u_{A 1}+u_{A 2}\right)$ zwischen der Auflagervorrichtung für die Unterkonstruktion und dem Maschinenquerhaupt gemessen wurde (Kurven „Vers_02_uA“) liefern für die Auflagervorrichtung eine Steifigkeit $\mathrm{k}_{\mathrm{A}} \approx 15 \mathrm{kN} / \mathrm{mm}$. Im Gegensatz hierzu wurde durch das FE-Modell eine Steifigkeit $\mathrm{k}_{\mathrm{A}, \mathrm{Ansys}}=200 \mathrm{kN} / \mathrm{mm}$ für diese Auflagervorrichtung unterstellt $-100 \mathrm{kN} / \mathrm{mm}$ für jeden gelagerten Querrand der Unterkonstruktion (vgl. Abschnitt 4.7). Die in den Diagrammen 4.7 und 4.8 dargestellten Kurven „Vers_02_kAnsys“ ergeben sich aus den Kurven „Vers_02_us“, in dem für diese Versuche die im FE-Modell unterstellte Auflagersteifigkeit von $200 \mathrm{kN} / \mathrm{mm}$ angenommen wurde. Hierfür wurden die Verschiebungen für eine Laststufe $F_{i}$ folgendermaBen umgerechnet:

$\mathrm{U}_{\text {Vers_02_kAnsys }}=\mathrm{u}_{\text {Vers_02_us }}-\mathrm{F}_{\mathrm{i}}^{*}\left(1 / \mathrm{k}_{\mathrm{A}}-1 / \mathrm{k}_{\mathrm{A}, \mathrm{Ansys}}\right)$

Da die so ermittelten Kurven „Vers_02_kAnsys“ gut übereinstimmen mit den LastVerformungsbeziehungen aus den FE-Berechnungen kann abschließend folgende Schlußfolgerung für das entwickelte FE-Modell festgehalten werden: 
Das FE-Modell ist in der Lage, die realen Auszugversuche sowohl hinsichtlich der Versagenslasten als auch hinsichtlich der relevanten Verformungsverhältnisse, die durch die Biegeverformung der Unterkonstruktion und durch Verformungen im Verzahnungsbereich zwischen Schrauben- und Mutergewinde bestimmt werden, gut wiederzugeben. 


\subsection{Parameterstudien}

\subsubsection{Bandbreite der Parameterstudien}

Zur Ableitung eines mechanischen Modells für die Auszugstragfähigkeit von gewindeformenden Schrauben wurden die in der Tabelle 4.9 aufgeführten Parameterstudien durchgeführt.

Die in diesen Parameterstudien untersuchten Randbedingungen für die Schrauben und die Unterkonstruktionen wurden unter Berücksichtigung der folgenden Gesichtspunkte festgelegt:

- Die zur Verifizierung und Kalibrierung des FE-Modells im Abschnitt 4.2, Tabelle 4.2 ausgewählten Schrauben wurden auch für die Parameterstudien herangezogen, da diese Schrauben hinsichtlich ihrer geometrischen Eigenschaften die Bandbreite der in der Leichtbauweise verwendeten Schrauben gut widerspiegeln. Sowohl die Gewindeaußendurchmesser $\left(4,64 \mathrm{~mm} \leq d_{g} \leq 6,35 \mathrm{~mm}\right.$, vgl. Tabelle 4.2) als auch die Gewindefeinheiten $\left(2,53 \leq d_{g} / P \leq 4,94\right.$, vgl. Tabelle 4.2) der Schrauben decken den für die Leichtbauweise gebräuchlichen Bereich ab. Darüber hinaus sind die sich aus unterschiedlichen Einsatzbereichen ergebenden Variationen der Überdeckung der Gewindegänge der Schrauben durch die Unterkonstruktion in den Parameterstudien dadurch berücksichtigt, daß durch die Schrauben für die Nennwerte der Gewindeaußendurchmesser 6,3mm, 5,5mm und 4,8mm jeweils drei unterschiedliche Verzahnungssituationen mit den Unterkonstruktionen (Variation der Werte gebildet aus der Differenz des Gewindeaußendurchmessers $d_{G}$ und des Kernlochdurchmessers $d_{L}$ in der Unterkonstruktion) untersucht werden. Für die gewindefurchende Schraube „F" sind durch die gewählten Kernlochdurchmesser $d_{L}$ in der Unterkonstruktion $\left(d_{L}=d_{K}=4,53 m m\right.$ für die Schraube „F1" und $d_{L}=5,00 m>d_{K}$ für die Schraube „F2“) zwei unterschiedliche Verzahnungssituationen durch die Parameterstudien berücksichtigt.

- Zur Untersuchung des Einflusses der Werkstoffestigkeit der Gewindegänge der Schrauben auf die Auszugstragfähigkeit wurden die Werkstoffgesetze „NRL“ (vgl. Anhang B, Diagramm B.16) und „CH“ (vgl. Anhang B, Diagramm B.18) für die Parameterstudien verwendet. Durch das Werkstoffgesetz „NRL“, das eine Zugfestigkeit von $680 \mathrm{~N} / \mathrm{mm}^{2}$ aufweist, werden die kleinsten Festigkeiten berücksichtigt, die aufgrund der Abschätzungen in der Tabelle 3.1 (Kapitel 3) sowie den Ausführungen im Abschnitt 4.5 im Gewindebereich von Schrauben aus nichtrostendem Stahl vorliegen. Das Werkstoffgesetz "CH“ weist eine Zugfestigkeit von $1250 \mathrm{~N} / \mathrm{mm}^{2}$ auf, die über dem in der Tabelle 3.1 abgeschätzten Mittelwert für die Zugfestigkeiten des Gewindekerns von Schrauben aus niedriglegier- 
ten Kohlestoffstählen liegt. Darüber hinaus liegt die Zugfestigkeit des Werkstoffgesetzes „ $\mathrm{CH}^{\prime}$ auch in etwa an der Obergrenze der Zugfestigkeit, die gemäß den Abschätzungen in der Tabelle $3.1 \mathrm{im}$ Gewindebereich von Schrauben aus nichtrostendem Stahl vorliegen kann. Durch die Werkstoffgesetze „NRL“ und „CH“ werden daher die möglichen Grenzbedingungen für die Werkstoffestigkeiten der in der Leichtbauweise verwendeten Schrauben gut wiedergegeben. Die im Abschnitt 4.5 festgestellte erhöhte Festigkeit auf den oberflächennahen Randbereichen der Gewindegängen von Schrauben aus niedriglegierten Kohlenstoffstählen wurde in den Parameterstudien nicht berücksichtigt, da die Ergebnisse der simulierten Versuchsserien gezeigt haben, daß diese erhöhte Festigkeit keinen signifikanten Einfluß auf die Auszugstragfähigkeit besitzt.

- Die Blechdicken der Unterkonstruktion wurden in den Parameterstudien innerhalb des Bereichs von $0,60 \mathrm{~mm}$ bis $4,90 \mathrm{~mm}$ variiert, da das entwickelte FE-Modell auch anhand von Versuchen verifiziert wurde, die mit Unterkonstruktionen dieses Blechdickenbereiches durchgeführt wurden. Die Schrauben „F1“, „B“ und „D“ weisen die für ihre jeweilige Gewindenenngröße größtmögliche Überdeckung ihrer Gewindegänge durch die Unterkonstruktion auf, da für diese Schrauben der in der Unterkonstruktion erzeugte Kernlochdurchmesser $d_{L}$ mit dem Kerndurchmesser $d_{K}$ des Schraubengewindes übereinstimmt. Der Einsatzbereich dieser Schrauben liegt daher in der Befestigung auf Unterkonstruktionen mit kleinen Blechdicken. Aus diesem Grund wurden in den Parameterstudien für diese Schrauben lediglich Blechdicken im Bereich zwischen 0,60mm und 3,06mm untersucht. Die Schrauben „J“ und „A“ weisen von allen für die Parameterstudien verwendeten Schrauben die kleinsten Überdeckungen ihrer Gewindegänge durch die Unterkonstruktion auf, da ihr Einsatzbereich in der Befestigung auf Unterkonstruktionen mit großen Blechdicken liegt. Daher wurden für diese Schrauben lediglich Blechdicken im Bereich zwischen 1,99mm und 4,90mm untersucht. Für alle übrigen Schrauben wurden die Blechdicken zwischen $0,60 \mathrm{~mm}$ und $4,90 \mathrm{~mm}$ variiert.

- Zur Untersuchung des Einflusses des Werkstoffverhaltens der Unterkonstruktion auf die Auszugstragfähigkeit wurden drei unterschiedliche Werkstoffgesetze untersucht. Stellvertretend für Unterkonstruktionen aus Aluminium wurde das im Anhang B im Diagramm B.11 dargestellte Werkstoffgesetz verwendet (die entsprechenden FE-Berechnungen der Parameterstudien sind mit der Bezeichnung „Al“ gekennzeichnet). Stellvertretend für einen Stahl, der aufgrund seiner Zugfestigkeit in die Festigkeitsklassen S235 und S280 gemäß DIN EN 10025-2 und DIN EN 10326 einzuordnen ist, wurde das im Anhang B im Diagramm B.1 dargestellte Werkstoffgesetz verwendet (die entsprechenden FEBerechnungen der Parameterstudien sind mit der Bezeichnung „S280“ gekennzeichnet). Zur Berücksichtigung der zukünftigen Entwicklungen im Leichtbau wurde stellvertretend für einen Stahl, der aufgrund seiner Zugfestigkeit in die Festigkeitsklassen S355 und 
S350 gemäß DIN EN 10025-2 und DIN EN 10326 einzuordnen ist, das im Anhang B im Diagramm B.12 dargestellte Werkstoffgesetz verwendet (die entsprechenden FEBerechnungen der Parameterstudien sind mit der Bezeichnung „S355“ gekennzeichnet). Die jeweiligen Werkstoffgesetze wurden in den FE-Berechnungen der Parameterstudien aufgrund der Ergebnisse der simulierten Versuchsserien zur Beschreibung des Werkstoffverhaltens der gesamten Unterkonstruktion - also sowohl für den Bereich des FEModells, in dem das Muttergewinde abgebildet wird, als auch für die Bereiche außerhalb des Muttergewindes - verwendet.

- Aufgrund der Ergebnisse der simulierten Versuchsserien wurde in allen FEBerechnungen der Parameterstudien ein Reibungskoeffizient von $\mu_{G}=0,35$ für die Gewindereibung zwischen den Schrauben- und Mutterflanken angenommen.

Tabelle 4.9: Randbedingungen für die Parameterstudien

\begin{tabular}{|c|c|c|c|c|c|}
\hline \multicolumn{4}{|c|}{ Schrauben } & \multicolumn{2}{|c|}{ Unterkonstruktionen } \\
\hline $\begin{array}{c}\text { Bezeichnung } \\
\text { vgl. Ab- } \\
\text { schnitt } 4.2, \\
\text { Tabelle } 4.2\end{array}$ & $\begin{array}{c}\text { Gewinde- } \\
\text { nenn } \varnothing \\
{[\mathrm{mm}]}\end{array}$ & $\begin{array}{l}\mathrm{d}_{\mathrm{g}}-\mathrm{d}_{\mathrm{L}} \\
{[\mathrm{mm}]}\end{array}$ & $\begin{array}{c}\text { Werkstoff- } \\
\text { gesetze }\end{array}$ & Blechdicken $\mathrm{t}_{\mathrm{K}}[\mathrm{mm}]$ & $\begin{array}{c}\text { Werkstoff- } \\
\text { gesetze }\end{array}$ \\
\hline $\mathrm{F} 1$ & \multirow[t]{2}{*}{6,5} & 1,82 & \multirow{12}{*}{$\begin{array}{l}\text { „NRL“ (vgl. } \\
\text { Anhang B, } \\
\text { Diagramm } \\
\text { B.16) }\end{array}$} & $\begin{array}{c}0,60 ; 0,69 ; 0,98 ; 1,47 ; \\
1,99 ; 3,06 ;\end{array}$ & \multirow{5}{*}{$\begin{array}{l}\text { „Al“ (vgl. An- } \\
\text { hang B, Dia- } \\
\text { gramm B.11) }\end{array}$} \\
\hline $\mathrm{F} 2$ & & 1,35 & & $\begin{array}{l}0,60 ; 0,69 ; 0,98 ; 1,47 ; \\
1,99 ; 3,06 ; 3,94 ; 4,90\end{array}$ & \\
\hline B & \multirow{3}{*}{6,3} & 1,39 & & $\begin{array}{c}0,60 ; 0,69 ; 0,98 ; 1,47 \\
1,99 ; 3,06 ;\end{array}$ & \\
\hline G & & 0,77 & & $\begin{array}{r}0,60 ; 0,69 ; 0,98 ; 1,47 \\
1,99 ; 3,06 ; 3,94 ; 4,90\end{array}$ & \\
\hline$J$ & & 0,60 & & $\begin{array}{c}1,99 ; 3,06 ; 3,50 ; 3,94 \\
4,50 ; 4,90\end{array}$ & \\
\hline $\mathrm{H}$ & \multirow{4}{*}{5,5} & 0,89 & & $\begin{array}{c}0,60 ; 0,69 ; 0,98 ; 1,47 \\
1,99 ; 3,06 ; 3,94 ; 4,90 \\
\end{array}$ & \multirow{4}{*}{$\begin{array}{c}\text { „S280“ (vgl. } \\
\text { Anhang B, Dia- } \\
\text { gramm B.1) }\end{array}$} \\
\hline C & & 0,64 & & $\begin{array}{c}0,60 ; 0,69 ; 0,98 ; 1,47 \\
1,99 ; 3,06 ; 3,94 ; 4,90\end{array}$ & \\
\hline Cve & & 0,64 & & $\begin{array}{c}0,60 ; 0,69 ; 0,98 ; 1,47 \\
1,99 ; 3,06 ; 3,94 ; 4,90\end{array}$ & \\
\hline A & & 0,30 & & $\begin{array}{c}1,99 ; 3,06 ; 3,50 ; 3,94 \\
4,50 ; 4,90\end{array}$ & \\
\hline D & & 1,38 & & $\begin{array}{c}0,60 ; 0,69 ; 0,98 ; 1,47 \\
1,99 ; 3,06 ;\end{array}$ & Anhang B, Dia- \\
\hline$E$ & 4,8 & 0,98 & & $\begin{array}{r}0,60 ; 0,69 ; 0,98 ; 1,47 \\
1,99 ; 3,06 ; 3,94 ; 4,90\end{array}$ & \\
\hline I & & 0,72 & & $\begin{array}{c}0,60 ; 0,69 ; 0,98 ; 1,47 ; \\
1,99 ; 3,06 ; 3,94 ; 4,90 \\
\end{array}$ & \\
\hline
\end{tabular}

Die in den 516 FE-Berechnungen der Parameterstudien erreichten Maximallasten sind im Anhang D in den Diagrammen D.1 bis D.12 dargestellt. 


\subsubsection{Entwicklung eines mechanischen Modells und formelmäßige Beschreibung der Auszugstragfähigkeit anhand der Ergebnisse der Parameterstudien}

\subsubsection{Versagensmodelle}

Zur Beschreibung der sich aus den Parameterstudien ergebenden Auszugstragfähigkeiten müssen drei Versagensmechanismen berücksichtigt werden. Die mechanischen Modelle, die diesen Versagensmechanismen zugrunde liegen, bilden den Ausgangspunkt für die formelmäßige Beschreibung der Auszugstragfähigkeit. Die Versagensmodelle werden nachfolgend erläutert.

\section{Versagen des Schraubengewindes}

Ein Versagensmechanismus ist dadurch gegeben, daß die Gewindegänge der Schraube durch das Muttergewinde abgeschert werden. Dieser Versagensmechanismus tritt dann auf, wenn die Tragfähigkeit und die Steifigkeit des Muttergewindes so groß sind, daß bis zum Versagen der Gewindegänge der Schraube keine nennenswerten Verformungen in den Gewindegängen des Muttergewindes auftreten. Für diesen Fall verlagern sich die Axialkomponenten der Kontaktspannungen zwischen dem Muttergewinde und dem Schraubengewinde mit zunehmender Laststeigerung aufgrund der größeren Biegenachgiebigkeit der im Verzahnungsbereich mit dem Muttergewinde weiter außen liegenden Bereiche der Gewindegänge der Schraube $\left(r>d_{\llcorner} / 2\right)$ sowie aufgrund der aus der geringeren Abscherfläche resultierenden geringeren Abschertragfähigkeiten dieser Bereiche immer weiter hin zu dem Radius, der dem Durchmesser $d_{L}$ des Kernloches in der Unterkonstruktion entspricht (Bild 4.19). Im Grenzzustand des Versagens werden die Kontaktkräfte zwischen dem Muttergewinde und dem Schraubengewinde fast ausschließlich im Schnitt $r=d_{L} / 2$ übertragen und die Gewindegänge der Schraube werden ausgehend von der Stelle, die auf der oberen Gewindeflanke im Schnitt $r=d_{L} / 2$ liegt, abgeschert. 


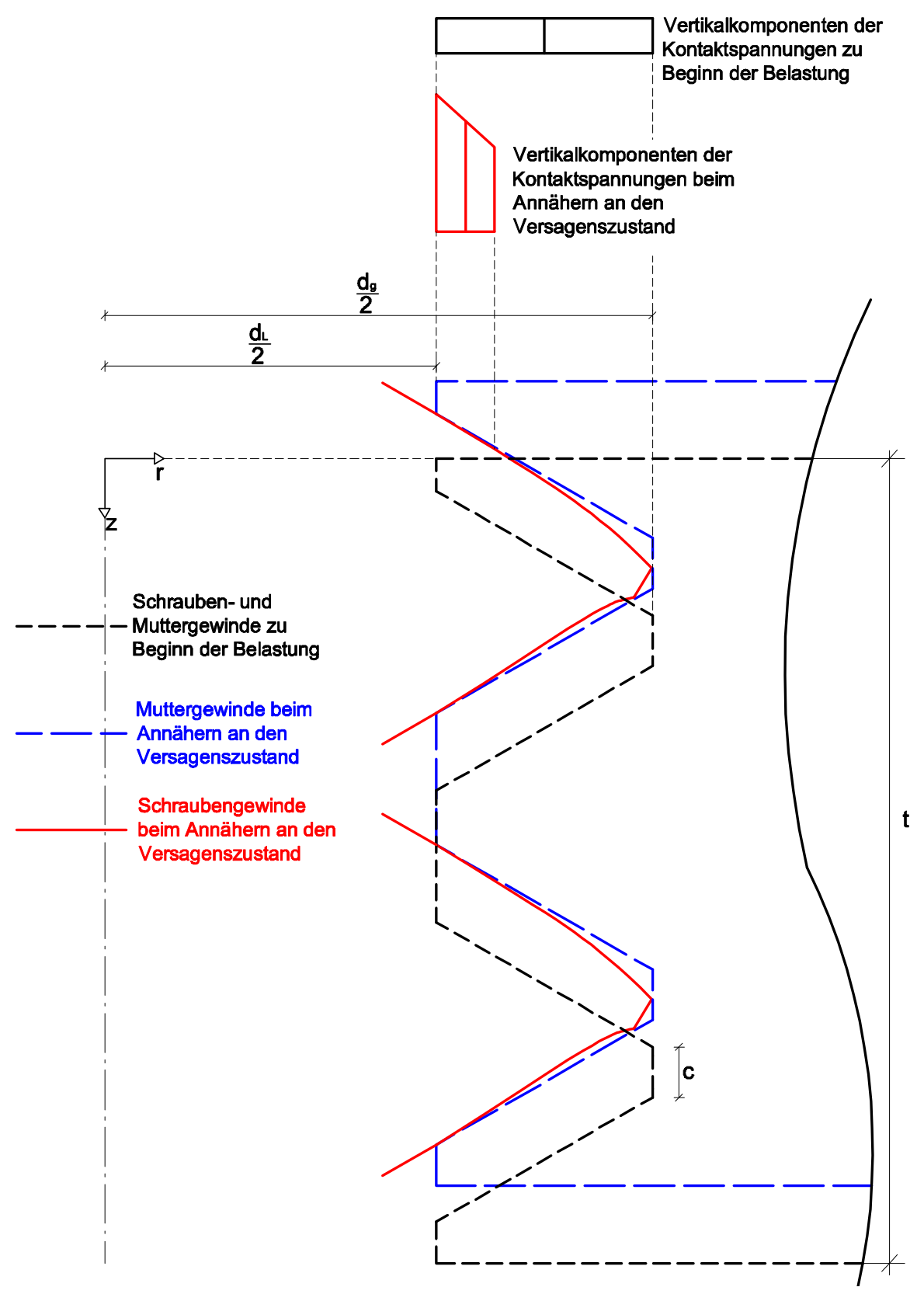

Bild 4.19: Verformungen und Kontaktspannungen im Verzahnungsbereich zwischen Schrauben- und Muttergewinde für ein Versagen des Schraubengewindes

Die aus diesem Versagensmechanismus resultierende Auszugstragfähigkeit ergibt sich aus der Multiplikation der Scherfestigkeit der Gewindegänge der Schraube mit der maßgebenden Abscherfläche in den Gewindegängen der Schraube. Zur Berechnung der Abscherfläche wurde der Verzahnungsbereich zwischen dem Schraubengewinde und dem Muttergewinde in den Bildern 4.20 und 4.21 folgendermaßen veranschaulicht:

- Das Bild 4.20 zeigt eine Ansicht der Schraube mit einem axialen Schnitt durch das Muttergewinde (Bild 4.20 a)) sowie den zu dieser Ansicht gehörenden axialen Schnitt durch die Schraube und das Muttergewinde (Bild 4.20 b)). Die Kontaktkräfte zwischen dem Schraubengewinde und dem Muttergewinde können zwischen der oberen Gewindeflanke der Schraube und dem Muttergewinde auf dem Bereich $d_{\llcorner} / 2 \leq r \leq d_{g} / 2$ übertragen werden. 
- Mit Hilfe des Bildes 4.21 wird der Verlauf des Verzahnungsbereiches zwischen dem Schraubengewinde und dem Muttergewinde in Umfangsrichtung verdeutlicht. Die Lage der Koordinaten $r$ und $z$ wird aus dem Bild 4.20 b) deutlich. Die Koordinate $z$ verläuft in Richtung der Schraubenachse. Sie besitzt auf der Höhe der Oberkante der Unterkonstruktion den Wert $\mathrm{z}=0$ und auf der Höhe der Unterkante der Unterkonstruktion den Wert der Blechdicke der Unterkonstruktion $(z=t)$. Die Koordinate $r$ verläuft in radialer Richtung und die Koordinate $\varphi$ beschreibt die Umfangsrichtung. Für $\varphi=0$ wird derjenige Schnitt beschrieben, in dem der Punkt $\mathrm{P}_{1}$ liegt, an dem die obere Gewindeflanke der Schraube an der Stelle $r=d_{g} / 2$ gerade in die Unterkonstruktion einläuft.

- Die Ansicht im Bild 4.21 gibt die Abwicklung der Gewindegänge der Schraube in den Schnitten $r=d_{L} / 2$ (rot gezeichnete Linien) und $r=d_{g} / 2$ (blau gezeichnete Linien) über die Blechdicke der Unterkonstruktion wieder. Der Verlauf der oberen Gewindeflanke der Schraube ist durch die für die Schnitte $r=d_{L} / 2$ und $r=d_{g} / 2$ durchgezogen gezeichneten Linien gekennzeichnet, und der Verlauf der unteren Gewindeflanke der Schraube ist durch die für die Schnitte $r=d_{\llcorner} / 2$ und $r=d_{g} / 2$ gestrichelt gezeichneten Linien gekennzeichnet. Die Neigung $y$ dieser Linien gegenüber der Horizontalen (Umfangsrichtung $\varphi$ ) ergibt sich aufgrund der Gewindesteigung $P$ der Schraube aus tan $Y=P /\left(2^{*} \pi\right)$. Die Höhendifferenz dieser Linien an einer beliebigen Stelle $\varphi$ ergibt sich mit der Gewindeabflachung $c$ und dem Flankenwinkel a der Schraube aus dem Schnitt $A-A$. In den Punkten $P_{1}$ und $P_{2}$ läuft die obere Gewindeflanke der Schraube in den Schnitten $r=d_{L} / 2$ und $r=d_{g} / 2$ gerade in die Unterkonstruktion ein. In den Punkten $\mathrm{P}_{3}$ und $\mathrm{P}_{4}$ läuft die obere Gewindeflanke der Schraube in den Schnitten $r=d_{L} / 2$ und $r=d_{g} / 2$ gerade aus der Unterkonstruktion aus. Die Trapezfläche mit den Begrenzungspunkten $\mathrm{P}_{1}$ bis $\mathrm{P}_{4}$ stellt somit den Bereich dar, in dem die obere Gewindeflanke der Schraube durch das Muttergewinde überdeckt wird. Die Überdeckung der oberen Gewindeflanke durch das Muttergewinde erstreckt sich in den Schnitten $r=d_{\llcorner} / 2$ und $r=d_{g} / 2$ über den Winkel $\varphi_{t}=t^{\star} 2^{*} \pi / P$. Der Ein- und Auslauf der oberen Gewindeflanke der Schraube im Schnitt $r=d_{g} / 2$ ist gegenüber dem Ein- und Auslauf der oberen Gewindeflanke der Schraube im Schnitt $r=d_{\llcorner} / 2$ aufgrund der Höhendifferenz $\Delta z_{1}$ und aufgrund der Gewindesteigung $P$ um die Winkeldifferenz $\varphi_{E A}=\left(d_{g}-d_{L}\right)^{*} \tan (\alpha / 2)^{*} \pi / P$ verschoben.

- Im Schnitt B-B ist die Draufsicht auf den Verlauf der oberen Gewindeflanke der Schraube in Umfangsrichtung dargestellt. Durch die strichpunktiert umrandete Trapezfläche (Begrenzungspunkte $\mathrm{P}_{1}$ bis $\mathrm{P}_{4}$ ) wird aus diesem Bild der radiale Verlauf der Verzahnung zwischen der oberen Gewindeflanke der Schraube und dem Muttergewinde deutlich. 


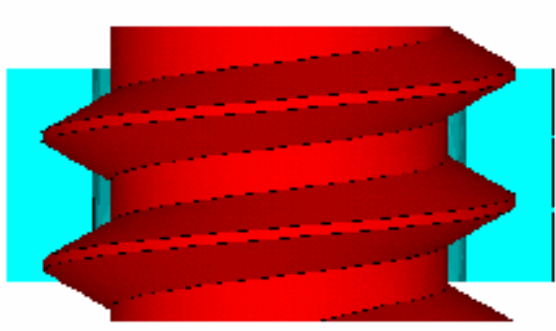

a)

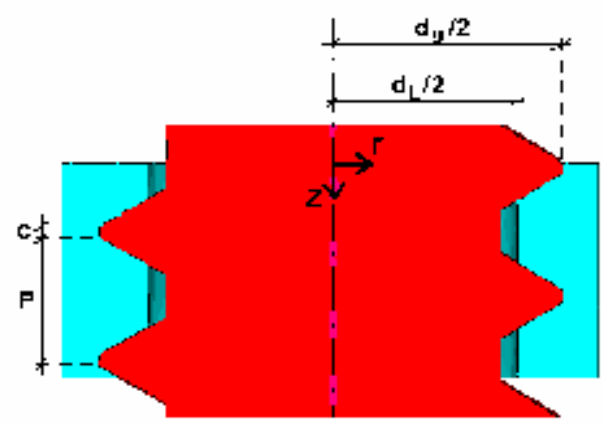

b)

Bild 4.20: a) Ansicht der Schraube mit axialem Schnitt durch das Muttergewinde; b) axialer Schnitt durch die Schraube und das Muttergewinde

\section{Ansicht über die Blechdicke:}

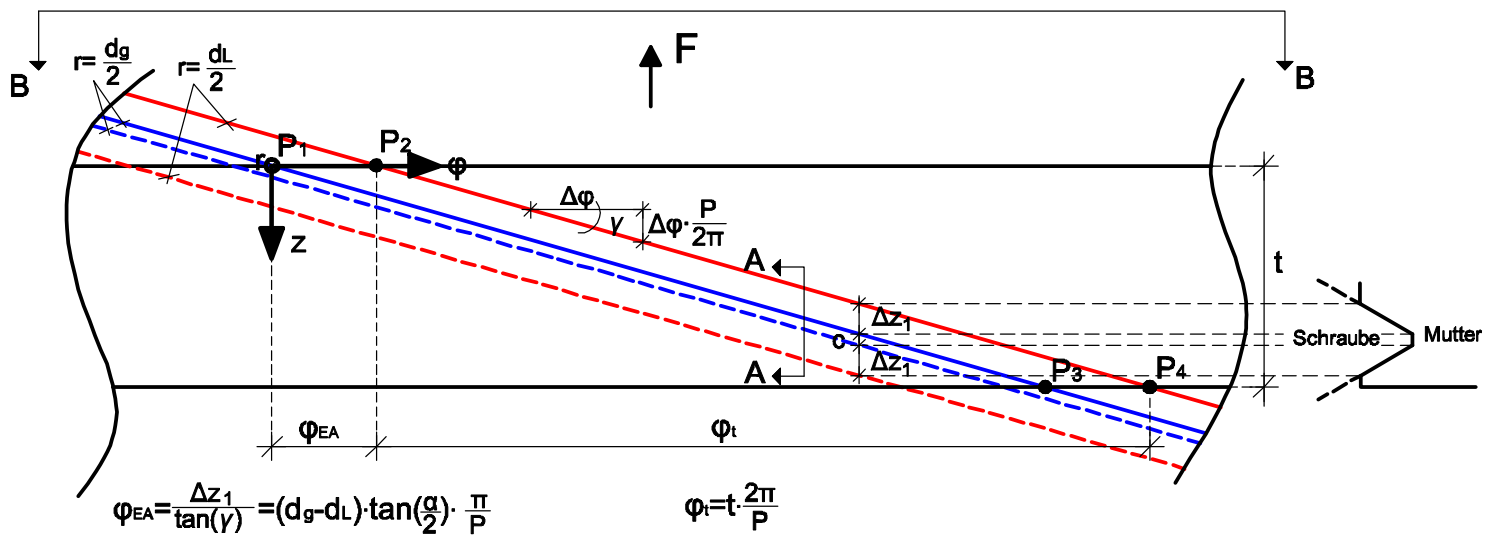

Schnitt A-A: (vergrößerter Maßstab)

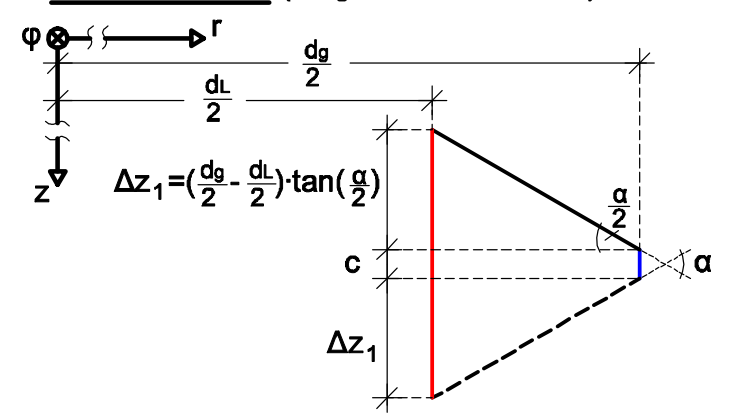

Schnitt B-B: Draufsicht auf die obere Gewindeflanke der Schraube

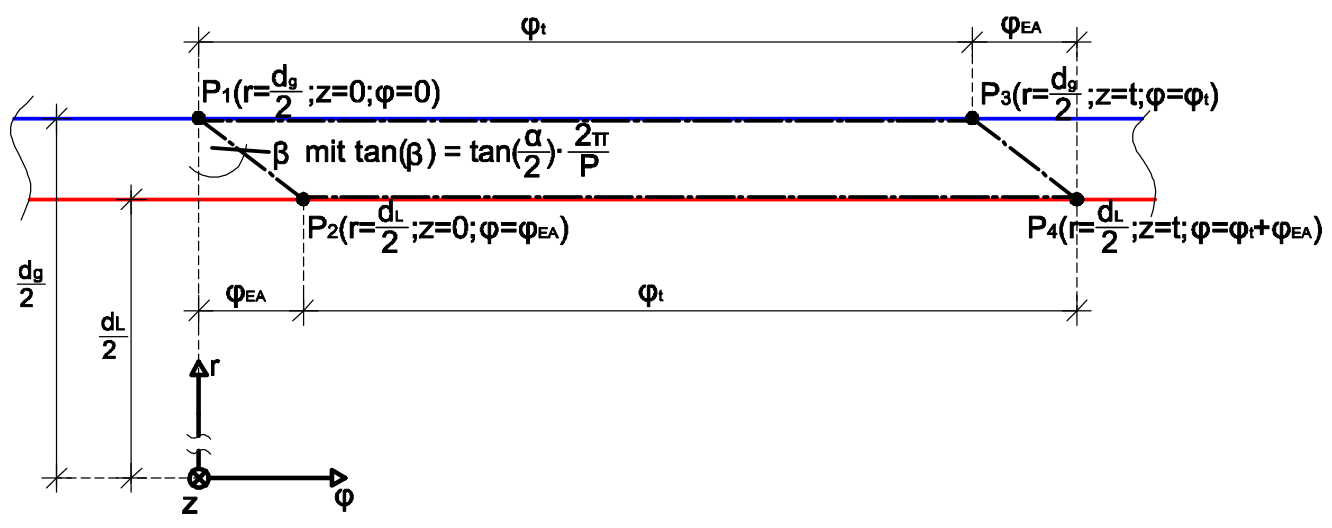

Bild 4.21: Verlauf des Verzahnungsbereiches zwischen dem Schraubengewinde und dem Muttergewinde in Umfangsrichtung 
Als eine Abschätzung für die maßgebende Abscherfläche, die aus dem oben beschriebenen Versagensmechanismus resultiert, wurde diejenige Fläche $A_{S, S G}$ herangezogen, die in den Gewindegängen der Schraube im Schnitt $r=d_{L} / 2$ auf dem Bereich vorliegt, auf dem Kontaktkräfte zwischen dem Schrauben und dem Muttergewinde auftreten können (Bereich $\varphi_{E A} \leq \varphi \leq \varphi_{t}+\varphi_{E A}$ im Bild 4.21). Durch diese Abscherfläche wird die tatsächlich für das Versagen maßgebende Abscherfläche unterschätzt, da sich aufgrund der Lastausbreitung auch Bereiche in den Gewindegängen der Schraube an der Lastabtragung beteiligen, die vor allem in radialer Richtung aber auch in zirkularer Richtung über den durch die Fläche $A_{S, S G}$ abgedeckten Bereich hinausgehen können.

Die Fläche $A_{S, S G}$ ergibt sich mit Hilfe der Darstellung im Bild 4.21 aus folgender Beziehung:

$$
\begin{aligned}
A_{S, S G} & =\left(c+2^{*} \Delta z_{1}\right)^{*}\left(d_{L} / 2\right)^{*} \varphi_{t} \\
& =\left[c+\left(d_{g}-d_{L}\right)^{*} \tan (\alpha / 2)\right]^{*} d_{L}{ }^{*} t^{*} \pi / P
\end{aligned}
$$

Mit der Fläche $A_{S, S G}$ und der Scherfestigkeit $\tau_{m, S G}$ der Gewindegänge der Schraube ergibt sich eine konservative Abschätzung der Auszugstragfähigkeit für den Versagensfall des Abscherens der Gewindegänge der Schraube $\mathrm{F}_{\mathrm{As}, \mathrm{SG}}^{\star}$ aus:

$\mathrm{F}_{\mathrm{As}, \mathrm{SG}}^{*}=\tau_{\mathrm{m}, \mathrm{SG}}{ }^{*} \mathrm{~A}_{\mathrm{S}, \mathrm{SG}}=\tau_{\mathrm{m}, \mathrm{SG}}{ }^{*}\left[\mathrm{C}+\left(\mathrm{d}_{\mathrm{g}}-\mathrm{d}_{\mathrm{L}}\right)^{*} \tan (\alpha / 2)\right]^{*} \mathrm{~d}_{\mathrm{L}}{ }^{*} \mathrm{t}^{*} \pi / \mathrm{P}$

Zur Kalibrierung der Beziehung (4.6) anhand der Ergebnisse der Parameterstudien wurden für die Scherfestigkeiten $\tau_{\mathrm{m}, \mathrm{SG}}$ aufgrund der von Mises Hypothese, über die in den FE-

Berechnungen die Vergleichsspannungen berechnet werden, die mit $\frac{1}{\sqrt{3}}=0,577$ multiplizierten Werte der wahren Zugfestigkeiten der Werkstoffgesetzte „NRL“ und „CH“ eingesetzt

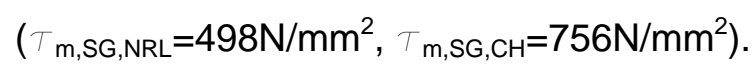

\section{Versagen des Muttergewindes}

Dieser zweite Versagensmechanismus ist dadurch bestimmt, daß die Tragfähigkeit und die Steifigkeit der Gewindegänge der Schraube so groß sind, daß der oben beschriebene erste Versagensmechanismus nicht auftritt und in den Gewindegängen der Schraube bis zum Versagen des Muttergewindes keine nennenswerten Verformungen stattfinden. Genau entgegengesetzt zu den Verhältnissen beim ersten Versagensmechanismus verlagern sich in diesem Fall die Vertikalkomponenten der Kontaktspannungen zwischen dem Muttergewinde und dem Schraubengewinde mit zunehmender Laststeigerung aufgrund der größeren Biegenachgiebigkeit der im Verzahnungsbereich mit dem Schraubengewinde weiter innen liegenden Bereiche der Gewindegänge des Muttergewindes $\left(r<d_{g} / 2\right)$ sowie aufgrund der aus der geringeren Abscherfläche resultierenden geringeren Abschertragfähigkeiten dieser Be- 
reiche immer weiter hin zu dem Radius, der dem Außendurchmesser $d_{g}$ der Schraube entspricht (Bild 4.22).

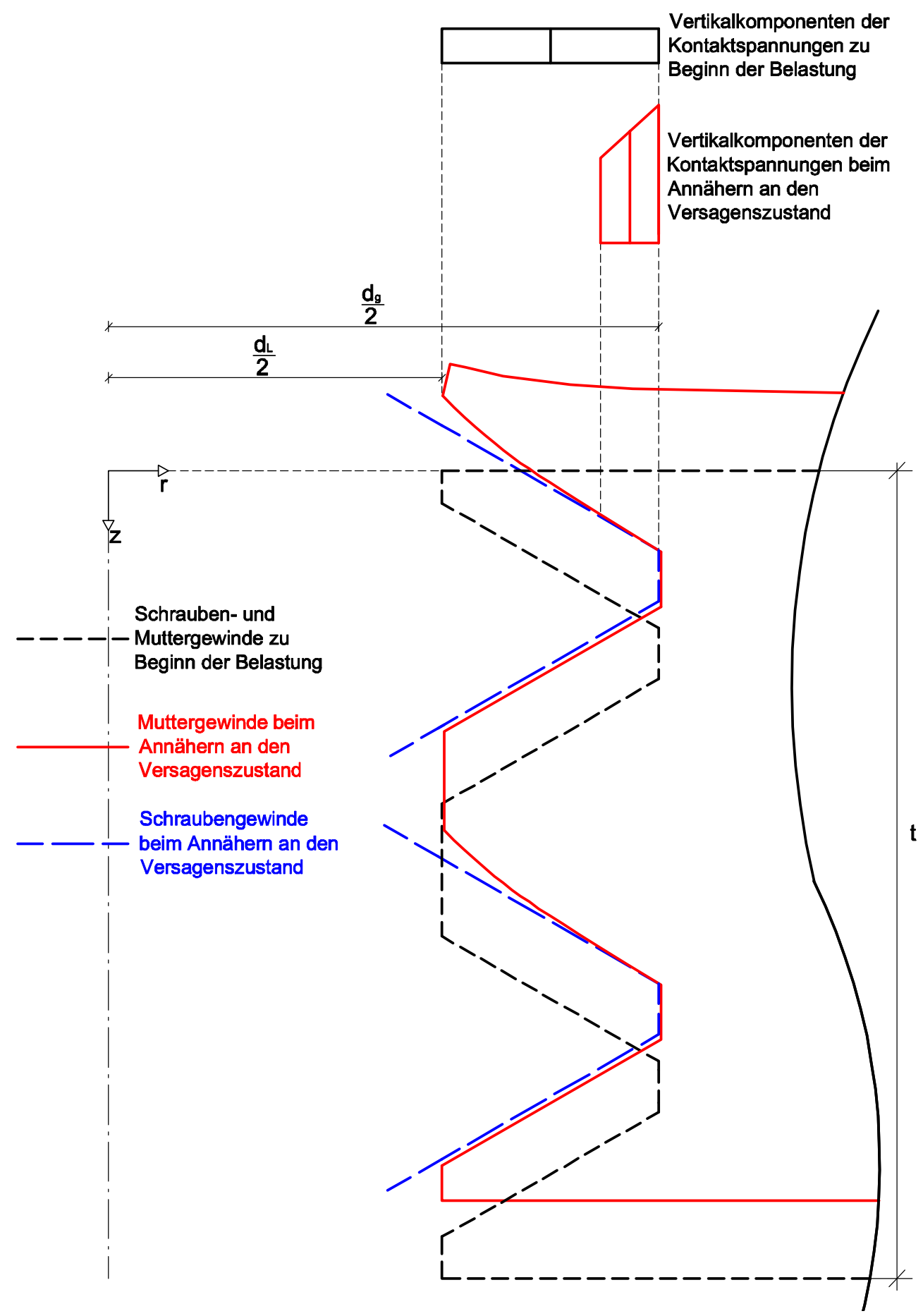

Bild 4.22: Verformungen und Kontaktspannungen im Verzahnungsbereich zwischen Schrauben- und Muttergewinde für ein Versagen des Muttergewindes

Unter der Voraussetzung, daß die Steifigkeit des Muttergewindes so groß ist, daß keine radiale Aufweitung des Mutterkörpers stattfindet, ergibt sich eine Grenzsituation für das Versagen, das durch das Muttergewinde bestimmt wird, daraus, daß die in radialer Richtung nicht aufgeweiteten Gewindegänge des Muttergewindes im Verzahnungsbereich mit dem Schraubengewinde im Schnitt $r=d_{g} / 2$ abgeschert werden. Sofern es aufgrund der Radial- 
komponenten der Kontaktkräfte zu einer radialen Aufweitung des Mutterkörpers kommt, reduziert sich die durch die erste Grenzsituation beschriebene Auszugstragfähigkeit aus folgenden Gründen:

- Durch die radiale Aufweitung weist das Muttergewinde im Schnitt $r=d_{g} / 2$, in dem sich die Vertikalkomponenten der Kontaktkräfte im Grenzzustand des Versagens konzentrieren, eine gegenüber dem nicht aufgeweiteten Muttergewinde reduzierte Gewindehöhe auf (Bild 4.23). Die Abschertragfähigkeit des radial aufgeweiteten Muttergewindes ist daher gegenüber dem nicht aufgeweitetem Muttergewinde reduziert.

- Für den Fall, daß aufgrund eines großen Bohrspitzen- oder Vorbohrdurchmessers nur eine relativ geringe Verzahnung $d_{g}-d_{L}$ zwischen dem Muttergewinde und dem Schraubengewinde vorliegt, kann die radiale Mutteraufweitung im Extremfall dazu führen, daß im oberen Bereich der Unterkonstruktion, in dem die radiale Aufweitung des Mutterkörpers durch radiale und zirkulare Zugspannungen verstärkt wird, die aus der Biegtragwirkung der Unterkonstruktion resultieren, die Verzahnung zwischen Schrauben- und Muttergewinde ganz verloren geht und somit in diesem Bereich die Gewindegänge des Muttergewindes nicht abgeschert werden müssen.

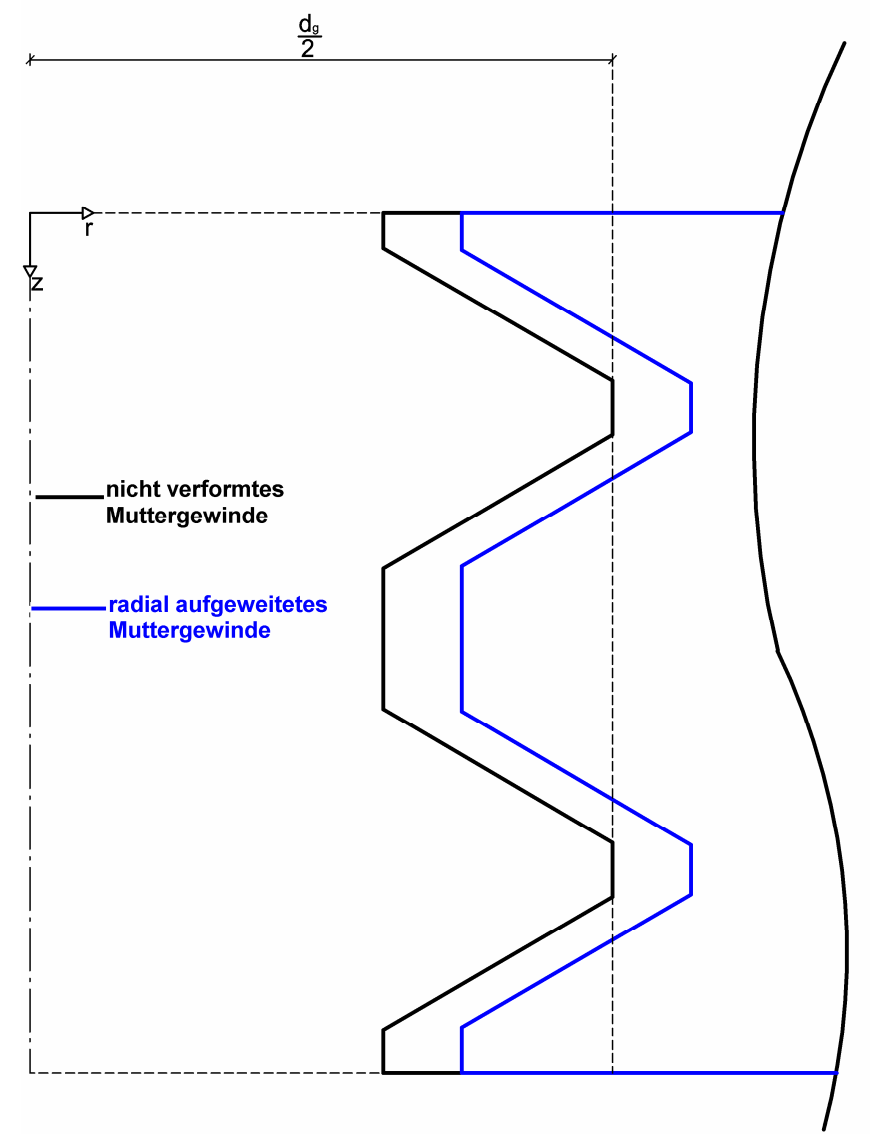

Bild 4.23: Reduzierte Abschertragfähigkeit durch eine radiale Aufweitung des Muttergewindes 
Für die Grenzsituation, daß der Mutterkörper nicht radial aufgeweitet ist, müssen zur Berechnung der maßgebenden Abscherfläche im Muttergewinde in Abhängigkeit von der Blechdicke der Unterkonstruktion und der Schraubengeometrie drei Fälle unterschieden werden, die in den Bildern 4.24 bis 4.26 dargestellt sind. In diesen Bildern wurde zur Veranschaulichung des Verzahnungsbereiches zwischen dem Schraubengewinde und dem Muttergewinde eine zum Bild 4.21 analoge Darstellung gewählt. Gegenüber dem Bild 4.21, welches zur Berechnung der Abscherfläche $A_{S, S G}$ herangezogen wurde, ist in den Bildern 4.24 bis 4.26 noch der Bereich auf der oberen Gewindeflanke der Schraube grün markiert, in dem das Schraubengewinde vom Lochrand her bis zur vollen Gewindetiefe einläuft $\left(\varphi_{\mathrm{I}} \leq \varphi \leq \varphi_{\mathrm{t}}+\varphi_{\text {EA }}\right)$. Berücksichtigt man auch die gemäß Bild 4.8 vor dem Eintritt stehende Kontaktfläche des Schraubengewindes bei der Abschätzung für die maßgebende Abscherfläche für den Fall, daß das Muttergewinde nicht radial aufgeweitet ist, so ergibt sich die Fläche $A_{S, M G}$, die in den Gewindegängen des Muttergewindes im Schnitt $r=d_{g} / 2$ auf dem gesamten Bereich $0 \leq \varphi \leq \varphi_{\mathrm{t}}+\varphi_{\text {EA }}$ vorliegt. Diese Fläche ist in den Bildern 4.24 bis 4.26 grau gekennzeichnet. Der Schnitt $r=d_{g} / 2$ auf dem gesamten Bereich $0 \leq \varphi \leq \varphi_{t}+\varphi_{E A}-$ also auch auf dem Bereich $\varphi_{\mathrm{t}} \leq \varphi \leq \varphi_{\mathrm{t}}+\varphi_{\mathrm{EA}}$, auf dem zwischen der oberen Gewindeflanke der Schraube und der Unterkonstruktion im Schnitt $r=d_{g} / 2$ zu Beginn der Belastung zunächst gar kein Kontakt besteht - wurde aus folgenden Gründen gewählt:

- Die Kontaktkräfte verlagern sich mit zunehmender Laststeigerung im Bereich $0 \leq \varphi \leq \varphi_{t}$ hin zu dem Schnitt $r=d_{g} / 2$ und im Auslauf des Muttergewindes (Bereich $\varphi_{t} \leq \varphi \leq \varphi_{t}+\varphi_{\text {EA }}$ ) verlagern sie sich hin zu der Linie, die in den Bildern 4.24 bis 4.26 als Verbindungsgerade der Punkte $\mathrm{P}_{3}$ und $\mathrm{P}_{4}$ eingezeichnet ist. Aufgrund von plastischen Verformungen, die sich im Muttergewinde durch das Erreichen der Abschertragfähigkeit in den Gewindegängen entlang der Linie $\mathrm{P}_{1}-\mathrm{P}_{3}-\mathrm{P}_{4}$ einstellen, kann es ebenfalls zu einem Kontakt zwischen den in den Bildern 4.24 bis 4.26 grün markierten Bereich der oberen Gewindeflanke der Schraube und der Unterseite der Unterkonstruktion kommen. Dies rechtfertigt die Annahme, daß für den gesamten Bereich $0 \leq \varphi \leq \varphi_{\mathrm{t}}+\varphi_{\text {EA }}$ der Schnitt $r=d_{g} / 2$ zur Berechnung der maßgebenden Abscherfläche $A_{S, M G}$ herangezogen wird.

- Die Abscherfläche $A_{S, M G}$, die sich im Muttergewinde im Schnitt $r=d_{g} / 2$ auf dem Bereich $0 \leq \varphi \leq \varphi_{t}+\varphi_{E A}$ ergibt, ist gegenüber der Abscherfläche entlang der Linie $P_{1}-P_{3}-P_{4}$ für die in den Parameterstudien untersuchten Randbedingungen nur um maximal 10\% größer - die größten Abweichungen ergeben sich für die kleinste Blechdicke $t=0,60 \mathrm{~mm}$, da für diese Blechdicke das Verhältnis $\varphi_{\mathrm{EA}} / \varphi_{\mathrm{t}}$ am größten wird.

- Die Abscherfläche $A_{S, M G}$ führt aufgrund des konstant angenommenen Radiuses $r=d_{g} / 2$ gegenüber der Abscherfläche entlang der Linie $\mathrm{P}_{1}-\mathrm{P}_{3}-\mathrm{P}_{4} \mathrm{zu}$ einer vereinfachten formelmäßigen Beschreibung der Auszugstragfähigkeit. 


\section{Ansicht über die Blechdicke:}

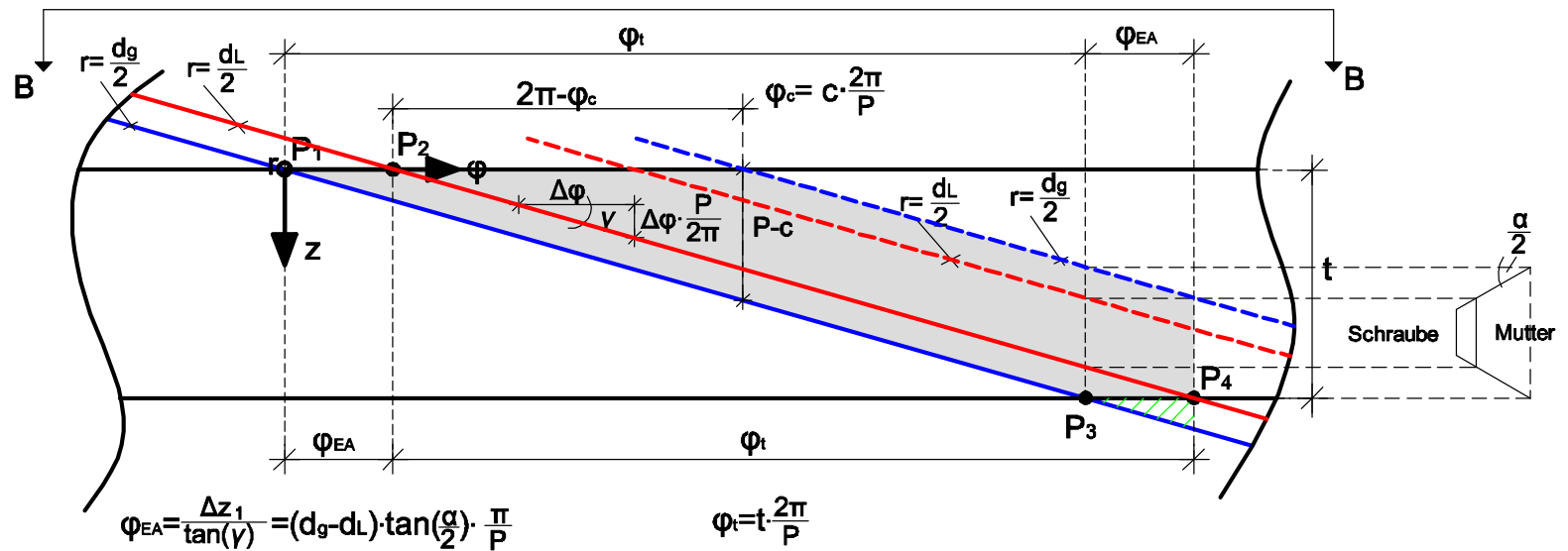

Schnitt B-B: Scherflächenverlauf in der Abwicklung im Grundriß

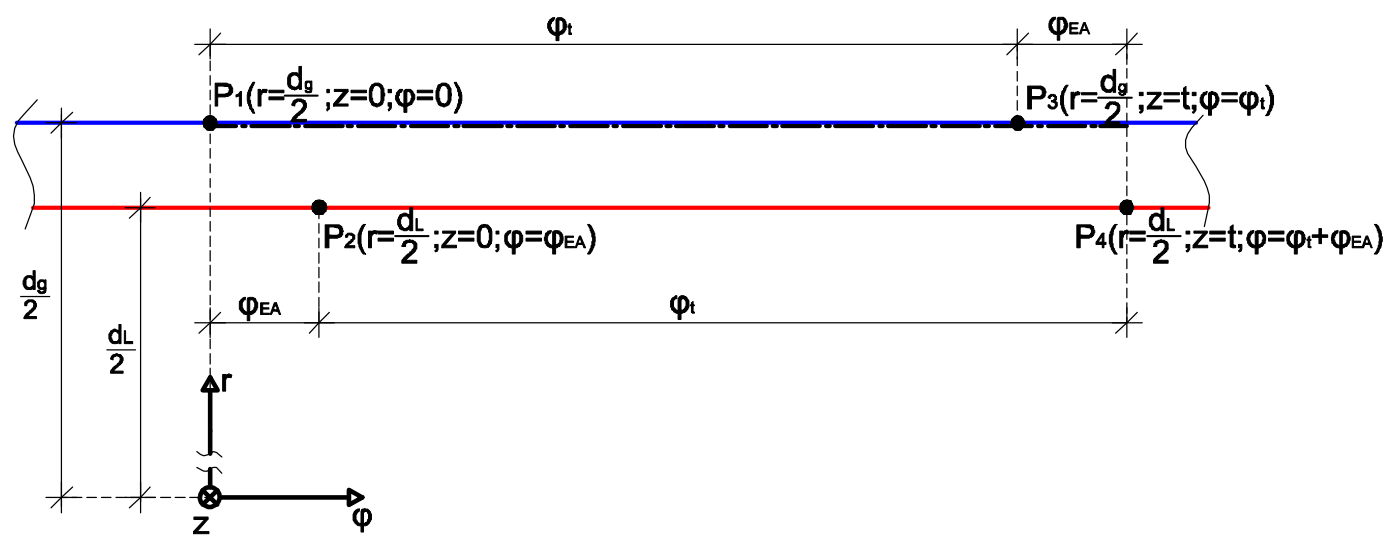

Bild 4.24: Abscherfläche $A_{S, M G}$ für den Fall I: $t \geq P-c$ Û $\varphi_{t} \geq 2 \pi-\varphi_{c}$ 


\section{Ansicht über die Blechdicke:}

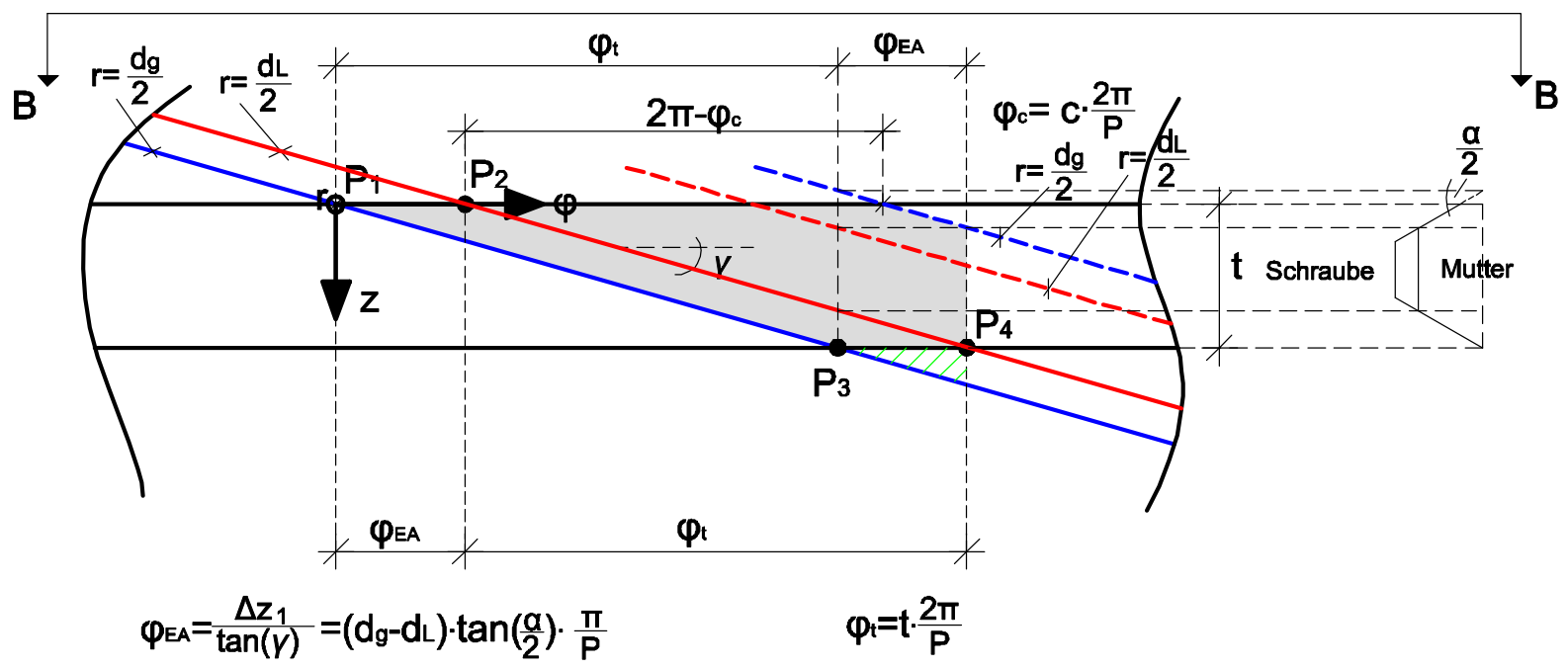

Schnitt B-B: Scherflächenverlauf in der Abwicklung im Grundriß

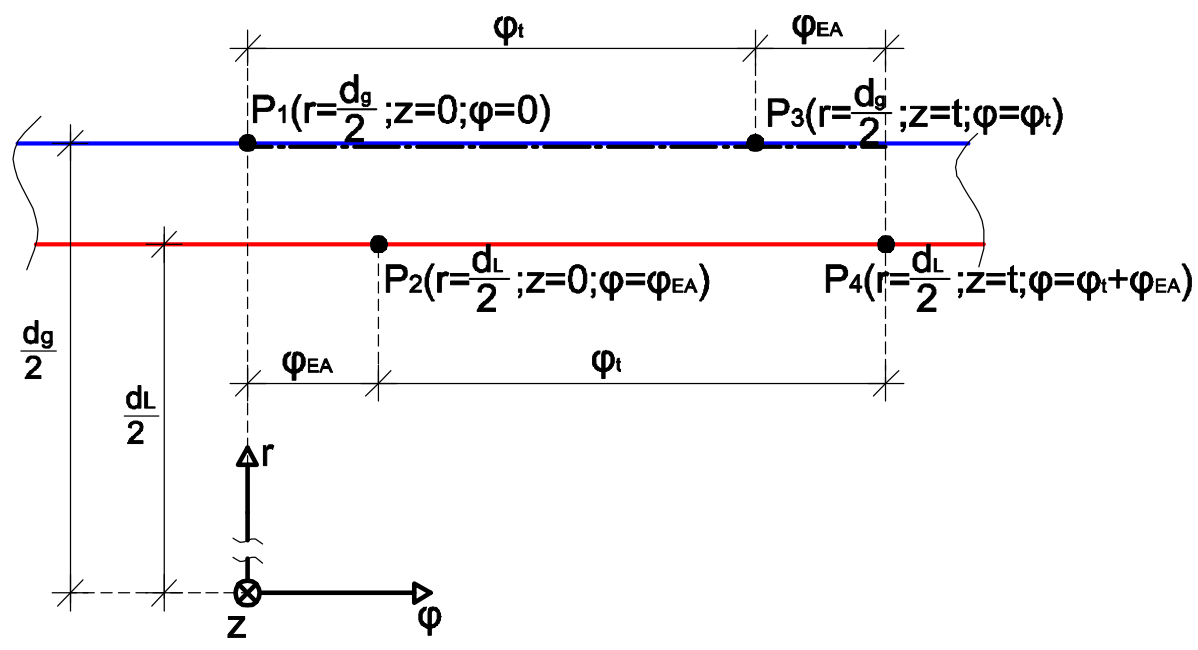

Bild 4.25: Abscherfläche $A_{S, M G}$ für den Fall II:

$\varphi_{\mathrm{t}}<2 \pi-\varphi_{\mathrm{c}}$ und $\varphi_{\mathrm{t}}+\varphi_{\mathrm{EA}}>2 \pi-\varphi_{\mathrm{c}}$ Û $2 \pi-\varphi_{\mathrm{c}}-\varphi_{\mathrm{EA}}<\varphi_{\mathrm{t}}<2 \pi-\varphi_{\mathrm{c}}$ 


\section{Ansicht über die Blechdicke:}

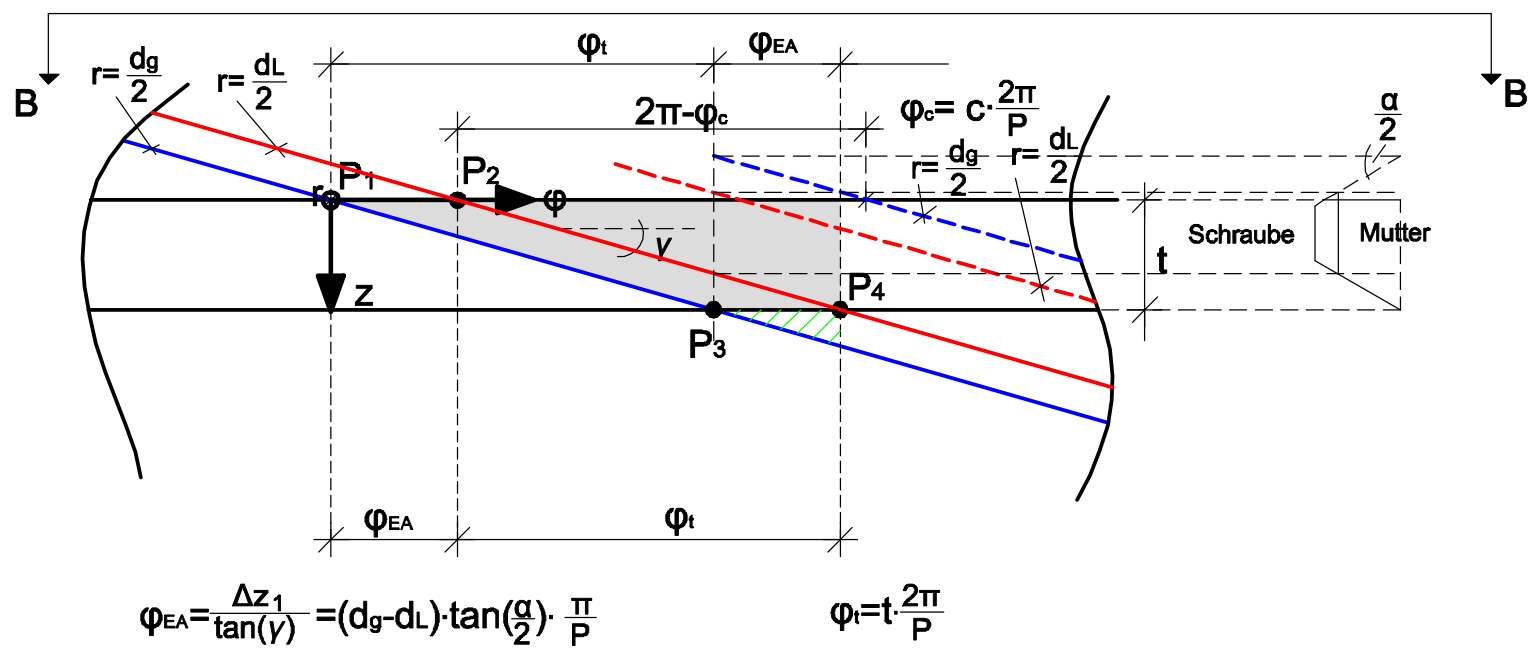

Schnitt B-B: Scherflächenverlauf in der Abwicklung im Grundriß

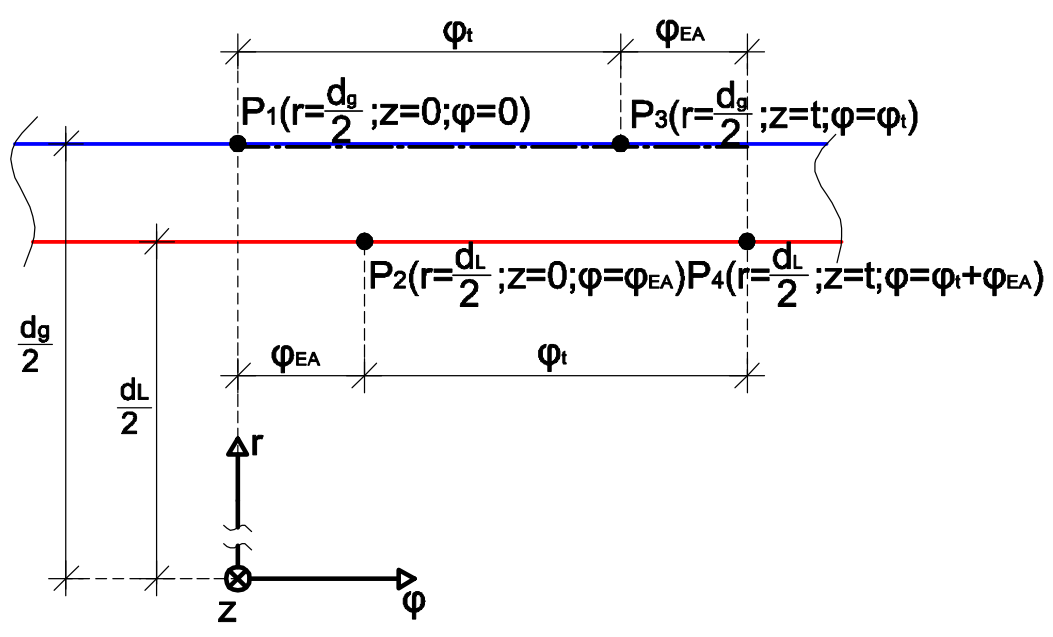

Bild 4.26: Abscherfläche $A_{S, M G}$ für den Fall III: $\varphi_{t}+\varphi_{E A} \leq 2 \pi-\varphi_{c} \hat{U} \quad \varphi_{t} \leq 2 \pi-\varphi_{c}-\varphi_{E A}$ 
Die Fläche $A_{S, M G}$ ergibt sich mit Hilfe der Darstellungen in den Bildern 4.24 bis 4.26 und der nachfolgenden Erläuterungen aus den Beziehungen (4.7) bis (4.9).

- Der im Bild 4.24 dargestellte Fall ist dadurch festgelegt, daß die Dicke der Unterkonstruktion so groß ist, daß im Muttergewinde im Schnitt $r=d_{g} / 2$ die maximal mögliche Gewindehöhe $h_{\max }=P-c$ erreicht wird. Die Bedingung hierfür lautet $t \geq P-c$ (vgl. auch Bild 4.27), die sich mit $\varphi_{\mathrm{t}}=\mathrm{t} / \mathrm{P}^{*} 2 \pi$ und $\varphi_{\mathrm{c}}=\mathrm{c} / \mathrm{P}^{*} 2 \pi$ auch durch $\varphi_{\mathrm{t}} \geq 2 \pi-\varphi_{\mathrm{c}}$ ausdrücken läßt. Für diesen Fall entspricht die Gewindehöhe des Muttergewindes im Schnitt $r=d_{g} / 2$ bis zu der Stelle $\varphi \leq 2 \pi-\varphi_{c}$ der im Bild 4.24 durch die Koordinate $z$ beschriebenen Höhe der oberen Gewindeflanke der Schraube im Schnitt $r=d_{g} / 2\left(h=z=\varphi^{*} P /\left(2^{*} \pi\right)\right)$. An der Stelle $\varphi=2 \pi-\varphi_{c}$ ist die maximal mögliche Gewindehöhe $h_{\max }=P-c$ erreicht, die bis zum Beginn des Auslauf des Muttergewindes $\varphi=\varphi_{\mathrm{t}}$ beibehalten wird. Im Auslauf des Muttergewindes $\varphi_{\mathrm{t}} \leq \varphi \leq \varphi_{\mathrm{t}}+\varphi_{\text {EA }}$ wird diese Höhe $h_{\max }$ aufgrund der konstanten Höhe der Blechunterkante $(z=t)$ reduziert $\left(h_{\text {Auslauf }}=P-c-\left(\varphi-\varphi_{t}\right)^{*} P /\left(2^{*} \pi\right)\right)$. Aus diesen Überlegungen ergibt sich die Abscherfläche $A_{S, M G}$ für den Fall $\varphi_{\mathrm{t}} \geq 2 \pi-\varphi_{\mathrm{c}}$ aus der Beziehung (4.7).

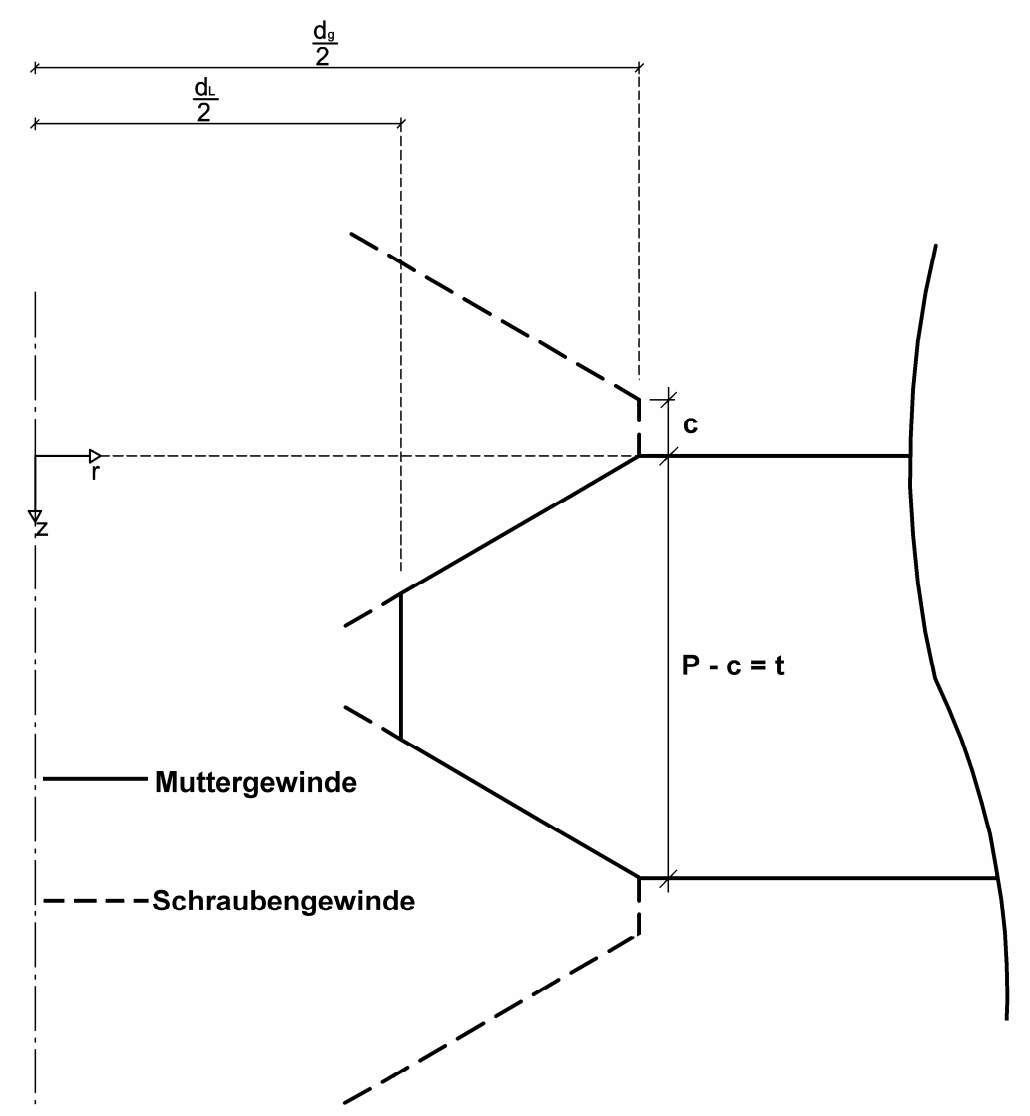

Bild 4.27: Grenzdicke der Unterkonstruktion, bei der sich gerade ein vollständiger Gewindezahn in der Unterkonstruktion ausbilden kann

- Die in den Bildern 4.25 und 4.26 dargestellten Fälle sind dadurch festgelegt, daß die Dikke der Unterkonstruktion so klein ist, daß im Muttergewinde im Schnitt $r=d_{g} / 2$ die maximal mögliche Gewindehöhe $h_{\max }=P-c$ nicht erreicht wird $\left(\varphi_{\mathrm{t}}<2 \pi-\varphi_{c}\right)$. In diesen Fällen kann die Gewindehöhe des Muttergewindes im Schnitt $r=d_{g} / 2$ bis $z u$ der Stelle $\varphi=\varphi_{t}$ durch 
$\mathrm{h}=\mathrm{Z}=\varphi^{*} \mathrm{P} /\left(2^{*} \pi\right)$ beschrieben werden und an der Stelle $\varphi=\varphi_{\mathrm{t}}$ ist die für diese Fälle maximal mögliche Gewindehöhe $h_{\max , I}=t$ erreicht. Diese Höhe wird im Auslauf des Muttergewindes $\varphi_{\mathrm{t}} \leq \varphi \leq \varphi_{\mathrm{t}}+\varphi_{\text {EA }}$ im Schnitt $\mathrm{r}=\mathrm{d}_{\mathrm{g}} / 2$ nur dann reduziert, wenn in diesem Bereich die Blechdikke der Unterkonstruktion durch einen auf der Oberkante der Unterkonstruktion einlaufenden Gewindegang der Schraube reduziert wird (die Bedingung hierfür lautet $\varphi_{\mathrm{t}}+\varphi_{\mathrm{EA}}>2 \pi-$ $\varphi_{c}$, vgl. auch Bild 4.28). Dieser Fall ist im Bild 4.25 dargestellt. In diesem Fall ist die reduzierte Höhe im Schnitt $r=d_{g} / 2$ auf dem Bereich $2 \pi-\varphi_{c}<\varphi \leq \varphi_{t}+\varphi_{\text {EA }}$ durch $h_{\text {red }}=t-(\varphi-(2 \pi-$ $\left.\left.\varphi_{c}\right)\right)^{*} \mathrm{P} /\left(2^{*} \pi\right)$ gegeben. Die Abscherfläche $A_{S, M G}$ für den im Bild 4.25 dargestellten Fall $2 \pi-$ $\varphi_{\mathrm{C}}-\varphi_{\mathrm{EA}}<\varphi_{\mathrm{t}}<2 \pi-\varphi_{\mathrm{c}}$ ergibt sich daher aus der Beziehung (4.8).

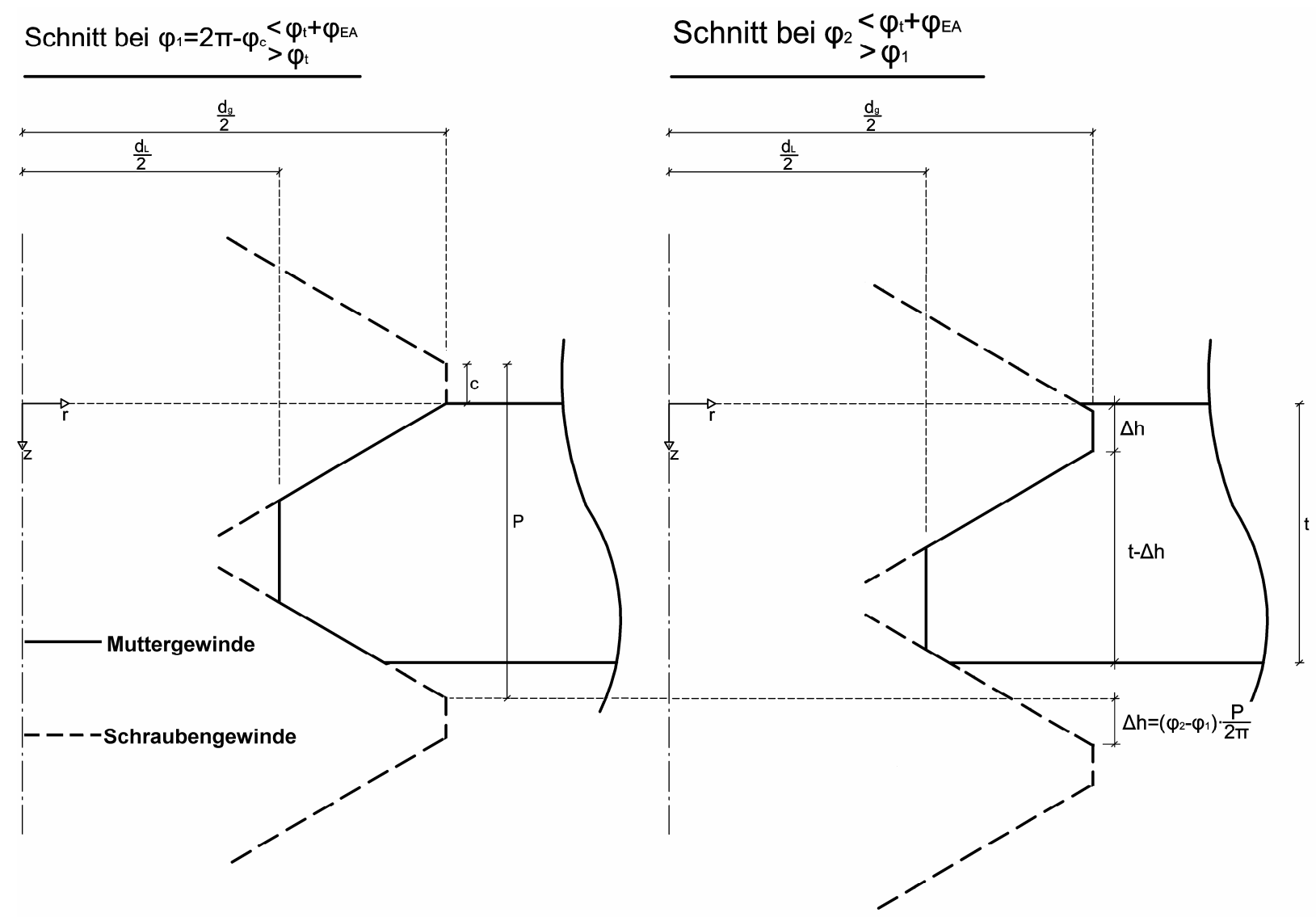

Bild 4.28: Reduktion der Gewindehöhe $h_{\text {max }, I I}=t$ für den Fall $\varphi_{t}<2 \pi-\varphi_{c}$ und $\varphi_{t}+\varphi_{E A}>2 \pi-\varphi_{c}$

- Der im Bild 4.26 dargestellte Fall $\varphi_{\mathrm{t}} \leq 2 \pi-\varphi_{\mathrm{c}}-\varphi_{\mathrm{EA}}$ unterscheidet sich gegenüber dem Fall $2 \pi-\varphi_{c}-\varphi_{E A}<\varphi_{t}<2 \pi-\varphi_{c}$ (Bild 4.25) nur dadurch, daß die Höhe $h_{\text {max, II }}=t$ im Schnitt $r=d_{g} / 2$ auf dem gesamten Bereich des Auslaufs des Muttergewindes $\varphi_{\mathrm{t}} \leq \varphi \leq \varphi_{\mathrm{t}}+\varphi_{\mathrm{EA}}$ erhalten bleibt. Die Abscherfläche $A_{S, M G}$ für diesen Fall $\varphi_{\mathrm{t}} \leq 2 \pi-\varphi_{\mathrm{c}}-\varphi_{\mathrm{EA}}$ ergibt sich daher aus der Beziehung (4.9). 


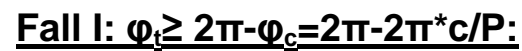

$$
\begin{aligned}
& \mathbf{A}_{\mathrm{S}, \mathrm{MG}}=\int_{0}^{2 \pi-\varphi_{\mathrm{c}}}\left(\varphi \cdot \frac{\mathbf{P}}{2 \pi} \cdot \frac{\mathbf{d}_{\mathbf{g}}}{2}\right) \mathbf{d} \varphi+\int_{0}^{\varphi_{\mathrm{t}}-2 \pi+\varphi_{\mathrm{c}}}\left[(\mathbf{P}-\mathbf{c}) \cdot \frac{\mathbf{d}_{\mathbf{g}}}{2}\right] \mathbf{d} \varphi+\int_{0}^{\varphi_{E A}}\left[\left(\mathbf{P}-\mathbf{c}-\varphi \cdot \frac{\mathbf{P}}{2 \pi}\right) \cdot \frac{\mathbf{d}_{\mathbf{g}}}{2}\right] \mathbf{d} \varphi \\
& =\frac{\mathbf{d}_{\mathbf{g}}}{2}\left[\left(2 \pi-\varphi_{\mathrm{c}}\right)^{2} \frac{\mathbf{P}}{4 \pi}+(\mathbf{P}-\mathbf{c}) \cdot\left(\varphi_{\mathbf{t}}-2 \pi+\varphi_{\mathrm{C}}\right)+\varphi_{\mathrm{EA}} \cdot\left(\mathbf{P}-\mathbf{c}-\frac{\mathbf{P}}{4 \pi} \varphi_{E A}\right)\right]
\end{aligned}
$$

\section{Fall II: $2 \pi-\varphi_{\underline{c}}-\underline{\varphi}_{E A} \leq \varphi_{t} \leq 2 \pi-\varphi_{c}:$}

$$
\begin{aligned}
& \mathrm{A}_{\mathrm{S}, \mathrm{MG}}=\int_{0}^{\varphi_{\mathrm{t}}}\left(\varphi \cdot \frac{P}{2 \pi} \cdot \frac{d_{g}}{2}\right) d \varphi+\int_{0}^{2 \pi-\varphi_{\mathrm{c}}-\varphi_{t}}\left[t \cdot \frac{d_{g}}{2}\right] d \varphi+\int_{0}^{\varphi_{t}+\varphi_{E A}-2 \pi+\varphi_{c}}\left[\left(t-\varphi \cdot \frac{P}{2 \pi}\right) \cdot \frac{d_{g}}{2}\right] d \varphi \\
& =\frac{\mathbf{d}_{\mathbf{g}}}{2}\left[\varphi_{\mathrm{t}}{ }^{2} \frac{\mathbf{P}}{4 \pi}+\mathbf{t}\left(2 \pi-\varphi_{\mathrm{C}}-\varphi_{\mathbf{t}}\right)+\mathbf{t}\left(\varphi_{\mathrm{t}}+\varphi_{\mathrm{EA}}-2 \pi+\varphi_{\mathrm{C}}\right)-\frac{\mathbf{P}}{4 \pi}\left(\varphi_{\mathbf{t}}+\varphi_{\mathrm{EA}}-2 \pi+\varphi_{\mathrm{C}}\right)^{2}\right] \\
& =\frac{\mathbf{d}_{\mathbf{g}}}{2}\left[\varphi_{\mathbf{t}}{ }^{2} \frac{\mathbf{P}}{4 \pi}+\mathbf{t} \cdot \varphi_{\mathrm{EA}}-\frac{\mathbf{P}}{4 \pi}\left(\varphi_{\mathbf{t}}+\varphi_{\mathrm{EA}}-2 \pi+\varphi_{\mathrm{C}}\right)^{2}\right]
\end{aligned}
$$

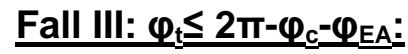

$$
\begin{aligned}
& \mathbf{A}_{\mathrm{S}, \mathrm{MG}}=\int_{0}^{\varphi_{\mathrm{t}}}\left(\varphi \cdot \frac{\mathbf{P}}{2 \pi} \cdot \frac{\mathbf{d}_{\mathbf{g}}}{2}\right) \mathbf{d} \varphi+\int_{0}^{\varphi_{\mathrm{EA}}}\left[\mathbf{t} \cdot \frac{\mathbf{d}_{\mathbf{g}}}{2}\right] \mathbf{d} \varphi \\
& =\frac{\mathbf{d}_{\mathbf{g}}}{2}\left[\varphi_{\mathrm{t}}{ }^{2} \frac{\mathbf{P}}{4 \pi}+\mathbf{t} \cdot \varphi_{\mathrm{EA}}\right]
\end{aligned}
$$

Mit der Fläche $A_{S, M G}$ und der Scherfestigkeit $\tau_{m, M G}$ der Gewindegänge des Muttergewindes ergibt sich für die Grenzsituation, daß der Mutterkörper nicht radial aufgeweitet wird, eine Abschätzung für die Abschertragfähigkeit des Muttergewindes $\mathrm{F}_{\mathrm{As}, \mathrm{MG}}^{*}$ aus:

$$
\mathrm{F}_{\mathrm{As}, \mathrm{MG}}^{*}=\tau_{\mathrm{m}, \mathrm{MG}}{ }^{*} \mathrm{~A}_{\mathrm{S}, \mathrm{MG}} \quad \text { mit: } \mathrm{A}_{\mathrm{S}, \mathrm{MG}} \text { gemäß (4.7) bis (4.9) }
$$

Die Kalibrierung der Beziehung (4.10) sowie die Berücksichtigung des tragfähigkeitsmindernden Einflusses, der aus einer radialen Aufweitung des Mutterkörpers zu erwarten ist, erfolgte anhand der Ergebnisse der Parameterstudien. Für die Scherfestigkeit $\tau_{\mathrm{m}, \mathrm{MG}}$ wurden dabei in die Beziehung (4.10) aufgrund der von Mises Hypothese, über die in den FE-Berechnungen die Vergleichsspannungen berechnet werden, die mit $\frac{1}{\sqrt{3}}=0,577$ multiplizierten Werte der wahren Zugfestigkeiten der für die Unterkonstruktionen verwendeten Werkstoffgesetzte eingesetzt $\left(\tau_{\mathrm{m}, \mathrm{MG}, \mathrm{S} 355}=362 \mathrm{~N} / \mathrm{mm}^{2}, \tau_{\mathrm{m}, \mathrm{MG}, \mathrm{S} 280}=280 \mathrm{~N} / \mathrm{mm}^{2}, \tau_{\mathrm{m}, \mathrm{MG}, \mathrm{Al}}=146 \mathrm{~N} / \mathrm{mm}^{2}\right)$. 


\section{Kombiniertes Versagen von Schrauben- und Muttergewinde:}

Dieser Versagensmechanismus ist dadurch bestimmt, daß sich im Grenzzustand des Versagens sowohl in den Gewindegängen des Schraubengewindes als auch in den Gewindegängen des Muttergewindes plastische Verformungen einstellen, die dazu führen, daß sich die Vertikalkomponenten der Kontaktspannungen zwischen dem Schraubengewinde und dem Muttergewinde hin zu einem Radius verlagern, der zwischen dem Durchmesser $d_{L}$ des Kernloches in der Unterkonstruktion und dem Außendurchmesser $d_{g}$ der Schraube liegt. Diese Verlagerung der Kontaktspannungen hin zu einem Schnitt $d_{L} / 2<r<d_{g} / 2$ ist folgendermaßen zu erklären:

- Sofern die Abschertragfähigkeit des Muttergewindes im Schnitt $r=d_{g} / 2$ gegenüber der Abschertragfähigkeit des Schraubengewindes im Schnitt $r=d_{\llcorner} / 2$ sehr groß ist, ergeben sich im Grenzzustand des Versagens keine plastischen Verformungen im Muttergewinde und die Auszugstragfähigkeit bestimmt sich aus dem oben beschriebenen Versagen des Schraubengewindes durch ein Abscheren der Gewindegänge der Schraube im Schnitt $r=d_{L} / 2$. Für den Fall einer Annäherung der Abschertragfähigkeit des Schraubengewindes im Schnitt $r=d_{\llcorner} / 2$ an die Abschertragfähigkeit des Muttergewindes im Schnitt $r=d_{g} / 2$ entstehen im Grenzzustand des Versagens auch im Muttergewinde plastische Verformungen, die dazu führen, daß sich die Vertikalkomponenten der Kontaktspannungen zwischen dem Schraubengewinde und dem Muttergewinde vom Schnitt $r=d_{\llcorner} / 2$ hin zu einem Schnitt verlagern, der im Verzahnungsbereich mit dem Muttergewinde weiter außen liegt $\left(r>d_{\llcorner} / 2\right)$. Je näher sich die Abschertragfähigkeit des Schraubengewindes im Schnitt $r=d_{\llcorner} / 2$ an die Abschertragfähigkeit des Muttergewindes im Schnitt $r=d_{g} / 2$ annähert, desto weiter verlagern sich die Kontaktspannungen hin zu dem Schnitt $r=d_{g} / 2$. Da die Abschertragfähigkeit in den Gewindegängen der Schraube in Schnitten mit größer werdendem Radius kleiner wird, ergibt sich die Auszugstragfähigkeit für den Fall, daß die Tragfähigkeit des Schraubengewindes gegenüber der Tragfähigkeit des Muttergewindes so groß ist, daß sich auch im Muttergewinde bereits plastische Verformungen einstellen, aus einer Abminderung der Abschertragfähigkeit des Schraubengewindes im Schnitt $r=d_{L} / 2$. Diese Abminderung ist umso größer je näher die Abschertragfähigkeit des Schraubengewindes im Schnitt $r=d_{\llcorner} / 2$ an der Abschertragfähigkeit des Muttergewindes im Schnitt $r=d_{g} / 2$ liegt.

- Für den umgekehrten Fall, daß die Abschertragfähigkeit des Muttergewindes im Schnitt $r=d_{g} / 2$ kleiner ist als die Abschertragfähigkeit des Schraubengewindes im Schnitt $r=d_{\llcorner} / 2$, verlagern sich für den Fall einer Annäherung dieser beiden Tragfähigkeiten die Vertikalkomponenten der Kontaktspannungen zwischen dem Schraubengewinde und dem Muttergewinde im Grenzzustand des Versagens aufgrund von sich ausbildenden plastischen Verformungen im Schraubengewinde vom Schnitt $r=d_{g} / 2$ hin zu einem Schnitt, der im Verzahnungsbereich mit dem Muttergewinde weiter innen liegt $\left(r<d_{g} / 2\right)$. Je näher sich die 
Abschertragfähigkeit des Muttergewindes im Schnitt $r=d_{g} / 2$ an die Abschertragfähigkeit des Schraubengewindes im Schnitt $r=d_{\llcorner} / 2$ annähert, desto weiter verlagern sich die Kontaktspannungen hin zu dem Schnitt $r=d_{\llcorner} / 2$. Da die Abschertragfähigkeit in den Gewindegängen des Muttergewindes in Schnitten mit kleiner werdendem Radius kleiner wird, ergibt sich die Auszugstragfähigkeit für den Fall, daß die Tragfähigkeit des Muttergewindes gegenüber der Tragfähigkeit des Schraubengewindes so groß ist, daß sich auch im Schraubengewinde bereits plastische Verformungen einstellen, aus einer Abminderung der Abschertragfähigkeit des Muttergewindes im Schnitt $r=d_{g} / 2$. Diese Abminderung ist umso größer je näher die Abschertragfähigkeit des Muttergewindes im Schnitt $r=d_{g} / 2$ an der Abschertragfähigkeit des Schraubengewindes im Schnitt $r=d_{L} / 2$ liegt.

Aufgrund dieser Zusammenhänge erfolgte die Bestimmung der Auszugstragfähigkeit, die sowohl vom Schraubengewinde als auch vom Muttergewinde beeinflußt wird, durch eine Abminderung des kleineren der beiden Tragfähigkeitswerte, die aus dem Versagen des Schraubengewindes und dem Versagen des Muttergewindes resultieren. Aufgrund der obigen Erläuterungen kann die Abminderung durch eine Funktion beschrieben werden, die abhängig vom Verhältnis $V_{\mathrm{MG}, \mathrm{SG}}=\mathrm{F}_{\mathrm{As}, \mathrm{MG}} / \mathrm{F}_{\mathrm{As}, \mathrm{SG}}$ ist, das sich aus dem Quotient der beiden Tragfähigkeitswerte für das Versagen des Schraubengewindes und das Versagen des Muttergewindes ergibt. Für Verhältniswerte $\mathrm{V}_{\mathrm{MG}, \mathrm{SG}}=1 \mathrm{muß}$ die Abminderungsfunktion ein Maximum aufweisen und für Verhältniswerte $\mathrm{V}_{\mathrm{MG}, \mathrm{SG}} \neq 0$ oder $\mathrm{V}_{\mathrm{MG}, \mathrm{SG}} \neq \infty$ muß die Abminderungsfunktion gegen den Wert „1" streben. Die Festlegung der Abminderungsfunktion erfolgte mit Hilfe der Ergebnisse der Parameterstudien.

\subsubsection{Kalibrierung des mechanischen Modells anhand der Ergebnisse der Parame- terstudien}

Zur Kalibrierung des im Abschnitt 4.9.2.1 beschriebenen mechanischen Modells sowie zur Verdeutlichung, daß die unterschiedlichen Versagensmechanismen, die dem mechanischen Modell zugrunde liegen, tatsächlich für die Auszugstragfähigkeit maßgebend werden, wurden die FE-Berechnungen der Parameterstudien für die einzelnen Versagensmechanismen wie nachfolgend erläutert ausgewertet.

\section{Versagen des Schraubengewindes}

Zur Kalibrierung der durch die Beziehung (4.6) getroffenen Abschätzung für die Auszugstragfähigkeit, die ausschließlich durch ein Versagen des Schraubengewindes bestimmt ist, wurden die in den FE-Berechnungen erreichten Maximallasten $\mathrm{F}_{\mathrm{FE} \text {,max }}$ durch die aus der Beziehung (4.6) berechneten Werte $\mathrm{F}_{\mathrm{As}, \mathrm{SG}}^{*}$ dividiert. Die sich aus diesen Auswertungen ergebenden Verhältniswerte $\mathrm{F}_{\mathrm{FE}, \mathrm{max}} / \mathrm{F}_{\mathrm{As}, \mathrm{SG}}^{*}$ sind aus den Diagrammen D.13 bis D.24 des Anhangs $\mathrm{D}$ ersichtlich.

Aus den Auswertungen werden folgende Schlußfolgerungen gezogen: 
- Sofern die Beziehung (4.6) den Versagensfall des Abscherens der Gewindegänge der Schraube exakt beschreiben würde, müßten alle FE-Berechnungen, in denen dieser Versagensfall maßgebend wird, einen konstanten, oberen Grenzwert von $\mathrm{F}_{\mathrm{FE}, \max } / \mathrm{F}_{\mathrm{As}, \mathrm{SG}}^{\star}=1$ aufweisen. Da die Beziehung (4.6) als eine konservative Abschätzung angesehen werden kann, ist der Versagensfall des Abscherens der Gewindegänge der Schraube daran zu erkennen, daß alle FE-Berechnungen, in denen dieser Versagensfall maßgebend wird, einen konstanten, oberen Grenzwert $\mathrm{F}_{\mathrm{FE}, \mathrm{max}} / \mathrm{F}_{\mathrm{As}, \mathrm{SG}}^{*}>1$ ergeben.

- Dieser obere Grenzwert ist am deutlichsten anhand der Auswertungen für die Schrauben „A“, „C“ und „Cvc“ (Diagramme D.19 bis D.21) zu erkennen. Für diese Schrauben wird für den Versagensfall des Abscherens der Gewindegänge der Schraube ein mittlerer, oberer Grenzwert von $\left(\mathrm{F}_{\mathrm{FE}, \text { max }} / \mathrm{F}_{\mathrm{As}, \mathrm{SG} \text {,mittel }}^{*}\right)_{\text {mittel }}=1,20$ durch $22 \mathrm{FE}-$ Berechnungen bestätigt. Der in diesen Berechnungen erreichte maximale Wert beträgt $\left(\mathrm{F}_{\mathrm{FE}, \max } / \mathrm{F}_{\mathrm{As}, \mathrm{SG}}^{*}\right)_{\max }=1,26$ (FEBerechnung mit der Werkstoffpaarung „CH_S355“ und der Blechdicke 4,50mm für die Schraube „A“) und der minimale Wert beträgt $\left(\mathrm{F}_{\mathrm{FE}, \max } / \mathrm{F}_{\mathrm{As}, \mathrm{SG}}^{\star}\right)_{\min }=1,12$ (FE-Berechnung mit der Werkstoffpaarung „NRL_S280“ und der Blechdicke 4,90mm für die Schraube „C“).

- Der Verlauf der Verhältniswerte $F_{\mathrm{FE}, \max } / \mathrm{F}_{\mathrm{As}, \mathrm{SG}}^{*}$ für die $\mathrm{FE}-$ Serien mit unterschiedlichen Werkstoffpaarungen zeigt, daß die Verhältniswerte zunächst mit größer werdender Blechdicke zunehmen und dann - sofern der Versagensfall des Abscherens der Gewindegänge der Schraube maßgebend wird - ab einer bestimmten Grenzdicke $t_{\text {grenz }}$ der Unterkonstruktion konstant bleiben. Dieser Verlauf ist dadurch zu erklären, daß die Tragfähigkeit und die Steifigkeit des in der Unterkonstruktion geformten Muttergewindes mit größer werdender Blechdicke der Unterkonstruktion überproportional ansteigt, während die Tragfähigkeit des Schraubengewindes, die durch das Abscheren der Gewindegänge der Schraube bestimmt ist, gemäß (4.6) nur linear mit der Blechdicke zunimmt. Für Blechdicken $\mathrm{t}<\mathrm{t}_{\text {grenz }}$ werden daher die Versagensmechanismen, die von der Tragfähigkeit und der Steifigkeit des Muttergewindes beeinflußt werden, für die Auszugstragfähigkeit maßgebend, und für Blechdicken $\mathrm{t} \geq \mathrm{t}_{\text {grenz }}$ wird das Abscheren der Gewindegänge der Schrauben für die Auszugstragfähigkeit maßgebend. Weiterhin ist zu erkennen, daß die Grenzdicke $t_{\text {grenz }}$ kleiner wird, wenn der Quotient gebildet aus der Zugfestigkeit der Gewindegänge der Schraube und der Zugfestigkeit der Unterkonstruktion kleiner wird. Dies liegt darin begründet, daß für diesen Fall die Tragfähigkeit des Schraubengewindes gegenüber der Tragfähigkeit des Muttergewindes abnimmt und somit der Versagensfall des Abscherens der Gewindegänge der Schraube bereits bei kleineren Blechdicken maßgebend wird. Die kleinsten Dicken $t_{\text {grenz }}$ werden daher in den FE-Berechnungen mit der Werkstoffpaarung „NRL_S355“ erreicht.

- Für die Schrauben „F1“, „B“ und „D“ (Diagramme D.13, D.15 und D.22) tritt der Versagensfall des Abscherens der Gewindegänge der Schraube in den FE- 
Berechnungen nicht auf. Dies ist daran zu erkennen, daß die Verhältniswerte $\mathrm{F}_{\mathrm{FE}, \mathrm{max}} / \mathrm{F}_{\mathrm{As}, \mathrm{SG}}^{*}$ auch in den Berechnungen mit der Werkstoffpaarung „NRL_S355“ im gesamten Blechdickenbereich beim Übergang von einer Blechdicke $t_{1}$ auf eine größere Blechdicke $t_{2}$ noch deutlich ansteigen.

- Für die restlichen Schrauben „F2“, „G“, „J“, „H“, „E“ und „I“ (Diagramme D.14, D.16 bis D.18, D.23 und D.24) zeigt der Verlauf der Verhältniswerte $F_{F E, m a x} / F_{A s, S G}^{*}$, daß der Versagensfall des Abscherens der Gewindegänge der Schraube maximal in den FEBerechnungen mit der Werkstoffpaarung „NRL_S355“ und Blechdicken $\mathrm{t} \geq 3,94 \mathrm{~mm}$ gerade noch vorliegen kann.

Da der Versagensfall des Abscherens der Gewindegänge der Schrauben am deutlichsten in den FE-Berechnungen für die Schrauben „A“, „C“ und „Cvc“ nachgewiesen werden konnte, und da die entsprechenden FE-Berechnungen für diesen Versagensfall einen mittleren Ver-

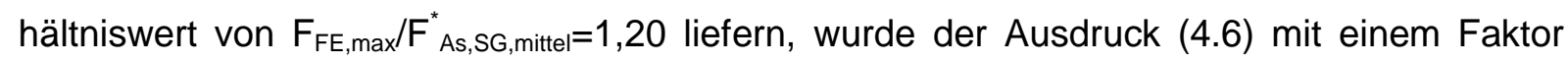
1,20 multipliziert. Damit ergibt sich die Auszugstragfähigkeit für den Versagensfall des Abscherens der Gewindegänge der Schraube aus:

$\mathrm{F}_{\mathrm{As}, \mathrm{SG}}=1,2^{*} T_{\mathrm{m}, \mathrm{SG}}{ }^{*}\left[\mathrm{C}+\left(\mathrm{d}_{\mathrm{g}}-\mathrm{d}_{\mathrm{L}}\right)^{*} \tan (\alpha / 2)\right]^{*} \mathrm{~d}_{\mathrm{L}}{ }^{*} \mathrm{t}^{*} \mathrm{~T} / \mathrm{P}$

\section{Versagen des Muttergewindes}

Die durch die Beziehung (4.10) getroffene Abschätzung beschreibt für die Grenzsituation, daß der Mutterkörper nicht radial aufgeweitet wird, den Versagensfall, der ausschließlich durch die Form und die Werkstoffestigkeit des Muttergewindes bestimmt wird. Zur Kalibrierung dieser Abschätzung sowie zur Berücksichtigung des tragfähigkeitsmindernden Einflusses einer radialen Aufweitung des Mutterkörpers wurden die Parameterstudien folgendermaßen ausgewertet:

- Für die Auswertungen wurden die FE-Berechnungen mit der Werkstoffpaarung „CH_Al“ herangezogen, da für diese Berechnungen der größte Quotient gebildet aus der wahren Zugfestigkeit der Gewindegänge der Schraube und der wahren Zugfestigkeit der Unterkonstruktion $R_{m, \text { wahr,CH}} / R_{m, \text { wahr,Al }}=1309 / 253=5,2$ aller FE-Berechnungen vorliegt. Da die Tragfähigkeit des Schraubengewindes gegenüber der Tragfähigkeit des Muttergewindes daher bei diesen Berechnungen am größten ist, werden die Versagensmechanismen, die ausschließlich durch das Muttergewinde bestimmt sind, am Besten aus diesen Berechnungen deutlich. Die in diesen Berechnungen für die unterschiedlichen Schrauben erreichten Maximallasten sind im Anhang D im Diagramm D.25 dargestellt.

- Im Anhang D im Diagramm D.26 wurden die aus der Beziehung (4.10) berechneten Werte $\mathrm{F}_{\mathrm{As}, \mathrm{MG}}^{\star}$ durch die in den FE-Berechnungen mit der Werkstoffpaarung „CH_Al“ erreichten Maximallasten $\mathrm{F}_{\mathrm{FE} \text {,max }}$ dividiert. 
Aus den Auswertungen werden folgende Sachverhalte deutlich:

- Das Diagramm D.25 zeigt, daß die in den FE-Berechnungen erreichten Maximallasten mit zunehmender Blechdicke überproportional ansteigen.

- Aus den Auswertungen im Diagramm D.26 wird deutlich, daß die in den FEBerechnungen erreichten Maximallasten für die kleinste untersuchte Blechdicke $\mathrm{t}=0,60 \mathrm{~mm}$ gut mit den berechneten Werten $\mathrm{F}_{\mathrm{As}, \mathrm{MG}}^{*}$ übereinstimmen. Für diese Blechdicke liegen die Werte $\mathrm{F}_{\mathrm{As}, \mathrm{MG}}^{*}$ nur maximal $12 \%$ über und $6 \%$ unter den in den FEBerechnungen erreichten Maximallasten.

- Mit ansteigender Blechdicke steigen die Verhältniswerte $F_{A s, M G}^{*} / F_{F E \text {,max }}$ zunächst an und erreichen dann bei einer Blechdicke im Bereich von ca. $1 \mathrm{~mm}$ bis $2 \mathrm{~mm}$ ihre Extremwerte. Die größten Verhältniswerte $F_{A s, M G}^{*} / F_{F E, m a x}$ ergeben sich für die Schrauben „A“ $\left(F^{*}{ }_{A s, M G} / F_{F E, \max }=1,51\right)$ und „J“ $\left(F^{*}{ }_{A s, M G} / F_{F E, \max }=1,36\right)$, die von allen untersuchten Schrauben die kleinsten Verzahnungen mit dem Muttergewinde $\left(d_{g}-d_{L}=0,30 \mathrm{~mm}\right.$ für die Schraube „A“ und $d_{g}-d_{L}=0,60 \mathrm{~mm}$ für die Schraube „J“) aufweisen. Nach dem Erreichen ihrer Extremwerte fallen die Verhältniswerte mit ansteigender Blechdicke wieder ab. Für die Schrauben mit den kleinsten Gewindeaußendurchmesser (Schrauben „D“, „E“, „“ mit einem Gewindeaußendurchmesser im Bereich von 4,64mm $\leq d_{g} \leq 4,76 \mathrm{~mm}$ und Schraube „H“ mit $\mathrm{d}_{\mathrm{g}}=5,32 \mathrm{~mm}$ ) sowie für die Schraube „F2“, die den größten Gewindeaußendurchmesser $\left(d_{g}=6,35 \mathrm{~mm}\right)$ aber auch gleichzeitig eine große Verzahnung mit der Unterkonstruktion aufweist $\left(d_{g}-d_{L}=1,35 m m\right)$, werden Verhältniswerte $F_{A s, M G}^{*} / F_{F E, m a x}<1$ erreicht. Die Blechdik$k e$, bei der für diese Schrauben der Verhältniswert $\mathrm{F}_{\mathrm{As}, \mathrm{MG}}^{\star} / \mathrm{F}_{\mathrm{FE}, \max }=1$ erreicht wird, ist um so kleiner je kleiner der Gewindeaußendurchmesser der Schrauben ist. Weiterhin ist diese Blechdicke für die Schrauben „D“, „E“ und „I“, die den selben Nennwert des Gewindeaußendurchmessers $d_{g, N}=4,8 \mathrm{~mm}$ besitzen, um so kleiner je größer ihre Verzahnung mit der Unterkonstruktion ist - also je kleiner der Kernlochdurchmesser $d_{L}$ in der Unterkonstruktion für diese Schrauben ist $\left(d_{L}=3,38 \mathrm{~mm}\right.$ für die Schraube „D“, $d_{L}=3,66 \mathrm{~mm}$ für die Schraube „E“ und $d_{L}=4,00 \mathrm{~mm}$ für die Schraube „I").

- Der beschriebene Verlauf der Verhältniswerte $F_{A s, M G}^{*} / F_{F E, m a x}$ ist durch den Einfluß der radialen Aufweitung des Muttergewindes zu erklären, durch den die Abschertragfähigkeit des Muttergewindes im Schnitt $r=d_{g} / 2$ vermindert wird. Für die kleinste untersuchte Blechdicke $\mathrm{t}=0,60 \mathrm{~mm}$ stellt sich nur eine geringe radiale Aufweitung des Mutterkörpers ein, da die zu übertragenden Kontaktkräfte zwischen dem Schrauben- und dem Muttergewinde bis zum Erreichen der Abschertragfähigkeit des Muttergewindes relativ klein bleiben. Mit größer werdender Blechdicke steigt die Abschertragfähigkeit des radial nicht aufgeweiteten Muttergewindes bis zu einer Blechdicke, die für die unterschiedlichen Schrauben in einem Bereich von $1 \mathrm{~mm}$ bis $2 \mathrm{~mm}$ liegt, nahezu quadratisch an, da sich für diesen Blech- 
dickenbereich die Abscherfläche des nicht radial aufgeweiteten Muttergewindes aufgrund des Abgrenzungskriteriums für den Fall III $\varphi_{\mathrm{t}} \leq 2 \pi-\varphi_{\mathrm{c}}-\varphi_{\mathrm{EA}} \hat{U}$ $\mathrm{t} \leq \mathrm{P}-\mathrm{c}-0,5^{\star}\left(\mathrm{d}_{\mathrm{g}}-\mathrm{d}_{\mathrm{L}}\right)^{\star} \tan (\alpha / 2)$ nach der Formel (4.9) berechnet, in die der Parameter $\varphi_{\mathrm{t}}=\mathrm{t}^{\star} 2 \pi / \mathrm{P}$ quadratisch eingeht. In diesem Blechdickenbereich steigen die Verhältniswerte $\mathrm{F}_{\mathrm{As}, \mathrm{MG}}^{*} / \mathrm{F}_{\mathrm{FE}, \max }$ an, da der durch die größer werdende Blechdicke erzielte Steifigkeitszuwachs im Mutterkörper nicht ausreicht, um die mit der Blechdicke quadratisch ansteigenden Abschertragfähigkeiten des radial nicht aufgeweiteten Muttergewindes zu erreichen. Für Blechdicken $t>P-c-0,5^{*}\left(d_{g}-d_{L}\right)^{*} \tan (\alpha / 2)$ steigen die Abschertragfähigkeiten des radial nicht aufgeweiteten Muttergewindes nur noch linear mit der Blechdicke an, weil dann die Formeln (4.7) und (4.8) für die Berechnung der Abscherflächen maßgebend werden. In diesem Blechdickenbereich fallen die Verhältniswerte $\mathrm{F}_{\mathrm{As}, \mathrm{MG}}^{*} / \mathrm{F}_{\mathrm{FE}, \max }$ wieder $a b$, weil der mit der steigenden Blechdicke erzielte Steifigkeitszuwachs im Mutterkörper zu einer Annäherung der Versagenslasten an die in diesem Blechdickenbereich nur noch linear mit der Blechdicke anwachsenden Abschertragfähigkeiten des radial nicht aufgeweiteten Muttergewindes führt. Der für die Schrauben „D“, „E“, „l“, „H“ und „F2“ beobachtete Sachverhalt, daß die Blechdicke, bei der ein Verhältniswerte $F_{A s, M G}^{*} / F_{F E, m a x}=1$ erreicht wird, um so kleiner ist je kleiner der Gewindeaußendurchmesser $d_{g}$ der Schraube und je größer die Verzahnung $d_{g}-d_{L}$ der Schraube mit der Unterkonstruktion ist, kann ebenfalls über die radiale Aufweitung des Muttergewindes erklärt werden. Mit kleiner werdendem Gewindeaußendurchmesser steigt die Steifigkeit des Muttergewindes gegen eine radiale Aufweitung an, weil die Krümmung des Muttergewindes im Schnitt $r=d_{g} / 2$ größer wird. Der tragfähigkeitsmindernde Einfluß der radialen Mutteraufweitung ist daher für Schrauben mit einem kleinen Gewindeaußendurchmesser weniger stark ausgeprägt als bei Schrauben mit einem größeren Gewindeaußendurchmesser. Der ungünstige Einfluß einer kleinen Verzahnung der Schraube mit der Unterkonstruktion wurde bereits im Abschnitt 4.9.2.1 dadurch erklärt, daß eine geringe Verzahnung zusammen mit der radialen Aufweitung des Muttergewindes dazu führt, daß im oberen Bereich der Unterkonstruktion (Biegezugbereich) die Verzahnung zwischen Schrauben- und Muttergewinde verloren geht, und somit in diesem Bereich die Gewindegänge des Muttergewindes nicht abgeschert werden müssen. Dieser ungünstige Einfluß einer geringen Verzahnung ist besonders deutlich aus den Auswertungen im Diagramm D.26 für die Schrauben „A“ und „J“ zu erkennen.

- Die im Diagramm D.26 auftretenden Verhältniswerte $F_{A s, M G}^{*} / F_{F E, m a x}<1$ sind dadurch $z u$ erklären, daß die Beziehung (4.10) für den Fall, daß sich keine radiale Aufweitung des Muttergewindes ausbildet, eine konservative Abschätzung für die Abschertragfähigkeit des Muttergewindes liefert, weil sich - analog zu den Verhältnissen für das Schraubengewinde -aufgrund der Lastausbreitung auch Bereiche im Muttergewinde an der Lastabtragung beteiligen, die vor allem in radialer Richtung aber auch in zirkularer Richtung ü- 
ber den durch die Fläche $A_{S, M G}$ abgedeckten Bereich hinausgehen können. Für große Blechdicken sowie für Schrauben mit einem kleinen Gewindeaußendurchmesser und einer großen Verzahnung in der Unterkonstruktion - diese Randbedingungen führen aufgrund der obigen Erläuterungen zu einer geringen radialen Aufweitung des Muttergewindes - werden daher Verhältniswerte $\mathrm{F}_{\mathrm{As}, \mathrm{MG}}^{*} / \mathrm{F}_{\mathrm{FE}, \max }<1$ erreicht.

Zur formelmäßigen Beschreibung der Auszugstragfähigkeit, die ausschließlich durch die Form und die Werkstoffestigkeit des Muttergewindes bestimmt wird, wurde den oben beschriebenen Sachverhalten folgendermaßen Rechnung getragen:

- Der mit der Blechdicke t überproportional ansteigende Verlauf der Auszugstragfähigkeit wurde durch eine Potenzfunktion angenähert:

$\mathrm{F}_{\mathrm{As}, \mathrm{MG}}=\mathrm{a} \cdot \mathrm{t}^{\mathrm{b}}$

- Zur Bestimmung der Parameter a und b dieser Potenzfunktion wurde die durch die Beziehung (4.10) getroffene Abschätzung der Auszugstragfähigkeit für die Grenzsituation, daß der Mutterkörper nicht radial aufgeweitet ist, an zwei Stützstellen (Blechdicken $t_{1}$ und $t_{2}$ ) ausgewertet, an denen der Effekt der radialen Aufweitung des Muttergewindes aufgrund der Auswertungen im Diagramm D.26 abgeschätzt werden kann.

- Für die untere Stützstelle wurde die Blechdicke $t_{1}=0,60 \mathrm{~mm}$ herangezogen. Für diese Blechdicke stimmen die aus der Beziehung (4.10) berechneten Werte $F_{\text {As,MG }}^{*}$ gut mit den FE-Ergebnissen überein und liegen im Mittel nur 5\% über den in den FE-Berechnungen erreichten Maximallasten. Für die Stützstelle $t_{1}=0,60 \mathrm{~mm}$ wurde daher für die Auszugstragfähigkeit die nachfolgend angegebene Beziehung (4.13) zugrunde gelegt. Da für alle untersuchten Schrauben an der Stützstelle $t_{1}=0,60 \mathrm{~mm}$ die Bedingung $\varphi_{\mathrm{t}} \leq 2 \pi-\varphi_{\mathrm{c}}-\varphi_{\text {EA }}$ erfüllt ist, ergeben sich die zur Berechnung von $\mathrm{F}_{\mathrm{As}, \mathrm{MG}}^{*}\left(\mathrm{t}=\mathrm{t}_{1}\right)$ benötigten Abscherflächen aus der Beziehung (4.9).

$\mathrm{F}_{\mathrm{As}, \mathrm{MG}}\left(\mathrm{t}=\mathrm{t}_{1}\right)=\mathrm{a} \cdot \mathrm{t}_{1}{ }^{\mathrm{b}}=0,95 \cdot \mathrm{F}^{*}{ }_{\mathrm{As}, \mathrm{MG}}\left(\mathrm{t}=\mathrm{t}_{1}\right)=0,95 \cdot \tau_{\mathrm{m}, \mathrm{MG}} \cdot \frac{\mathrm{d}_{\mathrm{g}}}{2}\left[\varphi_{\mathrm{t}_{1}}{ }^{2} \frac{\mathrm{P}}{4 \pi}+\mathrm{t}_{1} \cdot \varphi_{\mathrm{EA}}\right]$

- Die Blechdicke $t_{2}$ für die obere Stützstelle wurde mit Hilfe der Ergebnisse im Diagramm D.26 so abgeschätzt, daß sie in etwa diejenige Blechdicke beschreibt, an der die Verhältniswerte $\mathrm{F}_{\mathrm{As}, \mathrm{MG}}^{*} / \mathrm{F}_{\mathrm{FE} \text {,max }}$ nach dem Erreichen ihrer Extremwerte auf den Wert „" “ zurückgehen. Diese Blechdicke ist aufgrund der obigen Ausführungen vom Gewindeaußendurchmesser $d_{g}$ der Schraube sowie von der Verzahnung der Schraube in der Unterkonstruktion $d_{g}-d_{L}$ abhängig. Die Blechdicke $t_{2}$ muß um so größer werden je größer der Gewindeaußendurchmesser der Schraube und je kleiner die Verzahnung der Schraube mit der Unterkonstruktion ist. Da mit größer werdendem Gewindeaußendurchmesser und gleichbleibendem Lochdurchmesser die Verzahnung in der Unterkonstruktion zunimmt, besitzt 
die Größe des Gewindeaußendurchmessers zwei gegenläufige Wirkungen auf die Größe der Blechdicke $t_{2}$. Aus diesem Grund läßt sich die Blechdicke $t_{2}$ gut durch die Beziehung (4.14) beschreiben, die nur noch vom Lochdurchmesser $d_{L}$ abhängig ist.

$$
t_{2}= \begin{cases}0,9 \cdot d_{L} & \text { für } d_{L} \leq 4 m m \\ d_{L} & \text { für } 4 m m<d_{L} \leq 5 m m \\ 1,1 \cdot d_{L} & \text { für } d_{L}>5 m m\end{cases}
$$

- Mit Hilfe der Beziehung (4.14) ergibt sich die Auszugstragfähigkeit für die Stützstelle $\mathrm{t}_{2}$ aus der Beziehung (4.15). Da für alle untersuchten Schrauben an der Stützstelle $t_{2}$ die Bedingung $\varphi_{t} \geq 2 \pi-\varphi_{c}$ erfüllt ist, ergeben sich die zur Berechnung von $F_{A s, M G}^{*}\left(t=t_{2}\right)$ benötigten Abscherflächen aus der Beziehung (4.7).

$$
\begin{aligned}
& \mathrm{F}_{\mathrm{As}, \mathrm{MG}}\left(\mathrm{t}=\mathrm{t}_{2}\right)=\mathrm{a} \cdot \mathrm{t}_{2}{ }^{\mathrm{b}}=\mathrm{F}^{*}{ }_{\mathrm{As}, \mathrm{MG}}\left(\mathrm{t}=\mathrm{t}_{2}\right) \\
& =\tau_{\mathrm{m}, \mathrm{MG}} \cdot \frac{\mathrm{d}_{\mathrm{g}}}{2}\left[\left(2 \pi-\varphi_{\mathrm{c}}\right)^{2} \frac{\mathrm{P}}{4 \pi}+(\mathrm{P}-\mathrm{c}) \cdot\left(\varphi_{\mathrm{t}_{2}}-2 \pi+\varphi_{\mathrm{c}}\right)+\varphi_{\mathrm{EA}} \cdot\left(\mathrm{P}-\mathrm{c}-\frac{\mathrm{P}}{4 \pi} \varphi_{\mathrm{EA}}\right)\right]
\end{aligned}
$$

- Mit Hilfe der Beziehungen (4.13) bis (4.15) erhält man die zur Beschreibung der Auszugstragfähigkeit für die Beziehung (4.12) benötigten Parameter a und b aus den Gleichungen (4.16) und (4.17).

$$
\begin{aligned}
& \mathrm{a}=\frac{0,95 \cdot \mathrm{F}^{*}{ }_{\mathrm{As}, \mathrm{MG}}\left(\mathrm{t}=\mathrm{t}_{1}\right)}{\mathrm{t}_{1}{ }^{\mathrm{b}}} \\
& \mathrm{b}=\frac{\ln \left(\frac{\mathrm{F}^{*}{ }_{\mathrm{As}, \mathrm{MG}}\left(\mathrm{t}=\mathrm{t}_{2}\right)}{0,95 \cdot \mathrm{F}^{*}{ }_{\mathrm{As}, \mathrm{MG}}\left(\mathrm{t}=\mathrm{t}_{1}\right)}\right)}{\ln \left(\frac{\mathrm{t}_{2}}{\mathrm{t}_{1}}\right)}
\end{aligned}
$$

Im Anhang D im Diagramm D.27 sind für die FE-Berechnungen mit der Werkstoffpaarung „CH_Al“ die durch die Beziehung (4.12) abgeschätzten Auszugstragfähigkeiten $\mathrm{F}_{\mathrm{As}, \mathrm{MG}}$ über den in den FE-Berechnungen erreichten Maximallasten $F_{F E \text {,max }}$ aufgetragen. Wie aus diesem Diagramm ersichtlich ist, werden durch die Beziehung (4.12) die in den FE-Berechnungen erreichten Maximallasten gut angenähert. Die abgeschätzten Auszugstragfähigkeiten $\mathrm{F}_{\mathrm{As}, \mathrm{MG}}$ liegen maximal 11\% über (Berechnung für die Schraube „A“ und die Blechdicke 1,99mm) und $11 \%$ unter (Berechnung für die Schraube „G“ und die Blechdicke 0,60mm) den Maximallasten $\mathrm{F}_{\mathrm{FE} \text {,max }}$ der $\mathrm{FE}-$ Berechnungen.

\section{Kombiniertes Versagen von Schrauben- und Muttergewinde:}

Zur Bestimmung der Funktion, mit der der kleinere der beiden Tragfähigkeitswerte aus dem Versagen des Schraubengewindes $\left(F_{\mathrm{As}, \mathrm{SG}}\right.$ nach Formel (4.11)) und dem Versagen des Muttergewindes $\left(F_{A s, M G}\right.$ nach Formel (4.12)) abgemindert werden muß für den Fall, daß diese 
beiden Tragfähigkeiten so nahe beieinander liegen, daß sich im Grenzzustand des Versagens sowohl im Schraubengewinde als auch im Muttergewinde plastische Verformungen einstellen, wurden die FE-Berechnungen der Parameterstudien folgendermaßen ausgewertet:

- Der Quotient [Min $\left.\left(\mathrm{F}_{\mathrm{As}, \mathrm{SG}}, \mathrm{F}_{\mathrm{As}, \mathrm{MG}}\right)\right] / \mathrm{F}_{\mathrm{FE} \text {,max }}$ gebildet aus dem kleineren der beiden nach den Formeln (4.11) und (4.12) berechneten Tragfähigkeiten und den in den FEBerechnungen erreichten Maximallasten wurde über das Verhältnis $V_{M G, S G}=F_{A s, M G} / F_{A s, S G}$ der berechneten Tragfähigkeitswerte aufgetragen.

- Diese Auswertungen sind für die in den Parameterstudien untersuchten unterschiedlichen Werkstoffpaarungen für das Schraubengewinde und für die Unterkonstruktion in den Diagrammen D.28 bis D.33 des Anhangs D dargestellt.

- Der Verlauf der gesuchten Abminderungsfunktion wird mit Hilfe dieser Auswertungen durch den Verlauf der Quotienten $\left[\mathrm{Min}\left(\mathrm{F}_{\mathrm{As}, \mathrm{SG}}, \mathrm{F}_{\mathrm{As}, \mathrm{MG}}\right)\right] / \mathrm{F}_{\mathrm{FE}, \max }>1$ beschrieben.

- Als eine obere Annäherung an diesen Verlauf wurde die in der Beziehung (4.18) angegebenen Abminderungsfunktion $\mathrm{R}\left(\mathrm{V}_{\mathrm{MG}, \mathrm{SG}}\right)$ verwendet. Der Verlauf dieser Abminderungsfunktion ist ebenfalls in den Diagrammen D.28 bis D.33 dargestellt.

$$
R\left(V_{M G, S G}\right)= \begin{cases}1+0,30 \cdot \mathrm{e}^{\left(1,95 \cdot\left(1-\frac{1}{V_{M G, S G}}\right)\right)} & \text { für } V_{M G, S G} \leq 1 \\ 1+0,30 \cdot \mathrm{e}^{\left(2,5 \cdot\left(1-V_{M G}, S G\right)\right.} & \text { für } V_{M G, S G} \geq 1\end{cases}
$$

Mit Hilfe der Beziehungen (4.11) bis (4.18) ergibt sich die formelmäßige Beschreibung der Auszugstragfähigkeit, die den drei im Abschnitt 4.9.2.1 beschriebenen Versagensmechanismen Rechnung trägt, aus der nachfolgend angegebenen Beziehung (4.19).

$$
\mathrm{F}_{\mathrm{AZV}}=\frac{\operatorname{Minimum}\left(\mathrm{F}_{\mathrm{As}, \mathrm{SG}}, \mathrm{F}_{\mathrm{As}, \mathrm{MG}}\right)}{\mathrm{R}\left(\mathrm{V}_{\mathrm{MG}, \mathrm{SG}}\right)}
$$

Im Anhang D in den Diagrammen D.34 bis D.39 sind für die in den Parameterstudien untersuchten unterschiedlichen Werkstoffpaarungen die nach (4.11) bis (4.19) berechneten Auszugstragfähigkeiten $\mathrm{F}_{\mathrm{AZV}}$ über den in den FE-Berechnungen erreichten Maximallasten $\mathrm{F}_{\mathrm{FE} \text {,max }}$ aufgetragen. Wie aus diesen Diagrammen ersichtlich ist, werden die in den FEBerechnungen erreichten Maximallasten gut durch die berechneten Werte angenähert. Die berechneten Auszugstragfähigkeiten $F_{A Z v}$ liegen im Mittel 3\% unter und maximal 13\% über (FE-Berechnung mit der Werkstoffpaarung „NRL_Al“ und der Blechdicke 3,06mm für die Schraube „J") und 12\% unter (FE-Berechnung mit der Werkstoffpaarung „CH_S280“ und der Blechdicke 0,69mm für die Schraube "Cvc“) den Maximallasten $F_{\mathrm{FE}, \max }$ der FEBerechnungen. 


\section{$5 \quad$ Entwicklung eines Bemessungsmodells}

\subsection{Anwendung des mechanischen Modells auf die Versuchsergebnisse}

Der erste Schritt zur Entwicklung eines Bemessungsmodells ist die Anwendung des im Abschnitt 4.9.2 beschriebenen mechanischen Modells auf die in der Versuchsdatenbank (Anhang A, Tabellen A.1 und A.2) aufgeführten Versuchsergebnisse. Dieser Schritt wird dadurch vollzogen, daß mit Hilfe des mechanischen Modells für jede der 230 unterschiedlichen Versuchsserien der Versuchsdatenbank ein mittlerer Tragfähigkeitswert $r_{t, \text { mittel }}$ berechnet wird, der mit den in den Versuchsserien erreichten mittleren Versagenslasten $r_{v, \text { mittel }}$ (Tabelle A.1, Spalte 12) verglichen wird. Die berechnete Tragfähigkeit ist selbst dann, wenn das mechanische Modell die Wirklichkeit genau beschreiben würde, lediglich als eine Abschätzung für die mittlere Versagenslast einer Versuchsserie anzusehen, da die für die Anwendung des mechanischen Modells in den Beziehungen (4.11) bis (4.19) benötigten Einflußgrößen einerseits Streuungen unterliegen, aber auch weil andererseits zur Festlegung von repräsentativen Mittelwerten für diese Einflußgrößen in der Versuchsdatenbank

- für die Gewindeabflachung c sowie in den Versuchen, die mit Bohrschrauben durchgeführt wurden, auch für den Lochdurchmesser $d_{\llcorner}$in der Unterkonstruktion keine Informationen vorliegen,

- $\quad$ teilweise keine Meßwerte für die Mittelwerte der Einflußgrößen dokumentiert sind und

- sofern Meßwerte vorliegen, teilweise nicht bekannt ist, ob diese Meßwerte an Proben ermittelt wurden, die aus der gleichen Charge stammen, wie die für die Auszugversuche verwendeten Proben.

Die Güte des Vergleichs zwischen den in den Versuchsserien erreichten mittleren Versagenslasten und den berechneten Tragfähigkeitswerten ist daher auch insbesondere davon abhängig, wie gut die zur Berechnung der Tragfähigkeit für die Beziehungen (4.11) bis (4.19) angenommenen Werte für die Einflußgrößen die in den unterschiedlichen Versuchsserien tatsächlich vorliegenden Mittelwerte dieser Einflußgrößen widergegeben. Die Abschätzungen, die zur Berechnung der Tragfähigkeit für die Werte der Einflußgrößen getroffen wurden (vgl. Tabelle 5.1), werden nachfolgend erläutert:

- Für die Blechdicke t der Unterkonstruktion wurde der in der Spalte 10 der Tabelle A.1 angegebene Mittelwert der Blechdicke verwendet. Da dieser Wert dem Mittelwert der Kernblechdicken entspricht, die an den für die Auszugversuche verwendeten Probekörper gemessenen wurden, stellt er die bestmögliche Abschätzung dar, die für die Blechdicke $t$ getroffen werden kann. 
- Als Werkstoffkennwert der Unterkonstruktion muß zur Anwendung des mechanischen Modells die Scherfestigkeit $\tau_{\mathrm{m}, \mathrm{MG}}$ des Muttergewindes abgeschätzt werden. In [30] werden in der Tabelle A9 für Muttern aus unlegierten Baustählen sowie für Muttern aus Aluminiumlegierungen Scherfestigkeiten in Abhängigkeit von den technischen Zugfestigkeiten der Werkstoffe angegeben. Die auf die technischen Zugfestigkeiten bezogenen Scherfestigkeiten $\alpha_{s}=\tau_{m} / R_{m}$ liegen nach diesen Angaben für unlegierte Baustähle in den Festigkeitsklassen S235 bis S355 in einem Bereich zwischen $0,59 \leq \alpha_{s} \leq 0,60$ und für Aluminiumlegierungen mit Zugfestigkeiten zwischen $180 \mathrm{~N} / \mathrm{mm}^{2}$ bis $290 \mathrm{~N} / \mathrm{mm}^{2}$ in einem Bereich zwischen $0,58 \leq \alpha_{s} \leq 0,61$. Aufgrund dieser Angaben wird zur Ermittlung der Scherfestigkeiten für die im Rahmen dieser Arbeit untersuchten Werkstoffe für die Unterkonstruktion - Stahl der Festigkeitsklassen S235 bis S355 und Aluminiumlegierungen mit Mindestzugfestigkeiten in einem Bereich zwischen $195 \mathrm{~N} / \mathrm{mm}^{2}$ bis $250 \mathrm{~N} / \mathrm{mm}^{2}$ - eine auf die Zugfestigkeit bezogene Scherfestigkeit von $\alpha_{s}=0,60$ angenommen, und für die Zugfestigkeiten werden die in der Spalte 19 der Tabelle A.1 angegebenen Zugfestigkeiten herangezogen.

- Als geometrische Kennwerte der Schrauben müssen zur Anwendung des mechanischen Modells repräsentative Werte für den Gewindeaußendurchmesser $d_{g}$, die Gewindesteigung $P$, den Flankenwinkel $\alpha$, die Gewindeabflachung $c$ und den Lochdurchmesser $d_{L}$ in der Unterkonstruktion, der bei Bohrschrauben vom größeren der beiden Werte aus dem Kerndurchmesser des Schraubengewindes $d_{k}$ und dem Bohrspitzendurchmesser $d_{B s}$ und bei gewindefurchende Schrauben durch den Vorbohrdurchmesser $d_{B v}$ bestimmt wird, abgeschätzt werden. Da nicht für alle Versuchsserien repräsentative Meßwerte für diese geometrische Einflußgrößen vorliegen (für die Gewindeabflachung c, den Flankenwinkel a und den Lochdurchmesser $d_{L}$ liegen in keinem Fall Meßwerte vor), und da, sofern Meßwerte vorliegen, auch nicht bekannt ist, ob die Messungen an Schrauben durchgeführt wurden, die aus der gleichen Charge stammen, wie die in den Versuchen verwendeten Schrauben, wurden für die geometrischen Kennwerte $d_{g}, P, \alpha, d_{k}$ und $d_{B s}$ die Mittelwerte der in der Tabelle A.2 in den Spalten 3 bis 12 angegebenen Toleranzangaben verwendet. Für gewindefurchende Schrauben wurde für den Lochdurchmesser $d_{\llcorner}$der in der Tabelle A.1 in der Spalte 8 angegebene Nennwert des Vorbohrdurchmessers $d_{B v}$ verwendet. Für die Gewindeabflachung c wurde für alle Schrauben ein Wert von $c=0,15 \mathrm{~mm}$ verwendet, da die FE-Berechnungen für die simulierten Versuchsserien unter Zugrundelegung dieses Wertes eine gute Übereinstimmung zwischen den erreichten Maximallasten und den Versagenslasten der Versuche gezeigt haben und die Variation der Gewindeabflachung von $\mathrm{C}=0,15 \mathrm{~mm}$ auf einen Wert $\mathrm{c}=0,10 \mathrm{~mm}$ einen maximalen Einfluß von $6 \%$ auf die in den FE-Berechnungen der simulierten Versuchsserien erreichten Traglasten hatte. 
- Als Werkstoffkennwert muß für die Schrauben die Scherfestigkeit $\tau_{\mathrm{m}, \mathrm{SG}}$ in den Gewindegängen des Schraubengewindes abgeschätzt werden. In [30] werden in der Tabelle 5.5/2 für Schrauben aus niedriglegierten Kohlenstoffstählen sowie für Schrauben aus nichtrostenden Stählen Scherfestigkeiten in Abhängigkeit von den technischen Zugfestigkeiten der Werkstoffe angegeben. Nach diesen Angaben beträgt die auf die technische Zugfestigkeit bezogene Scherfestigkeit $\alpha_{s}=\tau_{m} / R_{m}$ für Schrauben aus Kohlenstoffstählen der Festigkeitsklasse 10.9 nach DIN EN ISO 898-1 (Mindestzugfestigkeit $R_{m, \min }=1000 \mathrm{~N} / \mathrm{mm}^{2}$ ) $\alpha_{s}=0,62$ und für Schrauben aus Kohlenstoffstählen der Festigkeitsklasse 12.9 nach DIN EN ISO 898-1 (Mindestzugfestigkeit $R_{m, \min }=1200 \mathrm{~N} / \mathrm{mm}^{2}$ ) beträgt das Scherfestigkeitsverhältnis $\alpha_{s}=0,60$. Da die im Rahmen dieser Arbeit untersuchten Schrauben aus niedriglegierten Kohlenstoffstählen bezüglich ihrer Festigkeitseigenschaften in die Festigkeitsklassen 10.9 bis 12.9 nach DIN EN ISO 898-1 einzuordnen sind - die Zugfestigkeiten der Schrauben liegen gemäß den Abschätzungen im Kapitel 3, Tabelle 3.1 zwischen $919 \mathrm{~N} / \mathrm{mm}^{2}$ und $1476 \mathrm{~N} / \mathrm{mm}^{2}$ - wurde zur Ermittlung der Scherfestigkeiten für die Schrauben aus niedriglegierten Kohlenstoffstählen ein gemäß den Angaben in [30] mittleres Scherfestigkeitsverhältnis von $\alpha_{s}=0,61$ angenommen. Gemäß [30], Tabelle 5.5/2 beträgt des Scherfestigkeitsverhältnis für Schrauben aus nichtrostendem Stahl der Festigkeitsklasse 70 nach DIN EN ISO 3506-1 (Mindestzugfestigkeit $R_{m, m i n}=700 \mathrm{~N} / \mathrm{mm}^{2}$ ) $\alpha_{s}=0,72$ und für Schrauben aus nichtrostendem Stahl der Festigkeitsklasse 80 nach DIN EN ISO 3506-1 (Mindestzugfestigkeit $R_{m, m i n}=800 N / \mathrm{mm}^{2}$ ) beträgt das Scherfestigkeitsverhältnis $\alpha_{s}=0,68$. Da die im Rahmen dieser Arbeit untersuchten Schrauben aus nichtrostenden Stählen bezüglich ihrer Festigkeitseigenschaften in die Festigkeitsklassen 70 bis 80 nach DIN EN ISO 3506-1 einzuordnen sind - die Zugfestigkeiten der Schrauben liegen gemäß den Abschätzungen im Kapitel 3, Tabelle 3.1 zwischen $730 \mathrm{~N} / \mathrm{mm}^{2}$ und $1299 \mathrm{~N} / \mathrm{mm}^{2}$ - wurde zur Ermittlung der Scherfestigkeiten für die Schrauben aus nichtrostenden Stählen ein gemäß den Angaben in [30] mittleres Scherfestigkeitsverhältnis von $\alpha_{s}=0,70$ angenommen. Für die Zugfestigkeiten der Schrauben werden die in der Spalte 26 der Tabelle A.2 abgeschätzten Zugfestigkeiten herangezogen. Für diejenigen Schrauben, für die in der Spalte 26 der Tabelle A.2 aufgrund des Fehlens von Zugbruchversuchen keine Zugfestigkeiten aufgeführt sind, wurden die in der Tabelle 3.1 des Kapitels 3 angegebenen Mittelwerte der für den jeweiligen Schraubenwerkstoff abgeschätzten Zugfestigkeiten herangezogen $\left(R_{m, S G, M i t t e}=1190 N / \mathrm{mm}^{2}\right.$ für Schrauben aus einem niedriglegierten Kohlenstoffstahl und $R_{m, S G, M i t t e l}=930 N / m^{2}$ für Schrauben aus nichtrostendem Stahl). 
Tabelle 5.1: Festlegung von repräsentativen Werten für die Einflußgrößen

\begin{tabular}{|c|c|c|c|}
\hline \multicolumn{3}{|c|}{ Einflußgrößen } & Festlegungen \\
\hline \multirow{2}{*}{$\begin{array}{l}\text { Unter- } \\
\text { kon- } \\
\text { struk- } \\
\text { tion }\end{array}$} & & $\mathrm{t}$ & $t=t_{K}$ mit $t_{K}$ nach Tabelle A.1, Spalte 10 \\
\hline & \multicolumn{2}{|c|}{$\tau_{\mathrm{m}, \mathrm{MG}}=\alpha_{\mathrm{s}, \mathrm{MG}} \cdot \mathrm{R}_{\mathrm{m}, \mathrm{MG}}$} & $\alpha_{s, M G}=0,60$ und $R_{m, M G}$ nach Tabelle A.1, Spalte 19 \\
\hline \multirow{4}{*}{$\begin{array}{l}\text { Schrau- } \\
\text { be }\end{array}$} & \multirow{3}{*}{$\begin{array}{l}\text { Geo- } \\
\text { metrie }\end{array}$} & 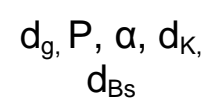 & $\begin{array}{c}\text { Mittelwert der Toleranzangaben } \\
\text { nach Tabelle A.2, Spalten } 3 \text { bis } 12\end{array}$ \\
\hline & & c & $\mathrm{c}=0,15 \mathrm{~mm}$ für alle Schrauben \\
\hline & & $d_{L}$ & $\begin{array}{c}\frac{\text { Bohrschrauben: }}{d_{L}=\text { Maximum }\left(d_{B s} ; d_{K}\right)} \\
\frac{\text { gewindefurchende Schrauben: }}{d_{L}=d_{B v} \text { mit } d_{B v} \text { nach Tabelle A.1, Spalte } 8}\end{array}$ \\
\hline & \multicolumn{2}{|c|}{$\tau_{\mathrm{m}, \mathrm{SG}}=\alpha_{\mathrm{s}, \mathrm{SG}} \cdot \mathrm{R}_{\mathrm{m}, \mathrm{SG}}$} & $\begin{array}{c}\text { Schrauben aus niedriglegierten Kohlenstoffstählen: } \\
\alpha_{\mathrm{s}, \mathrm{SG}}=0,61 \text { und } \mathrm{R}_{\mathrm{m}, \mathrm{SG}} \text { nach Tabelle A.2, Spalte } 26, \\
\text { sofern keine Angaben in Tabelle A.2: } R_{\mathrm{m}, \mathrm{SG}}=1190 \mathrm{~N} / \mathrm{mm}^{2} \\
\text { nichtrostende Schrauben: } \\
\alpha_{\mathrm{s}, \mathrm{SG}}=0,70 \text { und } \mathrm{R}_{\mathrm{m}, \mathrm{SG}} \text { nach Tabelle A.2, Spalte } 26, \\
\text { sofern keine Angaben in Tabelle A.2: } R_{\mathrm{m}, \mathrm{SG}}=930 \mathrm{~N} / \mathrm{mm}^{2}\end{array}$ \\
\hline
\end{tabular}

Zur Abschätzung der Versagenslasten für die einzelnen Versuchsserien wurde das im abschnitt 4.9.2 beschriebene mechanische Modell noch durch die nachfolgend erläuterten Erweiterungen ergänzt:

- Das Versagen $F_{A s, M G}$, das ausschließlich durch die Form und die Werkstoffestigkeit des Muttergewindes bestimmt ist, wurde mit Hilfe der Parameterstudien durch die Beziehungen (4.12) bis (4.17) beschrieben, über die auch der tragfähigkeitsmindernde Einfluß einer radialen Aufweitung des Muttergewindes erfaßt wird. Die radiale Aufweitung führt dabei für den Bereich $t_{1}=0,6 m m \leq t \leq t_{2}$ ( $t_{2}$ nach (4.14)) zu einer Abminderung der im Abschnitt 4.9.2.1 hergeleiteten Abschertragfähigkeit $\mathrm{F}_{\mathrm{As}, \mathrm{MG}}^{*}$ (Beziehungen (4.7) bis (4.10)) des Muttergewindes im Schnitt $r=d_{g} / 2$. Für Schrauben, für die aufgrund eines kleinen Lochdurchmessers $d_{L}$ in der Unterkonstruktion der Fall $t_{2}<4,90 \mathrm{~mm}$ vorliegt, ergaben sich für die größte Blechdicke $t=4,9 \mathrm{~mm}$, die in den Parameterstudien untersucht wurde, in den FE-Berechnungen mit der Werkstoffpaarung „CH_Al“ Tragfähigkeiten, die über $\mathrm{F}_{\text {As,MG }}^{*}$ liegen (die Maximallasten der FE-Berechnung liegen für diese Fälle maximal 14\% über $F^{*}{ }_{A s, M G}$, vgl. Diagramm D.26 im Anhang D). Für Blechdicken $t<t_{1}=0,60 \mathrm{~mm}$, die unter der kleinsten in den Parameterstudien untersuchten Blechdicke liegen, werden sich aufgrund der Tatsache, daß in diesem Blechdickenbereich der tragfähigkeitsmindernde Einfluß einer radialen Aufweitung des Muttergewindes keine Rolle mehr spielt, Tragfähigkeitswerte 
für das Versagen des Muttergewindes ergeben, die ebenfalls über $\mathrm{F}_{\mathrm{As}, \mathrm{MG}}^{*}$ liegen. Durch die Zugrundelegung der Beziehung (4.12) bis (4.17) für das Versagen, das ausschließlich durch das Muttergewinde bestimmt wird, ergeben sich für den Blechdickenbereich $\mathrm{t}_{1}=0,6 \mathrm{~mm} \leq \mathrm{t} \leq$ Maximum( $\left.\mathrm{t}_{2}, 4,90 \mathrm{~mm}\right)$ Tragfähigkeitswerte, die durch die Ergebnisse der Parameterstudien abgedeckt sind. Für Blechdicken $t>$ Maximum $\left(t_{2}, 4,90 \mathrm{~mm}\right)$ führt die Anwendung dieser Beziehungen jedoch zu Tragfähigkeitswerten, die bezogen auf den Wert $\mathrm{F}_{\mathrm{As}, \mathrm{MG}}^{\star}$ mit größer werdenden Blechdicke kontinuierlich ansteigen. Deshalb muß für Blechdicken $\mathrm{t}>$ Maximum $\left(\mathrm{t}_{2}, 4,90 \mathrm{~mm}\right)$ die Tragfähigkeit des Muttergewindes begrenzt werden. Dies wurde durch die nachfolgend angegebene Beziehung (5.1) erreicht, durch welche die Tragfähigkeit des Muttergewindes für Blechdicken $t>\operatorname{Maximum}\left(\mathrm{t}_{2}, 4,90 \mathrm{~mm}\right)$ auf den Wert $1,1^{*} \mathrm{~F}^{*}{ }_{\mathrm{As}, \mathrm{MG}}$ begrenzt wird. Für Blechdicken $\mathrm{t}<\mathrm{t}_{1}=0,6 \mathrm{~mm}$ führt die Anwendung der Beziehungen (4.12) bis (4.17) zu Tragfähigkeitswerten, die stets unter dem Wert $\mathrm{F}^{\star}{ }_{\mathrm{As}, \mathrm{MG}}$ liegen. Da der tragfähigkeitsmindernde Einfluß einer radialen Aufweitung des Muttergewindes für Blechdicken $\mathrm{t}<\mathrm{t}_{1}=0,6 \mathrm{~mm}$ keine Rolle mehr spielt, wird für Blechdicken $\mathrm{t}<\mathrm{t}_{1}=0,6 \mathrm{~mm}$ durch die Beziehungen (4.12) bis (4.17) die tatsächliche Tragfähigkeit des Muttergewindes unterschätzt. Diesem Sachverhalt wurde ebenfalls durch die Beziehung (5.1) Rechnung getragen, indem für Blechdicken $t<t_{1}=0,6 \mathrm{~mm}$ die Tragfähigkeit des Muttergewindes auf den Wert $1,1^{\star} \mathrm{F}_{\mathrm{As}, \mathrm{MG}}^{*}$ angehoben wurde. Da für alle untersuchten Schrauben für Blechdicken $t<t_{1}=0,60 \mathrm{~mm}$ die Bedingung $\varphi_{\mathrm{t}} \leq 2 \pi-\varphi_{\mathrm{C}}-\varphi_{\mathrm{EA}}$ erfüllt ist und für Blechdicken $t>$ Maximum( $\left.t_{2}, 4,90 \mathrm{~mm}\right)$ die Bedingung $\varphi_{t} \geq 2 \pi-\varphi_{c}$ erfüllt ist, ergeben sich die zur Berechnung von $\mathrm{F}_{\mathrm{As}, \mathrm{MG}}^{*}$ in der Beziehung (5.1) benötigten Abscherflächen aus den Beziehungen (4.9) und (4.7).

$\mathrm{F}_{\mathrm{As}, \mathrm{MG}}= \begin{cases}\text { für } \mathrm{t}<\mathrm{t}_{1}=0,60 \mathrm{~mm} & : 1,1 \cdot \mathrm{F}^{*}{ }_{\mathrm{As}, \mathrm{MG}} \text { mit } \mathrm{F}_{\mathrm{As}, \mathrm{MG}}^{*} \text { nach (4.10) und (4.9) } \\ \text { für } \mathrm{t}_{1} \leq \mathrm{t} \leq \operatorname{Max} .\left(\mathrm{t}_{2}, 4,90 \mathrm{~mm}\right): \mathrm{F}_{\mathrm{As}, \mathrm{MG}} \text { nach (4.12) bis (4.17) } \\ \text { für } \mathrm{t}>\operatorname{Max} .\left(\mathrm{t}_{2}, 4,90 \mathrm{~mm}\right) & : 1,1 \cdot \mathrm{F}^{*}{ }_{\mathrm{As}, \mathrm{MG}} \operatorname{mit}^{*}{ }_{\mathrm{As}, \mathrm{MG}} \text { nach (4.10) und (4.7) }\end{cases}$

Da in der Versuchsdatenbank auch Versuchsserien aufgeführt sind, für die das Versagen durch einen Zugbruch der Schrauben stattgefunden hat, muß das im Abschnitt 4.9.2 entwickelte mechanische Modell um diesen Versagensfall ergänzt werden. Hierfür wurde die Zugbruchlast der Schrauben $F_{Z B}$ durch die Multiplikation des Schraubenkernquerschnittes im Gewindebereich mit der in der Tabelle A.2 in der Spalte 26 angegebenen Zugfestigkeit $R_{m, S G}$ abgeschätzt (vgl. Beziehung (5.2)). Für diejenigen Schrauben, für die in der Spalte 26 der Tabelle A.2 keine Zugfestigkeiten angegeben sind, wurden die in der Tabelle 3.1 des Kapitels 3 angegebenen Mittelwerte der für den jeweiligen Schraubenwerkstoff abgeschätzten Zugfestigkeiten herangezogen $\left(R_{m, S G, M i t t l}=1190 N / m^{2}\right.$ für Schrauben aus einem niedriglegierten Kohlenstoffstahl und $R_{m, S G, \text { Mittel }}=930 \mathrm{~N} / \mathrm{mm}^{2}$ für Schrauben aus nichtrostendem Stahl). 
$\mathrm{F}_{\mathrm{ZB}}=\pi \cdot \frac{\mathrm{d}_{\mathrm{K}}^{2}}{4} \cdot \mathrm{R}_{\mathrm{m}, \mathrm{SG}}$

- Mit Hilfe des durch die Beziehungen (5.1) und (5.2) erweiterten mechanischen Modells kann über die Beziehung (5.3) für jede der in der Versuchsdatenbank aufgeführten Versuchsserien ein mittlere Versagenslast $r_{t \text {,mittel }}$ berechnet werden.

$\mathrm{r}_{\mathrm{t} \text {,mittel }}=\operatorname{Minimum}\left(\mathrm{F}_{\mathrm{AZV}}, \mathrm{F}_{\mathrm{ZB}}\right) \quad$ mit : $\left\{\begin{array}{l}\mathrm{F}_{\mathrm{AZV}} \operatorname{nach}(4.11) \text { bis (4.19) und (5.1) } \\ \mathrm{F}_{\mathrm{ZB}} \operatorname{nach}(5.2)\end{array}\right.$

Die berechneten Werte $r_{t \text {,mittel }}$ sind im Diagramm 5.1 über den in den Versuchsserien erreichten mittleren Versagenslasten $r_{v \text {,mittel }}$ aufgetragen. Die berechneten Werte $r_{t, \text { mittel }}$ liegen im Mittel 2\% unter und maximal 30\% über und 33\% unter den in den Versuchsserien erreichten mittleren Versagenslasten $r_{v \text {,mittel. }}$

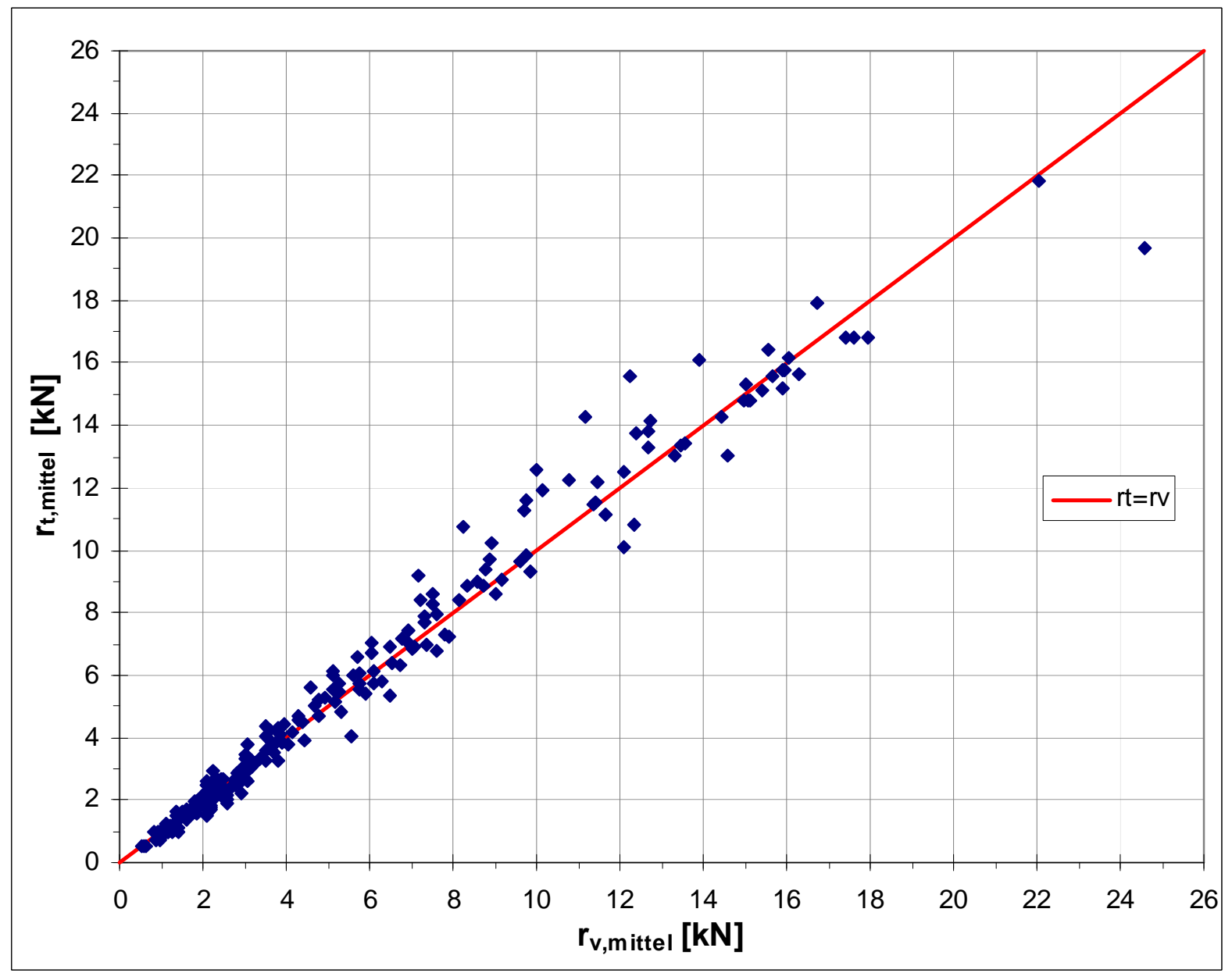

Diagramm 5.1: Vergleich der berechneten Tragfähigkeiten $r_{t, \text { mittel }}$ mit den in den Versuchsserien erreichten mittleren Versagenslasten $r_{v, \text { mittel }}$ 


\subsection{Charakteristischer Wert der Beanspruchbarkeit}

Die festgestellten Abweichungen zwischen den in den Versuchen erreichten mittleren Versagenslasten $r_{v \text {,mittel }}$ und den berechneten Tragfähigkeiten $r_{t, \text { mittel }}$ ist durch die Streuungen der in das Berechnungsmodell eingehenden Einflußgrößen zu erklären. Diese Streuungen machen sich insbesondere daher stark bemerkbar, weil die zur Berechnung der Tragfähigkeiten angenommenen Mittelwerte der Einflußgrößen, die durch den Werkstoff und die Geometrie der Schrauben bestimmt werden, größtenteils nicht durch Messungen bestätigt sind, die an den für die Auszugversuche verwendeten Proben oder zumindest an Proben durchgeführt wurden, die aus der gleichen Charge stammen, wie die in den Auszugversuchen verwendeten Proben. Zur Festlegung des charakteristischen Wertes der Beanspruchbarkeit wurden die Streuungen der Einflußgrößen sowie eventuell vorliegende Unsicherheiten im verwendeten mechanischen Modell durch die nachfolgend erläuterte Vorgehensweise berücksichtigt.

- Die Variable $\mathrm{X}=\mathrm{r}_{\mathrm{v}, \text { mittel }} / \mathrm{r}_{\mathrm{t} \text {,mittel, }}$, die sich aus dem Quotienten der mittleren Versagenslast einer Versuchsserie und der berechneten Tragfähigkeit für diese Versuchsserie ergibt, wird als Zufallsvariable angesehen, deren Verteilungsfunktion durch eine Normalverteilung angenähert wird.

- Zur Berücksichtigung des Sachverhaltes, daß die Variable x für eine Versuchsserie aus $\mathrm{n}_{\text {AZV }}$ (vgl. Versuchsdatenbank Tabelle A.1, Spalte 9) Einzelversuchen resultiert und daher Versuchsserien mit einer größeren Anzahl von Einzelversuchen eine größere statistische Zuverlässigkeit besitzen als Versuchsserien mit einer kleineren Anzahl von Einzelversuchen, wurde die Variable $\mathrm{x}$ mit der Wertigkeit $\mathrm{n}_{\mathrm{AzV}}$ belegt. Dies bedeutet für die Variable $\mathrm{x}$ steht in der Versuchsdatenbank ein Stichprobenumfang von insgesamt $n=\sum n_{A Z V}=2176$ Ergebnissen zur Verfügung wobei die Variable $x$ für eine Versuchsserie $n_{A z V}-M a l$ den gleichen Wert einnimmt.

- $\quad$ Mit Hilfe der Stichprobe wurde für die Variable $x$ der Farktilwert $x_{\text {Fraktil }}$ berechnet der lediglich in $\mathrm{p}=5 \%$ aller Fälle mit einer Aussagewahrscheinlichkeit von $\mathrm{pA}=75 \%$ unterschritten wird. Dieser Fraktilwert wird bei Versuchsauswertungen im Bereich des Bauwesens üblicherweise zur Bestimmung des charakteristischen Wertes einer Beanspruchbarkeit herangezogen (vgl. [45]). Der Fraktilwert $x_{\text {Fraktil }}$ ergibt sich mit dem Mittelwert $x_{m}$ und der Standardabweichung $s_{x}$ der Stichprobe sowie dem Fraktilfaktor $K_{s}(n, p=5 \%, p A=75 \%)$ aus der Beziehung (5.4). Zahlenwerte für den Fraktilfaktor $K_{S}$ für die 5\%-Fraktile und eine Aussagewahrscheinlichkeit von $75 \%$ sind in Abhängigkeit vom Stichprobenumfang $\mathrm{n}$ dem einschlägigen Schrifttum zu entnehmen (z.B. [45] und [46]).

$x_{\text {Fraktil }}=x_{m}-K_{S}(n, p=5 \%, p A=75 \%) \cdot S_{x}$ 
- Mit (5.4) ergibt sich der Fraktilwert $r_{t, \text { Fraktil }}$ der Beanspruchbarkeit, der lediglich in $5 \%$ aller Fälle mit einer Aussagewahrscheinlichkeit von 75\% unterschritten wird, aus der Beziehung (5.5).

$r_{t, \text { Fraktil }}=X_{\text {Fraktil }} \cdot r_{t, \text { mittel }}$

In der Tabelle 5.2 sind die Ergebnisse der oben beschriebenen Vorgehensweise aufgeführt. Die Auswertung nach dieser Vorgehensweise erfolgte

- einmal mit dem gesamten Stichprobenumfang, unabhängig davon, ob für die berechneten Tragfähigkeiten das Auszugversagen oder das Zugbruchversagen maßgebend wurde,

- einmal nur mit dem Stichprobenumfang, für den das Auszugversagen für die berechneten Tragfähigkeiten maßgebend wurde $\left(r_{t, \text { mittel }}=F_{A Z V}<F_{Z B}\right.$ in (5.3)) und

- einmal nur mit dem Stichprobenumfang, für den das Zugbruchversagen für die berechneten Tragfähigkeiten maßgebend wurde $\left(r_{t, \text { mittel }}=F_{Z B}<F_{A Z V}\right.$ in (5.3)).

Tabelle 5.2: Ergebnisse der Auswertungen zur Bestimmung des Fraktilwertes der Beanspruchbarkeit

\begin{tabular}{|c|c|c|c|c|c|c||}
\hline $\begin{array}{c}\text { ausgewertete } \\
\text { Versuche }\end{array}$ & $\mathrm{n}$ & $\mathrm{K}_{\mathrm{s}}$ & $\mathrm{x}_{\mathrm{m}}$ & $\mathrm{s}_{\mathrm{x}}$ & $\mathrm{x}_{\text {Fraktil }}$ & $\mathrm{r}_{\mathrm{t}, \text { Fraktil }}$ \\
\hline \hline alle & 2176 & 1,667 & 1,024 & 0,133 & 0,802 & $0,80 \cdot \mathrm{r}_{\mathrm{t}, \text { mittel }}$ \\
\hline $\mathrm{r}_{\mathrm{t}, \text { mittel }}=\mathrm{F}_{\mathrm{AZV}}<\mathrm{F}_{\mathrm{ZB}}$ & 1993 & 1,671 & 1,029 & 0,136 & 0,802 & $0,80 \cdot \mathrm{F}_{\mathrm{AZV}}$ \\
\hline $\mathrm{r}_{\mathrm{t}, \text { mittel }}=\mathrm{F}_{\mathrm{ZB}}<\mathrm{F}_{\mathrm{AZV}}$ & 183 & 1,730 & 0,968 & 0,073 & 0,841 & $0,84 \cdot \mathrm{F}_{\mathrm{ZB}}$ \\
\hline
\end{tabular}

Aus den Auswertungen wird deutlich, daß der Fraktilwert $x_{\text {Fraktil }}$ für das Zugbruchversagen ca. $5 \%$ über dem Fraktilwert für das Auszugversagen liegt. Dieses Ergebnis wird verständlich aus dem Sachverhalt, daß zur Beschreibung des Zugbruchversagens im Vergleich zu dem Auszugversagen weniger Einflußgrößen (nämlich lediglich der Kerndurchmesser $d_{k}$ der Schraube und die Zugfestigkeit $R_{m . s G}$ des Schraubengewinde) für das mechanische Modell benötigt werden und somit der Effekt der Streuungen der Einflußgrößen für das Zugbruchversagen im Vergleich zu dem Auszugversagen geringer ist. Mit Hilfe der Ergebnisse der Tabelle 5.2 und den Festlegungen der Tabelle 5.1 für die Werte der Einflußgrößen ergibt sich der Fraktilwert der Beanspruchbarkeit aus der Beziehung (5.6).

$\mathrm{r}_{\mathrm{t}, \text { Fraktil }}=\operatorname{Minimum}\left(0,80 \cdot \mathrm{F}_{\mathrm{AZV}}, 0,84 \cdot \mathrm{F}_{\mathrm{ZB}}\right) \quad \operatorname{mit}:\left\{\begin{array}{l}\mathrm{F}_{\mathrm{AZV}} \operatorname{nach}(4.11) \text { bis }(4.19) \text { und (5.1) } \\ \mathrm{F}_{\mathrm{ZB}} \operatorname{nach}(5.2)\end{array}\right.$ 
Im Diagramm 5.2 sind die berechneten Werte $r_{t, \text { Fraktil }}$ über die in den Versuchsserien erreichten mittleren Versagenslasten $r_{v}$,mittel aufgetragen. Die berechneten Werte $r_{t, F r a k t i l}$ liegen maximal nur $6 \%$ über den in den Versuchsserien erreichten mittleren Versagenslasten $r_{v, \text { mittel. }}$

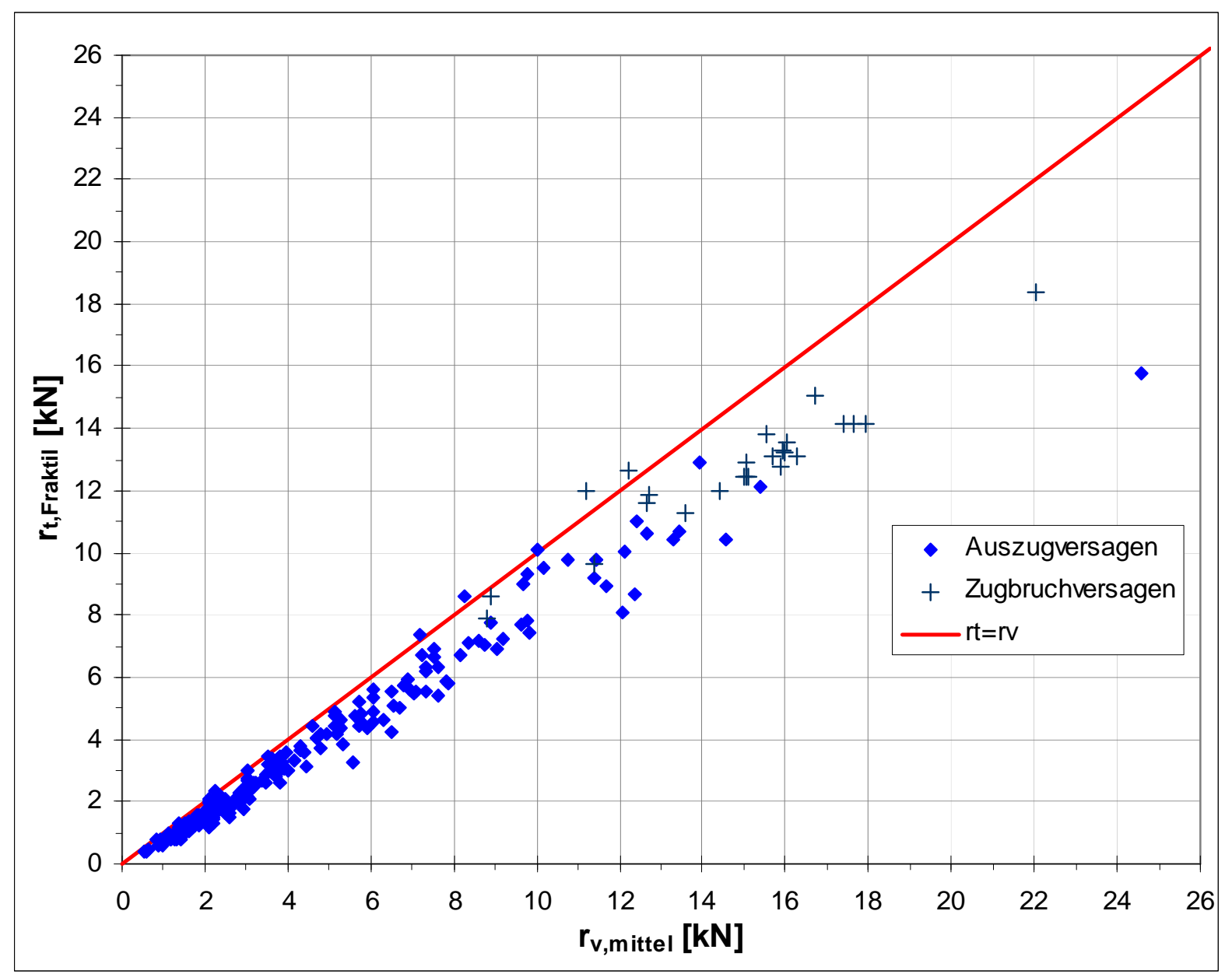

Diagramm 5.2: Vergleich der berechneten Fraktilwerte $r_{t, \text { Fraktil }}$ mit den in den Versuchsserien erreichten mittleren Versagenslasten $r_{v \text {,mittel }}$

Der Fraktilwert $r_{t, \text { Fraktil }}$ entspricht dem charakteristischen Wert der Beanspruchbarkeit $r_{t, k}$, sofern bei der Bemessung in der Berechnungsvorschrift (5.6) für die Einflußgrößen Werte verwendet werden, die den in der Tabelle 5.1 abgeschätzten Werten entsprechen oder zumindest eine auf der sicheren Seite liegende Abschätzung dieser Werte darstellen.

Aus dieser Überlegung heraus werden zur Ermittlung des charakteristischen Wertes der Beanspruchbarkeit für die in die Berechnungsvorschrift eingehenden Einflußgrößen folgende Festlegungen getroffen:

- Für die geometrischen Einflußgrößen der Schrauben $d_{g}, P, \alpha, d_{k}, d_{B s}$ werden gemäß den Angaben in der Tabelle 5.1 die Mittelwerte der Toleranzangaben und für die Gewindeabflachung $\mathrm{c}$ wird für alle Schrauben der Wert $\mathrm{c}=0,15 \mathrm{~mm}$ in die Berechnungsvorschrift eingesetzt. Darüber hinaus wird für gewindefurchende Schrauben für den Lochdurchmesser $d_{L}$ in der Unterkonstruktion der Nennwert des Vorbohrdurchmessers $d_{B V}$ verwendet. 
- Zur Ermittlung der Scherfestigkeiten des Muttergewindes $\tau_{\mathrm{m}, \mathrm{MG}}$ und des Schraubengewindes $\tau_{\mathrm{m}, \mathrm{SG}}$ werden die Scherfestigkeitsverhältnisse $\alpha_{\mathrm{s}, \mathrm{MG}}=0,6$ für Unterkonstruktionen aus Stahl und Aluminiumlegierungen und $\alpha_{s, S G}=0,61$ für Schrauben aus niedriglegierten Kohlenstoffstählen sowie $\alpha_{\mathrm{s}, \mathrm{SG}}=0,70$ für Schrauben aus nichtrostenden Stählen herangezogen.

- Zur Abschätzung der Zugfestigkeiten des Schraubengewindes $R_{m, s G}$ müssen für einen Schraubentyp Zugbruchversuche vorliegen, deren Versagen durch einen Zugbruch der Schrauben im Gewindebereich stattgefunden hat. Die in die Berechnungsvorschrift einzusetzende Zugfestigkeit der Schrauben ergibt sich dann aus dem Quotienten gebildet aus dem Mittelwert der Zugbruchtragfähigkeiten und dem Schraubenkernquerschnitt. Für den Kerndurchmesser des Schraubengewindes ist der Mittelwert der Toleranzangaben des Herstellers zu verwenden.

- Für die Blechdicke $t$ sowie für die Zugfestigkeit $R_{m}$ der Unterkonstruktion konnten zur Ermittlung des Fraktilwertes der Beanspruchbarkeit mit Hilfe der in der Versuchsdatenbank vorliegenden Meßwerte die im Mittel tatsächlich vorhandenen Werte dieser Einflußgrößen gut abgeschätzt werden. Für die Bemessung stehen dem Anwender diese Mittelwerte nicht zur Verfügung. Als eine konservative Abschätzung für die Blechdicke $t$ und für die Zugfestigkeit $R_{m}$ werden die in der jeweiligen Produktnorm für die Erzeugnisform und für den Werkstoff spezifizierten Mindestdicken $t_{\text {min }}$ und Mindestzugfestigkeiten $R_{m \text {,min }}$ in die Berechnungsvorschrift eingesetzt.

Der charakteristische Wert der Beanspruchbarkeit $r_{t, k}$ ergibt sich aus der Berechnungsvorschrift (5.6), in dem in (5.6) für die Werte der Einfußgrößen die oben beschriebenen Festlegungen berücksichtigt werden.

Der Berechnungsgang zur Ermittlung von $\mathrm{r}_{\mathrm{t}, \mathrm{k}}$ ist im Bild 5.1 zusammenfassend dargestellt. 


\section{Tragfähigkeit $\boldsymbol{F}_{A s, S G}$ des Schraubengewindes:}

$\mathrm{F}_{\mathrm{As}, S G}=1,2 \cdot \alpha_{s, S G} \cdot R_{m, S G}\left[\mathrm{c}+\left(\mathrm{d}_{\mathrm{g}}-\mathrm{d}_{\mathrm{L}}\right) \cdot \tan \left(\frac{\alpha}{2}\right)\right] \cdot \mathrm{d}_{\mathrm{L}} \cdot \frac{\mathrm{t} \cdot \pi}{\mathrm{P}}$

Tragfähigkeit $\boldsymbol{F}_{A s, M G}$ des Muttergewindes:

$\mathrm{F}_{\mathrm{As}, \mathrm{MG}}= \begin{cases}1,1 \cdot \mathrm{F}^{*} \text { As,MG } & \text { für } \mathrm{t}<\mathrm{t}_{1} \text { oder } \mathrm{t}>\text { Max. }\left(\mathrm{t}_{2}, 4,90 \mathrm{~mm}\right) \\ \mathrm{a} \cdot \mathrm{t}^{\mathrm{b}} & \text { für } \mathrm{t}_{1} \leq \mathrm{t} \leq \text { Max. }\left(\mathrm{t}_{2}, 4,90 \mathrm{~mm}\right)\end{cases}$

mit:

$\mathrm{t}_{1}=0,60 \mathrm{~mm} \quad(5.11) \quad ; \quad \mathrm{t}_{2}= \begin{cases}0,9 \cdot \mathrm{d}_{\mathrm{L}} & \text { für } \mathrm{d}_{\mathrm{L}} \leq 4 \mathrm{~mm} \\ \mathrm{~d}_{\mathrm{L}} & \text { für } 4 \mathrm{~mm}<\mathrm{d}_{\mathrm{L}} \leq 5 \mathrm{~mm} \\ 1,1 \cdot \mathrm{d}_{\mathrm{L}} & \text { für } \mathrm{d}_{\mathrm{L}}>5 \mathrm{~mm}\end{cases}$

$\mathrm{F}_{\mathrm{As}, M G}=\left\{\begin{array}{l}\alpha_{s, M G} \cdot \mathrm{R}_{\mathrm{m}, \text { min }} \cdot \frac{\mathrm{d}_{\mathrm{g}}}{2}\left[\frac{\pi}{\mathrm{P}} \cdot \mathrm{t}^{2}+\mathrm{t} \cdot \varphi_{\mathrm{EA}}\right] \text { für } \mathrm{t} \leq \mathrm{t}_{1} \\ \alpha_{s, M G} \cdot \mathrm{R}_{\mathrm{m}, \text { min }} \cdot \frac{\mathrm{d}_{\mathrm{g}}}{2}\left[\left(2 \pi-\varphi_{\mathrm{c}}\right)^{2} \frac{\mathrm{P}}{4 \pi}+(\mathrm{P}-\mathrm{c}) \cdot\left(\frac{2 \pi}{\mathrm{P}} \mathrm{t}-2 \pi+\varphi_{\mathrm{c}}\right)+\varphi_{\mathrm{EA}} \cdot\left(\mathrm{P}-\mathrm{c}-\frac{\mathrm{P}}{4 \pi} \varphi_{\mathrm{EA}}\right)\right] \text { für } \mathrm{t} \geq \mathrm{t}_{2}\end{array}\right.$

$\mathrm{a}=\frac{0,95 \cdot \mathrm{F}^{*} \mathrm{As}, \mathrm{MG}\left(\mathrm{t}=\mathrm{t}_{1}\right)}{\mathrm{t}_{1}{ }^{\mathrm{b}}}$

$$
\mathrm{b}=\frac{\ln \left(\frac{\mathrm{F}^{*} \mathrm{As,MG}\left(\mathrm{t}=\mathrm{t}_{2}\right)}{0,95 \cdot \mathrm{F}^{*} \mathrm{As}, \mathrm{MG}\left(\mathrm{t}=\mathrm{t}_{1}\right)}\right)}{\ln \left(\frac{\mathrm{t}_{2}}{\mathrm{t}_{1}}\right)}
$$

Abminderung zur Berücksichtigung des kombinierten Versagens:

$\mathrm{V}_{\mathrm{MG}, \mathrm{SG}}=\frac{\mathrm{F}_{\mathrm{As}, \mathrm{MG}}}{\mathrm{F}_{\mathrm{As}, \mathrm{SG}}}$

$\mathrm{R}\left(\mathrm{V}_{\mathrm{MG}, \mathrm{SG}}\right)= \begin{cases}1+0,30 \cdot \mathrm{e}^{\left(1,95 \cdot\left(1-\frac{1}{\mathrm{~V}_{\mathrm{MG}, S G}}\right)\right)} & \text { für } \mathrm{V}_{\mathrm{MG}, \mathrm{SG}} \leq 1 \\ 1+0,30 \cdot \mathrm{e}^{\left(2,5 \cdot\left(1-\mathrm{V}_{\mathrm{MG}, S G}\right)\right)} & \text { für } \mathrm{V}_{\mathrm{MG}, \mathrm{SG}} \geq 1\end{cases}$

Auszugstragfähigkeit:

$\mathrm{F}_{\mathrm{AZV}}=\frac{\operatorname{Minimum}\left(\mathrm{F}_{\mathrm{As}, \mathrm{SG}}, \mathrm{F}_{\mathrm{As}, \mathrm{MG}}\right)}{\mathrm{R}\left(\mathrm{V}_{\mathrm{MG}, \mathrm{SG}}\right)}$

\section{Zugbruchtragfähigkeit der Schraube:}

$\mathrm{F}_{\mathrm{ZB}}=\pi \cdot \frac{\mathrm{d}_{\mathrm{K}}^{2}}{4} \cdot \mathrm{R}_{\mathrm{m}, \mathrm{SG}}$

Charakteristischer Wert der Beanspruchbarkeit:

$\mathrm{r}_{\mathrm{t}, \mathrm{k}}=\operatorname{Minimum}\left(0,80 \cdot \mathrm{F}_{\mathrm{AZV}}, 0,84 \cdot \mathrm{F}_{\mathrm{ZB}}\right)$

\section{Parameter für die Schrauben:}

\section{Geometrie:}

- für $d_{g}, d_{K}, d_{B s}, P$ und $\alpha$ sind die Mittelwerte der Toleranzangaben zu verwenden

- $\mathrm{C}=0,15 \mathrm{~mm}$

- $\quad \mathrm{d}_{\mathrm{L}}=\left\{\begin{array}{l}\text { Bohrschrauben : Max. }\left(\mathrm{d}_{\mathrm{K}}, \mathrm{d}_{\mathrm{Bs}}\right) \\ \text { gewindefurchende Schrauben }\end{array}\right.$

(

- $\varphi_{\mathrm{EA}}=\left(\mathrm{d}_{\mathrm{g}}-\mathrm{d}_{\mathrm{L}}\right) \cdot \tan \left(\frac{\alpha}{2}\right) \cdot \frac{\pi}{\mathrm{P}} ; \varphi_{\mathrm{c}}=\mathrm{c} \cdot \frac{2 \pi}{\mathrm{P}}$

Werkstoff:

- $R_{m, S G}$ aus (5.19) und $F_{Z B}=$ Mittelwert der Versagenslasten aus Zugbruchversuchen

- $\alpha_{s, S G}=\frac{\tau_{\mathrm{m}, \mathrm{SG}}}{\mathrm{R}_{\mathrm{m}, \mathrm{SG}}}=\left\{\begin{array}{l}0,70 \text { für nichtrostende Schrauben } \\ 0,61 \text { für Schrauben aus Kohelstoffstahl }\end{array}\right.$
Parameter für die Unterkonstruktion:

\section{Blechdicke:}

- $\quad$ t..................garantierte Mindestkernblechdicke

Werkstoff:

- $\mathrm{R}_{\mathrm{m}, \mathrm{min}} \ldots \ldots \ldots . .$. garantierte Mindestzugfestigkeit

$\alpha_{s, M G}=\frac{\tau_{\mathrm{m}, \mathrm{MG}}}{\mathrm{R}_{\mathrm{m}, \min }}=0,60$ 


\section{$6 \quad$ Zusammenfassung}

In dieser Arbeit wurde für mit Hilfe von gewindeformender Schrauben ausgeführter Verbindungen des Metalleichtbaus eine Berechnungsvorschrift zur Bestimmung des charakteristischen Wertes der Auszugsbeanspruchbarkeit der Schrauben aus der Unterkonstruktion entwickelt. Diese Berechnungsvorschrift ist anwendbar für

- Stahl- und Aluminiumunterkonstruktionen,

- Bohrschrauben mit breiter und reduzierter Bohrspitze,

- gewindefurchende Schrauben,

- Schrauben aus nichtrostendem Stahl und

- Schrauben aus niedriglegierten Kohlenstoffstählen.

Grundlage der Bemessungsvorschrift waren numerische Berechnungen mit einem in dieser Arbeit entwickelten FE-Modell, in dem die räumliche Verzahnung zwischen dem Schraubengewinde und dem durch die Schraube in der Unterkonstruktion geformten Muttergewinde über die Verwendung von Volumenelementen und flächigen Kontaktelementen wirklichkeitsnah abgebildet wurde. Zur Beschreibung des Werkstoffverhaltens der Unterkonstruktionen sowie der Gewindegänge der Schrauben wurden nichtlineare Werkstoffgesetze für das FEModell verwendet, mit deren Hilfe die Verformungsverhältnisse im Verzahnungsbereich zwischen dem Schraubengewinde und dem Muttergewinde ebenfalls wirklichkeitsnah abgebildet werden konnten. Die Kalibrierung und Verifizierung des FE-Modells erfolgte durch eine Simulation von Auszugversuchsserien, die aus einer im Rahmen dieser Arbeit erstellten Versuchsdatenbank, in der 230 Auszugversuchsserien mit insgesamt 2176 Einzelversuchen aufgeführt sind, so ausgewählt wurden, daß sowohl für die Schrauben als auch für die Unterkonstruktionen eine möglichst große Bandbreite an unterschiedlichen geometrischen und werkstofflichen Randbedingungen abgedeckt waren. Mit Hilfe von Parameterstudien, die mit dem kalibrierten FE-Modell durchgeführt wurden, konnten die folgenden für das Auszugversagen maßgebenden Versagensmechanismen erfaßt und formelmäßig beschrieben werden.

- Das Versagen, das ausschließlich durch die Gewindegeometrie und die Werkstoffestigkeit der Schraube bestimmt wird, ist dadurch gegeben, daß die Gewindegänge der Schraube in dem radialen Schnitt, der dem Lochdurchmesser $d_{L}$ in der Unterkonstruktion entspricht, durch das Muttergewinde abgeschert werden.

- Das Versagen, das ausschließlich durch die Form und die Werkstoffestigkeit des Muttergewindes bestimmt wird, ist dadurch gegeben, daß die Gewindegänge des Muttergewin- 
des in dem radialen Schnitt, der dem Gewindeaußendurchmesser $d_{g}$ der Schraube entspricht, durch das Schraubengewinde abgeschert werden. Die radiale Aufweitung des Mutterkörpers führt hierbei zu einer gegenüber dem radial nicht aufgeweiteten Mutterkörper reduzierten Abscherfläche des Muttergewindes im Schnitt $r=d_{g} / 2$ und somit auch zu einer gegenüber dem radial nicht aufgeweiteten Muttergewinde reduzierten Auszugstragfähigkeit. Der tragfähigkeitsmindernde Effekt der radialen Aufweitung des Mutterkörpers ist für Blechdicken im Bereich von $1 \mathrm{~mm}$ bis $2 \mathrm{~mm}$ sowie für einen großen Lochdurchmesser $d_{\llcorner}$in der Unterkonstruktion besonders stark ausgeprägt.

- Das kombinierte Versagen von Schrauben und Muttergewinde ist dadurch bestimmt, daß sich im Grenzzustand des Versagens sowohl in den Gewindegängen des Schraubengewindes als auch in den Gewindegängen des Muttergewindes plastische Verformungen einstellen, die dazu führen, daß die Gewindegänge des Schraubengewindes und/oder die Gewindegänge des Muttergewindes in einem radialen Schnitt abgeschert werden, der zwischen dem Durchmesser $d_{L}$ des Kernloches in der Unterkonstruktion und dem AuBendurchmesser $d_{g}$ der Schraube liegt.

Zur Anwendung des anhand der Parameterstudien kalibrierten mechanischen Modells auf die Ergebnisse der Auszugversuche der Versuchsdatenbank wurde das mechanische Modell noch auf Blechdicken erweitert, die nicht durch die Parameterstudien abgedeckt waren, und der für die Auszugversuche mögliche Versagensfall, der durch einen Zugbruch der Schrauben bestimmt ist, wurde ebenfalls in das mechanische Modell integriert. Über den Vergleich zwischen den mit dem erweiterten mechanischen Modell berechneten Tragfähigkeiten und den in den Auszugversuchen der Versuchsdatenbank erreichten Versagenslasten wurde durch eine statistische Auswertung eine Berechnungsvorschrift zur Ermittlung des charakteristischen Wertes der Auszugsbeanspruchbarkeit entwickelt. Der charakteristische Wert der Beanspruchbarkeit ist aufgrund der statistischen Auswertungen als der 5\%-Fraktilwert der Tragfähigkeiten für eine Aussagewahrscheinlichkeit von $75 \%$ zu interpretieren. 


\section{Schrifttum}

[1] Schwarze, K; Lohmann F. A.: Konstruktion und Bemessung von Dach- und Wandflächen aus Stahl, Stahlbau-Kalender 1999

[2] Industrieverband zur Förderung des Bauens mit Stahlblech e. V., IFBSINFO 8.01: Richtlinie für die Montage von Stahlprofiltafeln für Dach-, Wand- und Deckenkonstruktionen, April 2002

[3] Industrieverband zur Förderung des Bauens mit Stahlblech e. V., IFBSINFO 7.02: Zulassungsbescheid für Verbindungselemente zur Verwendung bei Konstruktionen mit Sandwichelementen, DIBt-Zulassungsnummer Z-14.4-407, Dezember 2001

[4] Deutsches Institut für Bautechnik, Allgemeine bauaufsichtliche Zulassung Z-14.1-4: Verbindungselemente zur Verbindung von Bauteilen im Metalleichtbau, September 2005

[5] Klee, S.; Seeger, T.: Vorschlag zur vereinfachten Ermittlung von zulässigen Kräften für Befestigungen von Stahltrapezblechen, Veröffentlichung des Instituts für Statik und Stahlbau der Technischen Hochschule Darmstadt, Heft 33, Darmstadt, 1979

[6] European recommendations for steel construction, Publication No. 21: The design and testing of connections in steel sheeting and sections, ECCS Committee TC7, Working Group TWG 7.2, May 1983

[7] Großberndt, H.; Kayser, K.: Blechschrauben Handbuch, Vulkan-Verlag Dr. W. Classen Nachf. GmbH \& Co. KG, Essen 1968

[8] Großberndt, H.; Kniese, A.: Untersuchung über Querkraft- und Zugkraftbeanspruchungen sowie Folgerungen über kombinierte Beanspruchungen von Schraubenverbindungen bei Stahlprofilblech-Konstruktionen, Stahlbau 10/1975 und 11/1975, Oktober/ November 1975

[9] Bryan, E. R.; Sedlacek, G.; Tomà, A. W.; Weynand, K.: Evaluation of test results on connections in thin walled sheetings and members in order to obtain strength functions and suitable model factors, Background Document A.01 to Annex A of Eurocode No. 3, Institute of Steel Construction, RWTH Aachen, Germany, August 1990

[10] Tomà, A.; Sedlacek, G.; Weynand, K.: Connections in Cold-Formed Steel, ThinWalled Structures 16 (1993) 219-237, 1993 
[11] Rogowski, M.: Rechnerische Ermittlung der Tragfähigkeiten von geschraubten Verbindungen von Stahltrapezprofilen und Bauteilen aus Stahl, Vertieferarbeit, Lehrstuhl für Stahl- und Leichtmetallbau, TH Karlsruhe, Mai 1999

[12] Jülich, S.: Vergleich der im EC 3, Teil 1.3 angegebenen Formeln zur Berechnung der Tragfähigkeit für Verbindungen von kaltgeformten, dünnwandigen Bauteilen aus Stahl mittels Blechschrauben mit den Ergebnissen von Zulassungsversuchen zur Z-14.1-4 für derartige Verbindungen, Diplomarbeit, Lehrstuhl für Stahl- und Leichtmetallbau, TH Karlsruhe, Januar 2003

[13] Saal, H.; Hettmann, R.: Untersuchungen zur Querkrafttragfähigkeit von geschraubten Verbindungen in Konstruktionen mit Stahltrapezprofilen zur Überprüfung der Anwendbarkeit der im EC 3, Teil 1.3 angegebenen Formeln auf derartige Verbindungen, Bericht Nr. 021507, Versuchsanstalt für Stahl, Holz und Steine, TH Karlsruhe, Fraunhofer IRB Verlag, 2004

[14] Tang, R.B.; Mahendran, M.: Pull-out Strength of Steel Roof and Wall Cladding Systems, Journal of Structural Engineering, ASCE, Vol. 124, No. 10, 1998

[15] Herm, C.: Untersuchungen zum Tragverhalten von geschraubten Verbindungen im Stahlleichtbau, Vertieferarbeit, Lehrstuhl für Stahl- und Leichtmetallbau, TH Karlsruhe, Dezember 2001

[16] Baehre, R.; Holz, R.: Zulässige Zugkräfte von Verbindungen in AluminiumKonstruktionen mit gewindeformenden Schrauben, unveröffentlicht, Gutachten Nr. 890722, Karlsruhe, Juli 1989,

[17] Schardt, R.; Strehl, C.: Theoretische Grundlagen für die Bestimmung der Schubfestigkeit von Trapezblechscheiben - Vergleich mit anderen Berechnungsansätzen und Versuchsergebnissen, Stahlbau 4/1976, April 1976

[18] Schardt, R.; Strehl, C.: Stand der Theorie zur Bemessung von Trapezblechscheiben, Stahlbau 11/1980, November 1980

[19] Baehre, R.; Wolfram, R.: Zur Schubfeldberechnung von Trapezprofilen, Stahlbau 6/1986, 1986

[20] Baehre, R.: Zur Schubfeldwirkung und -bemessung von Kassettenkonstruktionen, Stahlbau 7/1987, 1987

[21] Deutsches Institut für Bautechnik, Allgemeine bauaufsichtliche Zulassung Z-14.1-88: ASTRON-Dachsystem PR-Dach, Januar 2002

[22] Deutsches Institut für Bautechnik, Allgemeine bauaufsichtliche Zulassung Z-14.1190: ASTRON-Dachsystem ASTROTEC-Dach, Januar 2002 
[23] Deutsches Institut für Bautechnik, Allgemeine bauaufsichtliche Zulassung Z-14.1473: RIB-ROOF Speed 500 Klemm-Falz-Profildach aus Stahl, März 2005

[24] Deutsches Institut für Bautechnik, Allgemeine bauaufsichtliche Zulassung Z-14.1381: SR- und SRC-Stahlkassettenprofiltafel-Konstruktion, Dezember 1998

[25] Schukey, H.; Hellwig, M.: Verbindungselemente im industriellen Leichtbau, Industrieverband zur Förderung des Bauens mit Stahlblech e. V., IFBS aktuell 1/00, 2000

[26] Steinhilper, W.; Röper R.: Maschinen- und Konstruktionselemente, Band 2, Verbindungselemente, 3. Auflage, Springer-Verlag, 1993

[27] Deutsches Institut für Bautechnik: Grundsätze für den Übereinstimmungsnachweis für Verbindungselemente im Metalleichtbau, DIBt Mitteilungen 6/1999, August 1999

[28] Friederich, H.: Werkstofftechnische Anforderungen an gewindeformende Verbindungselemente, DVM-Bericht 671, DVM-Tag 2004, Schraubenverbindungen, Mai 2004

[29] Wiegand H.; Kloos, K.-H.; Thomala, W.: Schraubenverbindungen, Grundlagen, Berechnung, Eigenschaften, Handhabung, 4. Auflage, Springer-Verlag, 1988

[30] VDI-Richtlinie 2230, Systematische Berechnung hochbeanspruchter Schraubenverbindungen, Zylindrische Einschraubenverbindungen, Verein Deutscher Ingenieure, Oktober 2001

[31] Thomala, W.: Beitrag zur Berechnung der Haltbarkeit von Schraubenköpfen mit Kraft-Innenangriff, VDI-Z Bd. 126 (1984) Nr. 9, S. 315-321, Mai 1984

[32] Weynand, K.: Sicherheits- und Wirtschaftlichkeitsuntersuchungen zur Anwendung nachgiebiger Anschlüsse im Stahlbau, Schriftenreihe Stahlbau - RWTH Aachen, Heft 35, Shaker Verlag, Aachen 1997

[33] Steurer, A.: Das Tragverhalten und Rotationsvermögen geschraubter Stirnplattenverbindungen, Institut für Baustatik und Konstruktion, ETH Zürich, IBK Bericht Nr. 247, Birkhäuser Verlag Basel, Dezember 1999

[34] Lacher, G.; Hedenkamp, A.: Zeit- und Dauerfestigkeit von hochfesten vorgespannten Schrauben in Stirnplattenstößen, Teil I und Teil II, Stahlbau 8/1993 und 9/1993, August/ September 1993

[35] Saal, H.; Hettmann, R.: Durchknöpftragfähigkeit von geschraubten Verbindungen in Konstruktionen mit gelochten Stahlblechen unter wiederholter Beanspruchung, Versuchsanstalt für Stahl, Holz und Steine, TH Karlsruhe, Fraunhofer IRB Verlag, 2005

[36] ANSYS Release 7.1; Manuals; ANSYS, Inc.; CAD-FEM GmbH, 2002 
[37] Allgemeine bauaufsichtliche Zulassung Z-30.3-6: Erzeugnisse, Verbindungsmittel und Bauteile aus nichtrostenden Stählen, Informationsstelle Edelstahl Rostfrei, Sonderdruck 862, Dezember 2003

[38] Schumann, P.; Kleineidam, P.; Seidel, M.: Zur FE-Modellierung von zugbeanspruchten Schraubenverbindungen, Stahlbau 2/2001, Februar 2001

[39] Wiegand, H.; Illgner, K. H.: Haltbarkeit von Schraubenverbindungen mit ISOGewindeprofil, Konstruktion Bd. 19 (1967) Nr. 3, 1967

[40] Wiegand, H.; Illgner, K. H; Strigens, P.: Einfluß der Gewindesteigung auf die Haltbarkeit von Schraubenverbindungen bei zügiger Beanspruchung, Industrie-Anzeiger Bd. 91 (1969) Nr. 85, 1969

[41] Alexander, E. A.: Analysis and Design of Threaded Assemblies, Int. Automotive Engineering, Congress and Exposition Detroit (1977), Rep. Nr. 770420, 1977

[42] Schneider, W.: Berechnung der Tragfähigkeit ineinandergreifender Gewinde, VDIBerichte Nr. 478, S. 55-62, VDI-Verlag GmbH, Düsseldorf 1983

[43] Thomala, W.: Zur Berechnung der erforderlichen Mutterhöhe bei Schraubenverbindungen, Konstruktion Bd. 47 (1995), 1995

[44] Thomala, W.: Beitrag zur Dauerhaltbarkeit von Schraubenverbindungen, Dissertation im Fachbereich Maschinenbau an der Technischen Hochschule Darmstadt, Dezember 1978

[45] Deutsches Institut für Bautechnik, Grundlagen zur Beurteilung von Baustoffen, Bauteilen und Bauarten im Prüf- und Zulassungsverfahren, 1986

[46] Struck, W.: Ermittlung des Bauteilwiderstandes aus Versuchsergebnissen bei vereinbartem Sicherheitsniveau, Forschungsbericht 58, Bundesanstalt für Materialprüfung (BAM), März 1979

[47] Kreyszig, E.: Statistische Methoden und ihre Anwendung, 4. Nachdruck der 7. Auflage, Vandenhoeck \& Ruprecht in Göttingen, 1991

[48] Plate, E. J.: Statistik und angewandte Wahrscheinlichkeitslehre für Bauingenieure, Ernst und Sohn Verlag für Architektur und technische Wissenschaften, 1993

[49] Unveröffentlichte Berichte der Versuchsanstalt für Stahl, Holz und Steine, TH Karlsruhe:

[a] Bericht Nr. 870350, Aufnehmbare Kräfte von Verbindungen mit gewindeformenden Schrauben in Aluminium-Konstruktionen, Oktober 1988 
[b] Bericht Nr. 890224, Tragfähigkeit von Verbindungen mit Bohrschrauben in Stahlleichtbau-Konstruktionen, Mai 1990

[c] Bericht Nr. 910104/1, Tragfähigkeitsuntersuchungen an Verbindungen mit nichtrostenden Bohrschrauben SXC 5 zur Befestigung von Sandwichelementen an Stahlkonstruktionen, Mai 1991

[d] Bericht Nr. 920047, Tragfähigkeitsuntersuchungen an Verbindungen mit nichtrostenden Bohrschrauben SD12-H15 zur Befestigung von Stahlblechprofilen an Stahlkonstruktionen, 1992

[e] Bericht Nr.920047 E, Ergänzende Tragfähigkeitsuntersuchungen an Verbindungen mit nichtrostenden Bohrschrauben SD12-H15 zur Befestigung von Stahlblechprofilen an Stahlkonstruktionen, September 1992

[f] Bericht Nr. 920125, Tragfähigkeitsuntersuchungen an Verbindungen mit nichtrostenden Bohrschrauben SX6 zur Befestigung von hochfesten Stahlblechprofilen an Stahlkonstruktionen, Juni 1992

[g] Bericht Nr. 920244, Experimentelle Untersuchungen zur Ermittlung der aufnehmbaren Quer- und Längszugkräfte von Verbindungsmitteln zur Befestigung in Stahlleichtbau-Konstruktionen, August 1992

[h] Bericht Nr. 920453, Tragfähigkeitsuntersuchungen an Verbindungen mit Bohrschrauben WÜRTH Color Pias 6,3-S und -R zur Befestigung von Stahlblechprofilen an Stahlkonstruktionen, März 1993

[i] Bericht Nr. 920462, Tragfähigkeitsuntersuchungen an Verbindungen mit nichtrostenden Bohrschrauben SX12 zur Befestigung von Stahlblechprofilen an Stahlkonstruktionen, März 1993

[j] Bericht Nr. 923572, Experimentelle Untersuchungen zur Ermittlung der aufnehmbaren Quer- und Längszugkräfte von Verbindungselementen zur Befestigung von Sandwichelementen an Stahlkonstruktionen, September 1993

[k] Bericht Nr. 933006, Experimentelle Untersuchungen zur Ermittlung der aufnehmbaren Quer- und Längszugkräfte von Verbindungsmitteln zur Befestigung in Stahlleichtbau-Konstruktionen, Juni 1993

[l] Bericht Nr. 934068, Auslenkversuche an in Stahl oder Holz eingeschraubten Edelstahlschrauben

[m] Bericht Nr. 943009, Experimentelle Untersuchungen zur Ermittlung der aufnehmbaren Quer- und Längszugkräfte von Verbindungsmitteln zur Befestigung in Stahlleichtbau-Konstruktionen, April 1994

[n] Bericht Nr. 943031, Experimentelle Untersuchungen zur Ermittlung der aufnehmbaren Quer- und Längszugkräfte von Verbindungselementen zur Befestigung in Stahlleichtbau-Konstruktionen, Dezember 1994 
[o] Bericht Nr. 943032, Experimentelle Untersuchungen zur Ermittlung der aufnehmbaren Quer- und Längszugkräfte von Verbindungselementen zur Befestigung in Stahlleichtbau-Konstruktionen, März 1995

[p] Bericht Nr. 953001, Tests For the Determination of the Shear And Tensile Strength of Connecting Elements in Light Steel Constructions, August 1995

[q] Bericht Nr. 953008, Experimentelle Untersuchungen zur Ermittlung der aufnehmbaren Quer- und Längszugkräfte von Verbindungselementen zur Befestigung in Stahlleichtbau-Konstruktionen, August 1995

[r] Bericht Nr. 953031, Experimentelle Untersuchungen zur Ermittlung der aufnehmbaren Quer- und Längszugkräfte von Verbindungselementen zur Befestigung in Stahlleichtbau-Konstruktionen, Oktober 1995

[s] Bericht Nr. 953052, Experimentelle Untersuchungen zur Ermittlung von Querzug-, Durchknöpf- und Auszugskräften von Sandwichbefestigungen mit den Verbindungselementen MAGE 7570 5,5/6,3 x L, MAGE 7575 5,5/6,3 x L,Mage 7580 6,3/7,0 x L auf Stahl- und Holzunterkonstruktionen, August 1996

[t] Bericht Nr. 963006, Experimentelle Untersuchungen zur Ermittlung der zulässigen Auslenkung des Verbindungselementes SFS TDB-S-6,3 x L, September 1996

[u] Bericht Nr. 963010, Experimentelle Untersuchungen zur Ermittlung der aufnehmbaren Querzug-, Durchknöpf- und Auszugskräften von Verbindungselementen zur Befestigung in Stahlleichtbau-Konstruktionen, März 1996

[v] Bericht Nr. 973519, Experimentelle Untersuchungen zur Ermittlung der zulässigen Auslenkung des Verbindungselementes MIVRAG 6,3 x L-16 A2, Oktober 1997

[w] Bericht Nr. 983504-01, Experimentelle Untersuchungen zur Ermittlung der aufnehmbaren Quer- und Längszugkräfte von Verbindungen mit der Bohrschraube Goldovis VIS AP 6,3xL, September 1999

[x] Bericht Nr. 983504-02, Experimentelle Untersuchungen zur Ermittlung der aufnehmbaren Quer- und Längszugkräfte von Verbindungen mit der Schraube FASTO INOX Typ A 6,5 x L, Januar 2000

[y] Bericht Nr. 983504-03, Experimentelle Untersuchungen zur Ermittlung der aufnehmbaren Quer- und Längszugkräfte von Verbindungen mit der Schraube FASTO INOX Typ B 6,3 x L, Januar 2000

[z] Bericht Nr. 983535, Experimentelle Untersuchungen zur Ermittlung der aufnehmbaren Quer- und Längszugkräfte von geschraubten Verbindungen in StahlleichtbauKonstruktionen, Oktober 1999

[aa] Bericht Nr. 993604, Experimentelle Untersuchungen zur Ermittlung der aufnehmbaren Querzug-, Auszug- und Durchknöpfkräften von geschraubten Verbindungen mit den Schrauben MAGE 7510 und MAGE 7325, Juli 1999 
[bb] Bericht Nr. 993609, Experimentelle Untersuchungen zur Ermittlung der aufnehmbaren Quer- und Längszugkräfte sowie der zulässigen Kopfauslenkungen von geschraubten Verbindungen mit dem Verbindungselement MAGE 7571, März 2000

[cc] Bericht Nr. 993626, Experimentelle Untersuchungen zur Ermittlung der aufnehmbaren Quer- und Längszugkräften sowie der zulässigen Kopfauslenkungen von geschraubten Verbindungen in Stahlleichtbau-Konstruktionen, August 2000

[dd] Bericht Nr. 003602, Experimentelle Untersuchungen zur Ermittlung der aufnehmbaren Quer- und Längszugkräfte von geschraubten Verbindungen mit den Bohrschrauben Zebra-Piasta 4,8 und Zebra-Piasta 6,3, April 2000

[ee] Bericht Nr. 003603, Experimentelle Untersuchungen zur Ermittlung der aufnehmbaren Quer- und Längszugkräfte von geschraubten Verbindungen mit den Schrauben JT3-6-6,3xL und JT3-3H-4,8xL, Dezember 2000

[ff] Bericht Nr. 003612, Experimentelle Untersuchungen zur Ermittlung der aufnehmbaren Quer- und Längszugkräften von geschraubten Verbindungen in StahlleichtbauKonstruktionen, Dezember 2000

[gg] Bericht Nr. 013603, Experimentelle Untersuchungen zur Ermittlung der aufnehmbaren Quer- und Längszugkräfte sowie der zulässigen Kopfauslenkungen von geschraubten Verbindungen in Stahlleichtbau-Konstruktionen, November 2001

[hh] Bericht Nr. 023606, Experimentelle Untersuchungen zur Ermittlung der aufnehmbaren Quer- und Längszugkräfte von geschraubten Verbindungen in StahlleichtbauKonstruktionen, Juli 2002

[ii] Bericht Nr. 023608, Experimentelle Untersuchungen zur Ermittlung der aufnehmbaren Quer- und Längszugkräfte von geschraubten Verbindungen in StahlleichtbauKonstruktionen, November 2002

[j] Bericht Nr. 023609, Experimentelle Untersuchungen zur Ermittlung der aufnehmbaren Auszugkräfte sowie der zulässigen Kopfauslenkungen von geschraubten Verbindungen mit der Schraube JT2-12-6,3×185, August 2002

[kk] Bericht Nr. 023613, Auszug-, Auslenk- und Qualitätssicherungsversuche mit Sandwichelementbefestiger, November 2002

[II] Bericht Nr. 033606, Experimentelle Untersuchungen zur Ermittlung der aufnehmbaren Quer- und Längszugkräfte sowie der zulässigen Kopfauslenkungen von geschraubten Verbindungen in Stahlleichtbau-Konstruktionen, September 2003

[mm] Bericht Nr. 033613, Experimentelle Untersuchungen zur Ermittlung der aufnehmbaren Quer- und Längszugkräfte von geschraubten Verbindungen in StahlleichtbauKonstruktionen, Februar 2004 
[nn] Bericht Nr. 033618, Experimentelle Untersuchungen zur Ermittlung der aufnehmbaren Quer- und Längszugkräfte von geschraubten Verbindungen in StahlleichtbauKonstruktionen, März 2004

[oo] Bericht Nr. 043609, Experimentelle Untersuchungen zur Ermittlung der aufnehmbaren Quer- und Auszugkräfte von Bohrschrauben zur Verwendung in Aluminium Konstruktionen, Januar 2005 


\section{$8 \quad$ Zitierte Normen}

DIN 7975: Blechschraubenverbindungen; Anwendung; Richtwerte für Kernlochdurchmesser, August 1989

DIN 7998: Gewinde und Schraubenenden für Holzschrauben, Februar 1975

DIN 17440: Nichtrostende Stähle; Technische Lieferbedingungen für gezogenen Draht, März 2001

DIN 18807, Teil 1: Trapezprofile im Hochbau; Stahltrapezprofile; Allgemeine Anforderungen, Ermittlung der Tragfähigkeitswerte durch Berechnung, Juni 1987

DIN 18807, Teil 3: Trapezprofile im Hochbau; Stahltrapezprofile; Festigkeitsnachweis und konstruktive Ausbildung, Juni 1987

DIN 18807, Teil 6: Trapezprofile im Hochbau; Aluminium-Trapezprofile und ihre Verbindungen; Ermittlung der Tragfähigkeitswerte durch Berechnung, September 1995

DIN 18807, Teil 7: Trapezprofile im Hochbau; Aluminium-Trapezprofile und ihre Verbindungen; Ermittlung der Tragfähigkeitswerte durch Versuche, September 1995

DIN 18807, Teil 8: Trapezprofile im Hochbau; Aluminium-Trapezprofile und ihre Verbindungen; Nachweise der Tragsicherheit und Gebrauchstauglichkeit, September 1995

DIN 18807, Teil 9: Trapezprofile im Hochbau; Aluminium-Trapezprofile und ihre Verbindungen; Anwendung und Konstruktion, Juni 1998

DIN EN 485, Teil 2: Aluminium und Aluminiumlegierungen; Bänder, Bleche und Platten; Mechanische Eigenschaften, September 2004

DIN EN 755, Teil 2: Aluminium und Aluminiumlegierungen; Stranggepreßte Stangen, Rohre und Profile; Mechanische Eigenschaften, August 1997

DIN EN 10025, Teil 2: Warmgewalzte Erzeugnisse aus Baustählen; Technische Lieferbedingungen für unlegierte Baustähle, April 2005

DIN EN 10263-4: Walzdraht, Stäbe und Draht aus Kaltstauch- und Kaltfließpressstählen; Technische Lieferbedingungen für Vergütungsstähle, Februar 2002

DIN EN 10263-5: Walzdraht, Stäbe und Draht aus Kaltstauch- und Kaltfließpressstählen; Technische Lieferbedingungen für nichtrostende Stähle, Februar 2002

DIN EN 10326: Kontinuierlich schmelztauchveredeltes Band und Blech aus Baustählen; Technische Lieferbedingungen, September 2004 
DIN EN ISO 898-1: Mechanische Eigenschaften von Verbindungselementen aus Kohlenstoffstahl und legiertem Stahl; Schrauben, November 1999

DIN EN ISO 1478: Blechschraubengewinde, Dezember 1999

DIN EN ISO 3506-1: Mechanische Eigenschaften von Verbindungselementen aus nichtrostenden Stählen; Schrauben, März 1998

DIN EN ISO 3506-4: Mechanische Eigenschaften von Verbindungselementen aus nichtrostenden Stählen; Blechschrauben, Oktober 2003

DIN EN ISO 7085: Mechanische und funktionelle Eigenschaften von einsatzgehärteten und angelassenen metrischen gewindefurchenden Schrauben, Januar 2000

DIN EN ISO 18265: Metallische Werkstoffe - Umwertung von Härtewerten, Februar 2004

Entwurf DIN 18807, Teil 4: Trapezprofile im Hochbau; Verbindungen zwischen Stahltrapezprofilen und Bauteilen aus Stahl; Ermittlung der Tragfähigkeit durch Versuche, 1986

Eurocode 3, Part 1.3: Design of steel structures; General rules; Supplementary rules for coldformed thin gauge members and sheeting, April 1996

prEN 1993-1-3: Design of steel structures; General rules; Supplementary rules for coldformed members and sheeting, September 2005

prEN 1999-1-4: Design of aluminium structures; Supplementary rules for cold-formed sheeting, May 2004

StBK-N5: Swedish Code for Light-Gauge Metal Structures, National Swedish Committee on Regulations for Steel Structures, Swedish Institute of Steel Construction, Publication 76, March 1988 


\section{Anhang A}

Versuchsdatenbank 


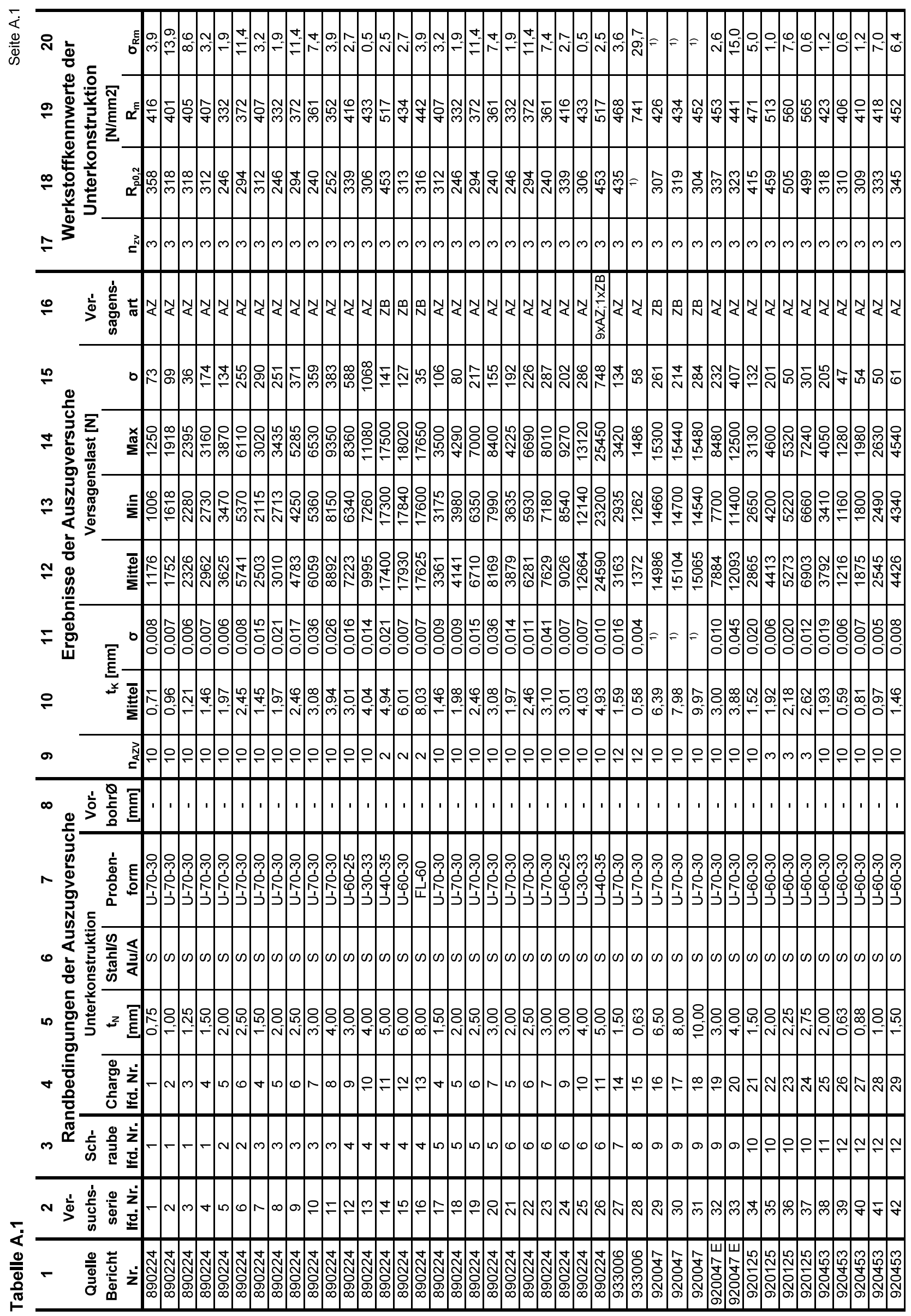




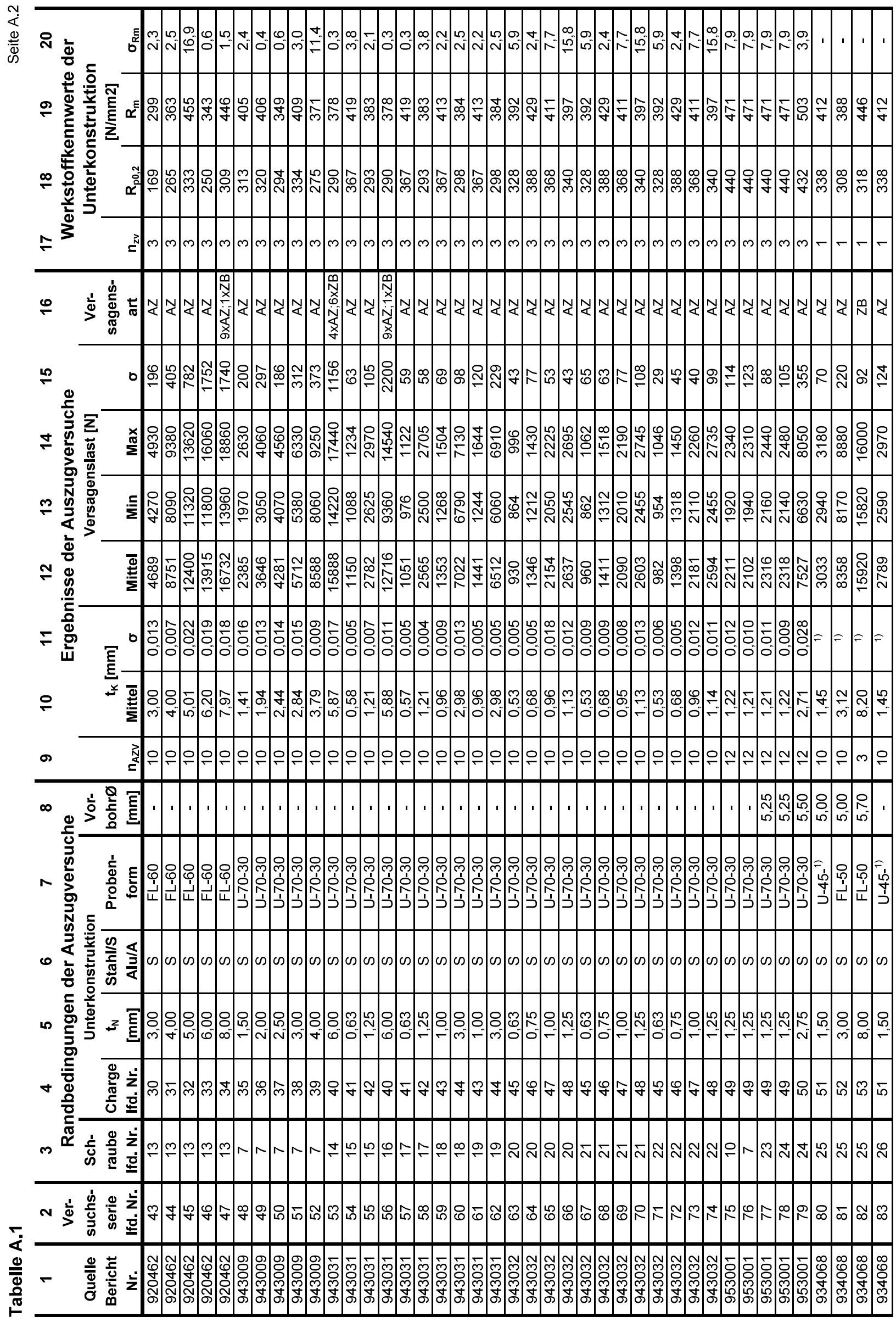




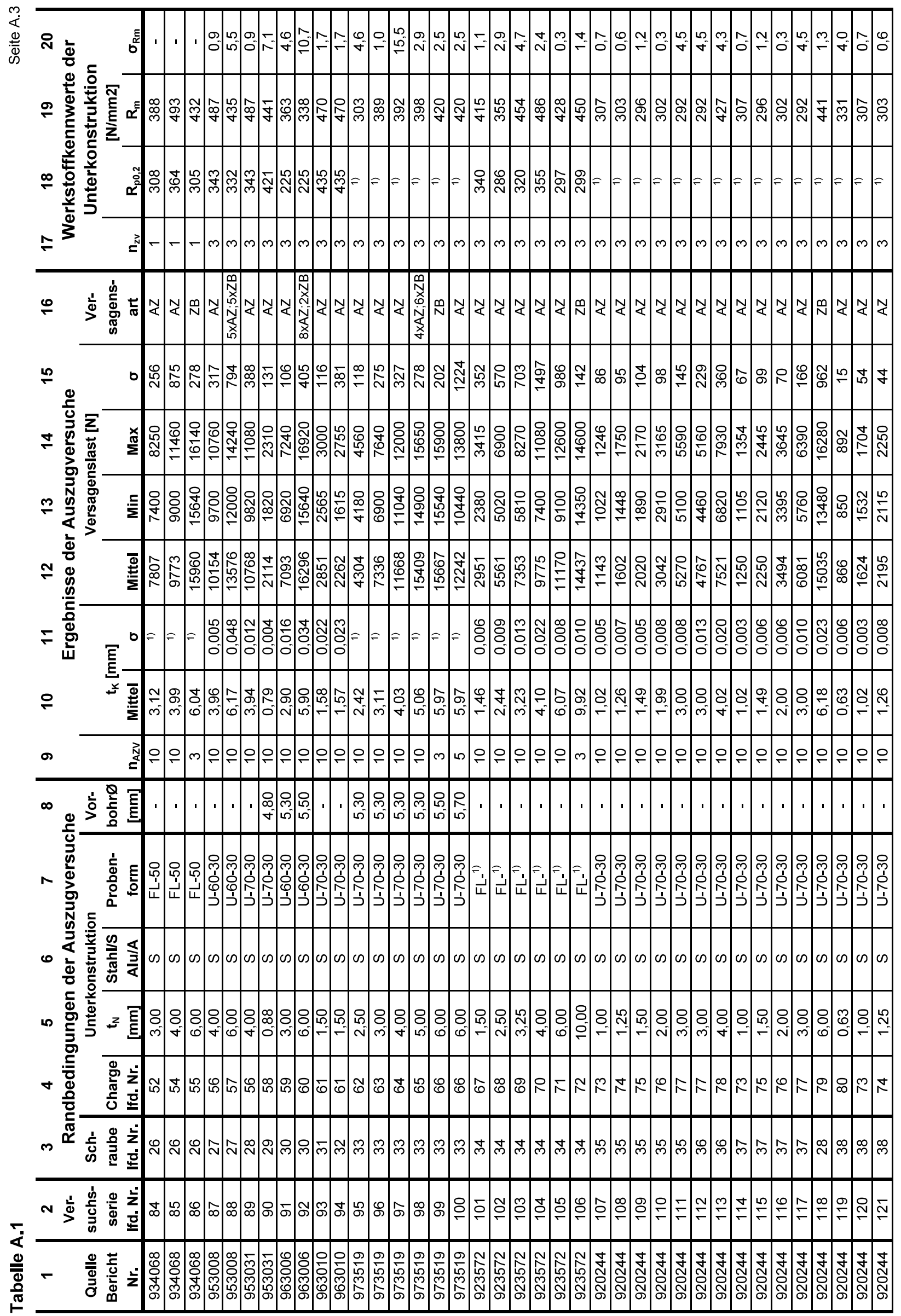




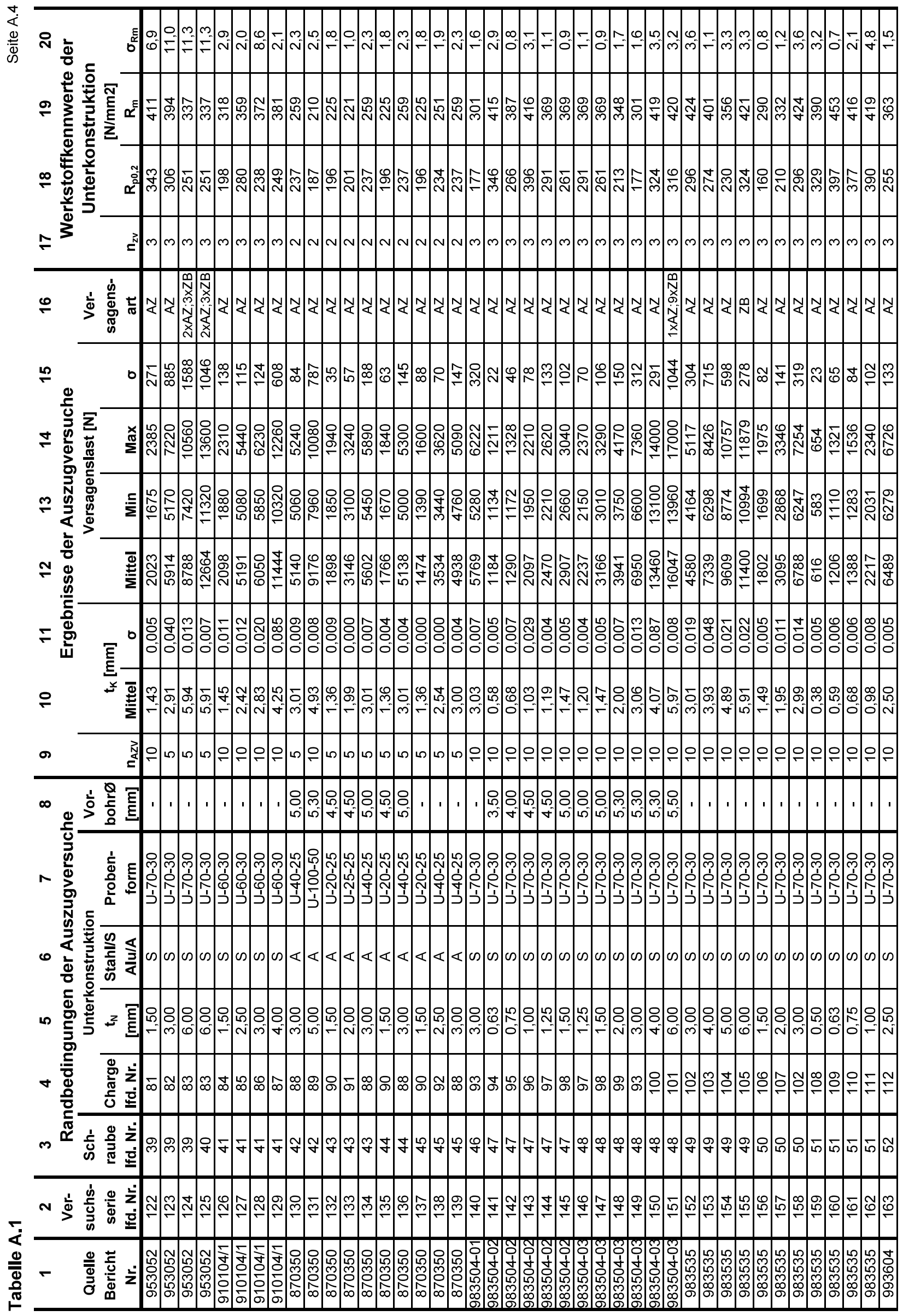




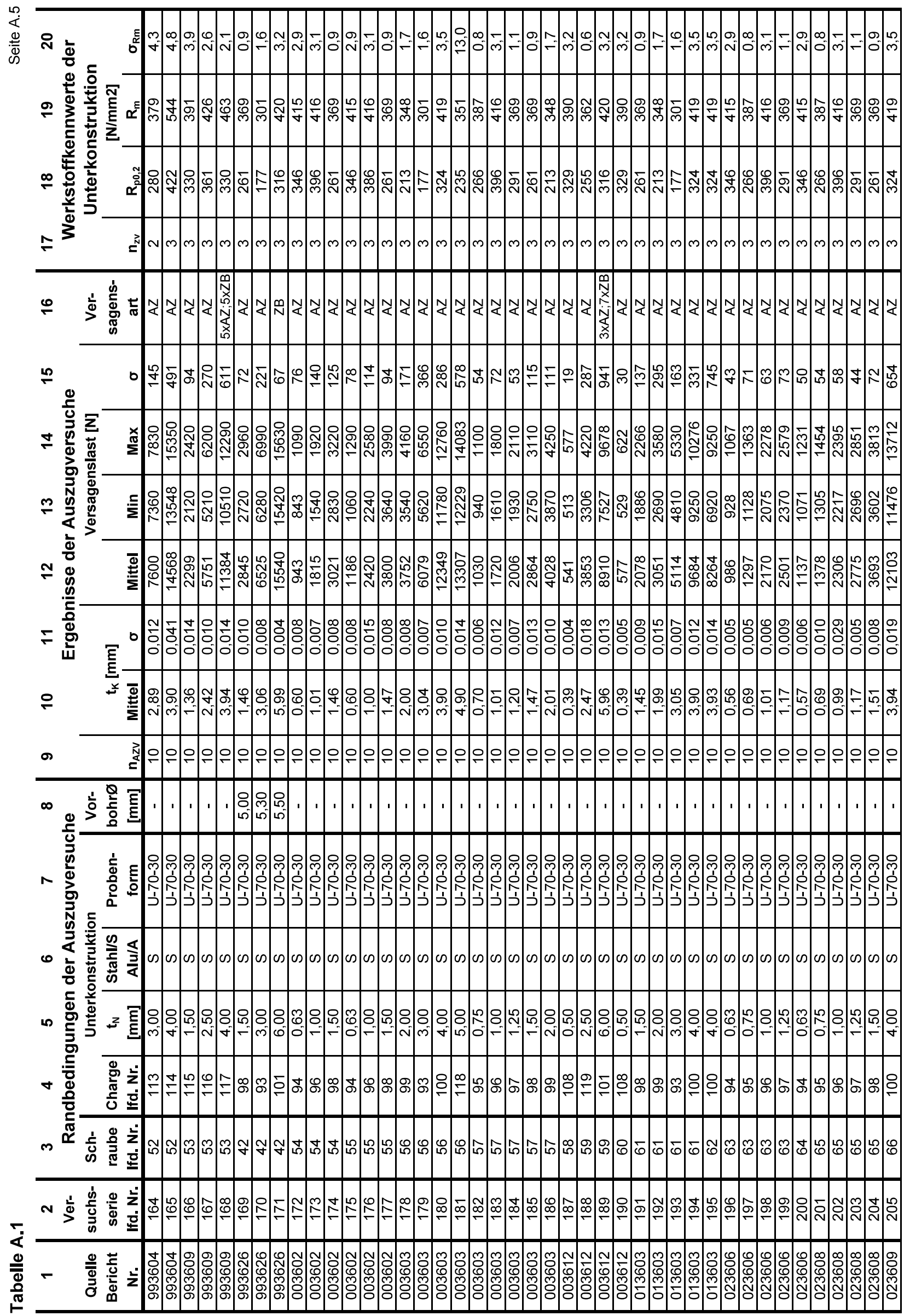




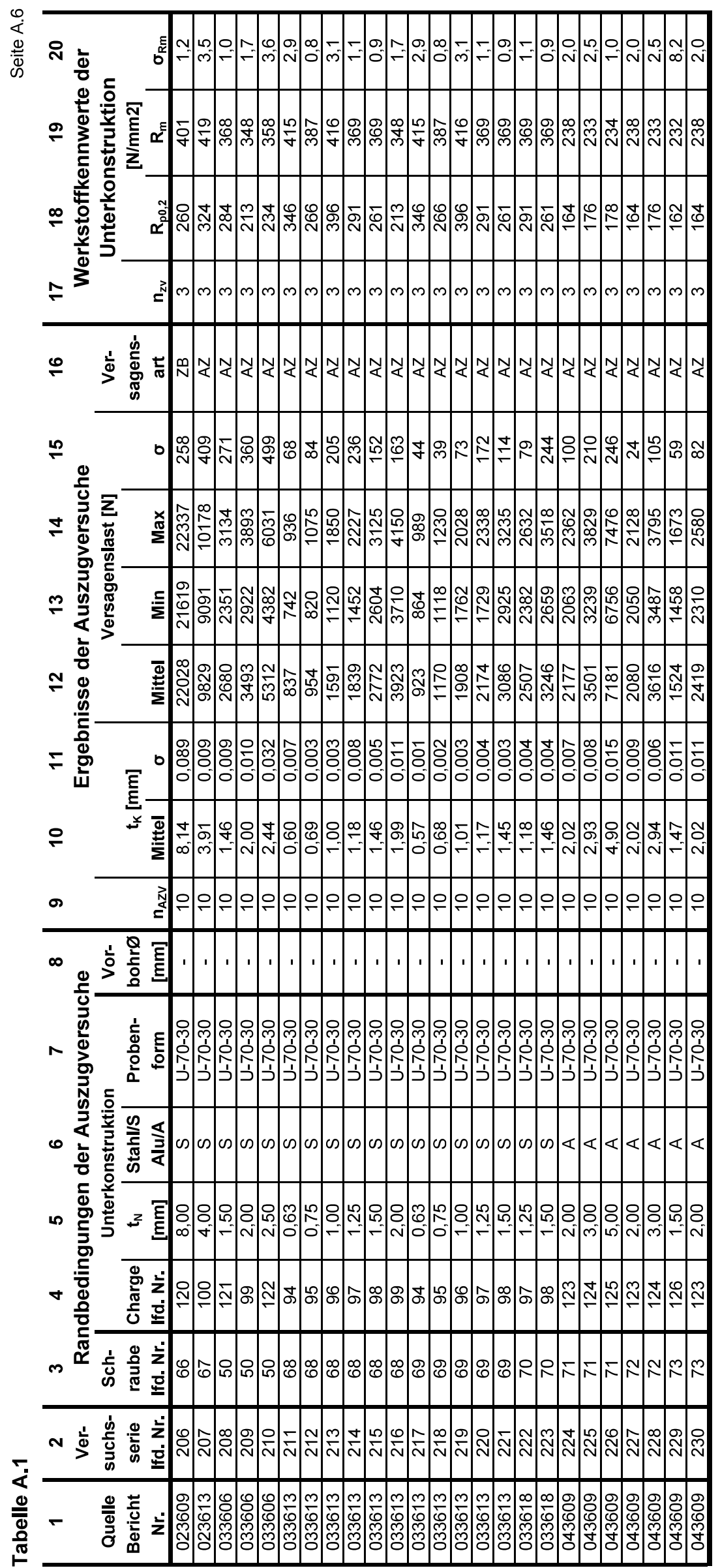

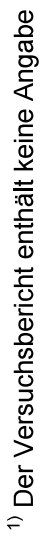




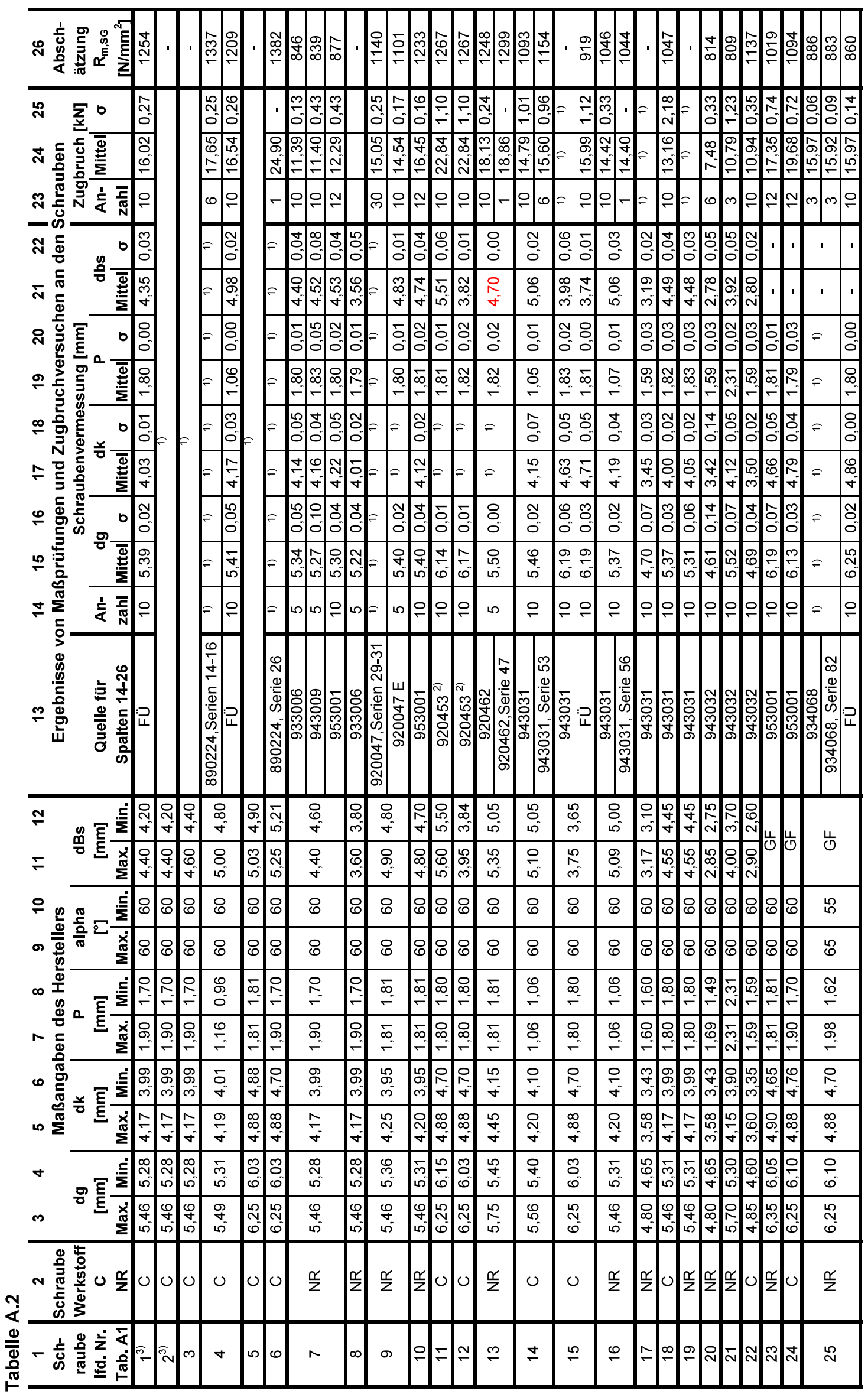




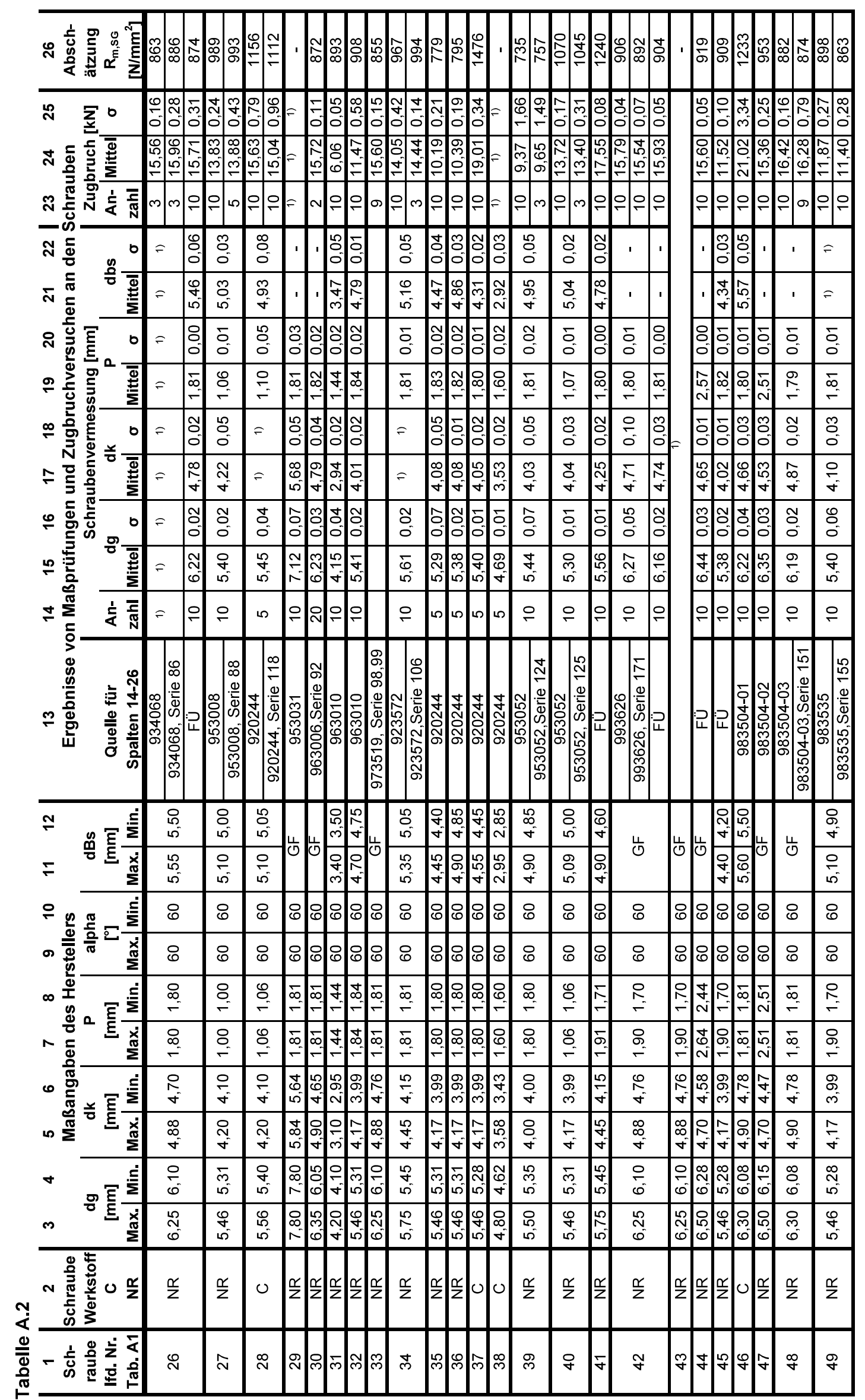




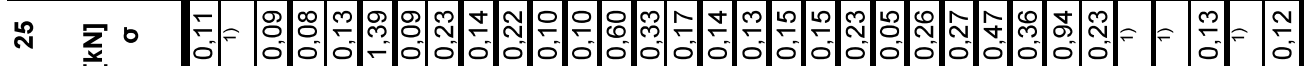

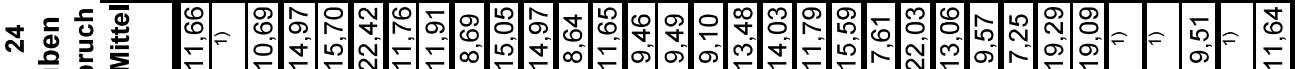

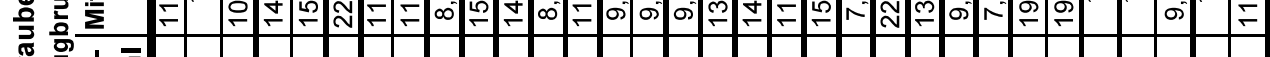

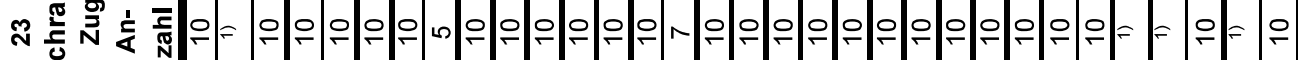

ส

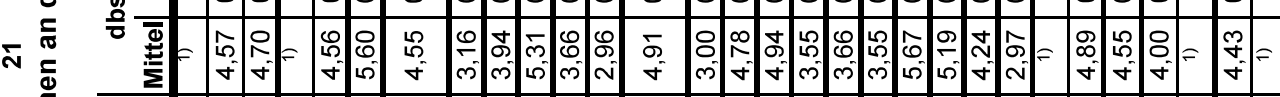

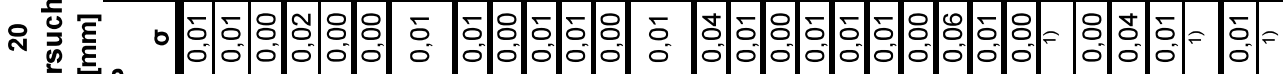

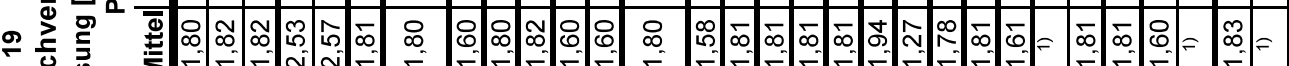

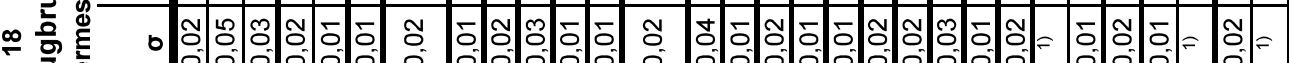

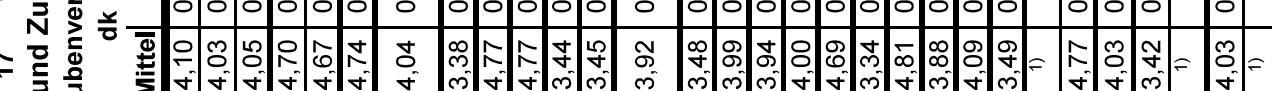

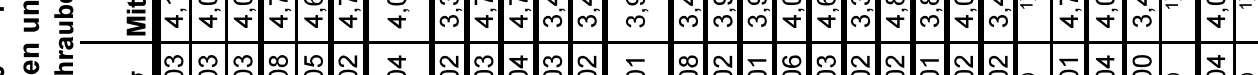

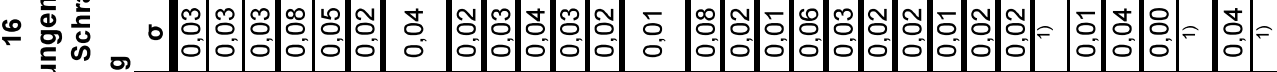

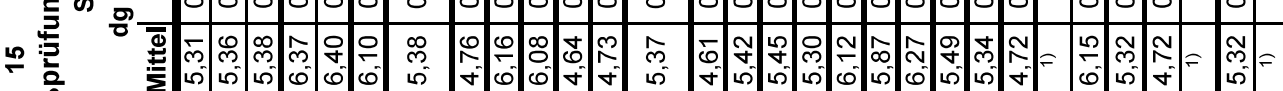

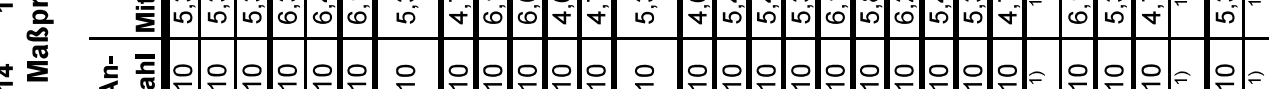

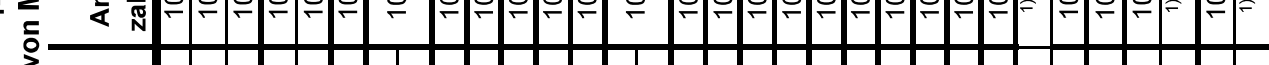

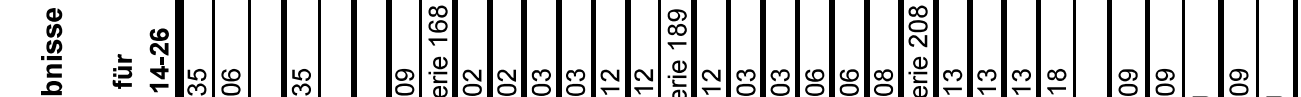

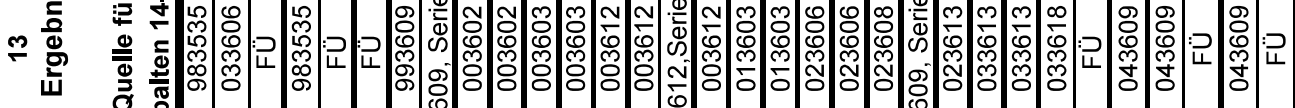

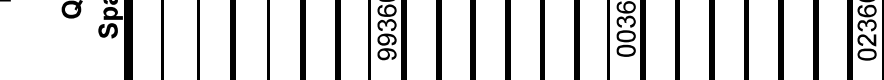

\begin{tabular}{|c|c|c|c|c|c|c|c|c|c|c|c|c|c|c|c|c|c|}
\hline$\omega g \bar{\varepsilon}$ & $f$ & 아 & & 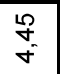 & के & : & 12 & $\begin{array}{l}\infty \\
\infty \\
\infty \\
\forall\end{array}$ & 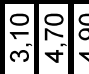 & 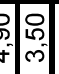 & 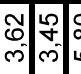 & $\begin{array}{l}0 \\
0 \\
6\end{array}$ & 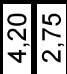 & 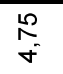 & $\begin{array}{l}8 \\
8 \\
\forall\end{array}$ & 8 & $\underset{+}{+}$ \\
\hline 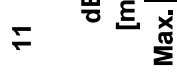 & ○ & 8 & & 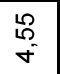 & जे & $\begin{array}{l}3 \\
0 \\
5\end{array}$ & ని & $\underset{8}{8}$ & 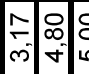 & వ్రి| & 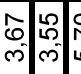 & 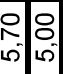 & 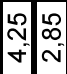 & $\begin{array}{l}\stackrel{R}{R} \\
\&\end{array}$ & $\begin{array}{ll}8 \\
0 \\
\forall \\
\forall \\
\end{array}$ & S. & 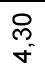 \\
\hline & 0 & 8 & 8 & 8 & ৪) & ৪ & 8 & 8 & :৪্ভ: & : & ৪): & ৪) & $: 8$ & 8 & : & 8 & 8 \\
\hline & 8 & ¿ & 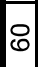 & 8 & ৪) & 8 & 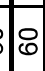 & 8 & \& is & ㅇ. & 이잉 & ৪)ঃ & ৪) & 8 & ৪) & 8 & 8 \\
\hline & $?$ & 莳 & $\infty$ & $\stackrel{\infty}{\stackrel{\infty}{\leftarrow}}$ & $:$ & $\mid$ & $\because$ & $\begin{array}{l}\infty \\
\stackrel{\infty}{-}\end{array}$ & & tit & 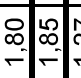 & 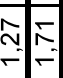 & : & $\stackrel{\Sigma}{\Sigma}$ & $\mid$ & $\stackrel{-}{8}$ & $\stackrel{\infty}{=}$ \\
\hline & ৪ & 志 & $\infty$ & $\stackrel{\infty}{\stackrel{\infty}{\leftarrow}}$ & $:$ & 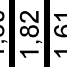 & $\because$ & $\stackrel{\infty}{\stackrel{\infty}{\circ}}$ & 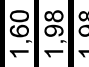 & 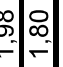 & : & 고요 & 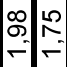 & $\stackrel{s}{\sigma}$ & $\begin{array}{c}0 \\
\vdots \\
-1\end{array}$ & $\stackrel{\circ}{\circ}$ & 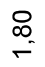 \\
\hline & 8. & 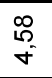 & $\begin{array}{r}R \\
i \\
i\end{array}$ & $\begin{array}{l}\stackrel{g}{g} \\
\dot{m}\end{array}$ & के & $?$ & 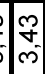 & $\begin{array}{l}\mathscr{P} \\
\dot{m} \\
\dot{m}\end{array}$ & 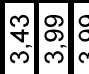 & ग্পি| & 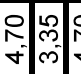 & 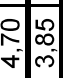 & 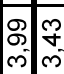 & $\begin{array}{l}0 \\
\stackrel{9}{*} \\
\forall\end{array}$ & \begin{tabular}{|l|} 
\\
\\
p.
\end{tabular} & 荘 & g \\
\hline & $\underset{f}{7}$ & $\begin{array}{l}0 \\
+ \\
+\end{array}$ & $\begin{array}{l}\infty \\
\infty \\
\infty \\
f \\
-1\end{array}$ & $\stackrel{F}{F}$ & 贯: & 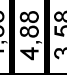 & . & $\stackrel{f}{f}$ & 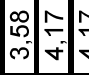 & $=$ & 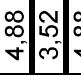 & 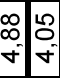 & 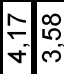 & $\begin{array}{l}\infty \\
\infty \\
\vdots \\
\forall\end{array}$ & $\begin{array}{ll}7 \\
7 \\
\end{array}$ & 恶 & $\stackrel{5}{f}$ \\
\hline$ه$ 口 & $\stackrel{\infty}{N}$ & 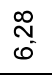 & 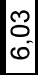 & 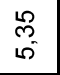 & 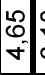 & $\begin{array}{c}0 \\
0 \\
0\end{array}$ & $\underset{\forall}{\pi}$ & $\bar{n}$ & 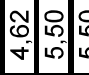 & 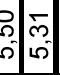 & \begin{tabular}{lll}
0 & 0 \\
\hdashline \\
0
\end{tabular} & 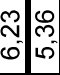 & - & $\frac{\circ}{6}$ & 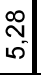 & $\tilde{\sigma}$ & 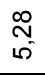 \\
\hline 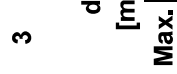 & $\begin{array}{l}9 \\
q \\
6\end{array}$ & 0 & & $\begin{array}{l}0 \\
0 \\
\omega_{0}\end{array}$ & 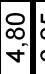 & 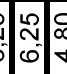 & $\begin{array}{l}\infty \\
\infty \\
\forall \\
\forall\end{array}$ & $\begin{array}{l}9 \\
9 \\
0 \\
0\end{array}$ & 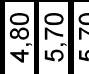 & \begin{tabular}{|l|l|}
0 \\
0
\end{tabular} & 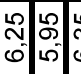 & $\begin{array}{lll}0 & 0 \\
0 & 0 \\
0 & 5 \\
\end{array}$ & 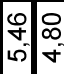 & 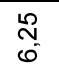 & \begin{tabular}{c|c|}
6 \\
0 \\
$\omega^{\prime}$
\end{tabular} & $\begin{array}{l}\infty \\
\infty \\
\forall \\
\forall\end{array}$ & $\begin{array}{c}0 \\
0 \\
6\end{array}$ \\
\hline 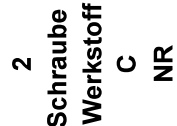 & $\frac{\underline{r}}{z}$ & $\frac{\underline{r}}{z}$ & 0 & $\frac{\underline{x}}{z}$ & $\frac{\mathbf{x}}{\mathrm{z}}$ & $\frac{r}{z} \mid$ & 10 & $\frac{\underline{\alpha}}{z}$ & $\frac{\mu}{z}$ & $\frac{\underline{r}}{z}$ & & $v \mid \frac{\alpha}{z}$ & $\mid \frac{Y}{z}$ & 0 & $\frac{x}{z} \mid$ & $\frac{\underline{v}}{z}$ & $\frac{\underline{r}}{z}$ \\
\hline 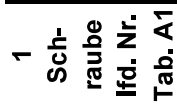 & 8) & in & & దై & & & $\infty$ & 욤 & ৪ & & & & :|৪ & $?$ & $\Sigma$ & N & $\stackrel{2}{2}$ \\
\hline
\end{tabular}

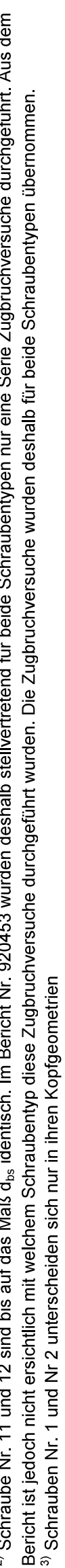




\section{Anhang B}

Werkst offgesetze

für die

FE-Berechnungen 
Seite B.1
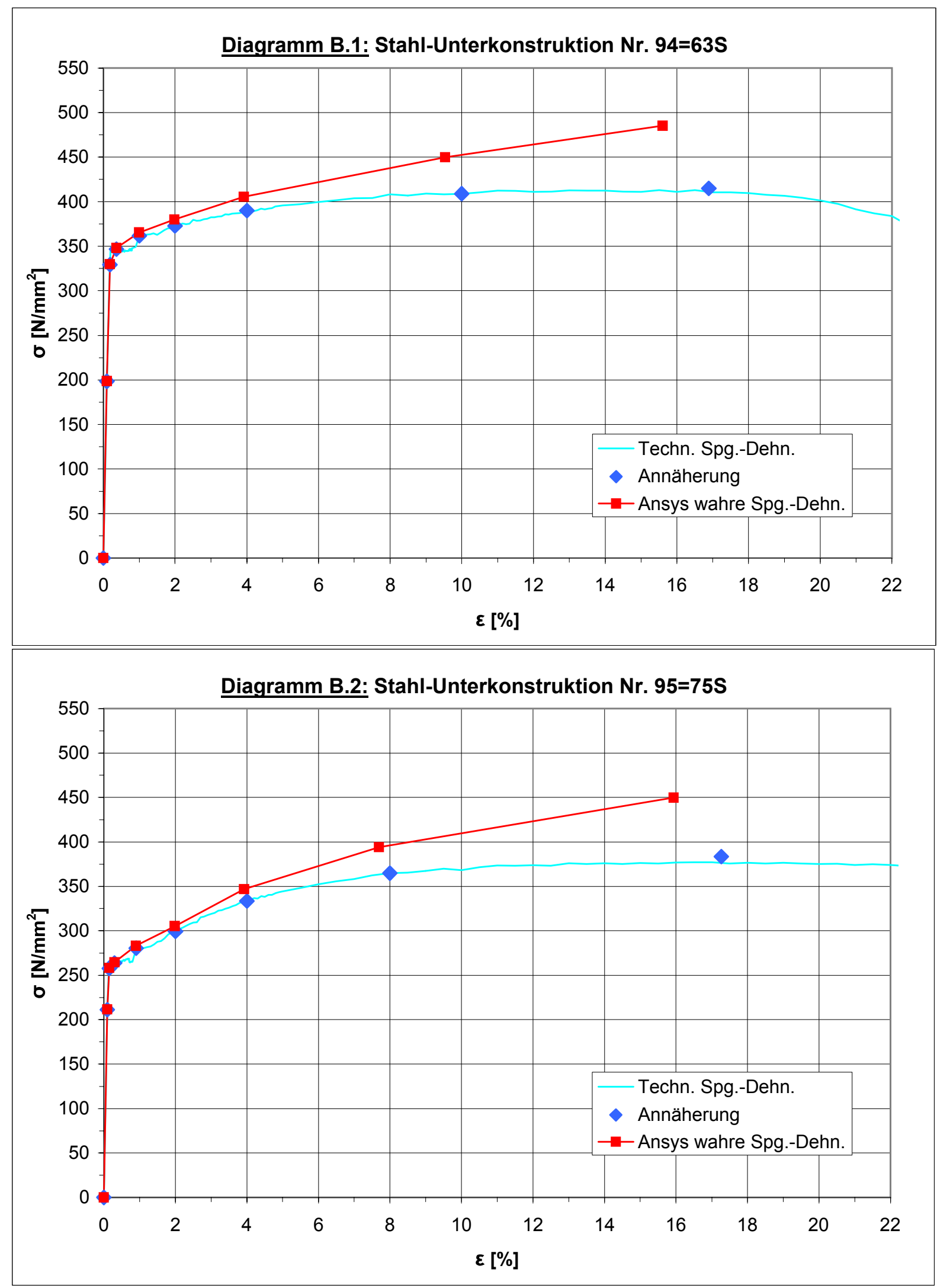
Seite B.2
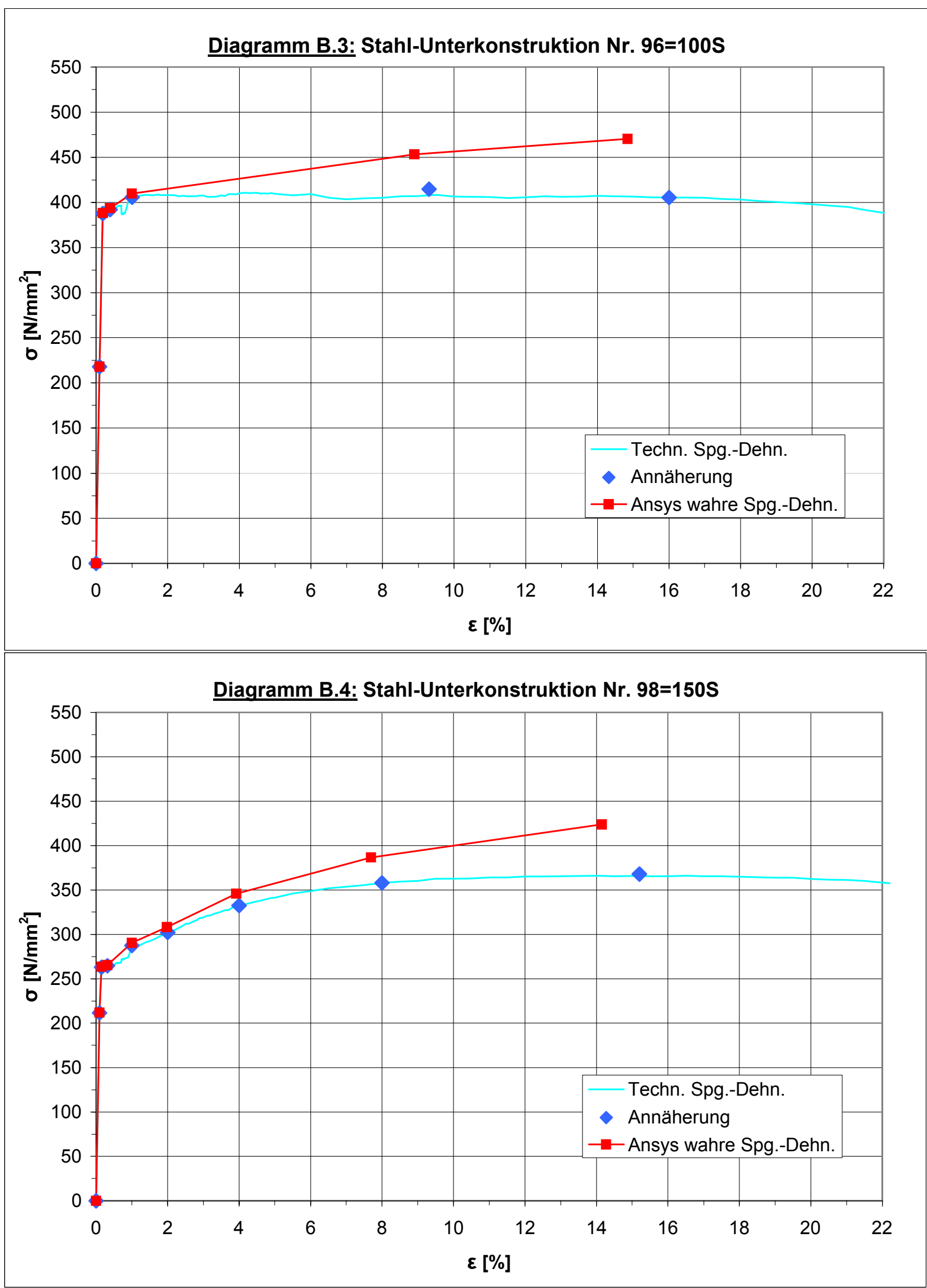
Seite B.3
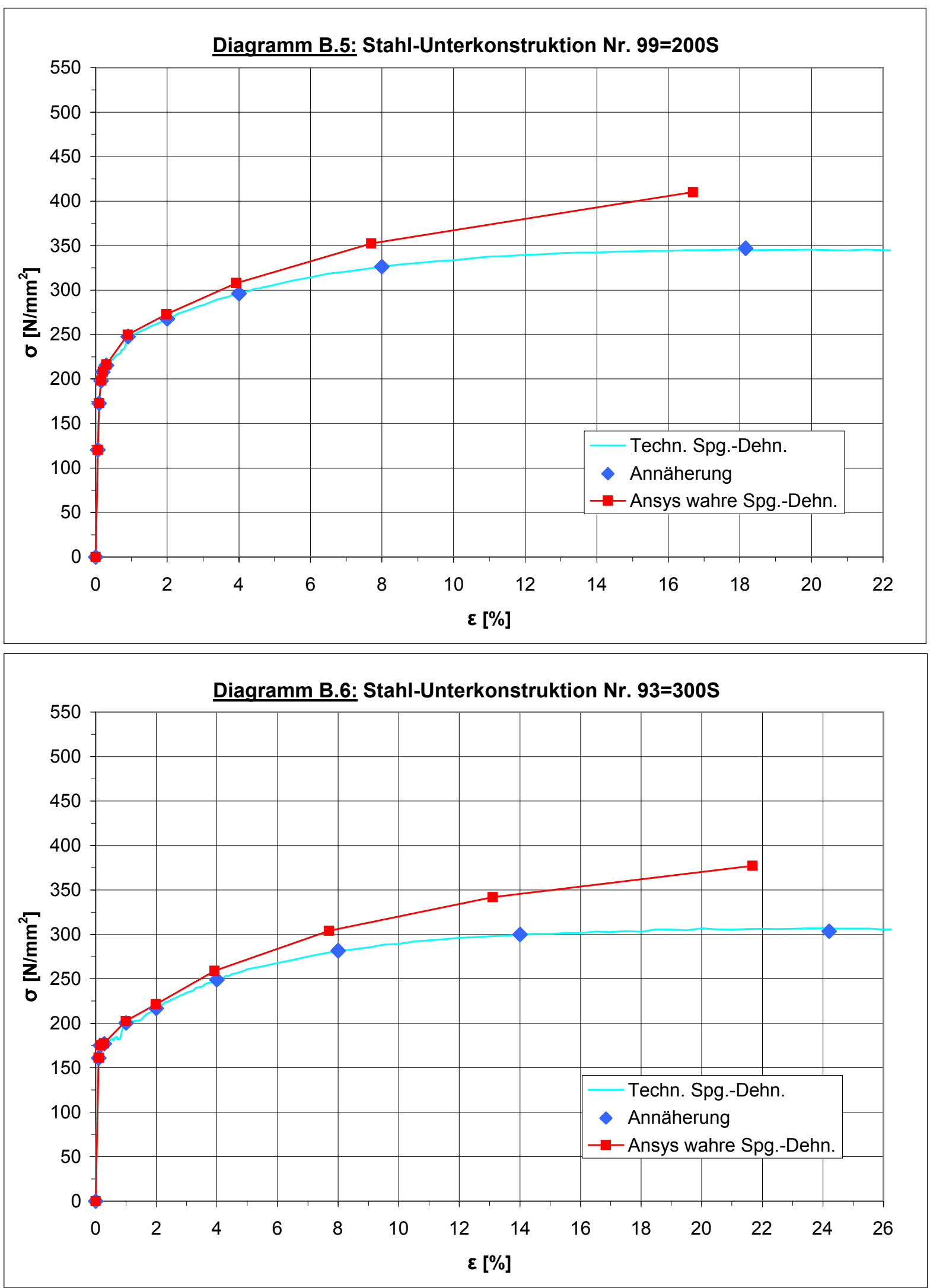
Seite B.4
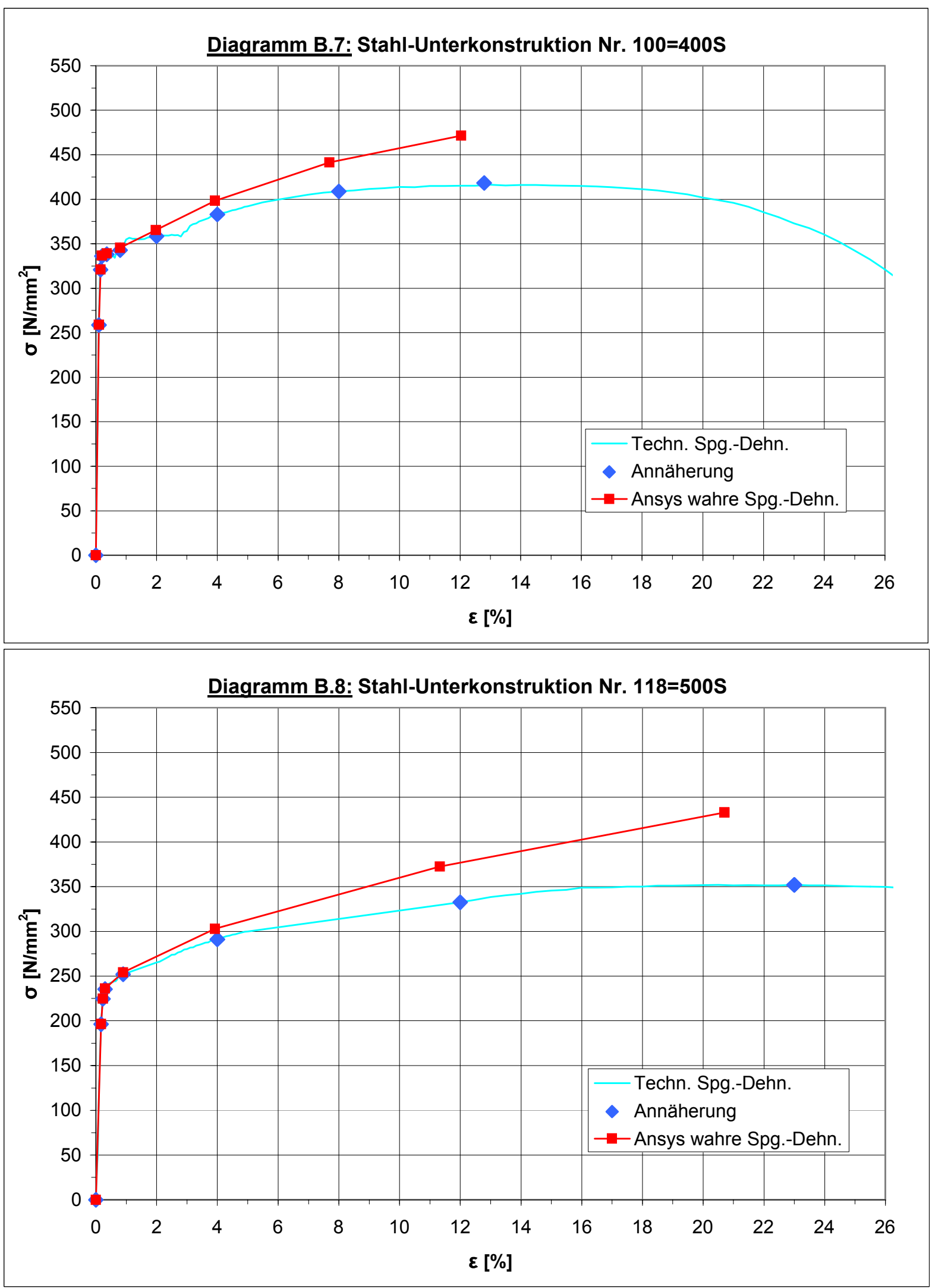
Seite B.5
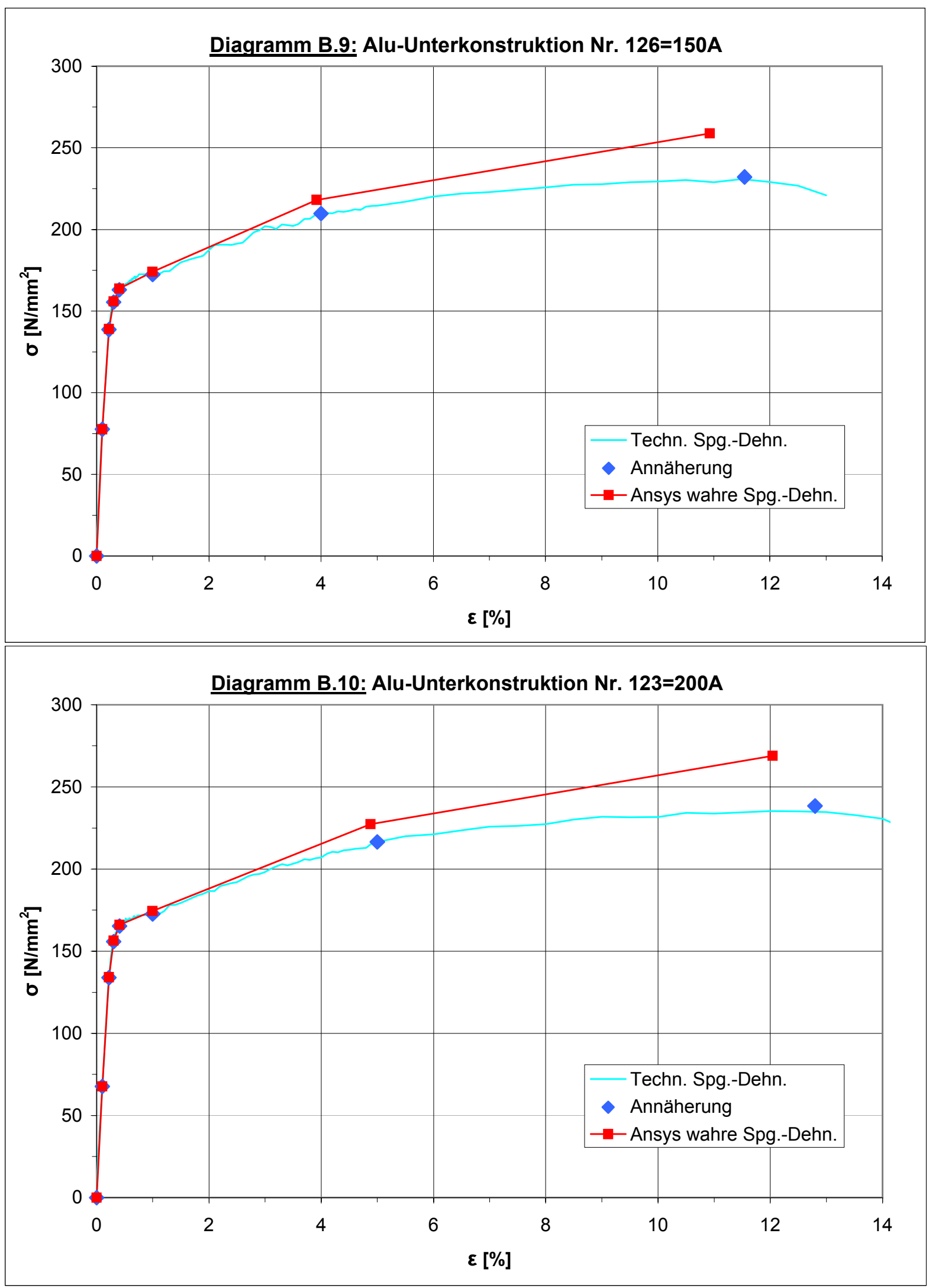
Seite B.6
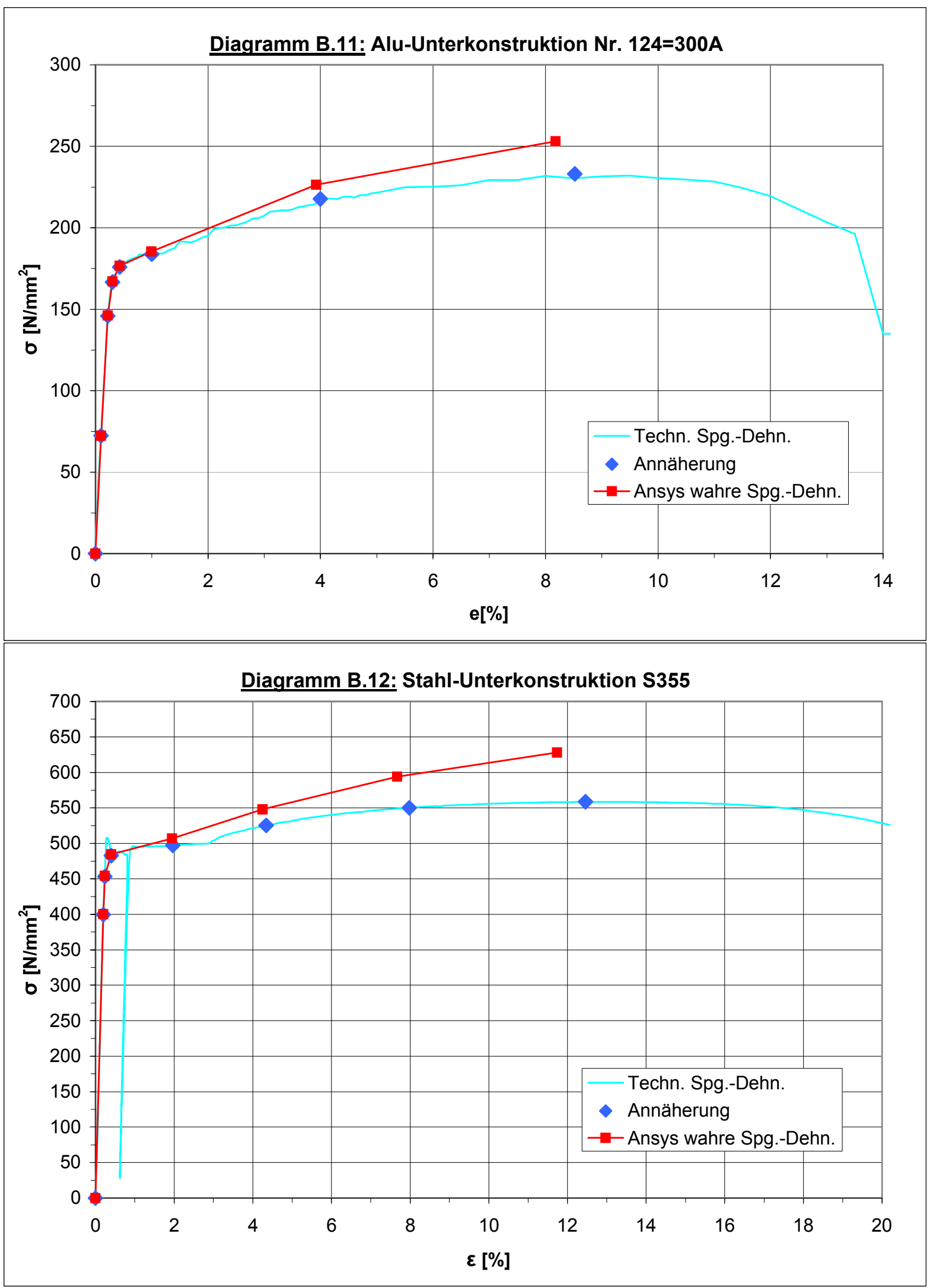

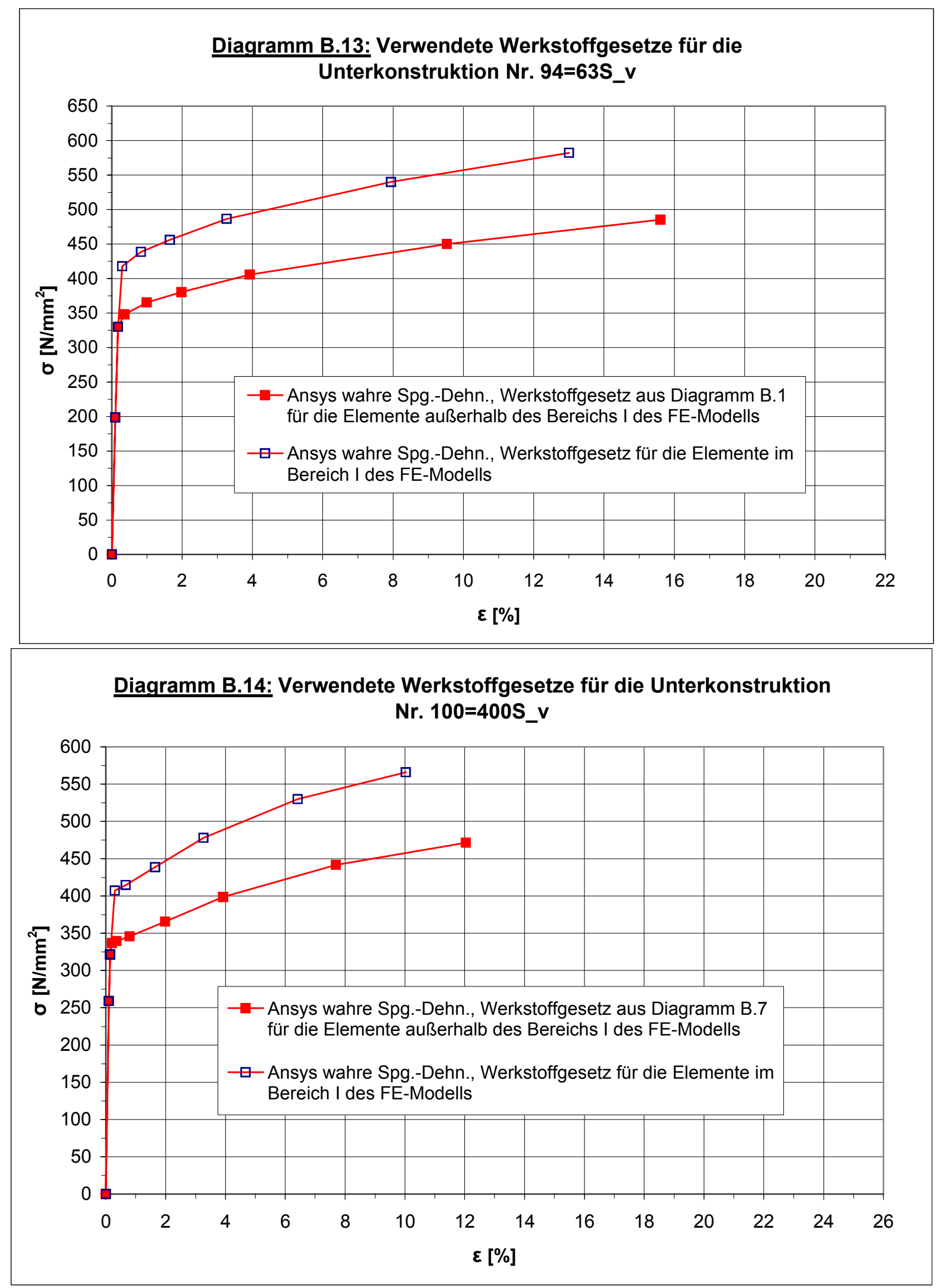
Seite B.8
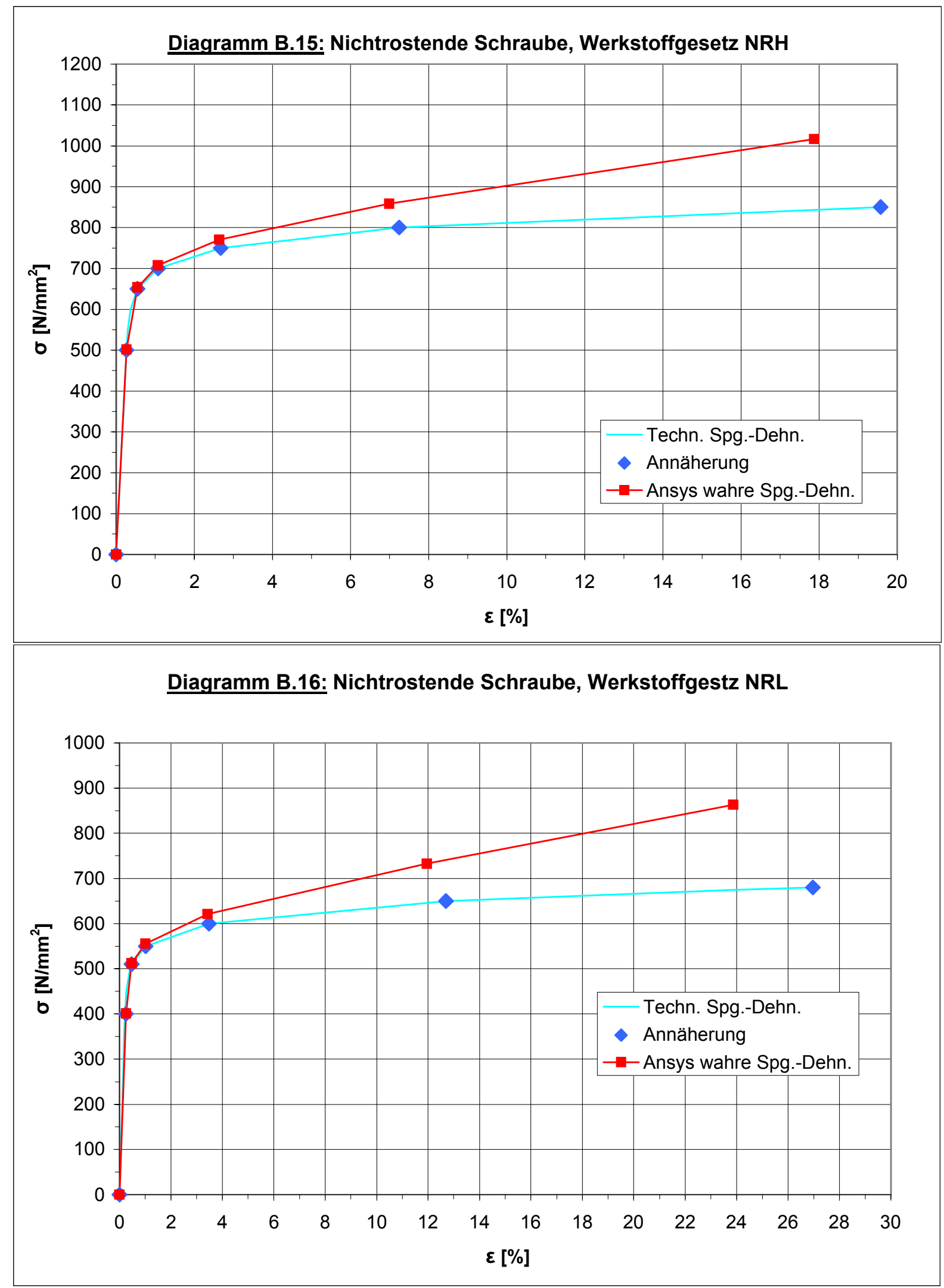
Seite B.9

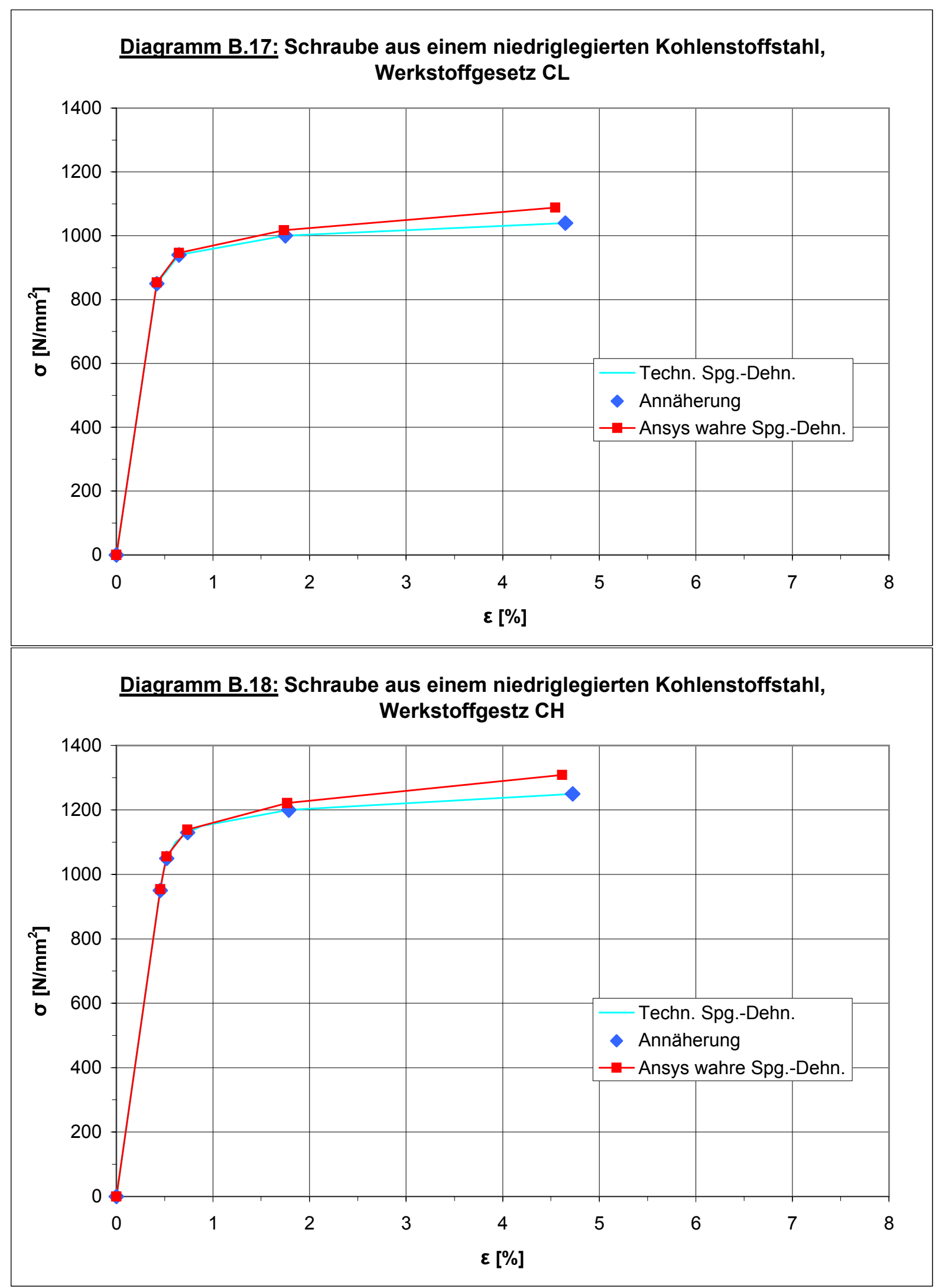


Seite B.10

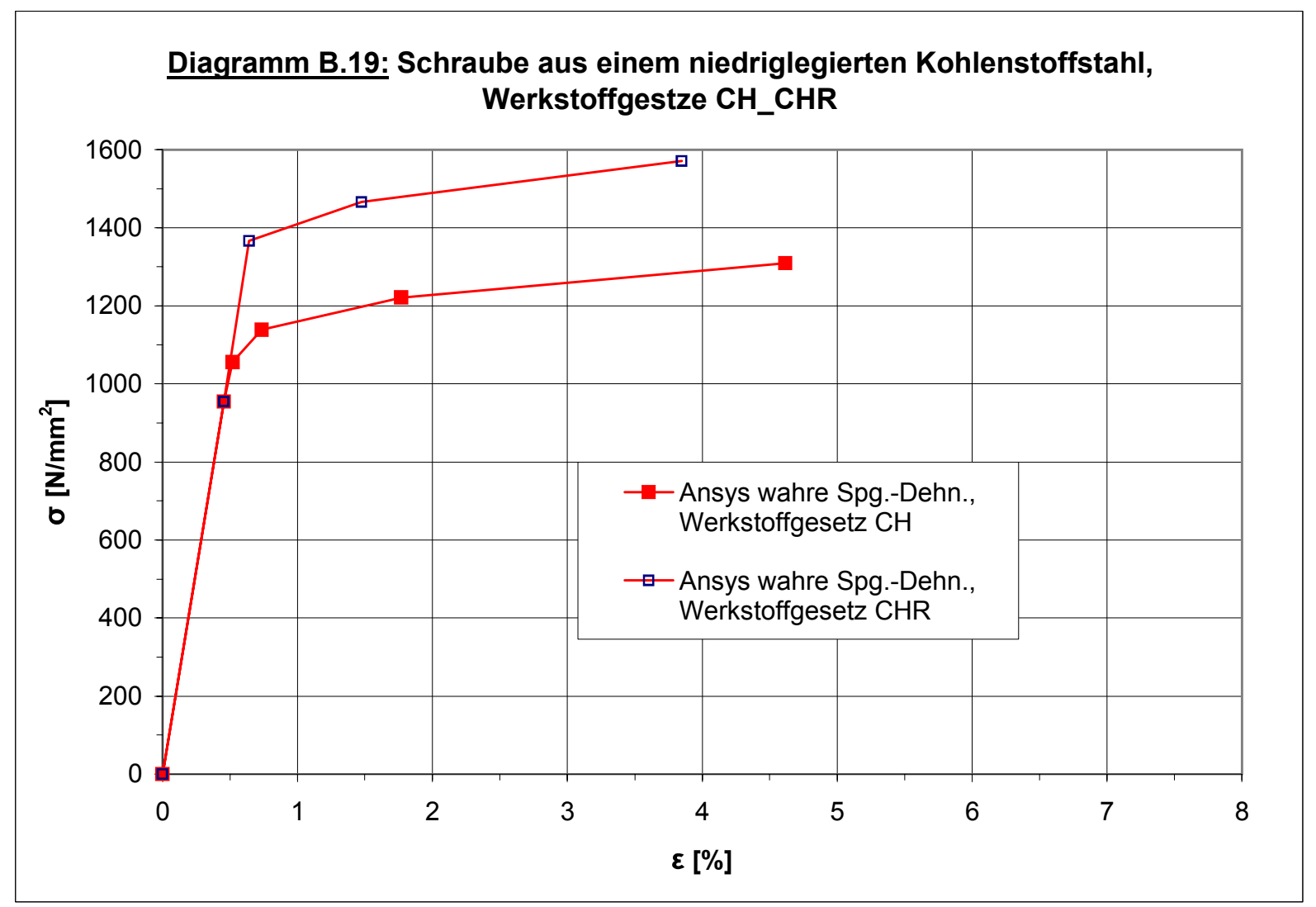




\section{Anhang C}

Vergleich der

Last-Verformungskurven

Versuch/FEM 
Seite C.1
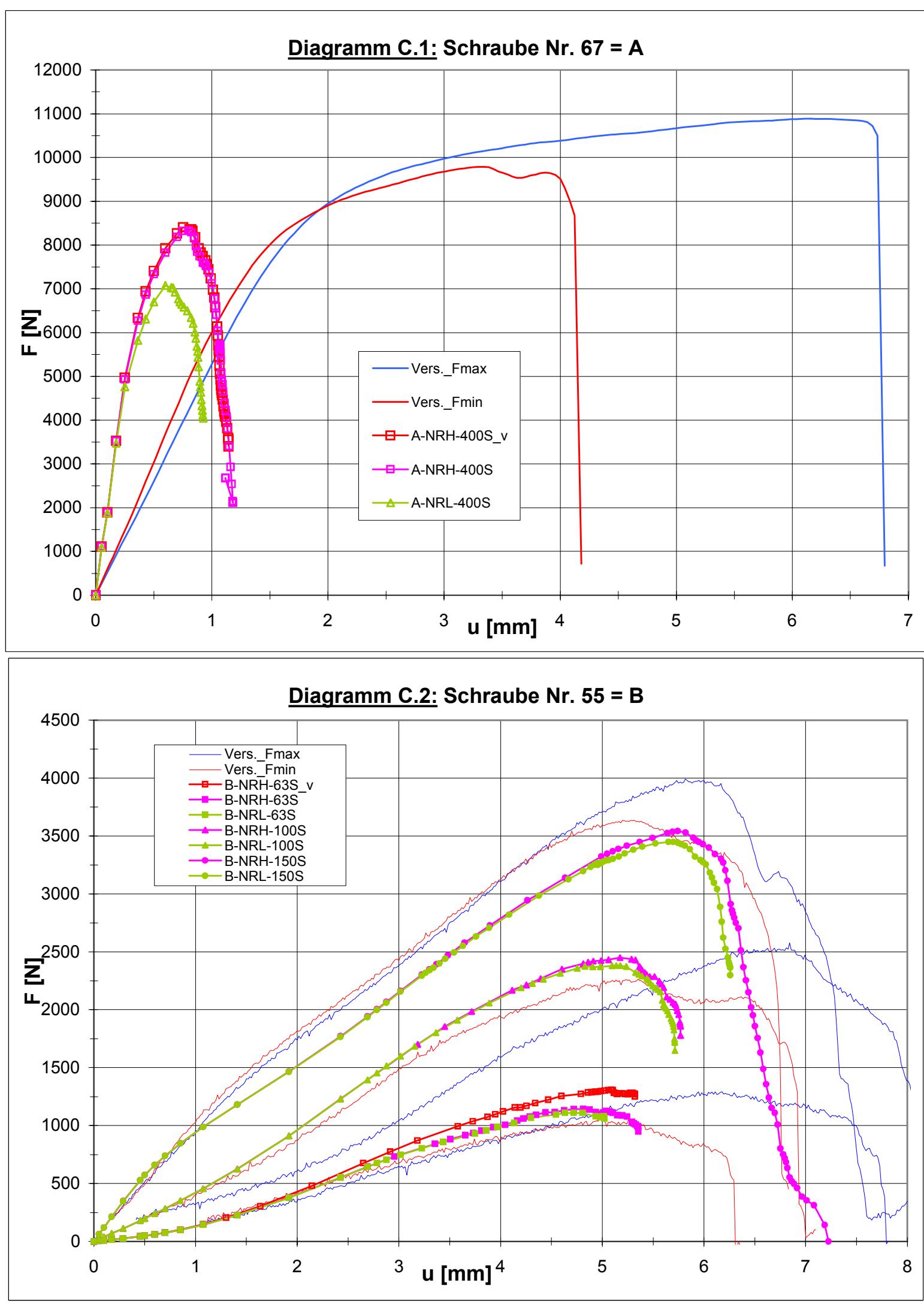
Seite C.2
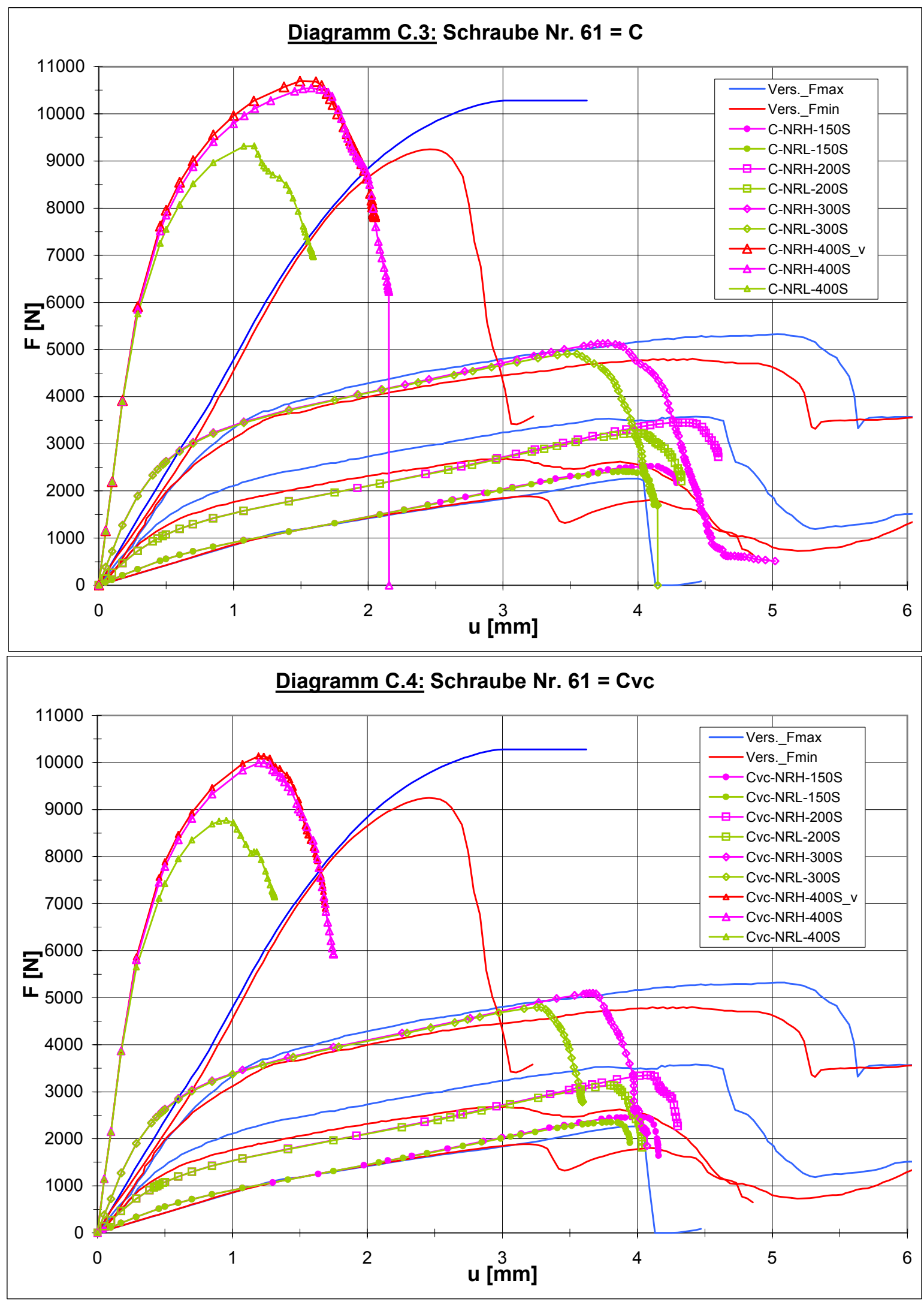
Seite C.3
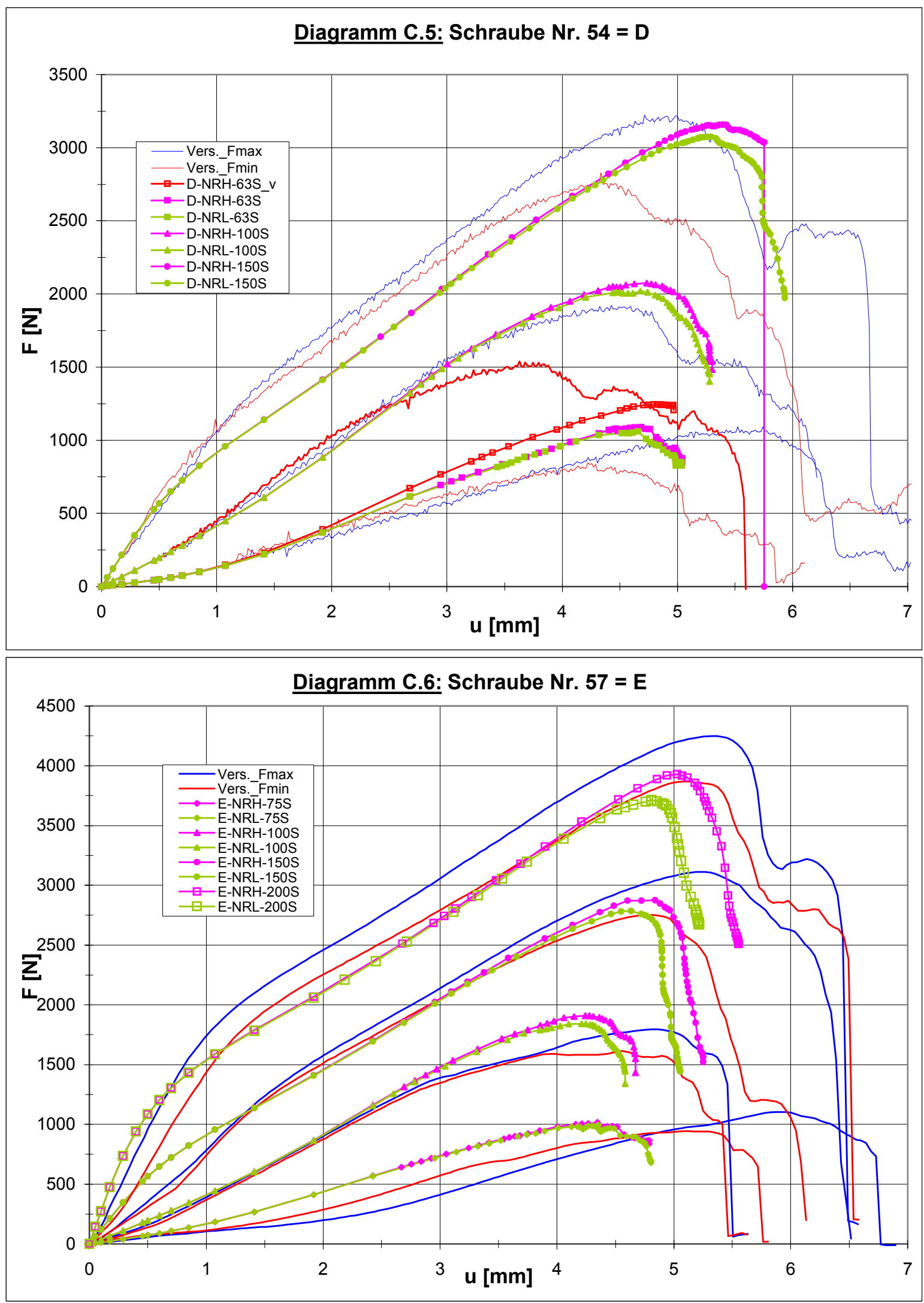
Seite C.4
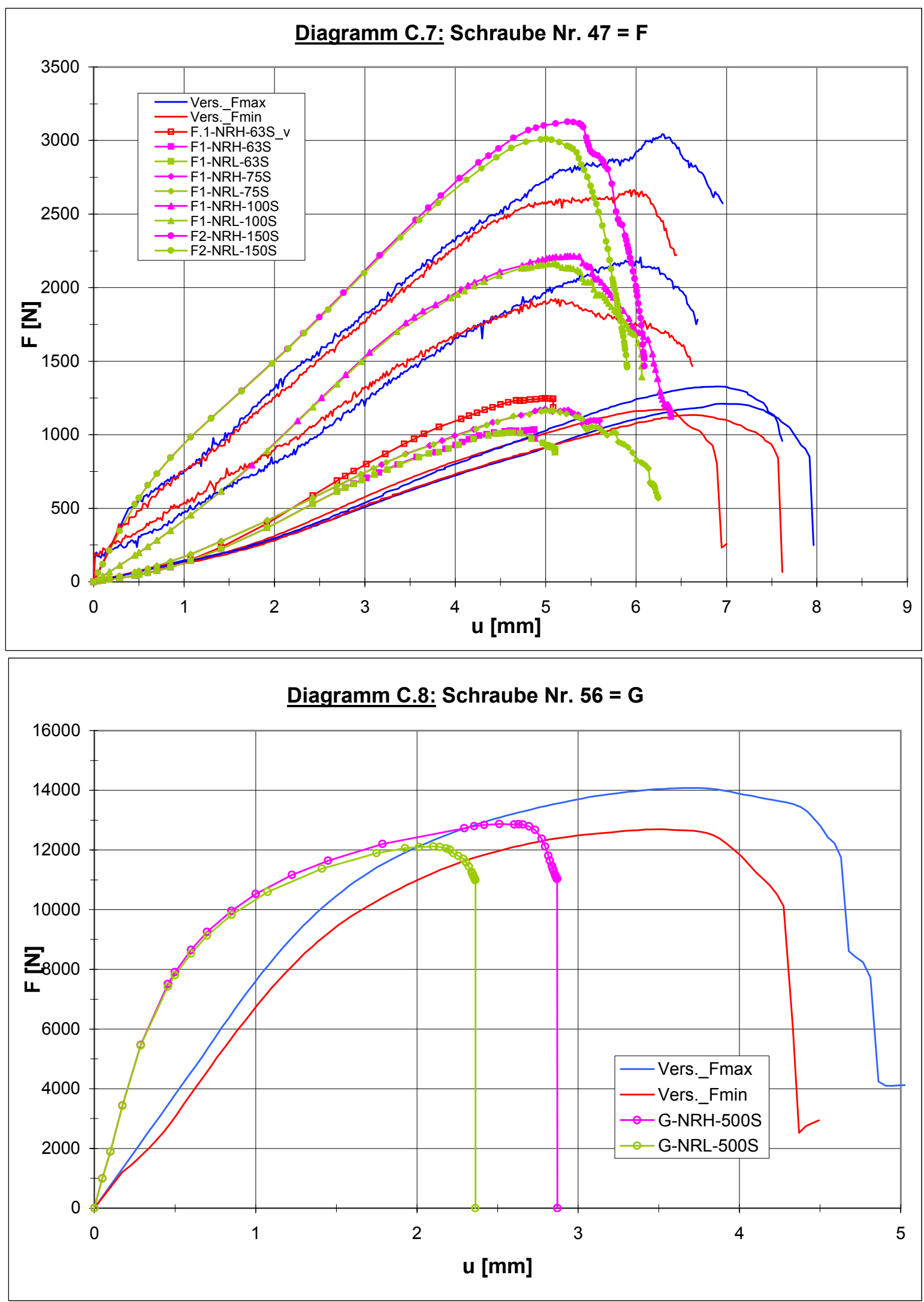
Seite C.5
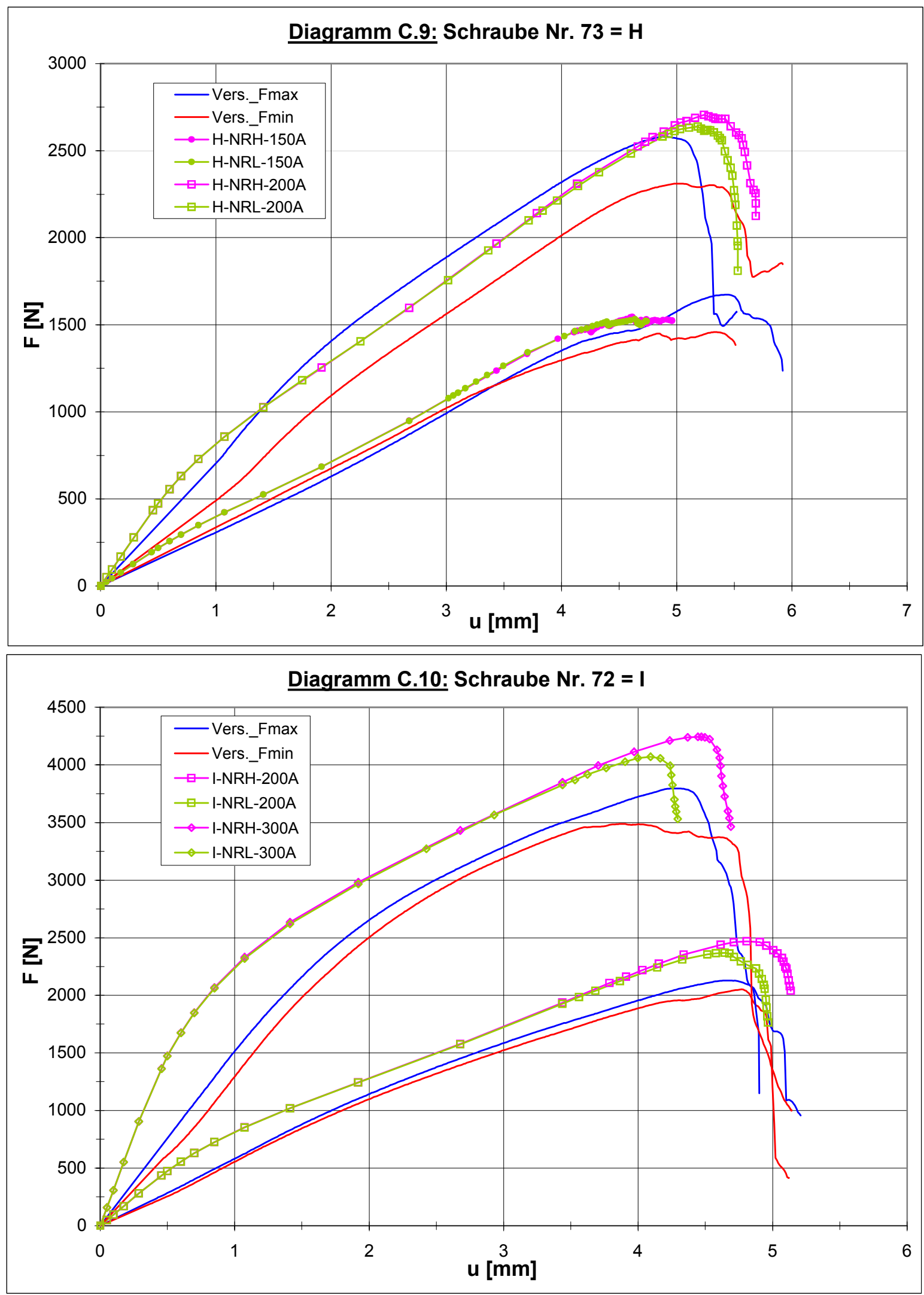
Seite C.6
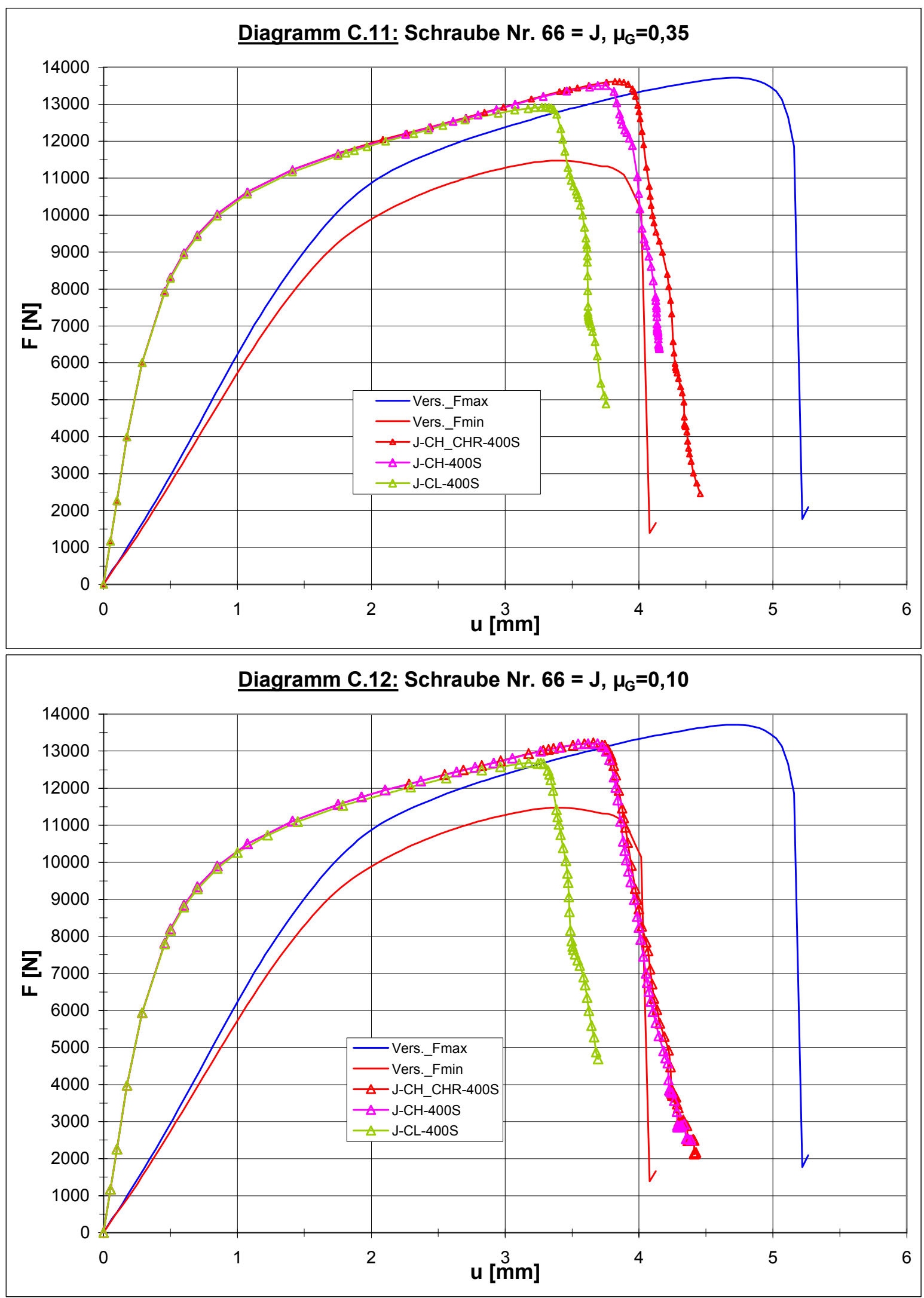


\title{
Anhang D
}

\author{
Ergebnisse der
}

Parameterstudien 

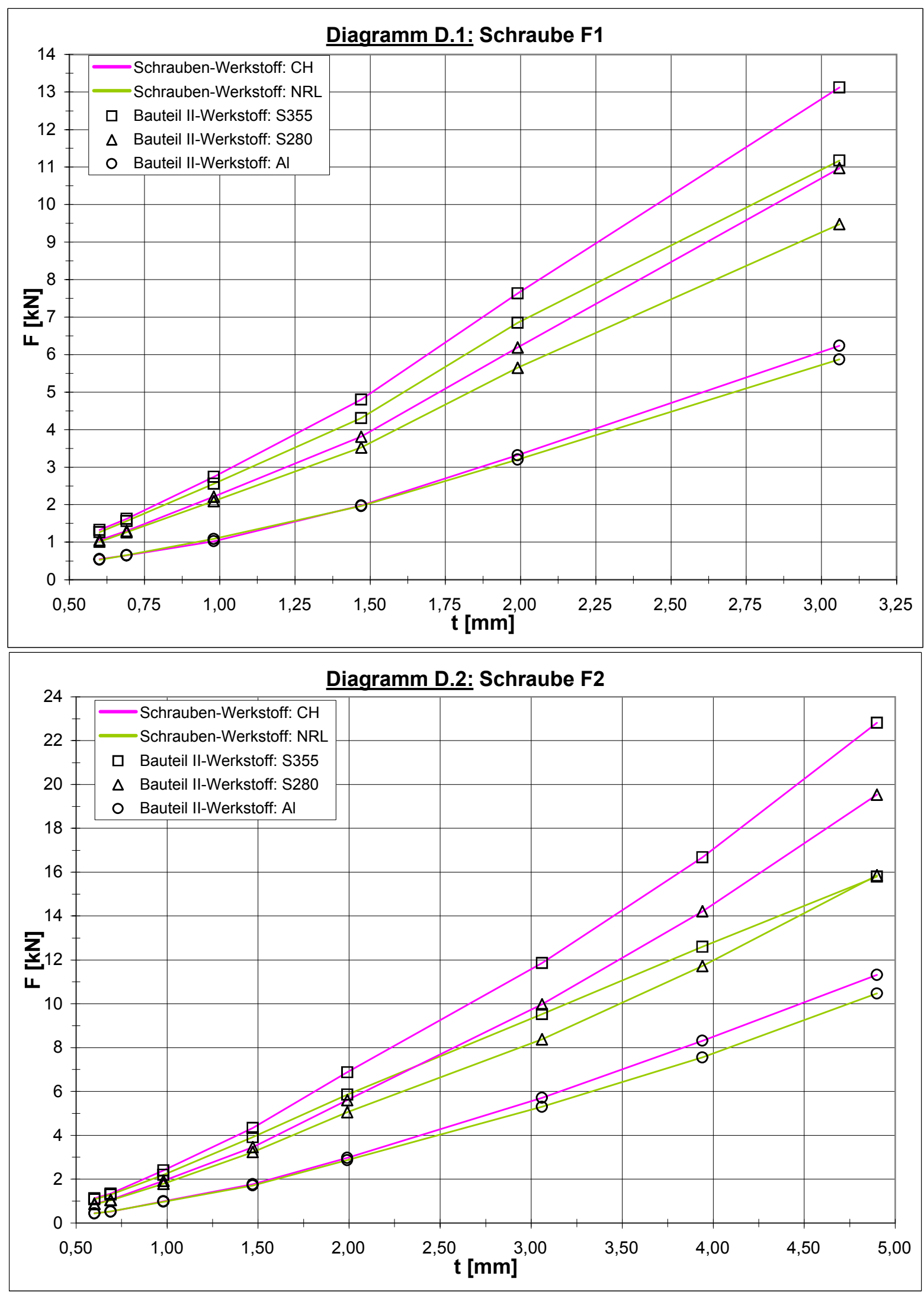
Seite D.2

\section{Diagramm D.3: Schraube B}

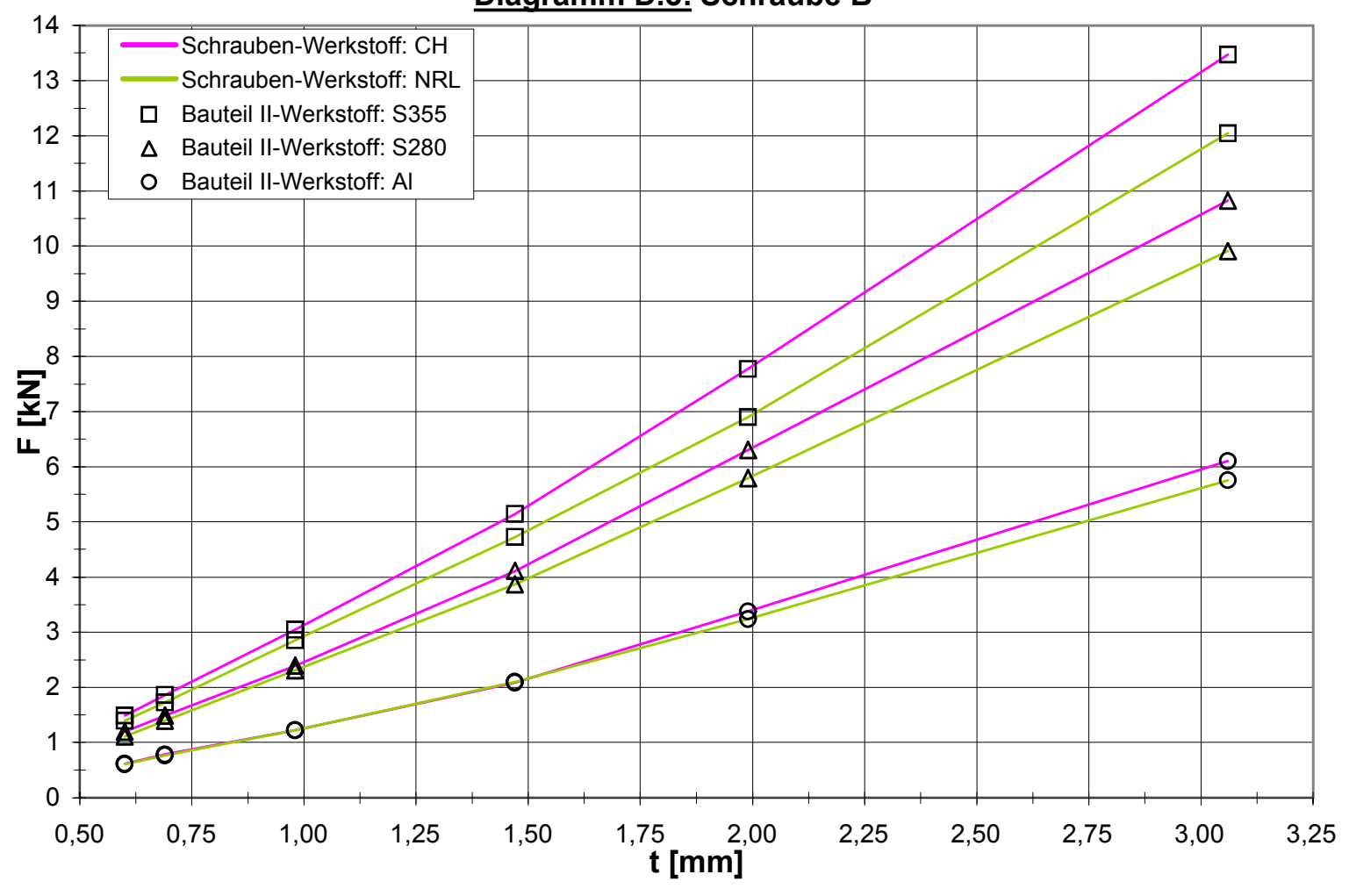

Diagramm D.4: Schraube G

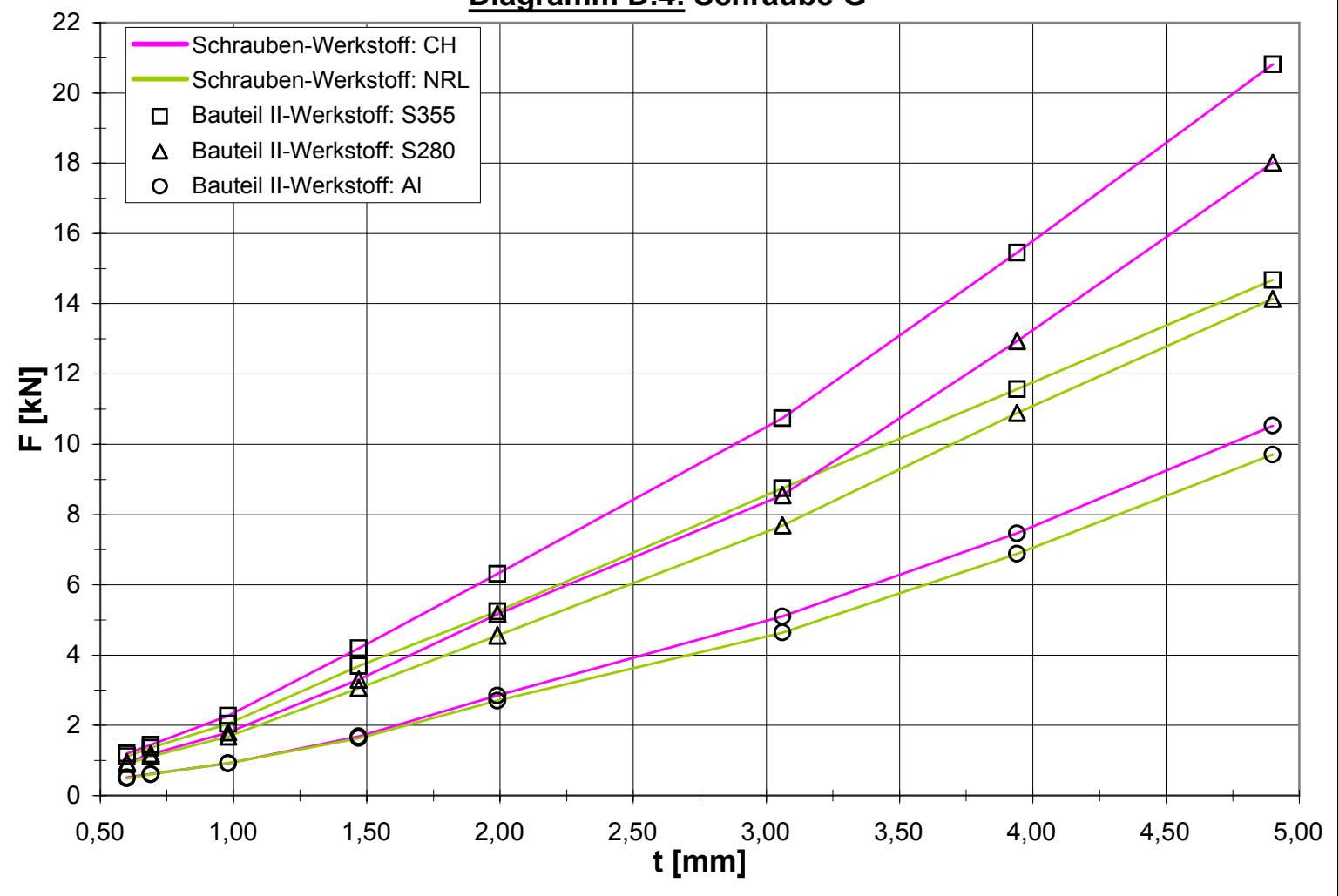


Seite D.3
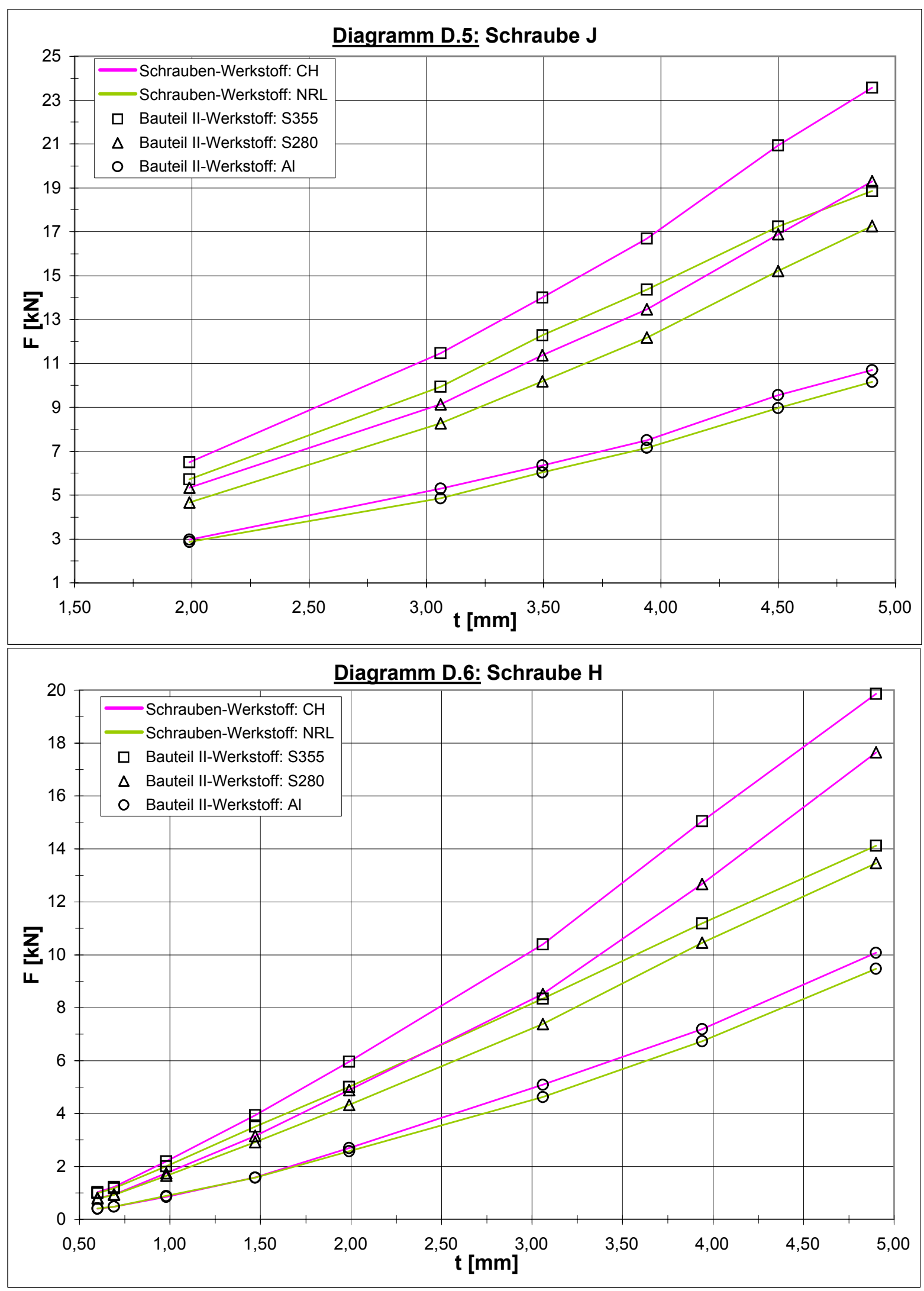
Seite D.4
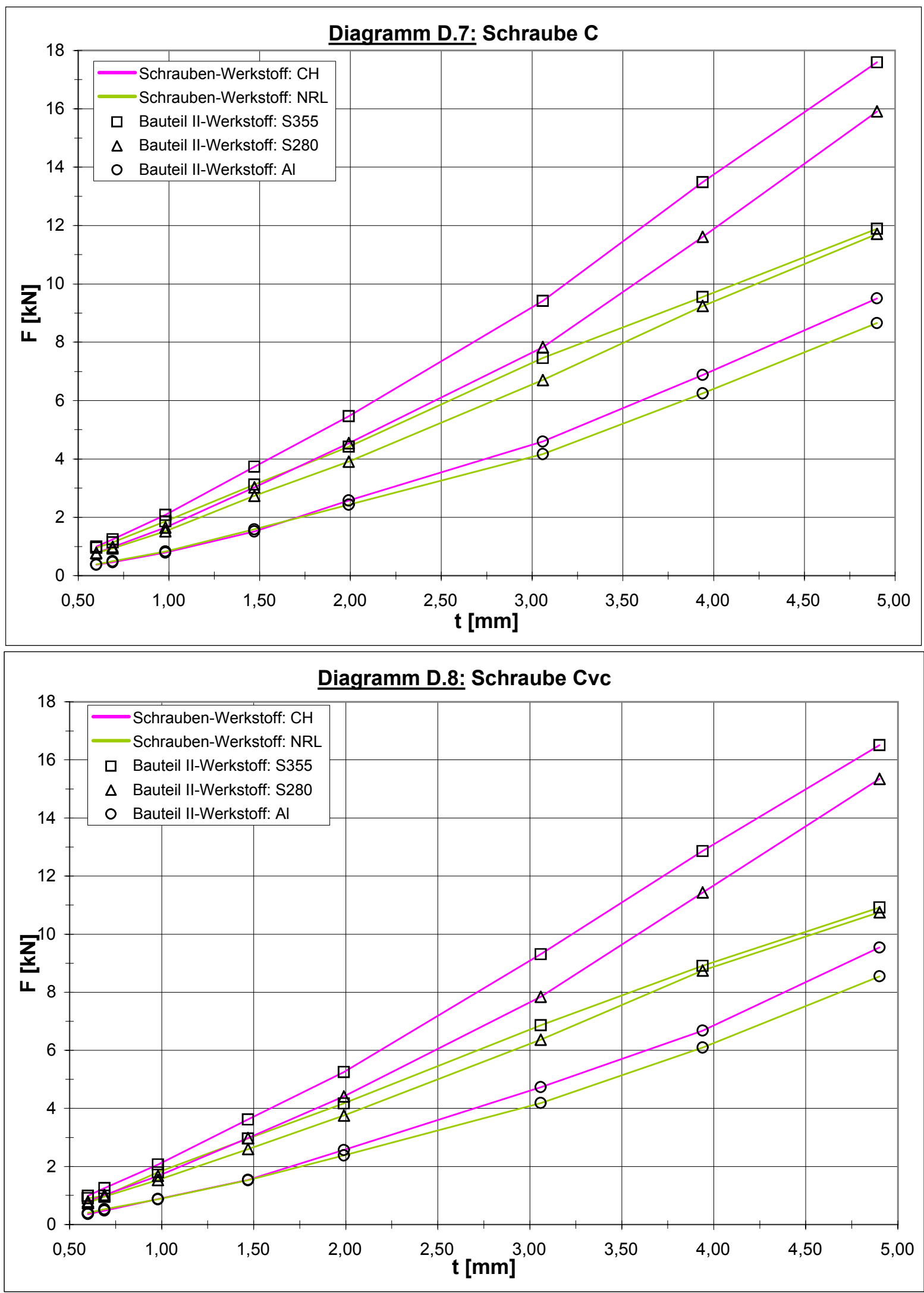
Seite D.5

\section{Diagramm D.9: Schraube A}
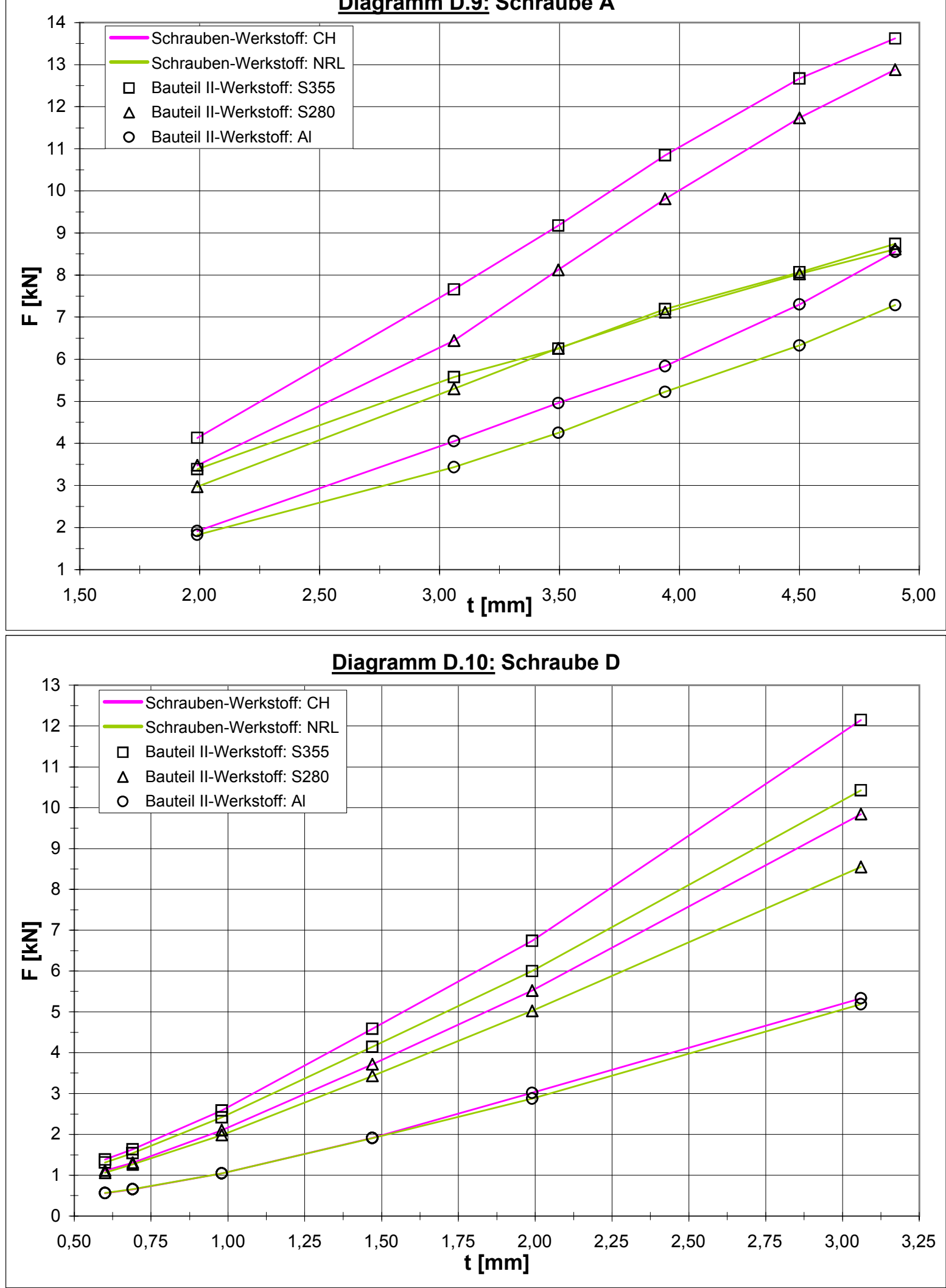
Seite D.6
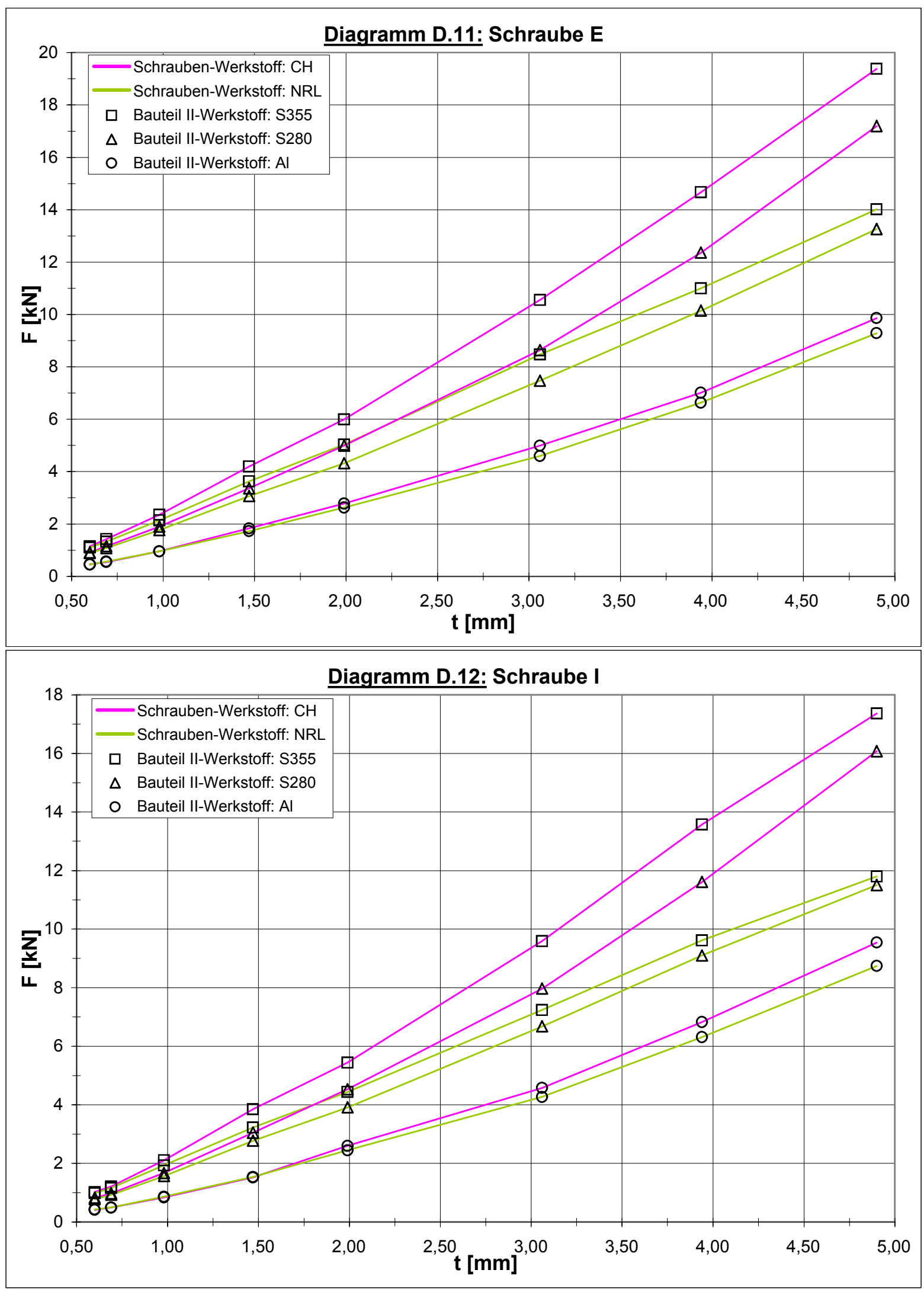
Seite D.7
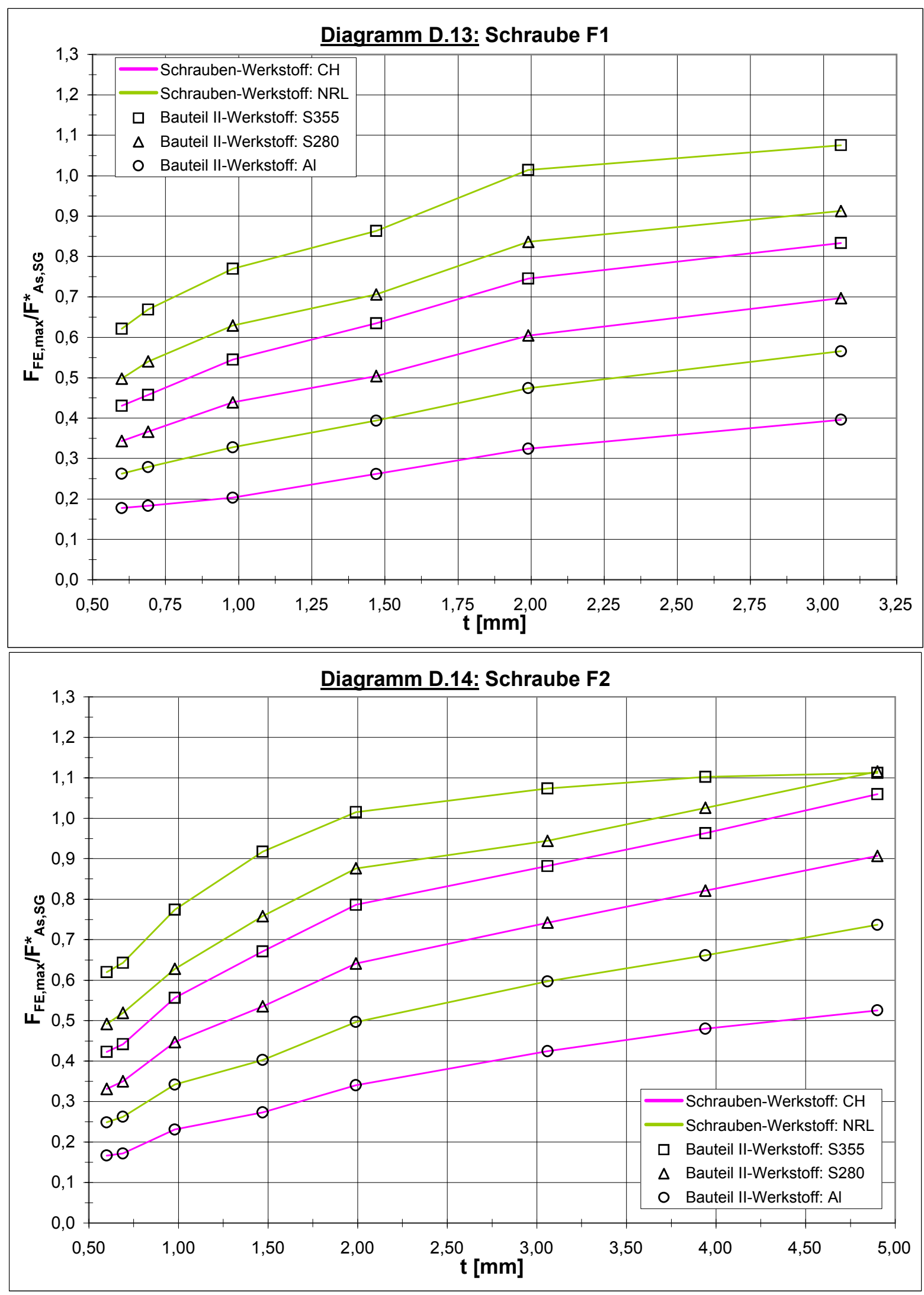
Seite D.8

Diagramm D.15: Schraube B
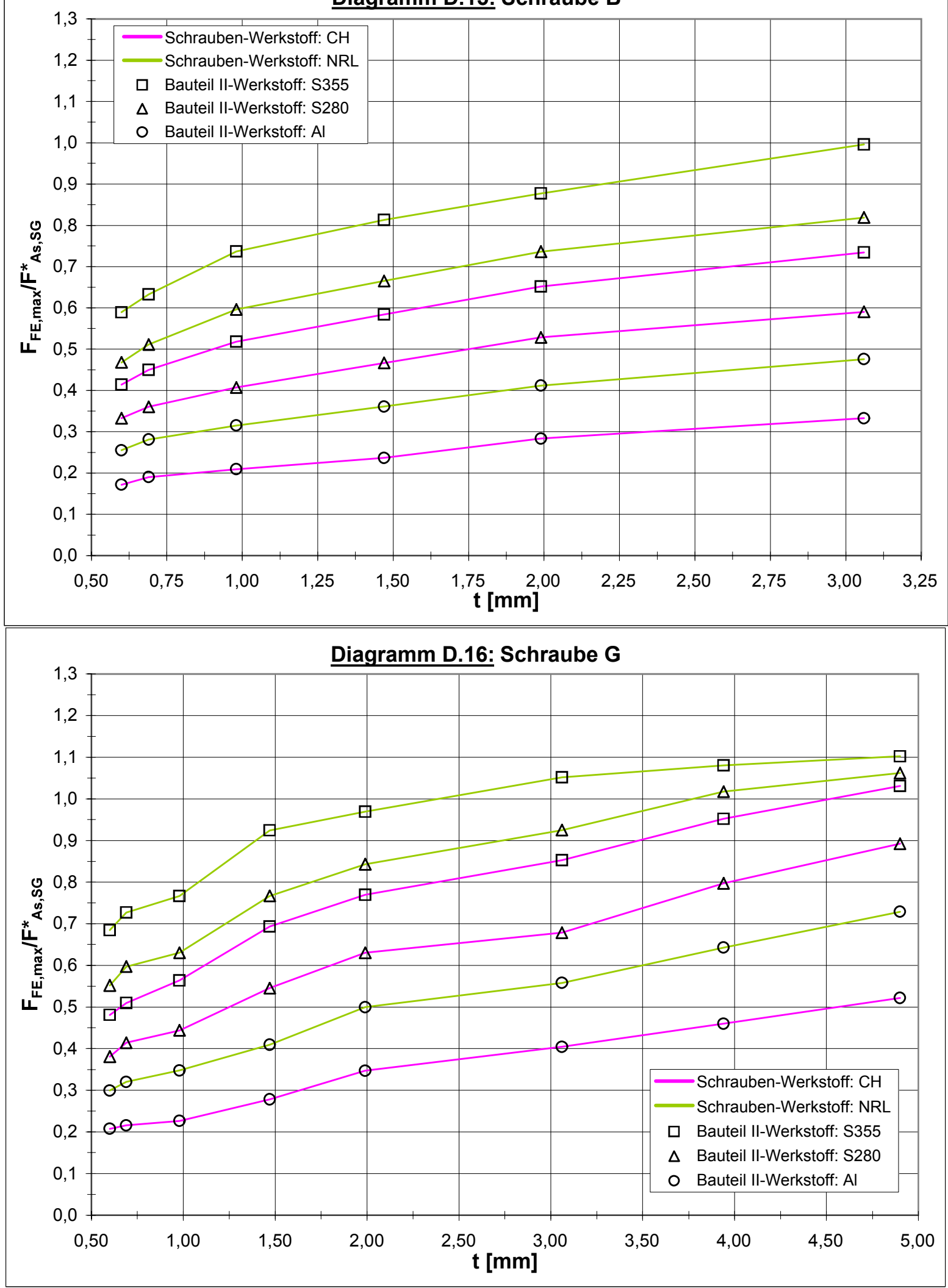
Seite D.9

Diagramm D.17: Schraube J
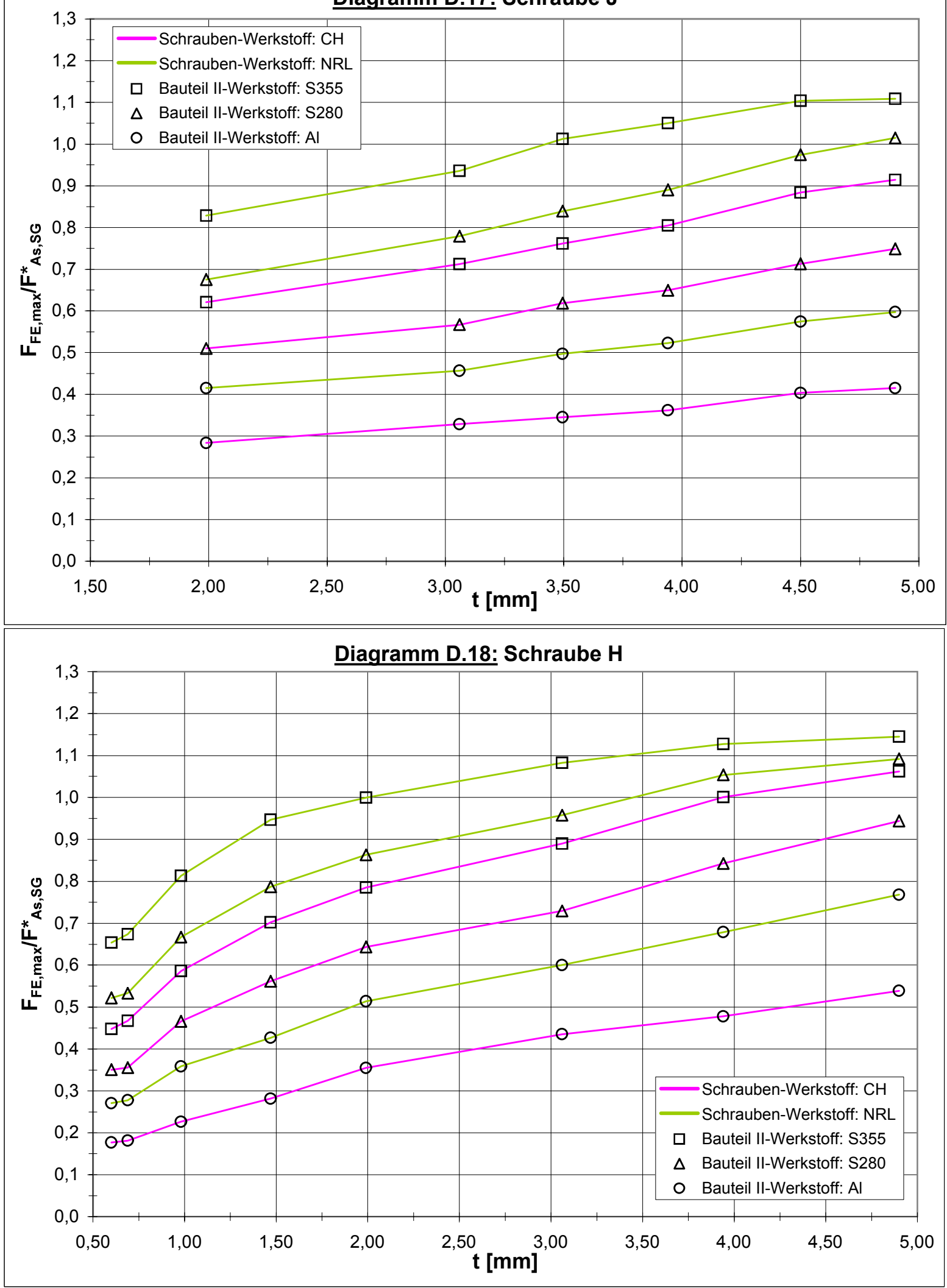
Seite D.10
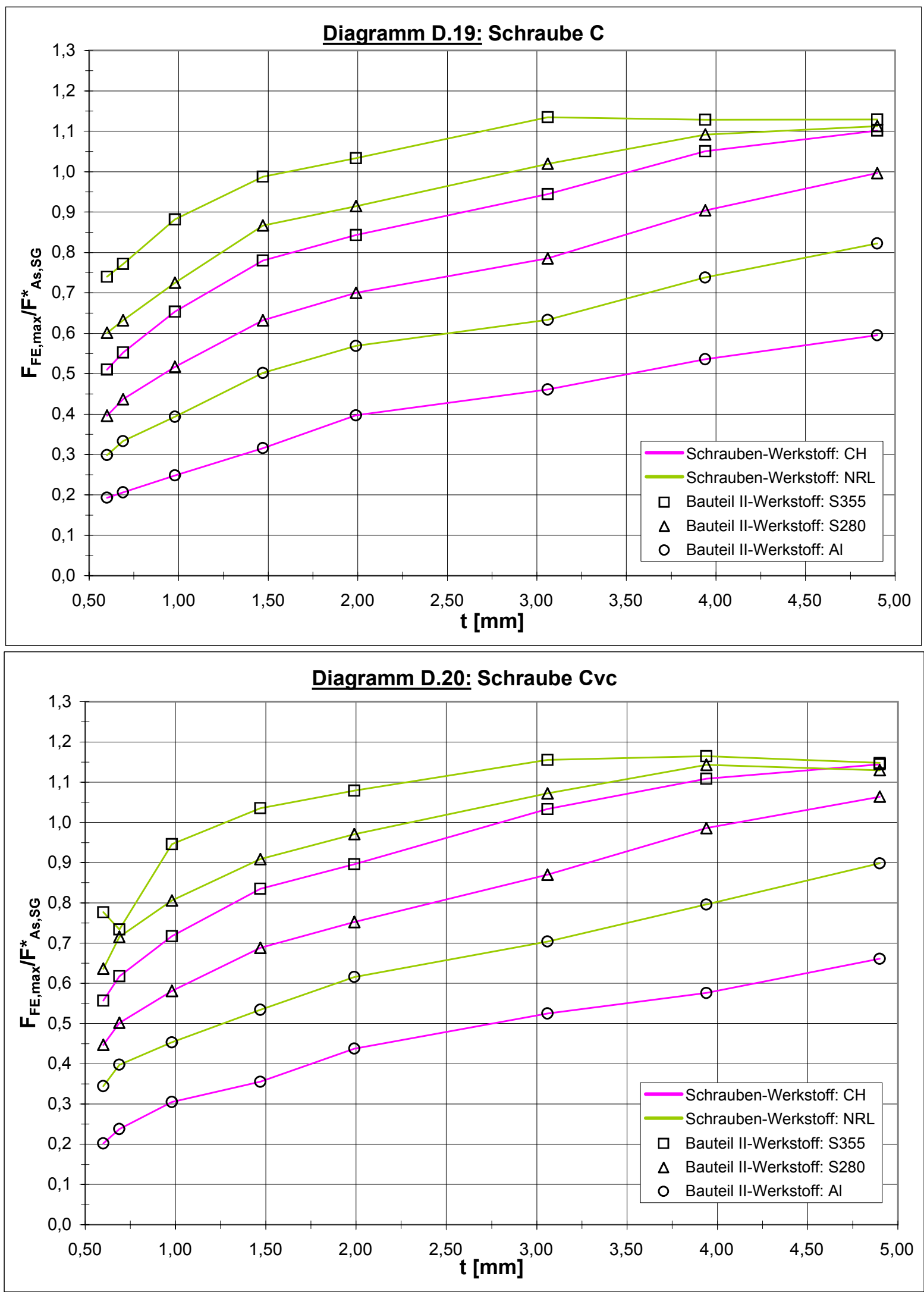
Seite D.11
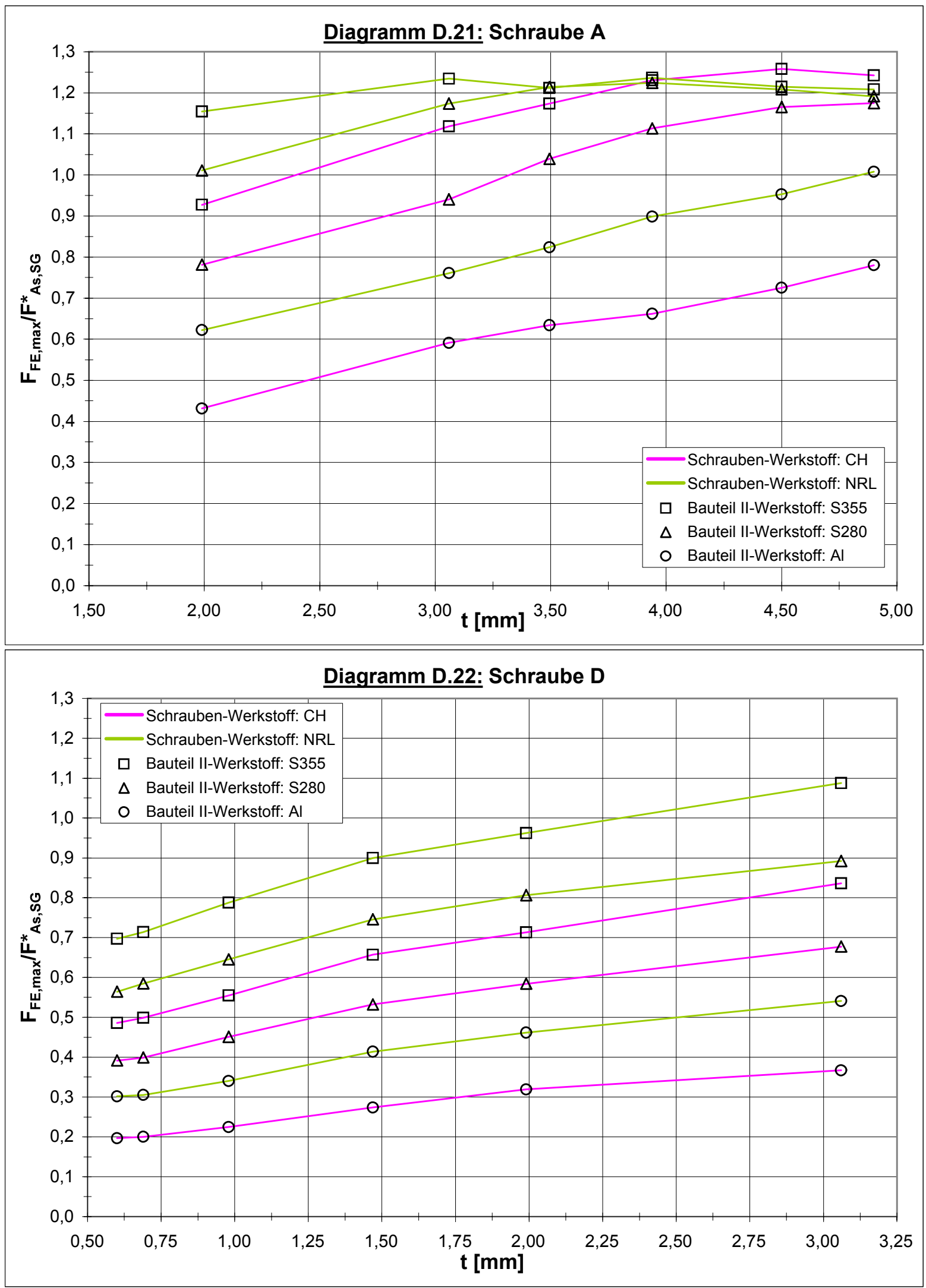

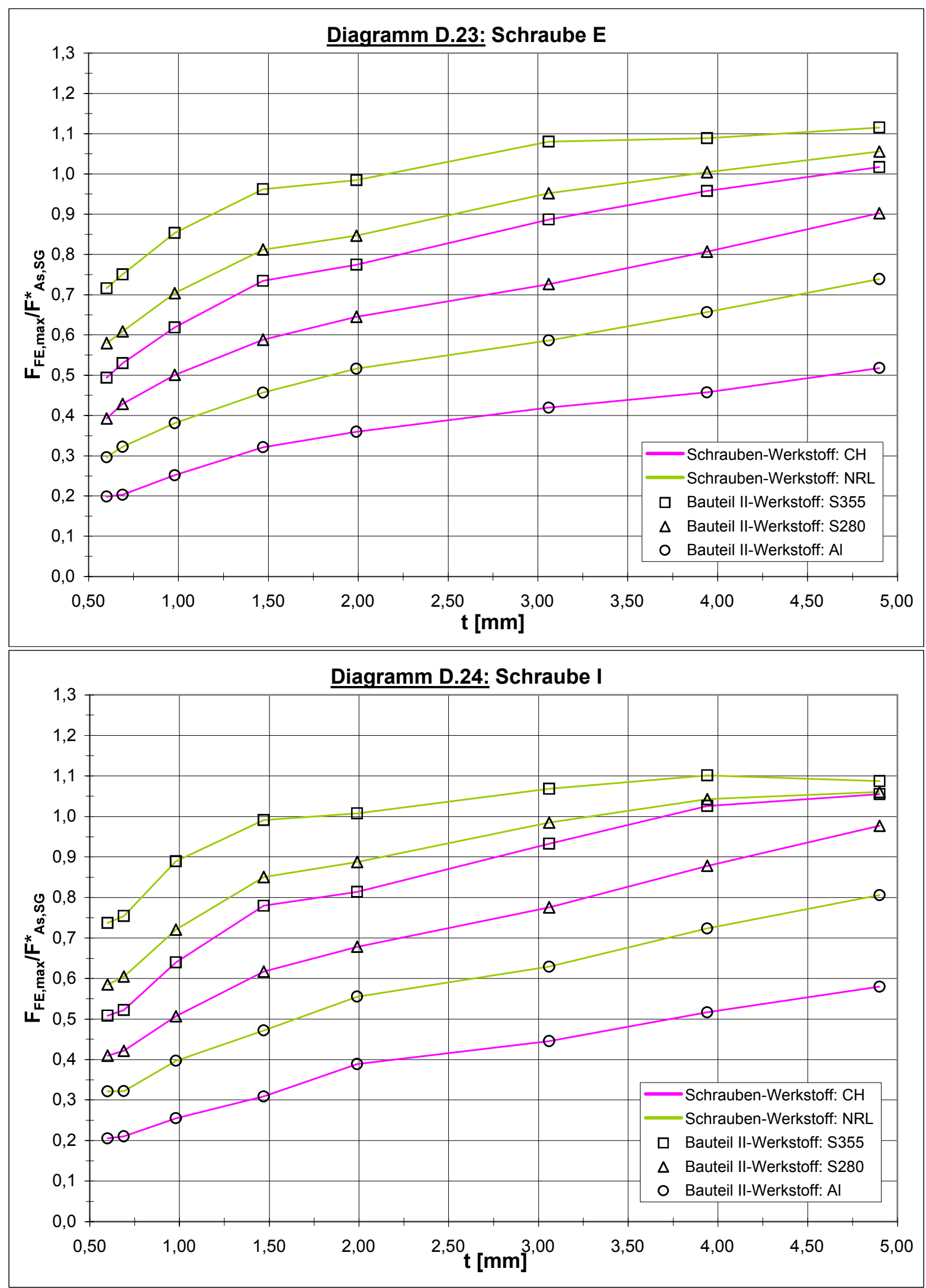


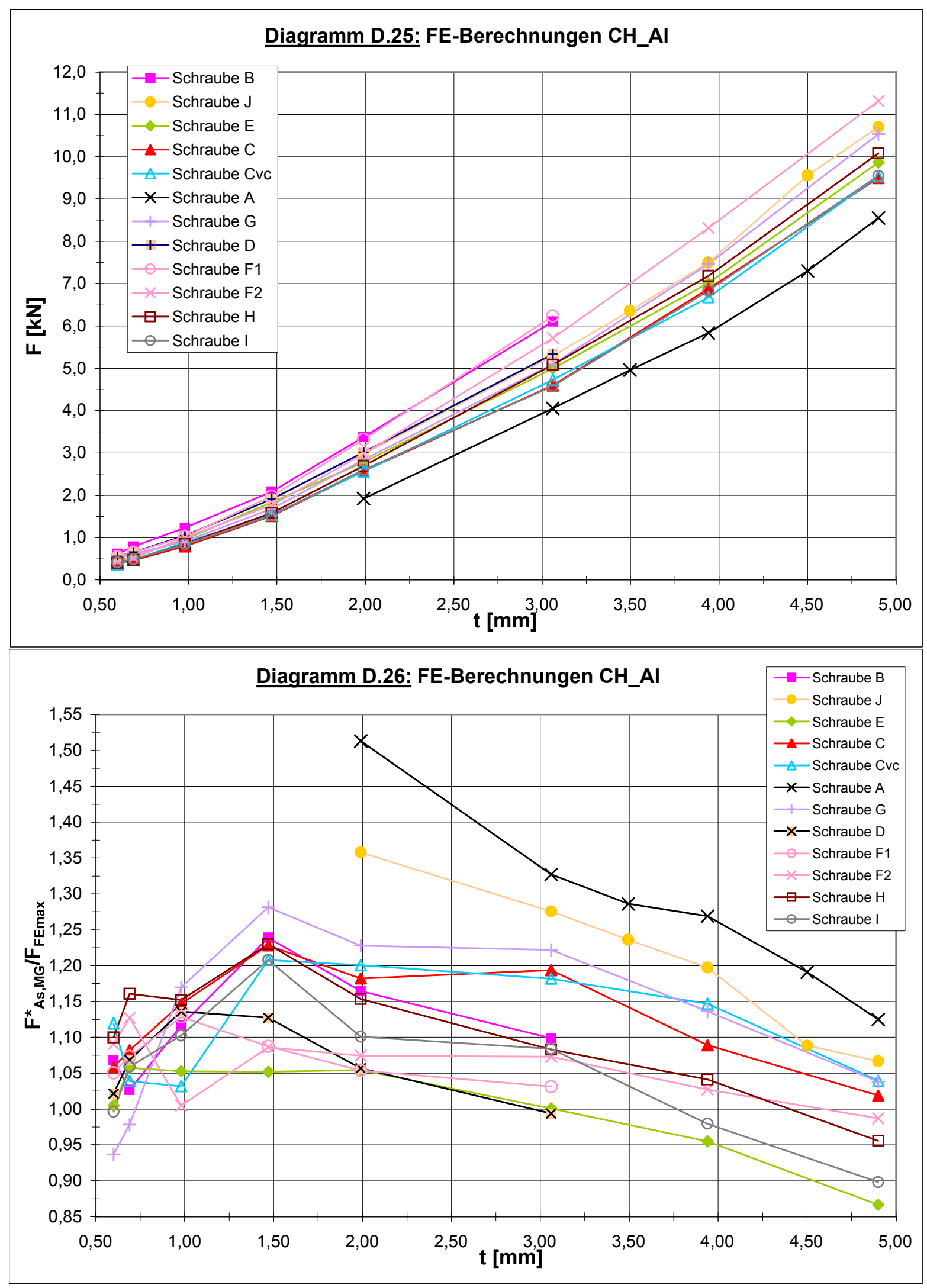


Seite D.14

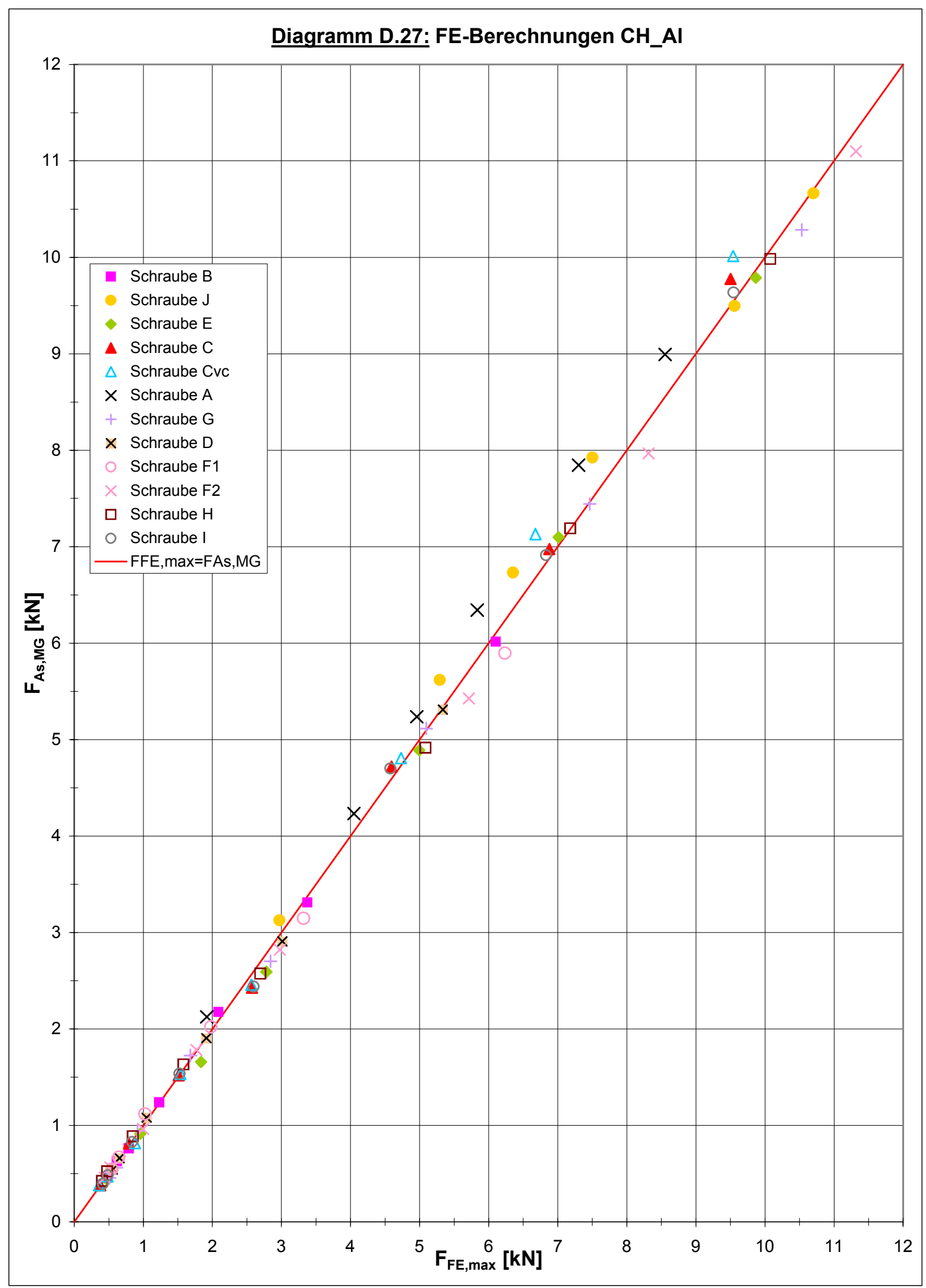


Seite D.15
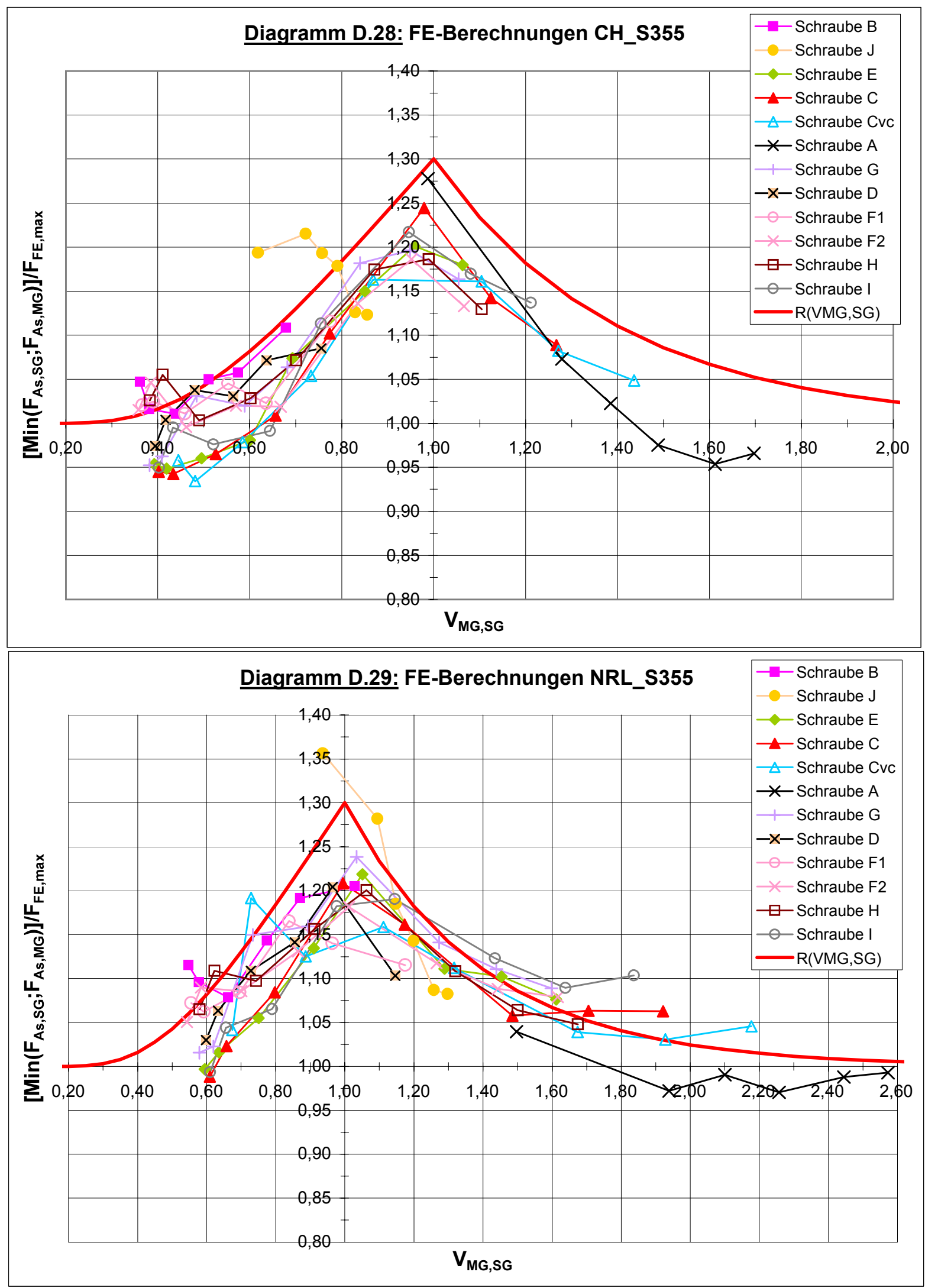
Seite D.16

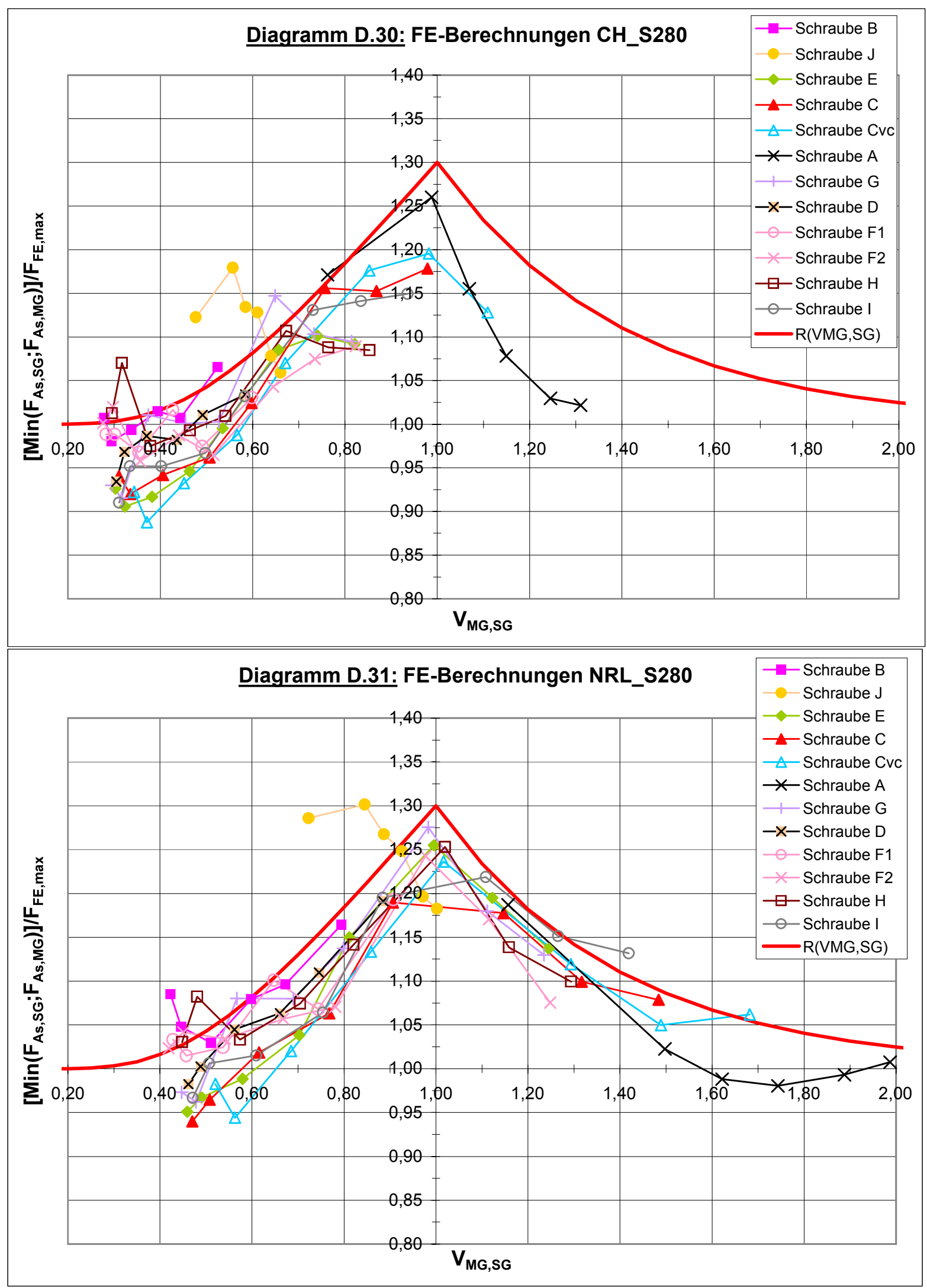




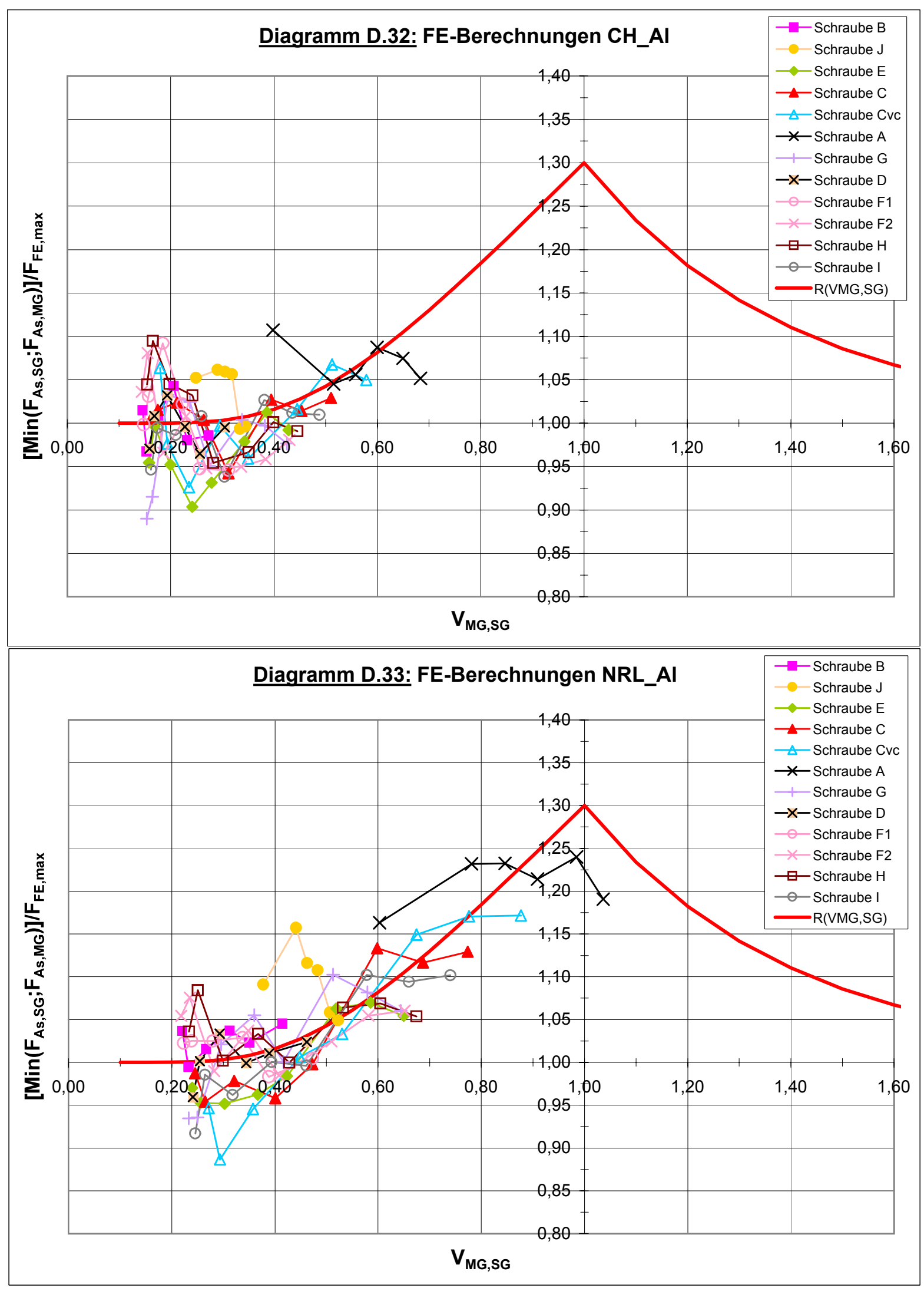


Seite D.18
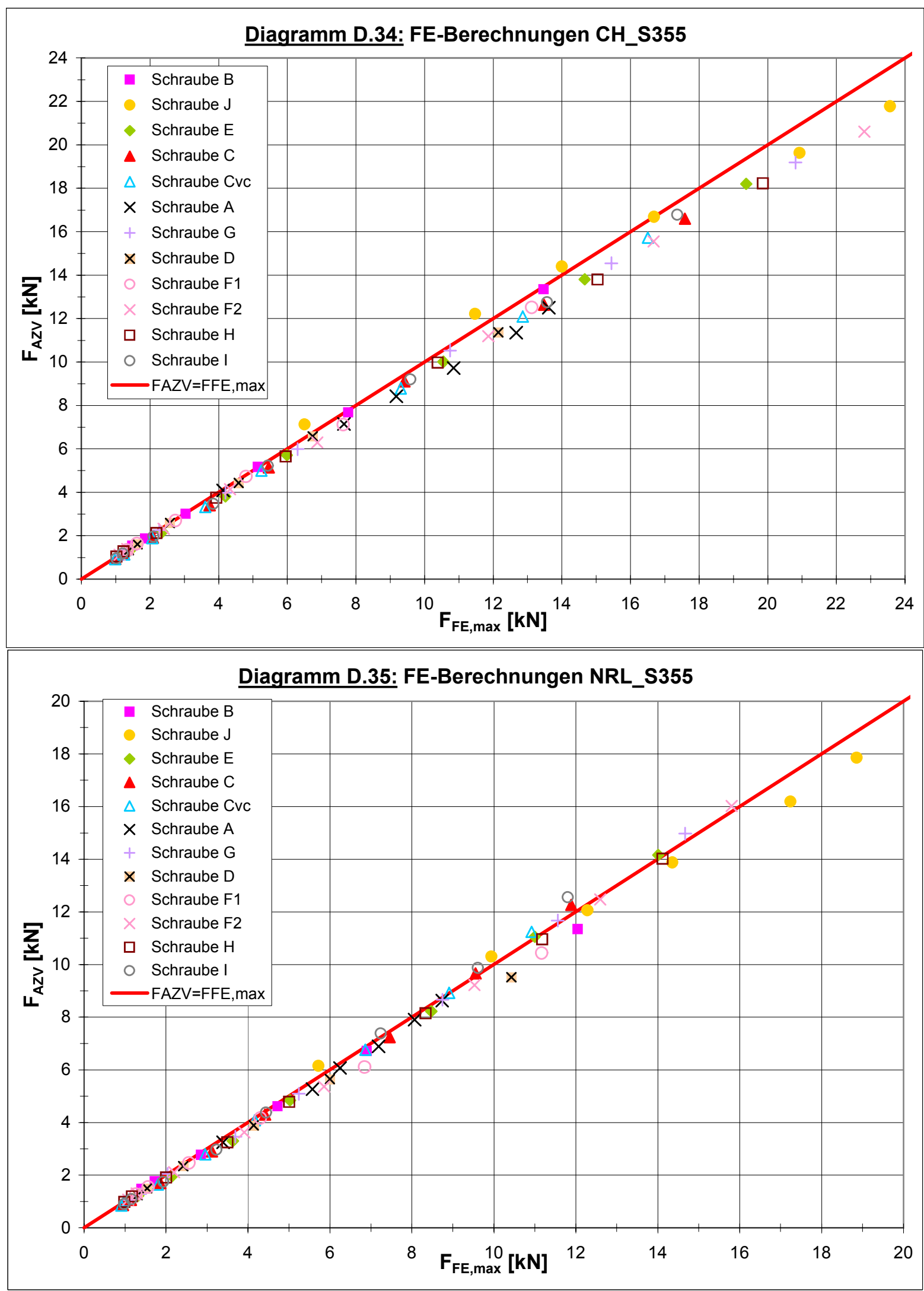
Seite D.19
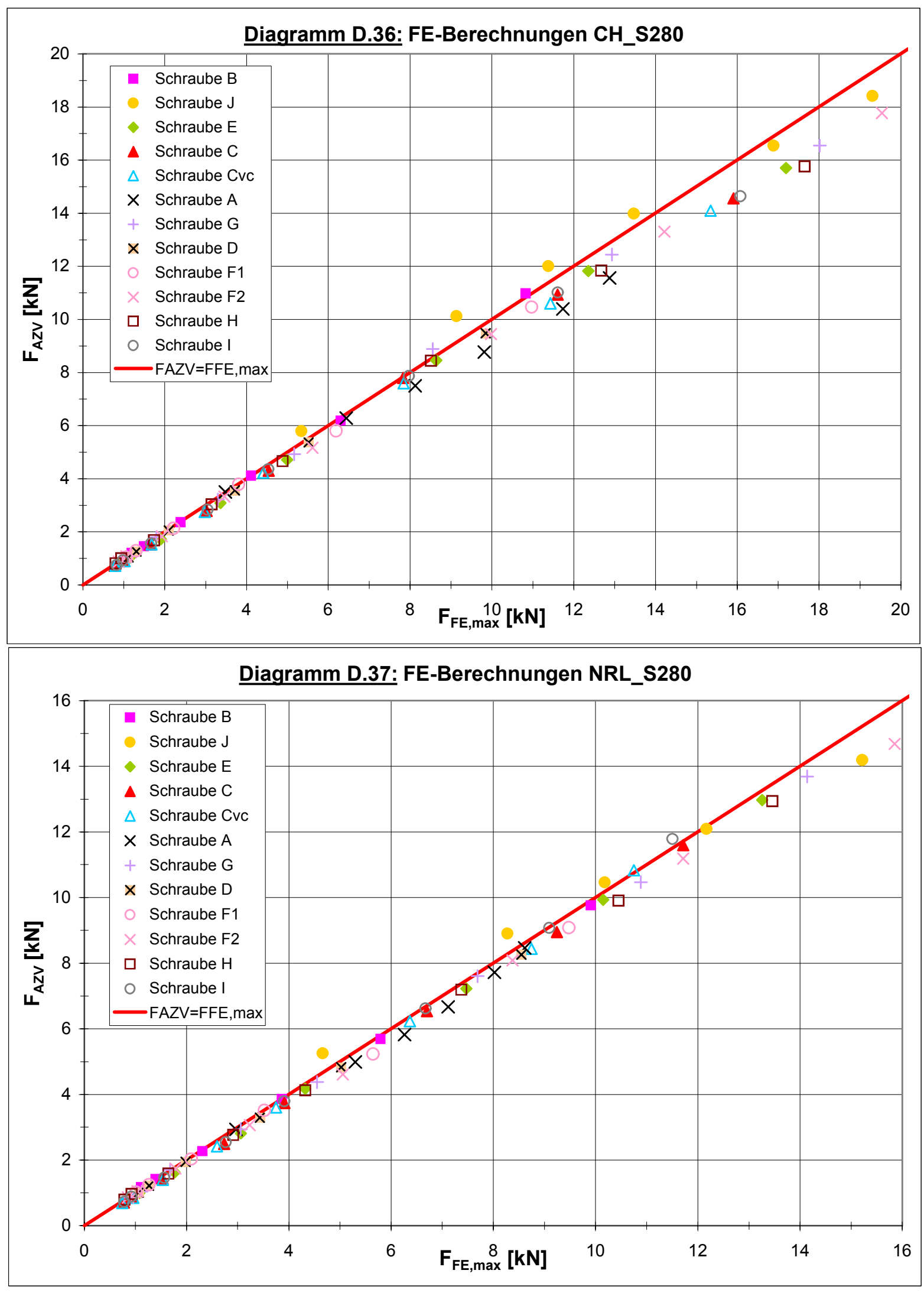
Seite D.20
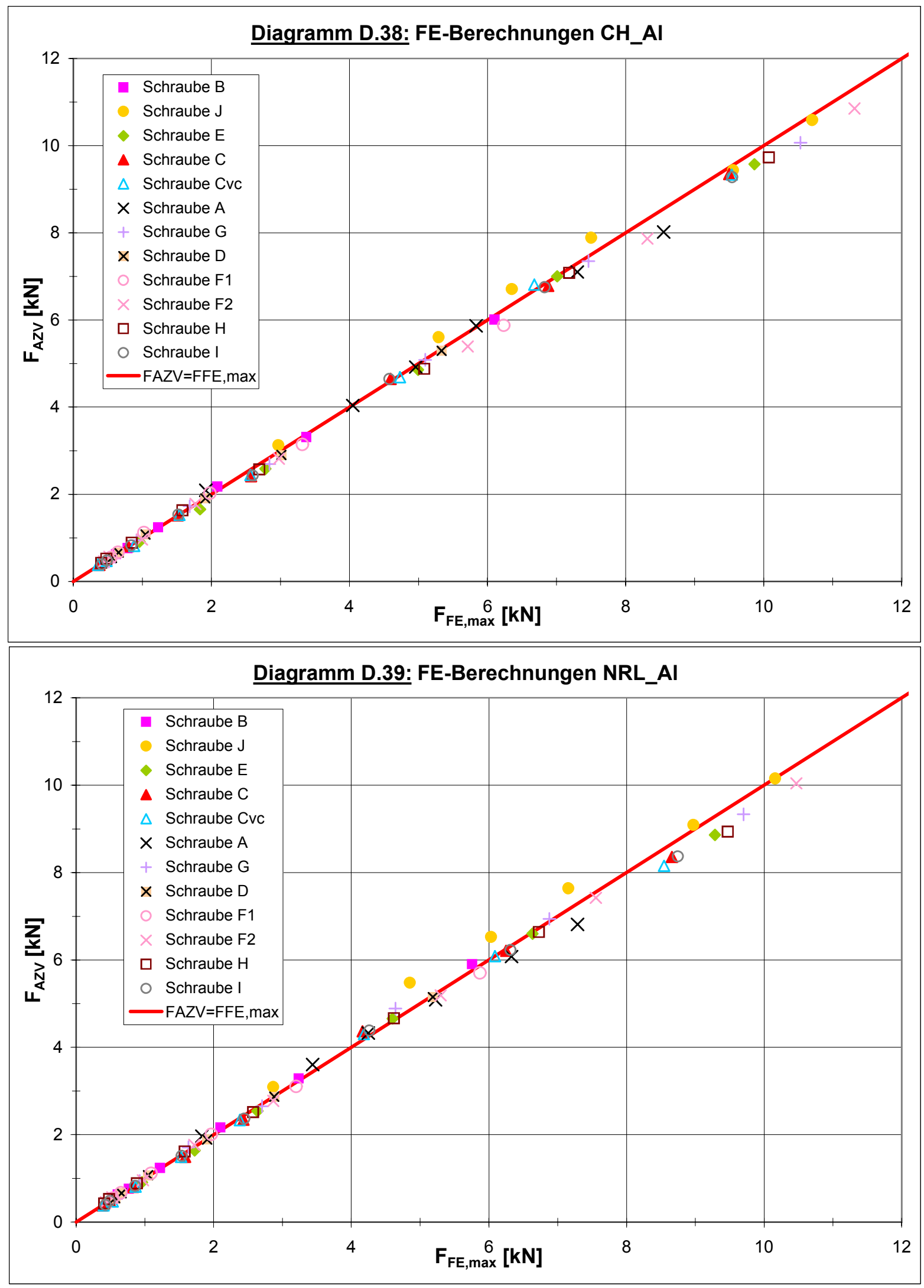